\title{
Theoretical analysis of membrane properties underlying action potential phase-locking in noise-driven cells
}

\author{
PhD Thesis \\ in partial fulfillment of the requirements \\ for the degree "Doctor of Philosophy (PhD)/Dr. rer. nat" \\ in the Neuroscience Program \\ at the Georg August University Göttingen, \\ Faculty of Biology
}

submitted by
Pinar Öz
born in
Ankara, Turkey

Göttingen 2011 
Supervisor: Prof. Dr. Fred Wolf

Co-supervisor: Dr. Andreas Neef

Committee Members: Prof. Dr. Tobias Moser, Prof. Dr. Walter Stühmer Date of submission of the PhD thesis: 14/03/2011

Date of thesis defence: XX/04/2011 


\section{Abstract}

How does the brain work? This very broad and yet, very crucial and interesting question appealed scientists from various disciplines to itself. The fact that the operation of a system of neurons can not be understood without the collaboration of different sciences gave rise to the multi-disciplinary nature of neurosciences. For all the neuroscientists, the most basic question to deal with is how an individual neuron contributes to the rest of the system. Considering the brain as a computational unit, this question becomes as the understanding of the individual neuron as an information processor.

The focus of this thesis project is understanding the mechanisms underlying the initiation of action potentials, which are the units of communication in a neural network, and the relation of these mechanisms with the important characteristics observed in real neurons, such as rapid action potential onsets and threshold variability. These characteristics are crucial and unique, and are in close relation with the linear response properties of neurons: For instance, the first few hundred microseconds of an action potential might reflect important properties of neural response, such as the ability of a neuron to respond efficiently to fast-changing time-varying inputs.

The thesis is organized in three parts. In the first part, a detailed background for the project is given. In this background, information from macro-scale (e.g. the environment that a neuron operates in) to micro-scale (e.g. the molecular organization of the action potential initiation site in the neuron), as well as a brief introduction to the neural transfer functions and neuron models can be found. The information given in this part is quite general and more specific introductions are given for each of the following parts.

In the second part, a multi-compartmental model and the methods of utilizing it to investigate the effect of threshold variability on the somatic spike-triggered statistics are described. The model was constructed modifying Rall's Ball-and-Stick model and its response to noisy inputs were analyzed using spike-triggered average and variance.

In the third part, a recently introduced hypothesis, the existence of cooperative $\mathrm{Na}^{+}$channel gating kinetics in the axon initial segment of a neuron and its contribution to the neural response and action potential waveform, is explained. This hypothesis was investigated on two separate models : a single-compartment model and a multi-compartmental model. Both of these models were conductance-based and the multi-compartmental model was constructed using the real morphological data 
from cortical neurons. The cooperative gating kinetics were implemented in both models, and only in the axon initial segment in the multi-compartmental model.

The results of the models were discussed at the end of each respective part, however, at the end of the thesis, a broader perspective about the results and possible future projects is given. 


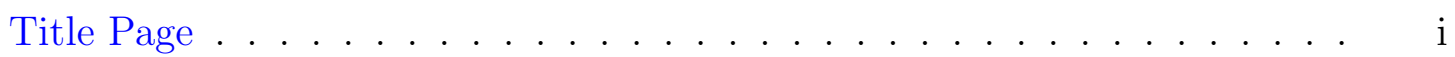

Abstract ............................... ii

Table of Contents . . . . . . . . . . . . . . . . . . $\mathrm{v}$

List of Figures . . . . . . . . . . . . . . . . . . . . ix

List of Tables . . . . . . . . . . . . . . . . . . . . xiii

Acknowledgments . . . . . . . . . . . . . . . . X xv

Dedication . . . . . . . . . . . . . . . . . xviii

I Background $\quad 1$

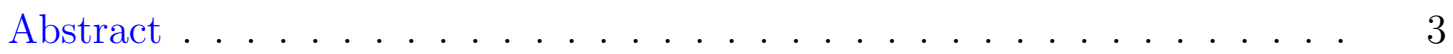

1 The smallest processors of world's most complicated computer 5

1.1 Macro- to micro-scale neuronal computation . . . . . . . . . . 5

1.2 High precision decoding / encoding on a noisy background . . . . . . 6

2 Initiation of the individual electrical signals 9

2.1 Action Potential Generation . . . . . . . . . . . . . . . . 9

2.2 Unique Properties of Initiation Site . . . . . . . . . . . . . . 17

3 Individual Neuronal Output as Linear Response $\quad 29$

3.1 Linear Response Theory . . . . . . . . . . . . . . . . . . 29

3.2 Determinants of Cutoff Frequency . . . . . . . . . . . . . . 30

3.3 Experimental Approach . . . . . . . . . . . . . . . . . . . . . . . . . . . . 32

3.4 Theoretical Approach . . . . . . . . . . . . . . . . . . . 36

4 Imitation of Reality : Neuron Models 37

4.1 Levels of Modeling . . . . . . . . . . . . . . . . . . . 37

4.2 Realistic Approach . . . . . . . . . . . . . . . . . . . . . . . . . . . . . . . . . . . . .

4.3 Simplistic Approach . . . . . . . . . . . . . . . . . . . . 41

II The effect of threshold variability on somatic spike statistics in multi-compartmental models 43

Abstract . . . . . . . . . . . . . . . . . . 45

5 Spike Concept and Threshold $\quad 47$

5.1 Concept of Threshold . . . . . . . . . . . . . . . . . . . . 47

5.2 Spike-Triggered Statistics . . . . . . . . . . . . . . . 48 
6 Multi-compartmental (Ball-and-Stick) Threshold (BST) Model 51

6.1 Model Methods . . . . . . . . . . . . . . . . . 51

6.2 Response Properties in Passive Model . . . . . . . . . . . . . . . . . . 62

6.3 Response Properties in the Active Model . . . . . . . . . . . . . . . . 70

6.4 Dynamic Response in Partially-Active BST Model . . . . . . . . . . . 78

6.5 Summary and Discussion . . . . . . . . . . . . . . . . 85

\section{Numerical Analysis of Cooperative $\mathrm{Na}^{+}$Channel Gat-} ing in the Axon Initial Segment $\quad 87$

Abstract . . . . . . . . . . . . . . . . 89

7 Cooperative gating of voltage-gated ionic channels $\quad 91$

7.1 Experimental Evidence . . . . . . . . . . . . . . . . . 92

7.2 Functional relevance to neural encoding . . . . . . . . . . . . . . . 101

8 Single-Compartment Conductance-Based Cooperative Channel Gating (SC-COOP) Model 103

8.1 Model Methods . . . . . . . . . . . . . . . . . . . . 103

8.2 Activation Kinetics of Cooperatively Gating Sodium Channels . . . . 108

8.3 Action Potential Waveforms . . . . . . . . . . . . . . . . 111

8.4 Impact of Current Statistics on Non-Cooperative Model . . . . . . . . 115

8.5 Impact on Frequency-Current $(\nu$-I) Relationship . . . . . . . . . . . . 120

8.6 Summary and Discussion . . . . . . . . . . . . . . . . . . . . 124

9 Multi-compartmental Cooperative Axon Initial Segment (MCCAIS) $\begin{array}{lr}\text { Model } & 127\end{array}$

9.1 Model Methods . . . . . . . . . . . . . . . . . . . . . . . 128

9.2 Action Potential Waveform in MCCAIS Model . . . . . . . . . . . . 131

9.3 Initiation Site in MCCAIS Model . . . . . . . . . . . . . . 136

9.4 Action Potential Propagation and Conduction Velocity . . . . . . . . 136

9.5 Onset Rapidness of Axonal and Somatic Action Potentials . . . . . . 141

9.6 Linear Response in MCCAIS Model . . . . . . . . . . . . . . . . . . . 141

9.7 Summary and Discussion . . . . . . . . . . . . . . . . 143

IV Summary and Perspective $\quad 147$

$\begin{array}{lll}\text { V Appendix } & 169\end{array}$

$\begin{array}{lr}\text { A Rall Model } & 171\end{array}$

A.1 Boundary Conditions . . . . . . . . . . . . . . . . . . 172 
B Curriculum Vitae 
viii

Contents 


\section{List of Figures}

2.1 Anatomy of an action potential . . . . . . . . . . . 10

2.2 Diversity of APs in central neurons . . . . . . . . . . . . . . 12

2.3 AP energy usage in seven models from vertebrates ans invertebrates . 16

2.4 Energy budget for signaling in the gray matter of the rat brain . . . . 16

2.5 A brief summary of Literature on AP initiation site . . . . . . . 18

2.6 Axon initial segments staining . . . . . . . . . . . . . . . . . . 19

2.7 Simplified stages in AIS development . . . . . . . . . . . 20

2.8 The location of $\mathrm{Na}_{V}$ channel clustering in the axon for neurons with different characteristic frequencies . . . . . . . . . . . . 26

2.9 Intrinsic plasticity on AIS $\ldots \ldots \ldots \ldots \ldots \ldots \ldots$

3.1 Cutoff frequency of the EIF filter in the high-noise regime as a function of the firing rate . . . . . . . . . . . . . . 31

3.2 Cutoff frequency of the EIF filter in the high-noise regime as a function of threshold width, $\Delta_{T} \ldots \ldots \ldots \ldots \ldots$

3.3 Response of neocortical neurons to abrupt changes in input parameters

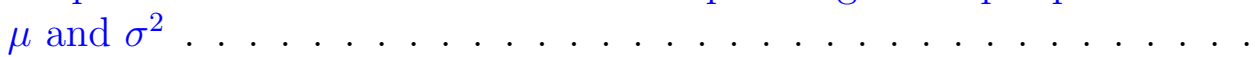

3.4 Modulation depth $\left(r_{1} / r_{0}\right)$ and phase-shift $(\phi)$ of the response to a noisy oscillatory input . . . . . . . . . . . . . . . 35

4.1 Model Comparison . . . . . . . . . . . . . . . . . . . . . . 38

4.2 Examples of five levels of modeling . . . . . . . . . . . . . 39

4.3 A general scheme of a conductance-based (CB) model . . . . . . . . 40

6.1 Passive BST Model . . . . . . . . . . . . . . . . . . . . . . . . . 52

6.2 The ball-and-stick model . . . . . . . . . . . . . . . . . . 52

6.3 The contents of the model in $\mathrm{C}++$ code. . . . . . . . . . . . 58

6.4 3-D representation of subthreshold potential response $\left(\Delta \mathrm{V}=\mathrm{V}-\mathrm{V}_{\text {rest }}\right)$ in passive BST model . . . . . . . . . . . . . . . . . 62

6.5 The current injection and the voltage response in passive BST model 63

6.6 The normalized soma-axon covariances . . . . . . . . . . . . 63

6.7 A typical analysis set for passive BST model . . . . . . . . . . . 64

6.8 Dependence of STA on threshold variability and distance from somatic end in passive BST model under correlated current injection. . . . . . 65

6.9 Dependence of STA on threshold variability and distance from somatic end in passive BST model under OU current injection. . . . . . . . 66

6.10 Dependence of STV on threshold variability and distance from somatic end in passive BST model under correlated current injection . . . . . 66

6.11 Dependence of STA on $\sigma_{I}$ values in passive BST model $\ldots \ldots . .67$

6.12 Dependence of STV on $\sigma_{I}$ values in passive BST model . . . . . . 68 
6.13 Dependence of STA on $\tau_{I}$ values in passive BST model . . . . . . . . 69

6.14 Dependence of STV on $\tau_{I}$ values in passive BST model . . . . . . . . 70

6.15 Dependence of numerically estimated STA and STV on the somatic geometry . . . . . . . . . . . . . . . 71

$6.163-\mathrm{D}$ representation of the action potential propagation in all-active BST model ........................ . . . . 72

6.17 Contour plot representation of a single action potential propagation in all-active BST model . . . . . . . . . . . . . . . . . 73

6.18 Attenuation of $\sigma_{V_{x}}$ by distance from somatic end in all-active BST model 74

6.19 he graphical explanation of partially-active BST model . . . . . . . . 75

6.203 -D representation of the action potential propagation in partiallyactive BST model . . . . . . . . . . . . . . . 76

6.21 Contour plot representation of the action potential propagation from the active compartment in partially-active BST model . . . . . . . . . 77

6.22 The conduction of maximum potential in partially-active BST model $\quad 79$

6.23 Onset latency in partially-active BST model . . . . . . . . . . . . . . 80

6.24 The normalized soma-axon covariance in partially-active BST model . 80

6.25 Attenuation of $\sigma_{V_{x}}$ by distance from active compartment in partiallyactive BST model . . . . . . . . . . . . . . . . . . . . 81

6.26 Dependence of STA (upper figure) and STV (lower figure) on threshold variability and distance from somatic end in partially-active BST model 82

6.27 Typical examples of Raster Plot (uppermost), PSTH (middle) and $\nu(\mathrm{t})$-t plot(lowermost) . . . . . . . . . . . . . . . 83

6.28 Gain modulation under sinusoidally modulated input . . . . . . . . . 84

7.1 Coupled gating of KcsA channels in the LOP pattern . . . . . . . . . 93

7.2 Coupled gating of KcsA channels in HOP patterns . . . . . . . . . . . 94

7.3 Reconstructed giant liposome under confocal microscopy . . . . . . . 95

7.4 Ryanodine receptor clustering observed by thin-layer electron microscopy 97

7.5 Amplitude analysis of LPC-modified $\mathrm{Na}^{+}$channel current and examples of channel activities from an inside-out patch at a membrane potential $\mathrm{V}_{\text {mem }}=-70 \mathrm{mV} \ldots \ldots$. . . . . . . . . . . . . 98

7.6 Amplitude analysis of LPC-modified $\mathrm{Na}^{+}$channel current and examples of channel activities from an inside-out patch at a membrane potential $\mathrm{V}_{\text {mem }}=-180 \mathrm{mV} \ldots \ldots . \ldots . \ldots . \ldots 99$

8.1 The spherical single compartment model . . . . . . . . . . . . . . . . 104

8.2 The activation kinetics of $\mathrm{Na}^{+}$channels in cooperative and non-cooperative model ........................ 109

8.3 The collective activation curves for $\mathrm{x}=1$ (upper figure) and $\mathrm{x}=3$ (lower figure) . . . . . . . . . . . . . . . . . 110 
8.4 The upper figure is the phase-plot representation of the action potential in the lower figure . . . . . . . . . . . . . . . . . . . . 112

8.5 Phase plots for different coupling strengths and coupling percentages $(K J=20-200 \mathrm{mV}) \ldots \ldots \ldots 113$

8.6 Phase plots for different coupling strengths and coupling percentages $(K J=600-800 \mathrm{mV}) \ldots \ldots \ldots \ldots 114$

8.7 The change in onset rapidness $(\Gamma)$ with increasing coupling strength . 115

8.8 Effect of $\sigma_{I}$ on model firing rate . . . . . . . . . . . . . . . . . . 116

8.9 Effect of $\tau_{I}$ on model firing rate . . . . . . . . . . . . . . . 117

8.10 Effect of $\sigma_{I}$ on action potential peak values . . . . . . . . . . . . . . 117

8.11 Effect of $\tau_{I}$ on model action potential peak values . . . . . . . . . . . 118

8.12 Effect of $\sigma_{I}$ on subthreshold membrane potential deviations . . . . . . 118

8.13 Effect of $\tau_{I}$ on subthreshold membrane potential deviations . . . . . . 119

8.14 The comparison of $\nu$-I relationship in SC-COOP and original WB models 121

8.15 The $\nu$-I curves for $K J=800 \mathrm{mV}$ under constant current injection . . 122

8.16 The $\nu$-I curves for $p=10 \%$ under constant current injection. . . . . . 122

8.17 The $\nu$-I curves for $K J=600 \mathrm{mV}$ under noisy current injection . . . . 123

8.18 The phase diagram for $\nu$-I curves under noisy current injection . . . . 123

9.1 The geometry of Multi-Compartmental Cooperative AIS (MCCAIS) model . . . . . . . . . . . . . . . . . . . . 130

9.2 AP waveforms in MCCAIS Model $\left(g_{\text {soma }}=g_{\text {dend }}\right) \ldots \ldots$. . . . . . . 132

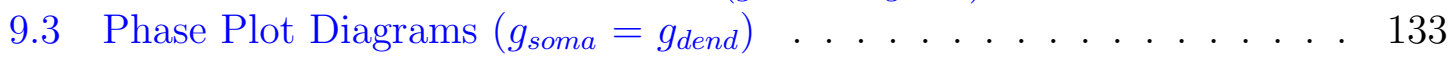

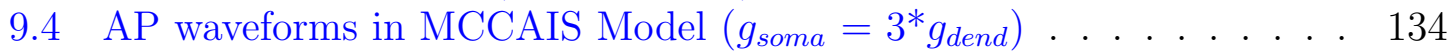

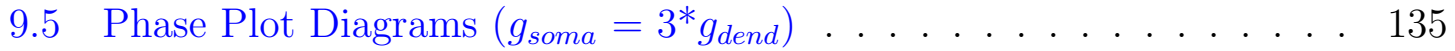

9.6 Action potential initiation and propagation . . . . . . . . . . . . 137

9.7 Action potential initiation and propagation in 3D . . . . . . . . . 138

9.8 AP propagation in MCCAIS model for non-cooperative and cooperative model . . . . . . . . . . . . . . . . . . . . . . . . . . . . . . . . 138

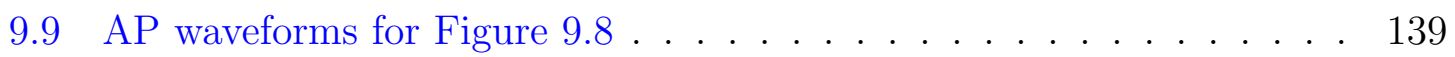

9.10 AP propagation in MCCAIS model for varying channel distributions in non-cooperative MCCAIS model . . . . . . . . . . . . . . . . . . . 140

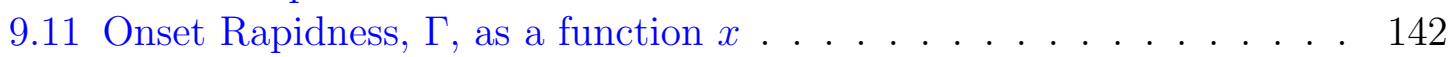

9.12 The location of maximum $\Gamma$ as a function $K J$. . . . . . . . . . . . 143

9.13 The Frequency-Current (f-I) relationship for non-cooperative MCCAIS model .......................... 144 


\section{List of Tables}

4.1 A general overview for integrate-and-fire models in the literature . . . 42

6.1 Wang-Buzsaki model parameters . . . . . . . . . . . . . . 61

8.1 The voltage dependence of $\mathrm{Na}^{+}$and $\mathrm{K}^{+}$type channels in Wang-Buzsaki model ....................... 105

9.1 The parameters of Multi-Compartmental Cooperative AIS (MCCAIS) Model . . . . . . . . . . . . . . . . . . . . 129

9.2 The channel density distribution for MCCAIS . . . . . . . . . . . . . 131

9.3 Axonal conduction velocities with respect to channel densities in AIS and NR . . . . . . . . . . . . . . . . . . . . . . . . . 139

9.4 Dendritic conduction velocities with respect to channel densities in AIS

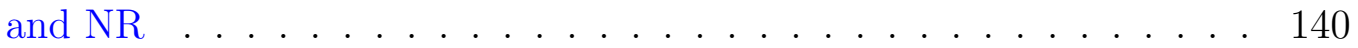




\section{Acknowledgments}

First of all, I would like to thank my advisor Prof. Dr. Fred Wolf for his excellent guidance through my $\mathrm{PhD}$. Without his patient teaching and continuous trust, this work would not have been successful. Secondly, I would like to thank my co-advisor Dr. Andreas Neef and Prof. Dr. Tobias Moser for patiently guiding me through my work by sharing their experiences with me and for countless hours of fruitful discussions. I also would like to thank Prof. Dr. Walter Stühmer for being in my thesis committee and for sharing valuable ideas. This work has been done in collaboration with Dr. Min Huang and Dr. Wei Wei, so I am very thankful for their efforts and friendly supports, too. Additionally, I would like to thank Mike Gutnick, Ilya Fleidervish and Maxim Volgushev for valuable comments and suggestions.

I especially thank to Prof. Michael Hörner and Sandra Drube in IMPRS Neuroscience Program for taking thoughtful care of us international students, supporting us in our adaptation period for a completely new environment and also helping in every aspect related to the administrative work.

I would like to thank all my colleagues and friends for their direct and/or indirect input to my work and a having great time together. Many thanks go to Georg Martius and Wei Wei, especially for the warm environment of our working space; Michael Monteforte for his help on programming, Georg Martius and Yorck Beensen for all the technical support; my colleagues Ghazaleh Afshar and Dominika Lyzwa for the enthusiastic discussions and for our honest, wonderful friendship; Eren Erdal Aksoy for his patient help on LateX; my wonderful friends Esra Demircioglu, Tuba Pekkirbizli, Buket Ozkaya, Cansu Topal, Eren Erdal Aksoy and Mehmet Gultas for every moment and every bright idea that we shared; my mother Ayse Arslan for being an extra pair of eyes and hands at the time that I needed most and all the friends who supported me through my journey in Göttingen.

A special thanks goes to my mother and my father: Without their limitless support, light, love and wisdom, I would not be who I am and I would not have achieved all that in my life what I have now.

Thank you all very much! 
Herewith I declare that I prepared the PhD Thesis

"Theoretical and experimental analysis of membrane properties underlying action potential phase-locking in noise-driven cells"

on my own with no other sources and aids than quoted.

Göttingen, March 14th, 2011

Pinar $\ddot{\mathrm{O} z}$ 
Dedicated to my mother Ayse, and my father Adil. 


\section{Part I}

\section{Background}





\section{Abstract}

The ionic channels are the main elements of the excitable membranes. Most of the ionic channels are not always in the open state but usually depend on the existence of a certain stimulant (e.g. voltage difference, chemical binding, mechanical stretches and so on) to get activated. An important fraction of these channels, the voltage-gated ionic channels, sense the voltage changes on the excitable membranes and open or close in response. Their activity further changes the membrane potential and the electrical signal is amplified or degraded. Therefore, the voltage-gated ionic channels are extremely important for spreading a local excitation on the whole membrane surface.

The governing principles of electrical invasion on the neuronal membrane is, therefore, closely related to the principles of voltage-gated ion channel gating. It is only possible with the accurate activity of these channels that an impulse from the presynaptic neuron can be translated into an electrical message, which is then carried along the dendritic, somatic and axonal membranes to the synapse and then to the postsynaptic neuron. A specific presynaptic input is delivered to the cortical neuron in a noisy background, due to the simultaneous bombardment of excitatory and inhibitory inputs. Therefore, the accurate recognition of a certain input in the noisy background and the precise timing of the response becomes crucial.

The capability of a neuron to elucidate precisely-timed action potentials (APs) in response to the very small fluctuations in the noisy input can be indirectly interpreted using the steepness of the AP onset (Naundorf et al. [132]; Köndgen et al. [100]; Fourcaud-Trocme et al. [63]). In this study, we utilized this indirect tool to explore the dynamic response properties of conductance-based neuron models, in which stochastically non-independent sodium channel gating kinetics were implemented. 


\section{The smallest processors of world's most complicated computer}

Cognitive functions of the brain, such as in the visual or in the auditory system require fast, precise and reliable processing of the information about the surrounding environment. For instance, the recognition of a natural image by the human visual system requires less than $150 \mathrm{~ms}$ (Thorpe et al. [177]; Hopfield [85]). It was also shown that, for instance, the accuracy and the reliability of auditory responses in locust are highly sensitive to the stimulus statistics (Machens and Zador [115],Machens et al. [114]). How does the neural system achieve this fast and sensitive response? Does the fine-tuning of information processing occur in population level or in single-neuron level? In the next sections, I will summarize some of the previous studies on neuronal computation and its fine-tuning in noisy environment.

\subsection{Macro- to micro-scale neuronal computation}

The individual neurons, e.g. of the visual cortex, are silent most of the time and have a spontaneous firing rate of only 1-2 Hz (Greenberg et al. [70]; Destexhe and Parè [49]). Then, how can the brain respond to fast-changing stimuli? The answer lies in the population coding. The coordinated activity of a group of neurons allows to track down and phase-lock to the fast-changing stimuli with temporal frequencies much higher than the firing rate of an individual neuron. The population coding has a number of advantages. For instance, the uncertainty due to neuronal variability is reduced and a number of different stimulus attributes can be simultaneously represented (Rieke et al. [152]).

The population activity can be recorded as local field potentials. The recordings from cerebral cortex revealed that the population firing rate is much higher than the firing rate of individual neurons (Buzsaki and Draguhn [29]). When instantaneous 
firing rates of individual neurons are averaged over a neuronal ensemble, the fast oscillation patterns could be observed in the response.

Although it is the collective behavior that produces the required phase-locking response, it is crucial to understand the action potential (AP) generating mechanisms underlying the individual responses to understand the collective response, and, when a realistic explanation is aimed, time-varying inputs must be used to test the temporal precision of the individual neuronal output.

\subsection{High precision decoding / encoding on a noisy background}

Understanding the neural code would necessarily mean making sense out of massive information flow among the brain and the sensory organs in the form of arrays of spike trains. Any meaningful explanation would require some boundaries on the definition of stimulus. In many psychophysical discrimination experiments (Green and Swets [69]), the stimulus defines a world of two choices. This kind of binary decision problems are useful in investigating the reliability of neurons; however, it is not enough or, as in many cases, realistic. The natural stimulus, as it would be expected, is anything but binary or stationary (Rieke et al. [152]). Under natural conditions, the properties of the stimulus in the next brief time window can not be fully predicted. Therefore, the natural stimulus could be best represented as picked from an infinite set of alternatives that are not equally probable (Rieke et al. [152]).

Even though having this stochastic characteristics, the natural stimulus also contains an underlying structure or regularity.

To sum up, the natural signals will be randomly presented to the organism, although they will have origins in deterministic processes.

The phenomena explained above is one of the reasons for using random but correlated time-dependent signals in in vitro and theoretical studies. Other reasons come from the background activity in the brain and intrinsic noise of the neuron due to ionic channels.

Neurons in vivo are continuously bombarded by excitatory and inhibitory synaptic inputs, which strongly affect their response properties (Silberberg et al. [166]; Arsiero et al. [10]; Brunel et al. [23]; Chance et al. [36]; Fourcaud-Trocme and Brunel [62]; Destexhe and Parè [49]; Hô and Destexhe [81]; Anderson et al. [5]). This effect might be due to, e.g., the increase in the overall conductance (Borg-Graham et al. [19]; Destexhe and Parè [49]) or in the neuronal response variability (Softky and Koch [168]; 
Holt et al. [84]; Stevens and Zador [171]; Shadlen and Newsome [162]). Therefore, this background activity should be taken as a constant source of noise in the input that neuron receives. The resulting barrage of background synaptic input increases the total conductance of the neuron by 2-3 times its resting value (Borg-Graham et al. [19]; Destexhe and Parè [49]) and the fluctuations in the input induce fluctuations in membrane potential that have an amplitude of a few $\mathrm{mVs}$ (Anderson et al. [5]). The spike time pattern may also change as a response to the driving current (Chance et al. [36]).

The in vitro and theoretical studies will lack the contribution of background noisy input. To compensate, artificially generated noisy currents are often used.

It was shown that the statistics of noise affect the precision, reliability and also the amplitude of the response (Svirskis [174]; Arsiero et al. [10]; Brunel et al. [23]; Chance et al. [36]; Fourcaud-Trocme and Brunel [62]; Wiesenfeld and Moss [186]; Herrmann and Gerstner [78]; Svirskis and Rinzel [175]; Allen and Stevens [3]). For instance, stronger noise will force the membrane potential to fluctuate near the threshold quite often. It is obvious that this will increase the probability of threshold crossing very sharply at the arrival of a signal.

To sum up, neurons actually need the noisy background activity to generate fast and precise response to the incoming stimulus. The noise can help detecting rapid and/or small subthreshold signals. Additionally, the fluctuations in the membrane potential keeps the neuron ready for the fast response to incoming stimuli. However, the statistics of the input noise affect the response properties both in favor of and against the accurate and reliable response, as it also presents boundaries for the ability of a neuron to respond. As will be explained in chapter 3, the frequency of the fluctuations in the input is probably the most important constraint on linear neuronal response. 
8 Chapter 1: The smallest processors of world's most complicated computer 


\section{2 \\ Initiation of the individual electrical signals}

Considering the information flow through the elaborately wired circuits of the brain, the APs are the units of information. The electric signal is produced as APs in the individual neurons and conveyed as information with their timings, or spike trains. Obviously, to understand the neural code and the information it contains, it is essential to understand the generation of individual signals.

\subsection{Action Potential Generation}

A neuron elicits an AP when its membrane potential is depolarized above the threshold potential, which is the minimum for the activation and opening of voltagegated $\mathrm{Na}^{+}$channels (Figure 2.1). This supra-threshold depolarization triggers an avalanche-like process : opening of $\mathrm{Na}^{+}$channels further depolarizes the membrane and thus, triggers the opening of more $\mathrm{Na}^{+}$channels and also different types of voltage-dependent ionic channels. In some cases, the term spike can be used instead of the AP, however, it especially means the portion of AP with the most rapidly changing voltage. It is also very commonly used to mean solely the signal representation of an AP and even in some cases, the full AP waveform may not be needed to define a spike (see section 5.1).

\subsubsection{The Waveform}

The shape of the AP varies considerably for different types of neurons (Figure 2.2). The invertebrate and vertebrate AP are proven to have especially different at their onset (Volgushev et al. [182]; Sengupta et al. [161]). The mammalian brain cells still exhibits a broad variety of AP shapes. Although the firing behavior of neurons include many different patterns and frequencies with little or no obvious relation with AP shape, there are still cases, such as that a neuron eliciting "narrow" APs usually exhibit an ability to fire at high frequencies with a little decrease in the frequency for prolonged stimulations (Connors and Gutnick [42]; McCormick et al. [122]). The 


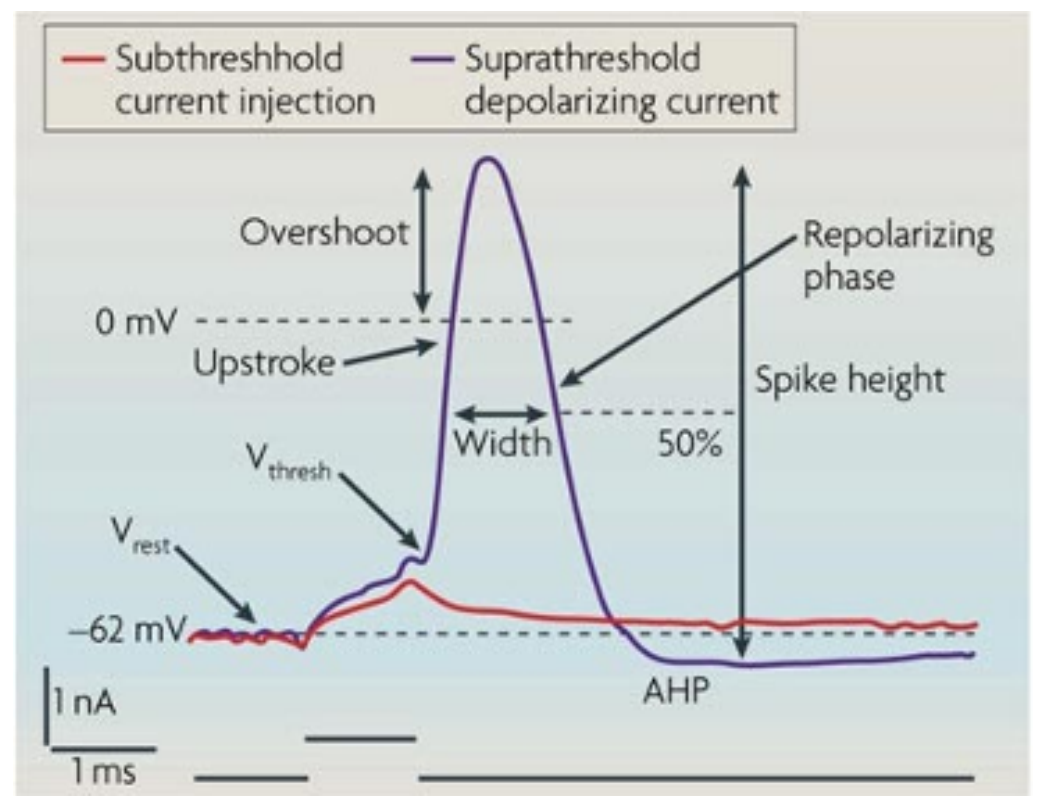

Figure 2.1: Anatomy of an action potential: The action potential in the figure is recorded from a pyramidal neuron in CA1 region of a rat hippocampus and illustrates commonly measured parameters. The action potential (purple) was elicited by the injection of a just-suprathreshold current (black) and also the response to a subthreshold is given in the figure (red). ( $\mathrm{V}_{\text {rest }}$ :resting potential; $\mathrm{V}_{\text {thresh }}$ : voltage threshold; upstroke: "depolarizing" or "rising" phase. Its maximum is given at $0 \mathrm{mV}$; overshoot: peak relative to $0 \mathrm{mV}$; spike height: peak relative to either resting potential or the most negative potential reached during afterhyperpolarization; AHP: afterhyperpolarization; spike width: width at half-maximal spike amplitude. repolarizing phase: "falling phase" or "downstroke". It has a much more slower velocity than rising phase.) Adapted from Bean [15] 
shape of AP are generally more significant for the presynaptic terminals, where small changes in the onset rapidness, amplitude or width can trigger drastic changes in the timing of calcium entry and therefore, in the postsynaptic currents.

The AP shape may also change for different regions of the neuron. The initiation site of the APs and its generation dynamics are critical parameters to understand the reason of this variability in the shape. The initiation site is commonly accepted to be 30-50 $\mu \mathrm{m}$ away from soma (Coombs et al. [44],Coombs et al. [45]; Häusser et al. [75]; Colbert and Johnston [38]; Stuart et al. [173]; Martina et al. [119]; Colbert and Pan [39]; Palmer and Stuart [139]; Khaliq and Raman [94]; Shu et al. [164]; Kole et al. [97]; Kole and Stuart [99]; Clark et al. [37]; Schmidt-Hieber and Bischofberger [159]) and this distance is enough for the shape of AP in the soma to show clear effects due to non-uniformity of potential (Shu et al. [164]). Also, the threshold potential for an AP shows variability (see section 2.2.2) for different neuronal regions.

\subsubsection{The Onset}

Considering the AP as a tool to transmit information, probably the most important one of its phases would be the onset, which give an indirect measure of the capability of a neuron to respond to fast-changing fluctuations in the stimulus (Fourcaud-Trocme et al. [63]; Naundorf et al. [132]). A very sharp onset, or a "kink", would mean that the delay between the trigger and the generation of the AP as the response is very small. Therefore, this neuron can phase-lock with fast-changing stimuli.

As the underlying dynamics of the AP has a time course of less than a ms, I will here mention only the currents that are activated at the initial phase of an AP and at the subthreshold potentials.

\section{The Subthreshold Currents and $\mathrm{Na}^{+}$Current at the Onset}

The membrane potential fluctuations at the subthreshold level are most significant when responding to the rapid fluctuations in the stimulus. The underlying ionic currents in the central neurons include A-type $\mathrm{K}^{+}$current $\left(I_{A}\right)$, Kv1-mediated $\mathrm{K}^{+}$ currents $\left(I_{D}\right)$, the current known as $I_{h}$ carried by hyperpolarization-activated cyclic nucleotide-gated (HCN) channels, steady-state "persistent" $\mathrm{Na}^{+}$currents $\left(I_{N a P}\right)$, and the current mediated by T-type (low-voltage-activated) $\mathrm{Ca}^{2+}$ channels.

A-type current was described in molluscan neurons in the classic papers by Connor and Stevens [40],Connor and Stevens [41]. This currents are both activated and inactivated at subthreshold potentials and they support repetitive firing at low fre- 


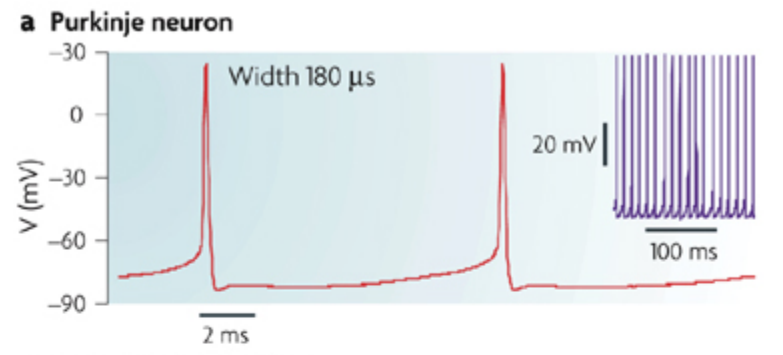

b CA1 pyramidal neuron
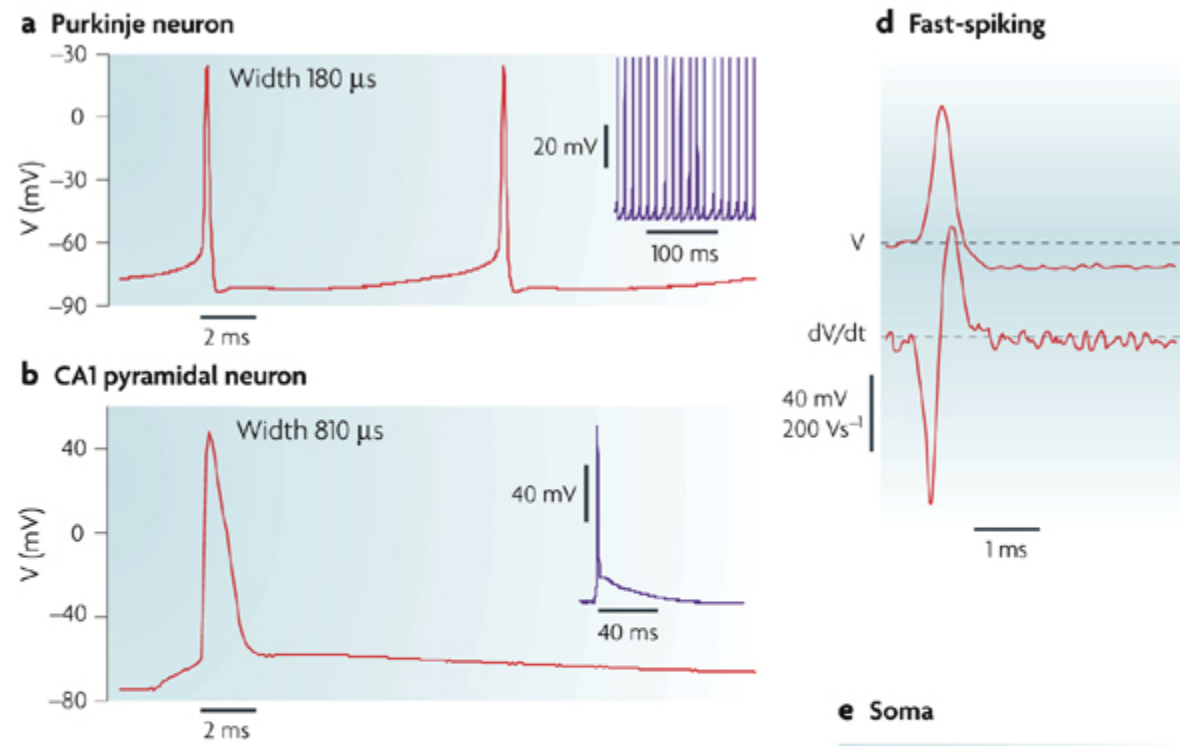

Regular-spiking

c Dopamine neuron
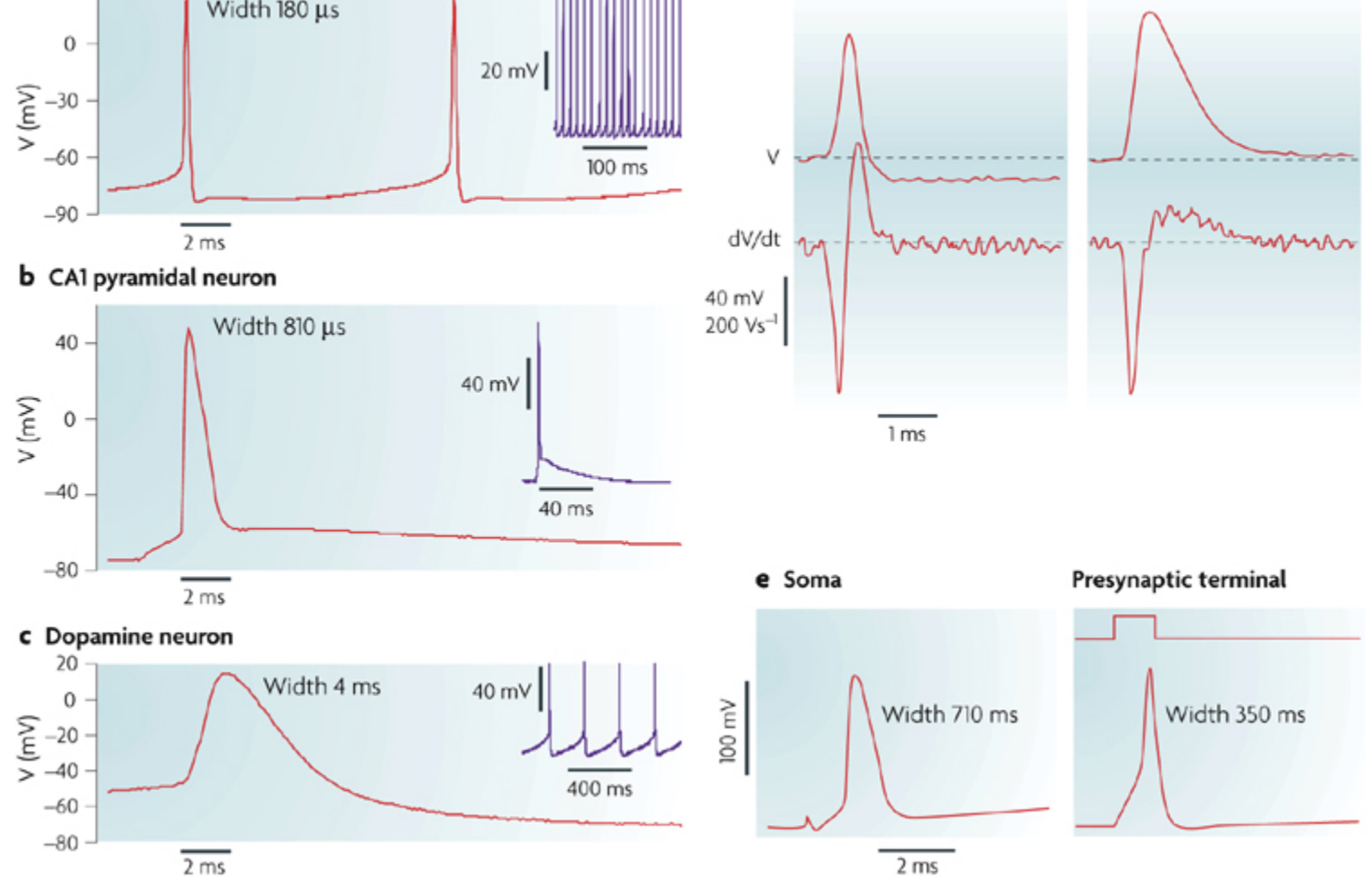

Presynaptic terminal

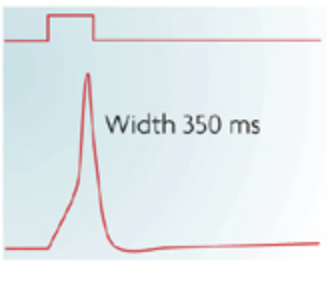

Figure 2.2: Diversity of APs in central neurons.(a) Spontaneous APs in an acutely dissociated mouse cerebellar Purkinje neuron; (b) AP in a hippocampal CA1 pyramidal neuron in brain slice; (c) spontaneous APs in a midbrain dopamine neuron; (d) illustration of APs from fast-spiking and regular-spiking cortical neurons; (e) different AP widths in the soma of dentate gyrus granule neurons and in the mossy fiber bouton, a presynaptic terminal made by a granule neuron. Adapted from Bean [15]. 
quencies. $I_{D}$ produces a subthreshold current that function similarly to $I_{A}$. T-type $\mathrm{Ca}^{2+}$ originate from CaV3 family channels, and their most significant function is the production of rebound bursting following hyperpolarization.

Fast-inactivated, transient $\mathrm{Na}^{+}$currents display a significant function in the initiation and propagation of APs (Hodgkin and Huxley [83]; Stuart and Sakmann [172]). In addition to these currents, at the subthreshold potential range, another more slowly inactivating, TTX-sensitive "persistent" $\mathrm{Na}^{+}$current $\left(I_{N a P}\right)$ is found in most neurons (Gutnick and Crill [74]; Astman et al. [11]). As the location of origin for $I_{N a P}$, the regions near soma and axon in layer V pyramidal neurons (Stuart and Sakmann [172]) and in CA1 pyramidal neurons (Andreasen and Lambert [6];Yue et al. [188]) were suggested. However, it was shown that the dendrites also contain the channels that generate $I_{N a P}$ (Lipowsky et al. [111]; Mittmann et al. [126]; Magistretti et al. [116]).

$I_{N a P}$ is mainly generated in the axon (Astman et al. [11]). However, it is not clear whether this reflects higher channel density, higher probability of late openings of the individual channels, or both. If it is assumed that the somatic and axonal channels have the same properties, then the experimentally observed $I_{N a P}$ magnitude would require the axonal channel density to be 500-1000-fold higher than the somatic density (Astman et al. [11]). However, these channel densities are proven to be unrealistic by experimental studies (Catterall [35]; Colbert and Johnston [38]; Colbert and Pan [39]; Engel and Jonas [55]; Schmidt-Hieber and Bischofberger [159]) as also explained below. Therefore, the altered biophysical properties of $\mathrm{Na}^{+}$channels in that region becomes more reasonable. Indeed, $I_{N a P}$ exhibits some unique properties, such as failing to exhibit fast inactivation, and this may be due to the differences in channel subunit composition. Caldwell et al. [30] showed that predominately axonal $\mathrm{Na}_{V} 1.6$ channels enter the non-inactivating gating mode more frequently and produce more persistent current than their relatives localized in soma and dendrites (Raman and Bean [148]; Rush et al. [154]). Still, the possibility that regional specificity of local regulatory systems (Cantrell and Catterall [32]) or distinctive channel arrangement relative to intracellular and extracellular matrices (Srinivasan et al. [169]). Indeed, recent studies reported that different $\mathrm{Na}_{V}$ channels, that are abundantly found in the AIS, do not necessarily co-localize (Hu et al. [86]; Rush et al. [154]). Furthermore, it was suggested that this distinct localization contributes to the AIS function. These results will be discussed in more detail in section 2.2 .

It was also shown that there is a $6-7 \mathrm{mV}$ shift in the voltage-dependence of $g_{\mathrm{Na}}$ in axon compared to somatodendritic $g_{N a}$ (Colbert and Pan [39]; Fleidervish et al. $[60])$.

Alternatively to the high channel density hypothesis, the sudden onset of phaseplot at threshold can be interpreted to suggest cooperative $\mathrm{Na}^{+}$gating (Naundorf 
et al. [132]). It was also shown that classic Hodgkin-Huxley models (see section 4.3) fail to reproduce the fast onset slopes of $>1 / 20 \mathrm{~ms}-1$ at $10-15 \mathrm{~V} / \mathrm{s}$ in cortical neurons. However, this hypothesis was strongly rejected by some researchers who suggested the distal, axonal initiation combined with the back-propagation into the soma could explain the phenomenon (Shu et al. [164]; McCormick et al. [123]; Bean [15]; Kress et al. [101]). A recent study shows, however, that this does not necessarily explain the fast onset slopes (Baranauskas et al. [14]). The cooperative gating hypothesis will be discussed in detail in chapter 7 .

\subsubsection{The Energy Budget}

The generation and transmission of the electrical signals constitute the largest part of the overall energy budget in the mammalian brain. It was shown in the previous studies that AP initiation is an important part of the total energy consumption and even, for invertebrates, e.g., the squid, it requires much more ATP then the actual synaptic transmission of the information. There are three basic reasons for such high energy consumption during an AP generation (Hodgkin [82]; Crotty et al. [46]; Sengupta et al. [161]):

- A robust signal often requires a charging of the membrane capacitance by more than $50 \mathrm{mV}$ up to the AP peak.

- The overall distance and the area that the AP should travel and invade is large and in some areas, such as very thin dendrites and the axon, densely packed. This also increases the amount of potential required to charge the membrane capacitance.

- Due to the overlapping $\mathrm{Na}^{+}$and $\mathrm{K}^{+}$currents in the opposite directions, the minimum flux required to charge the membrane is exceeded and the energy efficiency decreases.

Therefore, the structure of the neural code and circuit is affected in a way to optimize the energy usage to transmit APs. The sparse codes and redundancy reduction to transmit more information with less energy, the more efficient wiring of the circuit so that the AP does not need to travel for longer distances, and the reduction in the energy consumption for the production of APs by evolution of required biophysical properties are some of the examples of such biological optimizations (Niven and Laughlin [135]; Alle et al. [2]; Carter and Bean [34]).

$\mathrm{Na}^{+}$efficiency (or in other words, the ratio between the capacitive minimum $\mathrm{Na}^{+}$ load and the total $\mathrm{Na}^{+}$load) is an important factor in the AP energy cost. Carter and Bean [34] suggested that AP shape is a primary determinant of the differences 
in $\mathrm{Na}^{+}$entry efficiency; however, Sengupta et al. [161] pointed the $\mathrm{Na}^{+}$gating kinetics, more specifically the inactivation of $\mathrm{Na}^{+}$channels, as a more effective factor. Their studies on various single-compartment models representing a range from invertebrate to vertebrate neurons showed that shifting the core parameters such as the total conductance, activation time constant and inactivation time constant of voltage-gated $\mathrm{Na}^{+}$conductance and activation time constant of the voltage-gated $\mathrm{K}^{+}$ yields to move the models to the minima in their physiological conditions. These shifts in the parameters commonly led to a reduction in the overlap of $\mathrm{Na}^{+}$and $\mathrm{K}^{+}$ currents, which was thought to be responsible of the high energy consumption during AP generation. On the other hand, the amplitudes of changes required in the model parameters varied, which was also in agreement with the fact that the physiological efficiency of the neurons also vary (Figure 2.3). For example, the squid axon was shown to have an efficiency around 25\% (Hodgkin [82]) and by comparison, some of the mammalian neurons approach to the ideal efficiency of $100 \%$ by approaching the theoretical minimum $\mathrm{Na}^{+}$load (Alle et al. [2]; Carter and Bean [34]).

The high energy consumption during the AP generation puts a constraint on the amount of energy that will be spared for the information processing by synapses and prevents higher rates of processing. As an example, one can think of the axonal mass in the cerebral cortex (Attwell and Laughlin [12]; Sengupta et al. [161]). In a cubic millimeter of gray matter, there is approximately $3 \mathrm{~km}$ of excitable axons, most of which are pyramidal neurons. Each pyramidal neuron uses approximately $4 \mathrm{~cm}$ of 0.3 $\mu \mathrm{m}$ diameter axon collateral to transmit information via its 10,000 output synapses. It was estimated that, when a neuron fires an AP to drive these synapses, it acquires a minimum capacitive load of $2.36^{*} 10^{8} \mathrm{Na}^{+}$ions, that would require approximately $8^{*} 10^{7}$ ATP molecules to eject (Attwell and Laughlin [12]). Considering that this calculation is only for one AP, the rest of the calculations for the massive number of neuronal extensions and synapses underlines the cost of information processing in the brain.

The parameters used in the calculations mentioned above were adopted for squid axon, which is only $25 \%$ efficient and the result was that AP generation should consume significantly more energy than the synaptic transmission. However, in the study by Sengupta et al. [161], this value was calculated again using the parameters from mouse cortical pyramidal neurons, which are $80 \%$ efficient, and it was revealed that the level of synaptic trafficking that can be supported by the specific metabolic rate of gray matter was $60 \%$ larger, which would mean the increase in the supported average firing rate from $4 \mathrm{~Hz}$ to $6.8 \mathrm{~Hz}$, and that AP efficiency shifted the balance of energy expenditure from AP generation to synaptic transmission (Figure 2.4). 


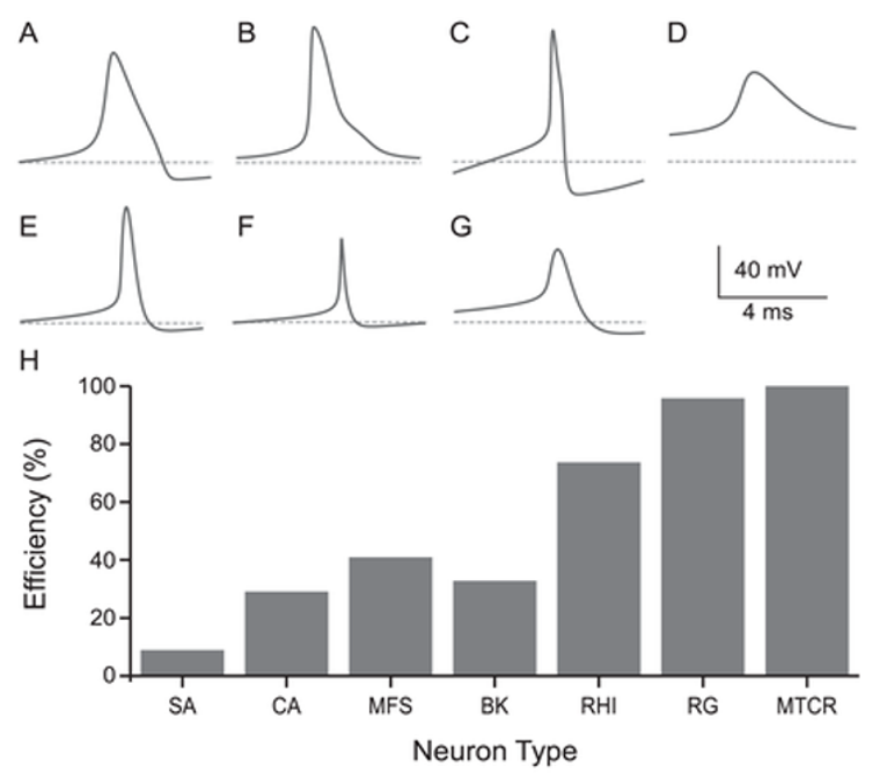

Figure 2.3: AP energy usage in seven models from vertebrates and invertebrates .The shape of APs in single compartment Hodgkin-Huxley type models from (A) the squid giant axon (SA), (b) crab motor neuron axon (CA), (c) mouse fast-spiking neuron (MFS), (d) honeybee Kenyon cell (BK), (e) rat hippocampal interneuron (RHI), (f) rat granule cell (RG) and (g) mouse thalamo-cortical relay neuron (MCTR). The dashed line indicates the resting potential of each model. (h) The efficiency of AP from each model. Error bars show the effect of changing the peak conductances of the voltage-gated ion channel by $\pm 5 \%$ on AP energy co-efficiency (see Sengupta et al. [161]). Adapted from Sengupta et al. [161].

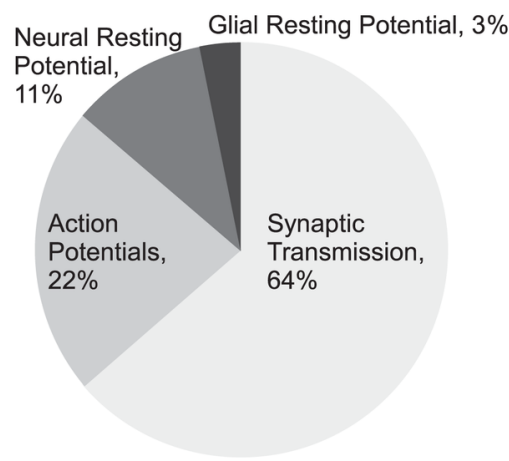

Figure 2.4: Energy budget for signaling in the gray matter of the rat brain. (For details, see Sengupta et al. [161]). Adapted from Sengupta et al. [161]. 


\subsection{Unique Properties of Initiation Site}

As briefly mentioned above, the AP is commonly accepted as initiated at the axon initial segment (AIS) of the neuron (Palmer and Stuart [139]; Coombs et al. [44],[45]; Häusser et al. [75]; Colbert and Johnston [38]; Stuart et al. [173]; Martina et al. [119]; Colbert and Pan [39]; Palmer and Stuart [139]; Khaliq and Raman [94]; Shu et al. [164]; Kole et al. [97]; Kole and Stuart [99]; Clark et al. [37]; Schmidt-Hieber and Bischofberger [159]). The first time that AIS was hypothesized to be site of AP initiation was in the paper by Araki and Otani [9]. At that time, other locations such as the axon hillock (Bishop [17]) or soma (Fatt [58]) were also suggested as the initiation site. Later on, Coombs et al. [44],[45] also hypothesized that the AP initiates at the AIS; but still, the first direct evidence came from the study by Edwards and Ottoson [54] on the neuron of lobster stretch receptor. They also showed for the first time that AP propagates orthodromically and antidromically. With the application of electron microscopy, Conradi [43] and Palay et al. [138] provided the first detailed anatomical description of AIS. An up-to-date list of reports on the axonal initiation sites is given in Figure 2.5.

Previous studies suggested that the APs are locally initiated at dendritic compartments when exposed to strong depolarizations, which then propagate to soma and AIS (Gasparini et al. [65]; Golding and Spruston [68]; [108]; Losonczy and Magee [113]; Schiller et al. [158]). However, Shu et al. [164] claimed oppositely that during epileptiform activity after disinhibition in vitro, fast APs were always initiated in AIS of neocortical pyramidal cells, followed by back-propagation into the soma and apical dendrites. This seemingly contradictive results might be explained by the fact that the basal and nearby oblique apical dendrites of cortical pyramidal cells receive 75-95\% of their excitatory of their synaptic inputs in neocortex, within $200 \mu \mathrm{m}$ from soma (Larkman [105],[106],[107]). These extensions are electrotonically closer to AIS then to the distal apical dendrites, which may result in the AIS initiation of AP after the arrival of large EPSPs. Still, the dendritic initiation can not be ruled out as that may be physiologically important, e.g., in the modulation of the overall pattern of discharge. However, nearly all of the fast spikes are generated in AIS (Shu et al. [164]).

The AP initiation AIS has a number of functional implications. For instance (Clark et al. [37])

- the AP generating mechanisms may be electrically isolated from soma/dendrite, allowing the AIS to serve as the single site of synaptic integration of all the inputs arriving to different parts of dendrites (Mainen et al. [117]);

- the localization AP generating machinery to a small subcellular compartment may allow for targeted and potentially powerful modulation of AP initiation by local influences such as inhibition as in the case of Chandelier cells; 


\begin{tabular}{|c|c|c|c|c|}
\hline The site name & Organism & Cell Type & Myelin & Author (s) \\
\hline AIS & Cat & Spinal cord motoneuron & Y & Coombset al., 1957 \\
\hline AIS & Cat & Spinal cord motoneuron & Y & Fuortes et al., 1957 \\
\hline AIS & - & $\begin{array}{l}\text { Dopaminergic neuron } \\
\text { (substantia nigra) }\end{array}$ & Y & Hausser et al., 1995 \\
\hline NR & $\begin{array}{l}\text { Rat(2-8 } \\
\text { weeks) }\end{array}$ & Subicular pyramidal neuron & Y & $\begin{array}{c}\text { Colbert and Johnston, } \\
1996\end{array}$ \\
\hline AIS & Rat & Neocortical pyramidal neuron & Y & Stuart et al., 1997a \\
\hline AIS & Rat & Spinal cord motoneuron & Y & Wolffet al., 1999 \\
\hline $\begin{array}{l}\text { Somatodendritic }{ }^{1} \\
\text { AIS }^{2}\end{array}$ & Rat & $\begin{array}{c}\text { Hippocampal CA1 GABAergic } \\
\text { interneuron }\end{array}$ & Y & Martina et al., 2000 \\
\hline NR & Rat & $\begin{array}{c}\text { Layer } V \text { neocortical pyramidal } \\
\text { neuron }\end{array}$ & Y & Colbert and Pan, 2002 \\
\hline NR & Rat & $\begin{array}{c}\text { Cerebellar Purkinje } \\
\text { neuron }\end{array}$ & Y & Clark et al., 2005 \\
\hline AIS & $\begin{array}{l}\text { Mouse (14- } \\
28 \text { days) }\end{array}$ & $\begin{array}{c}\text { Cerebellar Purkinje } \\
\text { neuron }\end{array}$ & Y & $\begin{array}{l}\text { Khaliq and Raman, } \\
2006\end{array}$ \\
\hline Distal AIS & $\begin{array}{l}\text { Rat(3-5 } \\
\text { weeks) }\end{array}$ & Layer V pyramidal neuron & Y & $\begin{array}{l}\text { Palmer and Stuart, } \\
2006\end{array}$ \\
\hline AIS & $\begin{array}{l}\text { Rat }(12-18 \\
\text { days) }\end{array}$ & $\begin{array}{l}\text { Mesencephalic } \\
\text { trigeminal neuron }\end{array}$ & Y & Saito et al., 2006 \\
\hline AIS & $\begin{array}{l}\text { Ferret ( } 7-10 \\
\text { weeks) }\end{array}$ & Neocortical pyramidal neuron & Y & Shu et al., 2007 \\
\hline Distal AIS & Rat (22 days) & CA3 pyramidal neuron & $\mathrm{N}$ & $\begin{array}{l}\text { Meeks and Mennerick, } \\
2007\end{array}$ \\
\hline AIS & $\begin{array}{l}\text { Mouse } \\
\text { (adult) }\end{array}$ & $\begin{array}{c}\text { Dentate gyrus (DG) granule } \\
\text { neuron }\end{array}$ & Y & $\begin{array}{l}\text { Schmidt-Hieber et al., } \\
2008\end{array}$ \\
\hline Distal AIS & $\begin{array}{l}\text { Rat }(2-4 \\
\text { weeks) }\end{array}$ & $\begin{array}{c}\text { Cortical layer } V \text { pyramidal } \\
\text { neuron }\end{array}$ & Y & Kole et al., 2008 \\
\hline AIS & - & $\begin{array}{l}\text { CA3 pyramidal neuron } \\
\text { DG granule neuron }\end{array}$ & $\mathrm{N}$ & Kress et al., 2008 \\
\hline Proximal AIS & $\begin{array}{l}\text { Mouse } \\
\text { (adult) }\end{array}$ & Hippocampal mossy fiber & $\mathrm{N}$ & $\begin{array}{l}\text { Schmodt-Hieber and } \\
\text { Bischopberger, } 2010\end{array}$ \\
\hline AIS & & $\begin{array}{l}\text { Layer V neocortical } \\
\text { pyramidal neuron }\end{array}$ & Y & Fleidervish et al., 2010 \\
\hline
\end{tabular}

Figure 2.5: A brief summary of Literature on AP initiation site. The table gives examples from the literature about the AP initiation sites of various cell types and organisms. AIS = axon initial segment, $\mathrm{NR}=$ Node of Ranvier, $\mathrm{y}=$ yes(myelinated), $\mathrm{N}=$ no(unmyelinated). Notes : 1) under long threshold stimuli, 2) under brief stimuli. 

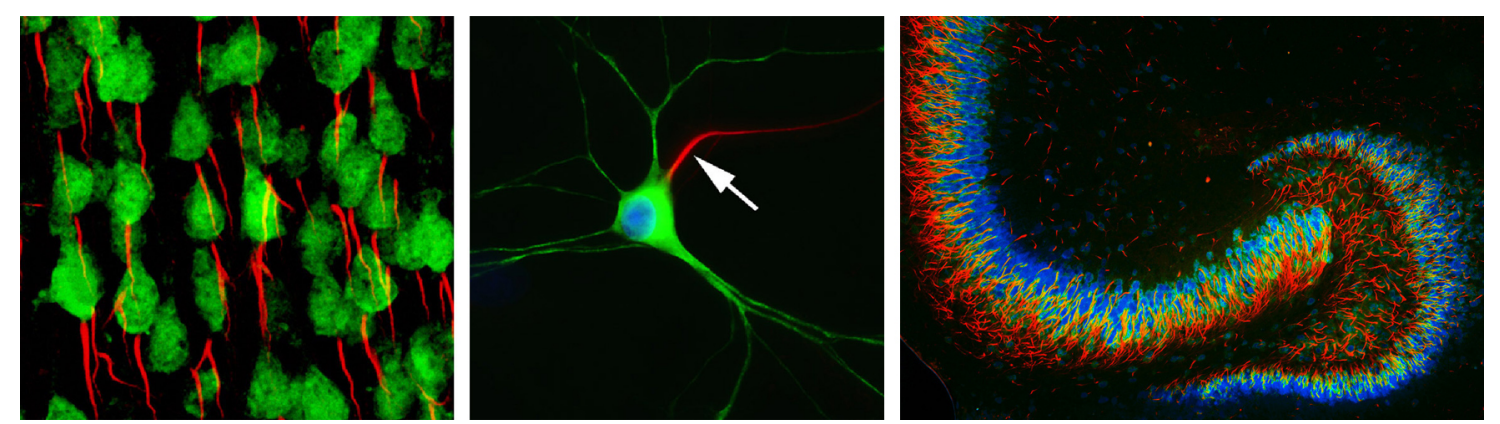

Figure 2.6: Axon initial segments staining.(Left) Specific enrichment of phosphorylated $\mathrm{I} \kappa-\beta \alpha$ (red) in the AIS of neurons in the rat cerebral cortex and (Middle) in a primary neuronal culture. The green labeling depicts the somatodendritic neuronal compartment. (Right) Specific enrichment of phosphorylated I $\kappa-\beta \alpha$ (red) in the AIS of an organotypic hippocampal slice culture. Adapted from Schultz et al. [160].

- charging the soma enough to promote back-propagation into the dendritic tree (Stuart et al. [173]).

\subsubsection{Molecular Structure of Axon Initial Segment}

As my project on cooperative AIS model (Part III) focus on the gating kinetics of the voltage-gated $\mathrm{Na}^{+}$channels in AIS, I will give a very brief picture of the molecular structure of this region. This underlying structure is very important for its unique functional implications.

The conical-like region between the soma and the axon was named as the axon hillock. This part was generally assumed as 5-15 $\mu \mathrm{m}$ length (Shu et al. [164]). AIS follows the axon hillock and it has a typical length of 17- $40 \mu \mathrm{m}$ (Palmer and Stuart [139]; Shu et al. [164]). The distal end of AIS, which is marked with the loss of submembraneous structures typical of the AIS and extracellular matrix level (Shu et al. [164]), was commonly associated with the beginning of myelination (Farinas and DeFelipe [57]; Inda et al. [90]; Palay et al. [138]).

AIS region of the neuron was proven to develop using elaborated molecular mechanisms to construct the formation of subdomains and the unique composition of ionic channels. This molecular mechanism is briefly given in Figure 2.7 (Grubb and Burrone [72]). This dense molecular structure was also observed as dense granular material underneath the membrane, microtubules in cytoplasm and scattered clusters of ribosomes (Farinas and DeFelipe [57]; Inda et al. [90]; Palay et al. [138]). This packed construction might serve as a barrage for large particles between soma and axon. 
(a)

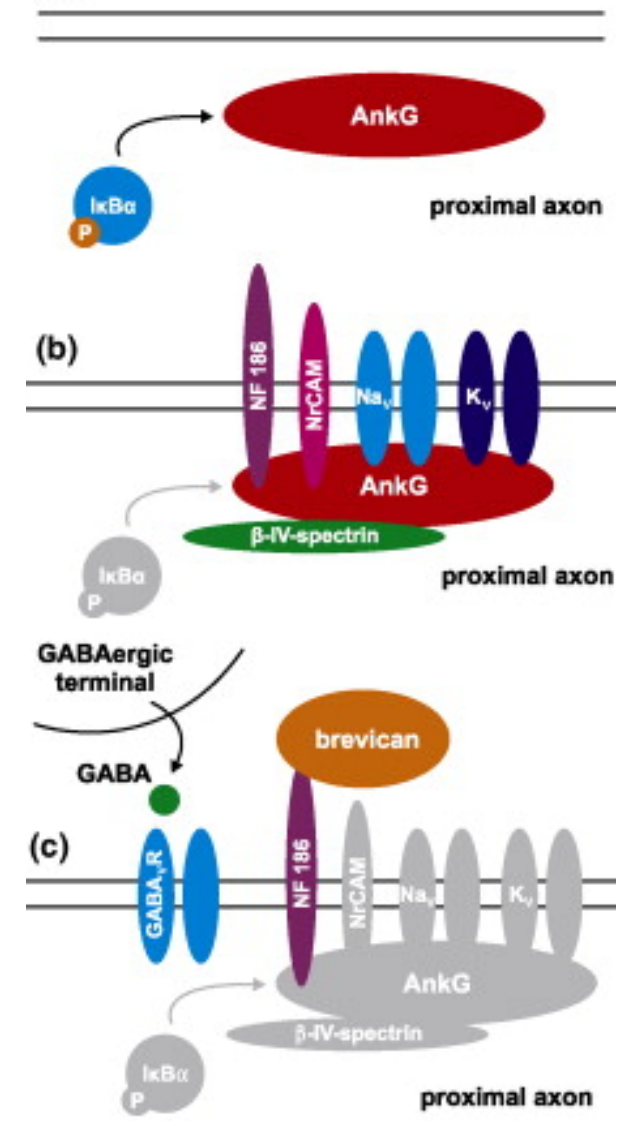

Figure 2.7: Simplified stages in AIS development.(a) During and shortly after axon specification, phosphorylated $\mathrm{I} \kappa \beta \alpha$ in the proximal axon is needed for localization of AnkG. (b) AnkG, now localized to a single band in the proximal axon, binds to and localizes $\beta \mathrm{IV}$-spectrin, $\mathrm{Na}_{V}$ and $\mathrm{K}_{V}$ channels and transmembrane proteins, among other molecules. (c) NF186 is then required for the development of a specialized brevican-containing AIS ECM and for the formation of AIS-specific GABAergic synapses. Adapted from Grubb and Burrone [72]. 
Two most important candidates for the specific functional role of AIS as the site of AP initiation are the biophysical characteristics and the density of the $\mathrm{Na}_{V}$ channels in that region. Recent studies suggested the existence of at least three isoforms : $\mathrm{Na}_{V} 1.6, \mathrm{Na}_{V} 1.1$ and $\mathrm{Na}_{V} 1.2$ (Clark et al. [37]; Grubb and Burrone [72]; Hu et al. [86]; Rush et al. [154]). A recent study also investigates the sublocalized distribution of yet another isoform $\mathrm{Na}_{V} 1.2$ (Hu et al. [86]). $\mathrm{Na}_{V} 1.6$, which is the most common $\mathrm{Na}^{+}$channel isoform in the nervous system, is also probably the most predominant isoform at the AIS (Boiko et al. [18]; Ogawa and Rasband [136]). In the AIS regions of fast-spiking, GABAergic inhibitory interneurons of cortex, $\mathrm{Na}_{V} 1.1$ isoform can also be found (Yu et al. [187]; Ogiwara et al. [137]). The loss-of-function mutation in the gene encoding this channel protein produce epilepsy (Ragsdale [143]), which makes the channel also a target for drug research. The $\mathrm{Na}_{V} 1.1$ channels are usually tightly localized at a small band on the very proximal edge of the AIS (Van Wart et al. [181]; Lorincz and Nusser [112]; Duflocq et al. [51]). On the other hand, $\mathrm{Na}_{V} 1.6$ distribution does not co-localize with $\mathrm{Na}_{V} 1.1$ isoforms and instead, increase in density towards the distal end of the AIS (Van Wart et al. [181]; Lorincz and Nusser [112]). A recent study described a similar sublocalization between proximal $\mathrm{Na}_{V} 1.2$ and distal $\mathrm{Na}_{V} 1.6$ isoforms in the AIS of cortical pyramidal neurons (Hu et al. [86]). Hu et al. [86] combined patch-clamp recording and mathematical simulations to show that the $\mathrm{Na}_{V}$ 1.2-containing and $\mathrm{Na}_{V}$ 1.6-containing portions of the AIS display distinct functional roles. However, the patch-clamp recording was performed on a bleb, which might disrupt the cytoskeletal structure underneath the AIS membrane. The authors justified their results with the claim that the recordings from this bleb, indeed, indicate the real profile of AIS APs and channel densities according to the results of their immunohistochemistry experiments. It was also shown in this study that the low voltage threshold of $\mathrm{Na}_{V} 1.6$ channels makes the distal AIS the site of AP initiation. This role of distal AIS was supported by the findings from other recent imaging, recording and modeling studies (Kole et al. [97]; Hu et al. [86]; Palmer and Stuart [139]; Meeks and Mennerick [125]).

The rich $\mathrm{K}_{V} 1$ subfamily composition on AIS suggest the possibility of complex local modulation of AP generation and properties by these channels (Grubb and Burrone [72]). Members of this channel subfamily are particularly interesting because they are activated at subthreshold membrane potentials and thus, can influence AP initiation. Recent immunohistochemical evidence revealed that in certain cell types, $\mathrm{K}_{V}$ 1.1. and $\mathrm{K}_{V} 1.2$ channels also preferantially target the distal AIS. At this subdomain, they always co-localize with each other, and with $\mathrm{Na}_{V} 1.6$ (Lorincz and Nusser [112]). This important finding suggests that these channels can play a specialized role in the control of AP initiation.

$\mathrm{K}_{V}$ 2-mediated currents accounted for a large proportion of the delayed rectifier current measured at the soma and immunohistochemical staining for $\mathrm{K}_{V} 2.2$ isoforms 
revealed their enrichment at AIS. These channels are localized at a more proximal region of AIS relative to the $\mathrm{K}_{V} 1$ channels (Johnston et al. [92]). It was also recently suggested that $\mathrm{K}_{V} 2.1$, the other known member of the $\mathrm{K}_{V} 2$ subfamily, is expressed at the AIS of hippocampal and neocortical neurons (Sarmiere et al. [156]).

KCNQ $\left(\mathrm{K}_{V} 7\right)$ subfamily of $\mathrm{K}^{+}$channels are also found as clusters at the AIS of several cell types (Clark et al. [37]). These channels, like $\mathrm{K}_{V} 1$, operate in subthreshold potentials and mediate the current known as "M-type $\mathrm{K}^{+}$current". These slow-activating, noninactivating and voltage-dependent currents are commonly accredited as predominant regulators of neuronal excitability in many neuron types (Clark et al. [37]; Shah et al. [163]) and are shown to contribute to stabilizing of the resting potential and to limiting repetitive firing (Clark et al. [37]). KCNQ2 ( $\left.\mathrm{K}_{V} 7.2\right)$ and KCNQ3 $\left(\mathrm{K}_{V} 7.3\right)$ isoforms are abundant at the AIS in several neuronal populations including hippocampal and neocortical pyramidal cells (Pan et al. [140]).

Bender and Trussell [16] found in their study on dorsal cochlear nucleus neurons that $\mathrm{T}$ - and R-type voltage-gated $\mathrm{Ca}^{2}+$ are the underlying transient calcium currents that are evoked by repetitive firing. These currents were significantly larger in AIS than soma. Similar currents were also observed in other cell types, such as layer 5 pyramidal cells and cerebellar Purkinje neurons (Bender and Trussell [16]).

The structural properties of AIS makes it a more favorable site of initiation. For instance;

- The large capacity and conductance load of the soma and dendrites decrease with increased distance down the axon, thereby, decreasing the amount of current required to initiate an AP (Shu et al. [164]).

- This region is strongly affected by the summated membrane potential arriving from dendritic branches and soma (Shu et al. [165],[164]), and by its passive and active electrical properties.

\subsubsection{Threshold Potential}

The difficulty in estimating the threshold is primarily due to the variations in the methods of measuring. This methods include "by-eye" estimates to methods based on first, second or third order derivatives (Henze and Buzsaki [77]; Naundorf et al. [132]; Meeks and Mennerick [125]; Shu et al. [164]). Therefore, estimates of threshold potential differ from $-43 \mathrm{mV}$ to $-63 \mathrm{mV}$ (Kress et al. [101]). The value of threshold varies significantly also for different types of neurons, such as $-52 \mathrm{mV}$ for dentate granule neurons, which is $10 \mathrm{mV}$ less than the threshold measured in the same study for CA3 pyramidal neurons (Kress et al. [101]). 
The first suggestion of a lower threshold potential $\left(V_{t h r}\right)$ at AIS came from the pioneering work of Coombs et al. [44],[45]. They predicted a $15 \mathrm{mV}$ lower (more hyperpolarized) $V_{t h r}$ at AIS than soma. Many following studies also predicted a lower threshold and lower voltage-dependence in the activation of voltage-gated $\mathrm{Na}^{+}$ channels in axon compared to soma (Colbert and Johnston [38]; Colbert and Pan [39]; Fleidervish et al. [60]). On the other hand, a recent study by Kole and Stuart [99] reported a $V_{t h r}$ at AIS that was higher than the soma. They also underlined that, opposite to $V_{t h r}$, the current threshold $\left(I_{t h r}\right)$ was significantly lower at AIS. For a reasonable explanation of "low- $I_{t h r}$-but-high- $V_{t h r}$ " phenomenon, they further tested the effect of TTX. They suggested that the effect they observed were due to the high $\mathrm{Na}^{+}$density, but not the shifted voltage dependence; and, in result, they claimed that the membrane potential in AIS is boosted in prior to AP initiation by the same $\mathrm{Na}^{+}$channel that underly the AP generation. This leads to a seemingly higher $V_{t h r}$ at AIS. When the $V_{t h r}$ at soma was measured isolatedly, it was found that $V_{t h r}$ at soma was $20 \mathrm{mV}$ higher than $V_{t h r}$ at AIS.

In many cases, the threshold at the soma was taken as $V_{t h r}$; however, a realistic $V_{t h r}$ value can be obtained only if the threshold is measured at the site of initiation.

\subsubsection{Channel Density}

As also mentioned by Colbert and Johnston [38], there is a requirement of some critical assumptions previous to the estimation of the channel densities, i.e. at the axonal regions. For instance, the following features are assumed to be known in advance (Colbert and Johnston [38]; Fleidervish et al. [60]):

- The area of the patch,

- The probability of opening,

- The number of activable channels,

- Uniformity of density in the membrane (no clustering),

- Statistically independent gating of ionic channels,

- The specific binding of labeling to the ionic channel (in case of immunohistochemistry and confocal microscopy studies) .

Therefore, instead of a direct measure, a relative measure of channel densities for different regions of a neuron would be more reasonable, though not exactly precise.

Colbert and Pan [39] also suggested that the biophysical properties of axonal $\mathrm{Na}^{+}$ channels rather than a high density of those channels in the initial segment, are most 
likely to determine the lowest threshold for AP initiation. The required densities would be as high as 100-fold. Such very high densities, up to 500-1000-fold densities as required by the results of Astman et al. [11], were hypotesized in a theoretical study (Mainen et al. [117]); however, the experimental studies did not confirm such high densities. For instance, immunohistochemical studies indicate that $\mathrm{Na}^{+}$conductance, $g_{N a}$, may be as high as 10-fold than in AIS compared to soma (Catterall [35]; Angelides et al. [7]). A freeze-fracture found that the total number of particals in initial segment is only 3-fold of that in soma (Matsumoto and Rosenbluth [121]). Further studies approved this early finding (Colbert and Johnston [38]; Colbert and Pan [39]; Kole et al. [97]; Fleidervish et al. [60]). Fleidervish et al. [60] showed the ratio of somatic and axonal $\mathrm{Na}^{+}$channel densities measurements on $\mathrm{Na}^{+}$flux density. This measure give a ratio of 3:1 axon-to-soma. However, it was not possible to directly take this ratio as the channel density ratios in two regions, as that would require the shape of APs and the temporal kinetics of underlying channels to be the same. They showed that the time to peak is $20-30 \%$ briefer in AIS, which means there will be a $\mathrm{Na}^{+}$flux residue at the same time window compared to soma. Therefore, the ratio would be even less than 3-fold channel density in AIS compared to soma.

It is essential to mention that when measuring channel density using immunohistochemistry, the probability of overestimation due to non-specific binding can not be ruled out (Fleidervish et al. [60]).

\subsubsection{Plasticity and Modulation of Excitability}

It is known that the wiring of the brain is altered with experience. Up to date, most of these changes were known to occur in the synaptic regions. Recent studies also reported a more direct regulation : the plasticity of the AIS region also contributes to the overall rearrangement and this contribution is very important considering that any modulation on the excitability of this region would directly affect the generation dynamics of the response.

Two of the recent studies provided very important information on AIS plasticity. On the hippocampal neurons, it was shown in vitro that the increased input density decreases the excitability of AIS region by translocating it more distally (Grubb and Burrone [71]). This results were obtained from two different experimental designs: (1) To mimic increased neuronal activity, the extracellular $\mathrm{K}^{+}$levels were chronically elevated. This approach provided evidence on the distal movement of AIS and all the AIS-specific molecular structure, creating a non-excitable "spacer" region. This spacer region was $21 \mu \mathrm{m}$ long. (2) To manipulate the neuronal activity with a more precise temporal control, they tranfected the culture to express Channelrhodopsin-2 (ChR2), which is a light activated ion channel (Boyden et al. [21]). The use of light 
stimuli provided the opportunity to directly trigger spiking in the desired region. The results obtained with the high-frequency bursts of light further supported the first finding - the distal translocation of AIS by increasing neuronal activity- whereas the long-term, regular, low-frequency light stimuli at $1 \mathrm{~Hz}$ did not have an impact. Grubb and Burrone [71] also showed that blocking of L- and T-type $\mathrm{Ca}^{2+}$ channels prevent AIS from moving, sugggesting an activity-dependent Ca2+-control on AIS plasticity.

The in vitro findings were justified by the in vivo studies of Kuba et al. [103]. Previously, they showed that auditory coincidence detection is enhanced by the axonal site of spike initiation (Kuba et al. [102]). Using immunohistochemistry on the chick brainstem slices, they provided evidence about the dependency of AIS length on the characteristic sound frequency that each neuron processes. AIS extension towards soma was shown using Pan-Na ${ }_{V}$ staining on the neurons with low characteristic frequency $(0.4-1 \mathrm{kHz})$. On the other hand, $\mathrm{Na}_{V}$ channel expression started more distally in the neurons with middle $(1.0-2.5 \mathrm{kHz})$ or high $(2.5-4.0 \mathrm{kHz})$ characteristic frequency (Figure 2.8). Next, they tested the effects of hearing loss on AIS location (Kuba et al. [103]). Removing the cochlea from one-day-old-chicks led to loss of synaptic input to neurons in nucleus magnocellularis. Following the hearing loss, these neurons exhibited an elongation of AIS by up to 70\%. As these neurons lack dendrites, it was aso confirmed that the excitability change was limited to axon. The authors also showed that there is an increase in the total $\mathrm{Na}^{+}$current in the axon although there is no change in the channel density or subtype composition. Therefore, they suggested that this increase was due to the expansion of AIS. This kind of change would obviously imply that smaller current input would be sufficient to trigger response and therefore, the reduced synaptic drive would be compensated after hearing loss.

Analyzing the two studies, Gründemann and Häusser [73] pointed also some unanswered questions in the studies. For instance, both studies were restricted to either developmental or pathological long-term manipulations and it is still not known whether the AIS plasticity is a normal phyiological mechanism that could dynamically regulate excitability. If so, the individual preference of neurons in specific network states and brain areas on either the expansion or displacement of AIS is still to be investigated. Furthermore, both of these groups left an unanswered question about the effect of AIS changes on integration of synaptic inputs. This is particularly important as the AIS region is known to be modulated by GABAergic synapses. It is yet to be answered whether these synapses also displace with AIS. Although the $\mathrm{Ca}^{2+}$-related mechanisms seem to be responsible, the details of the underlying molecular dynamics of this kind of plasticity is also still unknown. 


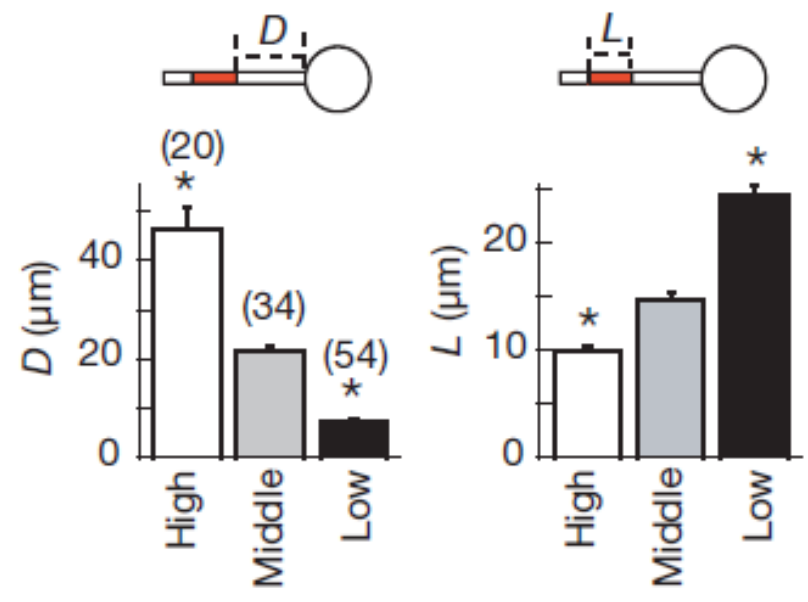

Figure 2.8: The location of $\mathrm{Na}_{V}$ channel clustering in the axon for neurons with different characteristic frequencies. Left: Distance $D$ and Length $L$ of $\mathrm{Na}_{V}$ channel clusters as revealed by Pan- $\mathrm{Na}_{V}$ staining for high, middle and low characteristic frequencies. Error bars indicate standard errors and. Asterisk, $\mathrm{P}<0.01$ compared with moddle characteristic frequency. Numbers in parentheses are the numbers of cells. Adapted from Kuba et al. [102]. 


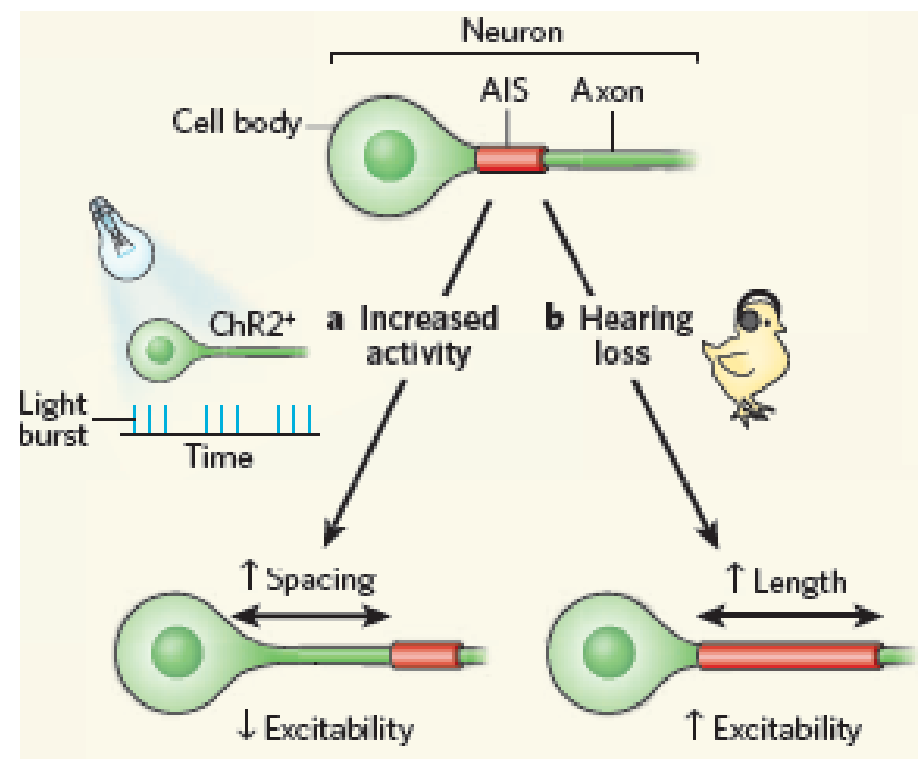

Figure 2.9: Intrinsic plasticity on AIS. (a) Grubb and Burrone [71] showed that, in cultural hippocampal neurons expressing light-activated ChR2, bursts of activity triggered by light lead to a calcium-dependent movement of AIS away from soma. Consequently, neuronal excitability is reduced. (b) Kuba et al. [103] found that, in neurons from the nucleus magnocellularis of chicks, deafness - and thus loss of sensory input - caused by removal of the cochlea increases the length of AIS, leading to corresponding compensatory increase in neuronal excitability. Adapted from Gründemann and Häusser [73]. 
28 Chapter 2: Initiation of the individual electrical signals 


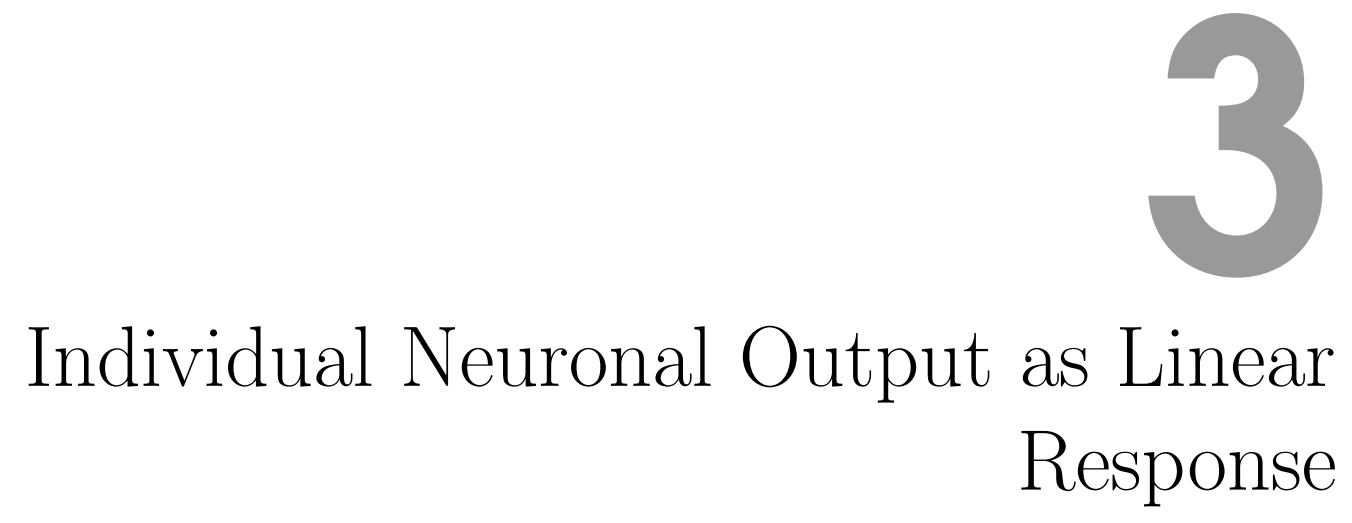

\subsection{Linear Response Theory}

Each individual neuron serves as a unit in a complex signal processing system, which filters and transmits the input as output to the other neurons. This signal processing is complicated and non-linear in most cases, however, when the neuron is driven with nominal parameters, it acts as a linear time-invariant unit. Therefore, its characteristic response functions can be treated as transfer functions and analyzed accordingly.

The principle of coding in a population, which consists of neurons with low average firing rates, was formulated as the linear response theory of neuronal populations by Knight [95],[96]. This theory in general explained how these populations could encode in response to fast-changing stimuli. Later on, Gerstner [66] generalized the theory by the application of artificial noise models. More sophisticated cortical neuron models with the application of more realistic synaptic input currents then followed (Brunel et al. [23]; Lindner and Schimansky-Geier [110]; Fourcaud and Brunel [61]; Fourcaud-Trocme et al. [63]; Naundorf et al. [131]). When constructing such synaptic input, one should consider the Poisson-like distribution of individual realizations that each neuron of a certain population receives. Therefore, the input can be considered as two parts: a mean input current and a Gaussian noise in addition, which can be time-correlated (colored noise) or not (white noise). Although synaptic filtering could be ignored, e.g., for an instantaneous synapse (as in white noise), a finite correlation time is more useful to construct a realistic noisy synaptic input (Fourcaud and Brunel [61]; Fourcaud-Trocme et al. [63]). As it is described later, both the mean and the variance of this noisy input was proven as significant determinants of linear response properties (Brunel et al. [23]; Lindner and Schimansky-Geier [110]; Fourcaud and Brunel [61]; Fourcaud-Trocme et al. [63]; Silberberg et al. [166]; Naundorf et al. [131]; Köndgen et al. [100]; Boucsein et al. [20]). 


\subsection{Determinants of Cutoff Frequency}

The input-output transformation performed by a neuron is classically characterized by its frequency-current (f-I) relationship. As important as the dependence of firing rate on the mean injected current, the neural response can be represented by terms of its gain of modulation of the firing rate as a function of the input frequency. This type of response were proven to have a low-pass characteristic with a decay $1 / f^{\alpha}$ when plotted in the double logaritmic plane (Fourcaud-Trocme et al. [63]; FourcaudTrocme and Brunel [62]; Naundorf et al. [131]; Köndgen et al. [100]; Boucsein et al. [20]). Until the inflection point, the response remains more or less stable. This input frequency, after which the response ability of a neuron decays sharply, is called as the cutoff frequency $\left(f_{\text {cut }}\right)$.

As explained in the next chapter, simple models such as linear integrate-and-fire (LIF) models were commonly used to study the dynamic response properties of real neurons. In the presence of white noise, LIF neurons behave as low-pass filters, with a $f_{\text {cut }}$ depending on the passive membrane time constant and the average firing rate of the neuron (Gerstner [66]; Brunel et al. [23]; Fourcaud and Brunel [61]). The gain of the filter decays as $1 / \sqrt{f_{I}}$, where $f_{I}$ is the frequency of the input current, and its phase shift reaches 45 at sufficiently large $f_{I}$. Additionally, in these studies, it was shown that the time correlations in the input noise improve the accuracy model response. However, Fourcaud-Trocme et al. [63] found that LIF model does not reproduce the response the conductance based models. The most significant finding of this study was the relationship between the cutoff frequency and the sharpness of the action potential onset. The effect of onset sharpness or the so-called threshold width $\left(\Delta_{T}\right)$ becomes more pronounced for increasing time correlation in the noise. Besides, the cutoff frequency was shown to have a linear dependence on the average steady-state firing rate $\left(\nu_{0}\right)$.

It is already known that the activation of $\mathrm{Na}^{+}$channels play a crucial role at the onset and upstroke of an action potential. Intuitively, the activation kinetics of $\mathrm{Na}^{+}$ channels should be one of the major determinants of the cutoff frequency. Their effect can be indirectly measured using the onset rapidness (or the so-called threshold width $\left.\Delta_{T}\right)$. For instance, using the measurements from several in vitro studies (Fleidervish and Gutnick [59]; Martina and Jonas [118]), Fourcaud-Trocme et al. [63] estimated a $\Delta_{T}$ in the range of 3-6 $\mathrm{mV}$. This would correspond to a $f_{\text {cut }}$ in the range of 40-50 $\mathrm{Hz}$ (Figure 3.1) for the results obtained from a more realistic integrate-and-fire (IF) model (exponential IF, EIF).

To sum up, the relations of $f_{\text {cut }}$ that are confirmed by both experimental and theoretical studies are

1. The neuronal gain decays as $1 / f^{\alpha}$, with $\alpha>1$, at high frequency, independently 


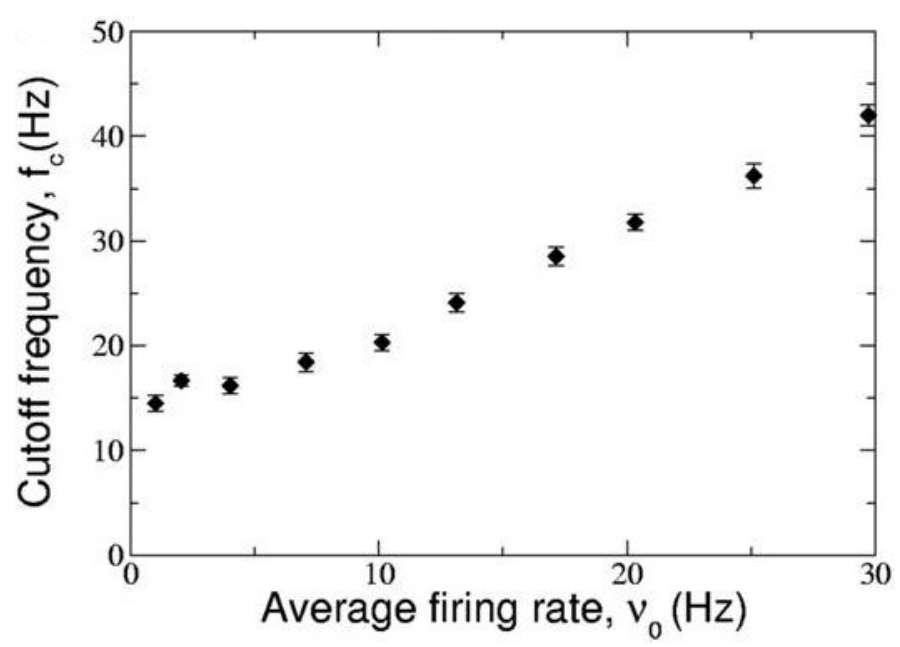

Figure 3.1: Cutoff frequency of the EIF filter in the high-noise regime as a function of the firing rate. The parameters of the EIF model were chosen to match the conductance-based Wang-Buzsaki model $\left(\Delta_{T}=3.48 \mathrm{mV}, \sigma_{V}=8 \mathrm{mV}\right)$. The cutoff frequency is approximately proportional to the average firing rate $\nu_{0}$ in the simulations with white noise. Adapted from Fourcaud-Trocme et al. [63].

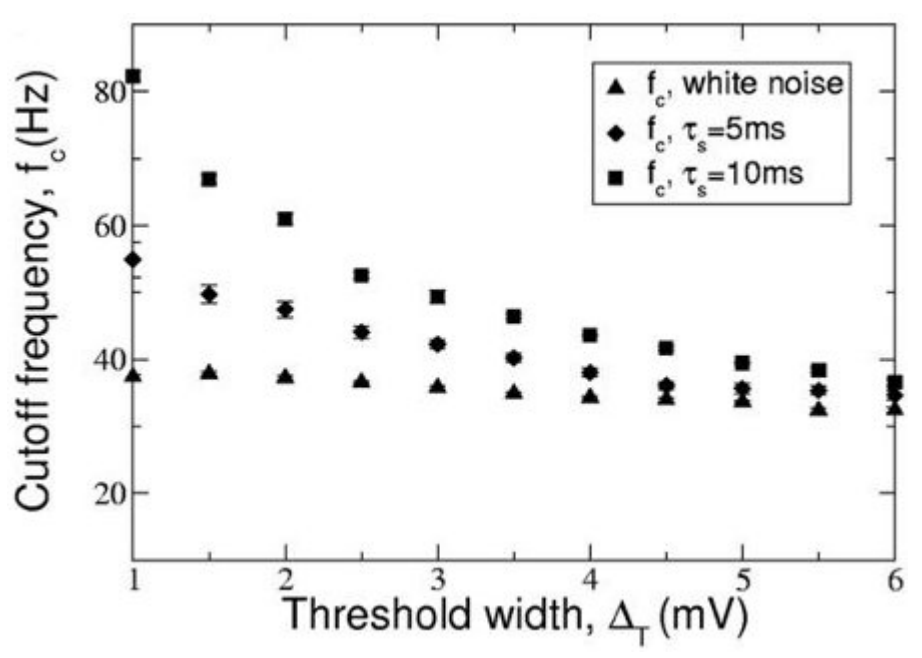

Figure 3.2: Cutoff frequency of the EIF filter in the high-noise regime as a function of threshold width, $\Delta_{T}$. The cutoff frequency depends weakly on the slope factor $\Delta_{T}$ for white noise but strongly increases when $\Delta_{T}$ decreases for colored noise $\left(\nu_{0}=24 \mathrm{~Hz}, \sigma_{V}=8 \mathrm{mV}\right.$. Values of the synaptic time constants are indicated in the legend). Adapted from Fourcaud-Trocme et al. [63]. 
of the characteristics of the input (Fourcaud-Trocme et al. [63]; Köndgen et al. [100]; Boucsein et al. [20]).

2. $f_{\text {cut }}$ increases with increasing $\nu_{0}$ (Fourcaud-Trocme et al. [63]).

3. $f_{\text {cut }}$ is linearly dependent on the onset sharpness (or inversely dependent on $\Delta_{T}$ ) in the presence of temporally correlated noise (Fourcaud-Trocme et al. [63]; Naundorf et al. [130], [131]).

On the other hand, there are some important points about the cut-off frequency that are still missing, such as (1) the method and the theory of extracting the population cut-off frequency from the single neuron response properties (i.e. the average firing rate and passive electrical parameters such as membrane time constant) and (2) the knowledge about the point, at which the population response can be dissociated from the single neuron response.

\subsection{Experimental Approach}

Various in vitro studies were performed previous to Köndgen et al. [100] and Boucsein et al. [20] for low frequency inputs $(<20 \mathrm{~Hz})$. Carandini et al. [33] found that the regular-spiking cortical cells exhibit a linear response to the injection of a broadband noisy input and this response is flat up to $100 \mathrm{~Hz}$. In some of these studies, the response properties of cortical neurons to stationary fluctuating inputs have been characterized over a limited range of frequencies (Chance et al. [36]; Rauch et al. [149]; Higgs et al. [80]; La Camera et al. [104]; Arsiero et al. [10]). However, Köndgen et al. [100] and Boucsein et al. [20]) provided a further extended experimental framework and presented striking findings as mentioned in the following sections.

The question of whether the neurons respond better to the stimuli, which are encoded in the mean input current, or to the stimuli, which modulate the variance of the input current is strongly debated. Silberberg et al. [166] addressed this question and presented a surprising finding that the average output rate could adapt almost instantly, with a time constant of $1 \mathrm{~ms}$, when the amplitude of the fluctuating input current was changed in a step-like fashion. In contrast, a step change in the mean input generated a slow response, with the time constant of $\approx 20 \mathrm{~ms}$.

The study adapted a direct approach to inject an in vivo-like current into the in vitro neuron : First, they obtained realistic synaptic currents from whole-cell voltageclamp in vivo using different levels of excitation. Then, they injected these fluctuating currents into the somata of pyramidal neurons in vitro. At a certain time point, either the mean or the variance of the input current was changed instantaneously. 


\section{A}

B
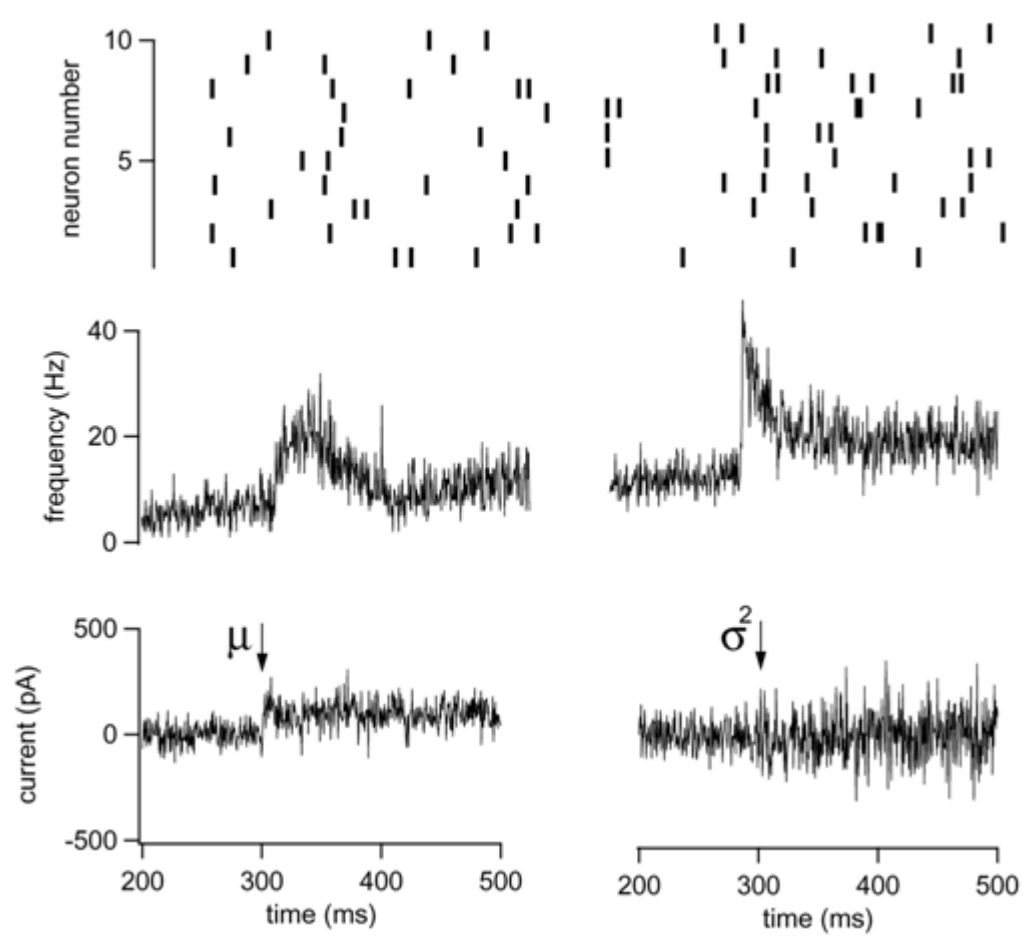

Figure 3.3: Response of neocortical neurons to abrupt changes in input parameters $\mu$ and $\sigma^{2}$. In each case, 4,000 different virtually white noise current traces (sampling interval in all of the experiments is $\tau_{\text {sample }}=0.25 \mathrm{~ms}$ ) were injected into a pyramidal neuron sequentially. (A) Bottom: An example of a single current trace injected. The arrow indicates the moment at which the amplitude of the mean current was increased. Middle: Histogram of the "population" response with a time bin of $1 \mathrm{~ms}$. At the transition point, the mean current was increased from 120 to 200 pA. Top: Raster plot of spike trains for 10 randomly chosen trials. Solid red lines show the stationary levels of the response before and after the transition. B: same as in $\mathrm{A}$, but the set of current time courses featured a change in the population variance from 22.5 to $90 \mathrm{pA}^{2}$.s. Histogram binning is $1 \mathrm{~ms}$. Adapted from Silberberg et al. [166]. 
Intuitively, their predictions could be justified considering a population of neurons simultaneously receiving a redundant excitation. The neurons in the population would be driven towards the threshold. Some of these neurons would fire and immediately afterwards would synchronously enter in the refractory period. For a rapid response, where the excitation amplitude would be massive, the population activity would be saturated temporarily. Therefore, the authors claimed that this can not be the way to transmit an analog signal. On the contrary, the increase in both excitation and inhibition, which would imply an increased population variance of the inputs, would trigger response only in a fraction of neurons. Thus, a large proportion of neurons would not reach to the threshold, although their membrane potential may remain fluctuating close to the threshold. This mechanism would both avoid the saturation of the population activity and maintain a certain level of subthreshold activity that can keep the neurons ready for continuous signals.

Köndgen et al. [100] confirmed the predictions of the theoretical studies FourcaudTrocme et al. [63] and Naundorf et al. [131]. A power law decay was observed after $f_{\text {cut }}$ as in previous studies, however, the decay was in the form of $1 / f^{\alpha}$ with $\alpha>$ 1. This value was less than or equal to 1 in the previous studies (Brunel et al. [23]; Fourcaud-Trocme et al. [63]; Fourcaud-Trocme and Brunel [62]; Naundorf et al. [130]).

Recently, it was shown that the modulations in the noise variance can be tracked significantly faster than the modulations in the main input (Boucsein et al. [20]). If the stimulus amplitudes are sufficiently high, extremely fast stimuli up to $1000 \mathrm{~Hz}$ can be tracked by the neuron.

The conclusions of Köndgen et al. [100] and Boucsein et al. [20] disproved the findings of Silberberg et al. [166] as well as the theoretical conclusions of [95],Gerstner [66] and Brunel et al. [23]. Although the background noise affects the neuronal dynamics at intermediate and low frequencies, neither the noise density nor the correlation time have an impact on the low-pass filtering profile (Köndgen et al. [100]). This conclusion was in aggreement with Fourcaud-Trocme et al. [63] and Naundorf et al. [130]. However, the in vitro estimates of cutoff frequencies were still higher than what was suggested by the conductance-based models (Köndgen et al. [100]; Boucsein et al. [20]; Fourcaud-Trocme et al. [63]).

Naundorf et al. [130] and Boucsein et al. [20] both found that neurons with finite action potential onset speed exhibit low-pass behavior for both additive and multiplicative modulation. Boucsein et al. [20] further confirmed these results for colored noise and a variety of stimulus amplitudes. In contrast to the study by Silberberg et al. [166], that used white noise and broad-band stimuli, Boucsein et al. [20] performed a frequency resolved analysis, that provided them with a more direct comparison for the theoretically predicted additive and multiplicative transfer functions. Furthermore, 


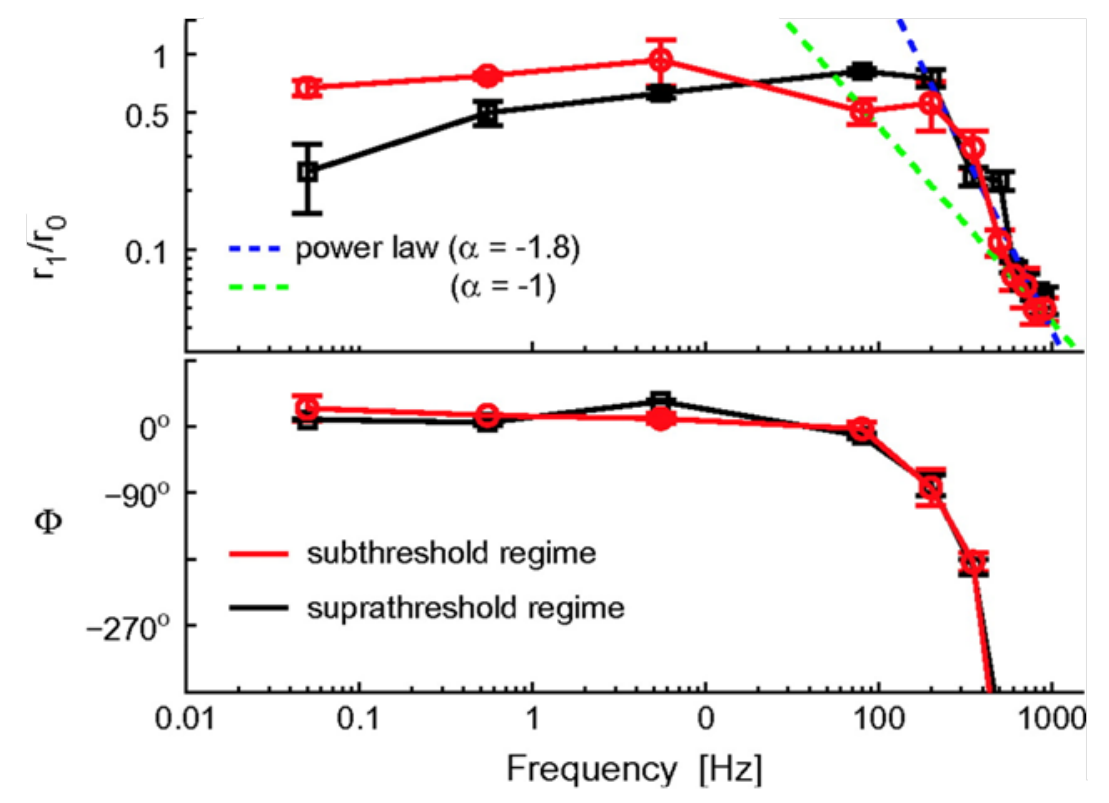

Figure 3.4: Modulation depth $\left(r_{1} / r_{0}\right)$ and phase-shift $(\phi)$ of the response to a noisy oscillatory input. The instantaneous firing rate $r(t)$ evoked by small sinusoidal currents over a noisy background revealed sinusoidal oscillations with amplitude $r_{1}$ and phase-shift $\phi$, around a mean $r_{0}$. Surprisingly, pyramidal neurons can relay fast input modulations, up to several hundred cycles per second. The high-frequency response behavior matches a power-law relationship with a linear phase-shift. Population $(\mathrm{n}=67$ cells) response was averaged across available repetitions and distinguishing between offset-currents above (suprathreshold regime) and below (subthreshold regime) the DC rheobase of the corresponding cell. Data points corresponding to distinct input modulation frequencies were pooled together in nonoverlapping bins with size $0.1-10 \mathrm{~Hz}$ (low frequencies) and 100-200 Hz (high frequencies). Error bars represent the standard error across the data points available (32- 25) for each bin. Markers shape and color identify the suprathreshold or weak-noise regime (black) and the subthreshold or strong-noise regime (red). $r_{0} \cong 20 \mathrm{~Hz}$ (i.e., $19.7 \mathrm{~Hz}$ ). Adapted from Köndgen et al. [100]. 
they observed that the response amplitude grew sublinearly with stimulus amplitude. That notion, however, was contradictive to the theoretical studies that predicted linear response for a large range of amplitudes (Brunel and Hakim [25]; Brunel et al. [23]; Fourcaud and Brunel [61]; Fourcaud-Trocme et al. [63]; Naundorf et al. [130]).

\subsection{Theoretical Approach}

Previous theoretical studies (e.g. Knight [95]; Gerstner [66]) emphasized the role of background noise in simplifying the neuronal response dynamics under physiological conditions and allowing arbitrarily fast-changing time-dependent inputs to be encoded undistorted (Köndgen et al. [100]).

Ensembles of LIF models were shown to act low-pass filter when treated with additive white noise (Brunel and Hakim [25]; Brunel et al. [23]). Adversely, temporally correlated noise as the input current triggers a response that remains finite even for high frequencies (Brunel et al. [23];Fourcaud and Brunel [61]). These properties of LIF was, however, an oversimplification, while the realistic neuron models exhibit same characteristics of response without a dependence on time correlation of noise. The main parameter for the cutoff frequency seems to be the onset rapidness (Fourcaud-Trocme et al. [63]; Naundorf et al. [130]).

The findings of Köndgen et al. [100] can be reproduced using a nonlinear integrateand-fire (NLIF) model with very small $\Delta_{T}$ and a nonlinearity which is intermediate between exponential and quadratic. The studies on NLIF and conductance-based models further predicted that the linear response of a neuron is always dominated by a low-pass filter behavior (Fourcaud-Trocme et al. [63]; Fourcaud-Trocme and Brunel [62]; Naundorf et al. [130]). 


\section{4 Imitation of Reality : Neuron Models}

The recognized physicist Yakov Frenkel once mentioned that a theoretical model of a complex system should be like a good caricature. He meant that an ideal model should emphasize the features that are most important and should downplay the details that are inessential. However, as he also mentioned, the dilemma about this argument is that a researcher is almost always unacquainted on which feature is essential and which is not until the phenomenon that is under study is understood well enough.

The single-neuron modeling would be a fine example for this dilemma. One must consider the details such as the relevant ionic conductances, neuron morphology and realistic input properties when modeling a particular aspect of its individual dynamics. What is more, one must also present predictions about the possible contributions of these dynamics to the information processing of the neuron in its physiological environment. However, such details are often unknown until they are explored in a theoretical or experimental framework. Also, only the understanding of how a single neuron operates as a part of a network reveals the facts about its coding and the level of details required for its modeling. To overcome the problem, associating the results of theoretical and experimental studies, even from different disciplines, can be more helpful than it is anticipated.

\subsection{Levels of Modeling}

The earliest Integrate-and-Fire (IF) model was proposed more than a hundred years ago (Lapicque, 1907). The model generates an AP when the integrated sensory or synaptic inputs to the model reach a threshold value. It still remains as a commonly referred model as it provides a simple mechanistic explanation for basic neural operations, e.g. encoding of stimulus amplitude in spike frequency (Herz et al. [79]). How the single neuron properties contribute to (in micro-scale) information processing and (in macro-scale) behavior is an intriguing question that directed scientists' interest 


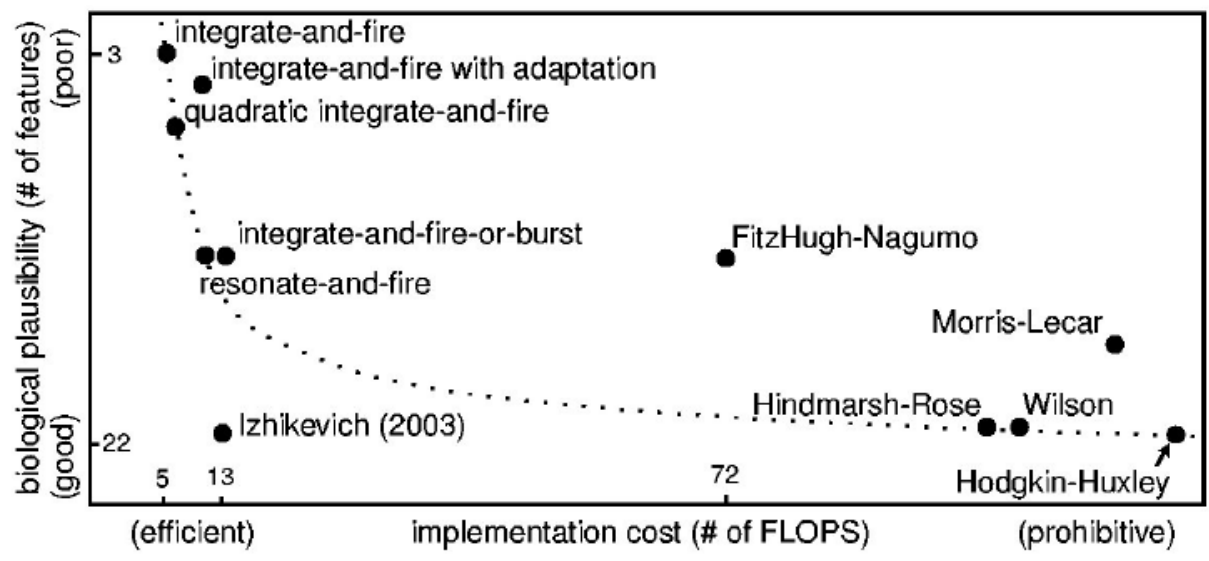

Figure 4.1: Model Comparison. The effiency of a model is defined under the purpose of the theoretical framework that it will be used in. Therefore, some more realistic and computationally expensive methods can be chosen over more simple methods. Figure adapted from Izhikevich [91].

further into theoretical studies. The neuron models can serve for a variety of purposes if only they can be fine-tuned to meet the respective needs of the study. Therefore, there must be a balance between implementing sufficiently detailed mechanisms that can account for the complex single neuron dynamics and reducing the complexity to only essential details to make the model tractable. Herz et al. [79] employed a classification of models according to their levels of complexity and mentioned the respective functional implications and weaknesses of each level (Figure 4.2). In my study, I will utilize a multi-compartmental model, which would stand somewhere between level I and II , and a single-compartment model as would be in level III.

\subsection{Realistic Approach}

The models with morphologically realistic geometry are based on the experimental findings. The anatomical reconstruction of a real neuron as a model provides information on the contribution of spatial structure of a neuron to its functions. The Rall model and the cable theory (Rall [144]) provided a basis for the more extended multi-compartmental models. Rall [147] showed mathematically that the dendritic voltage attenuation spreads asymmetrically. When voltage-dependent ionic conductances are also considered, the need for the multi-compartmental models emerge as the numerical integration over the spatially discretized dendrites will be required to solve the high-dimensional system of equations (Rall [145]). These models can be quite complicated when modeling complex structures. For instance, more than 1000 

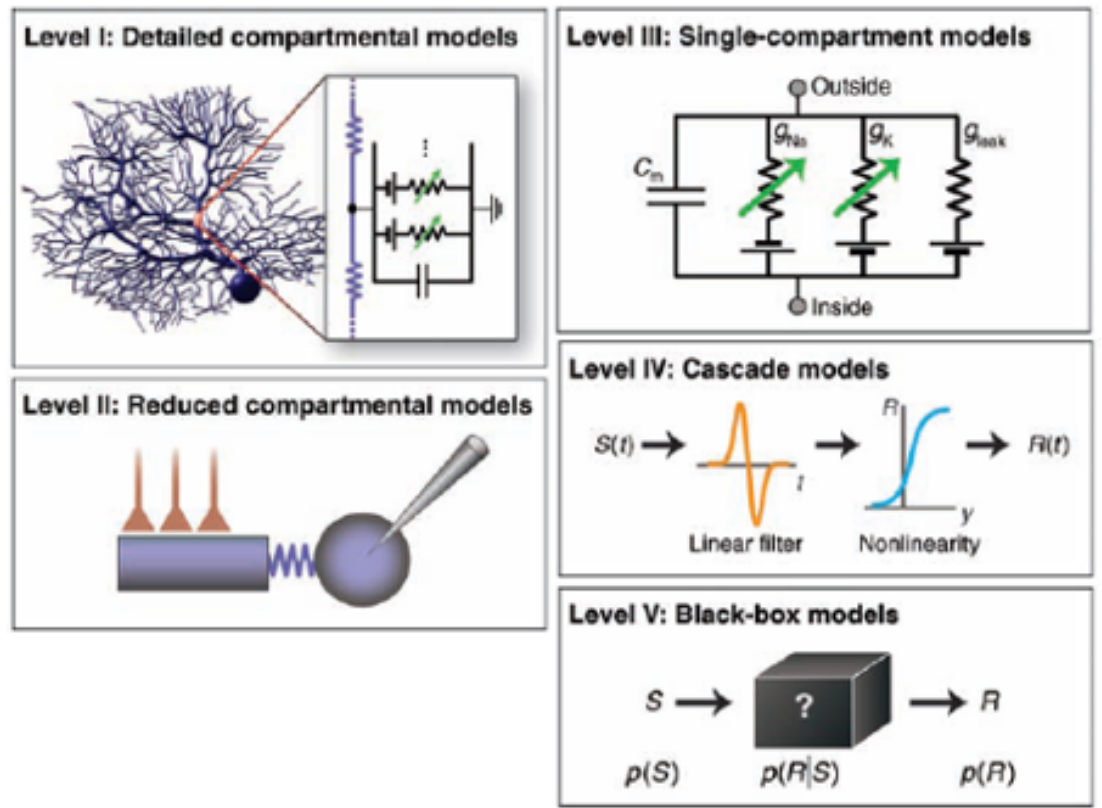

Figure 4.2: Examples of five levels of modeling. Level I: Detailed compartmental model of a Purkinje cell. The dendritic tree is segmented into electrically coupled Hodgkin-Huxley-type compartments (level III). Level II: Two-compartment model as mentioned in the text. The dendrite receives synaptic inputs and is coupled to the soma where the neuron's response is generated. Level III: Hodgkin-Huxley model, the prototype of single-compartment models- the cell's inside and outside are separated by a capacitance $\mathrm{C}_{m}$ and ionic conductances in series with batteries describing ionic reversal potentials. $\mathrm{Na}^{+}$and $\mathrm{K}^{+}$potassium conductances $\left(\mathrm{g}_{N a}, \mathrm{~g}_{K}\right)$ depend on voltage, the leak $\mathrm{g}_{\text {leak }}$ is fixed. Level $I V$ : Linear-nonlinear cascade. Stimuli $\mathrm{S}(\mathrm{t})$ are convolved with a filter and then fed through a nonlinearity to generate responses $\mathrm{R}(\mathrm{t})$, typically time-dependent firing rates. Level $V$ : Black-box model. Neglecting biophysical mechanisms, conditional probabilities $\mathrm{p}(\mathrm{R} \mid \mathrm{S})$ describe responses $\mathrm{R}$ for given stimuli S. Adapted from Herz et al. [79]. 


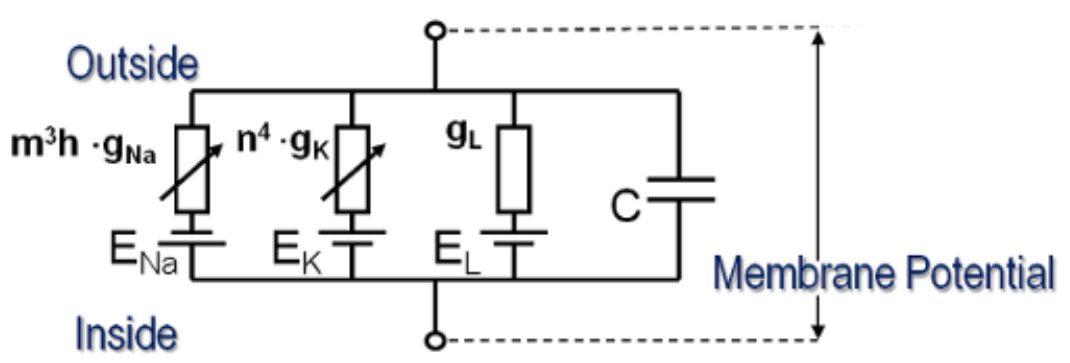

Figure 4.3: A general scheme of a conductance-based (CB) model. The model includes only $\mathrm{Na}^{+}, \mathrm{K}^{+}$and leak currents, as also in Wang-Bużsaki (WB) model.

compartments are required to model the dendritic tree of a pyramidal neuron when simulating the spike back-propagation (Golding et al. [67]).

Obviously, the high dimensionality and elaborated geometry of the models mentioned above are serious drawbacks as their emergent properties are not completely mathematically tractable. However, more reduced and still multi-compartmental models can also serve as realistic models. For instance, two-compartment models were used to indicate that homeostatic plasticity can follow from cellular learning rules to recalibrate the channel densities on the dendrites (Stemmler and Koch [170]) and to optimize the spike encoding of synaptic inputs.

The more complicated models also often include the equations for gating kinetics of ionic channels. The types of ionic conductances vary for almost each CB model. The general expression for a CB model can be given as

$$
C_{m} \dot{V}(t)=-i_{m}+I_{i n j} / A
$$

where $C_{m}$ is the membrane capacitance, $i_{m}$ is the membrane current that includes various ionic conductances, $I_{i n j} / A$ is the injected current per surface area.

To produce a full AP waveform, at least one $\mathrm{Na}^{+}$and one $\mathrm{K}^{+}$conductances are required. Therefore, I used a model with instantaneous $\mathrm{Na}^{+}$current, a $\mathrm{K}^{+}$current and leak current by modifying the model given by Wang and Buzsàki [183] (Figure 4.3). 


\subsection{Simplistic Approach}

The demanding and time-consuming nature of realistic but complicated neuron models led to a search for simpler models that can still account for the neuronal dynamics. As the first simplification, the spatial structure of the neuron is discarded and the ionic conductances and their contribution to the AP generation and subthreshold response behavior are targeted instead. The models, such as the classic single-compartment Hodgkin-Huxley (HH) model ([83]), are still useful in understanding, e.g., the firing pattern and spike frequency adaptation. However, it was also shown that some classical assumptions and utilization of $\mathrm{HH}$ model should be reconsidered as they can not fully reproduce the onset dynamics of cortical neurons (Naundorf et al. [132]).

\subsubsection{IF and Other Idealized Models}

Although, the embodiment of the ionic conductances into the model is crucial when the biophysical properties of a single neuron is investigated, the modeling on a more conceptual level allows direct addressing to its computation. Therefore, linearnonlinear cascade systems and black-box approach are other simplified yet useful tools (Herz et al. [79]). Also, further mathematical reductions and bifurcation analysis of HH models (Rinzel and Ermentrout [153]; Izhikevich [91]) provided insight for the observed response properties of IF and also resonate-and-fire (RF) neuron models.

The general form of IF models is

$$
\dot{C(V)}=-g_{L}\left(V-E_{L}\right)+\Psi(V)+I_{s y n}(t)
$$

where $C$ is the membrane capacitance, $g_{L}$ is the leak conductance, $E_{L}$ is the reversal potential for leak conductance and $I_{\text {syn }}(\mathrm{t})$ is the synaptic current. The $\Psi(\mathrm{V})$ component determined the characteristics of the IF model. The simplest form of IF models, leaky IF (LIF), takes $\Psi(\mathrm{V})=0$. LIF was used to study the linear response properties in the following years; however, it was also shown that this model does fully reproduce the response of neither the more complicated conductance-based (CB) models nor the real neurons (Fourcaud-Trocme et al. [63]; Brunel et al. [23]; Fourcaud-Trocme and Brunel [62]; Naundorf et al. [130], [131], [132]). Therefore, instead of this model, other extended yet simple models were proposed. For instance:

- LIF models can be expanded to include a threshold potential where $\Psi^{\prime}\left(V_{T}\right)=g_{L}$; a constant, $\Delta_{T}=g_{L} / \Psi^{\prime \prime}(V)$, that is in inverse relation with onset slope and the threshold current for tonic firing, $I_{T}=g_{L}\left(V_{T}-V_{L}\right)-\Psi\left(V_{T}\right)$ as in exponential IF $(\mathrm{EIF})$,

$$
\Psi(V)=g_{L} \Delta_{T} \exp \left(\frac{V-V_{T}}{\Delta_{T}}\right)
$$




\begin{tabular}{|c|c|c|}
\hline MODEL & METHOD & AUTHOR(S) \\
\hline LIF & (a) additive white noise & $\begin{array}{c}\text { Tuckwell [178]; } \\
\text { Brunel and Hakim [25]; } \\
\text { Brunel [22]; } \\
\text { Lindner and Schimansky-Geier [110] }\end{array}$ \\
\hline & (b) multiplicative white noise & Tuckwell [178], Richardson [150] \\
\hline & (c) colored noise, short $\tau_{I}$ limit & $\begin{array}{c}\text { Brunel and Sergi [27]; } \\
\text { Brunel et al. [23]; } \\
\text { Fourcaud and Brunel [61] }\end{array}$ \\
\hline & (d) colored noise, long $\tau_{I}$ limit & Moreno-Bote and Parga [128] \\
\hline RF & $\begin{array}{l}\text { (e) low sub-subthreshold } \\
\text { frequency limit }\end{array}$ & $\begin{array}{c}\text { Richardson et al. [151]; } \\
\text { Brunel et al. [24] }\end{array}$ \\
\hline QIF & & $\begin{array}{c}\text { Lindner et al. [109]; } \\
\text { Brunel and Latham [26] }\end{array}$ \\
\hline EIF & & $\begin{array}{c}\text { Fourcaud-Trocme et al. [63]; } \\
\text { Fourcaud-Trocme and Brunel [62] }\end{array}$ \\
\hline
\end{tabular}

Table 4.1: A general overview for integrate-and-fire models in the literature.

or in quadratical IF (QIF)

$$
\Psi(V)=\frac{g_{L}}{2 \Delta_{T}}\left(V-V_{T}\right)^{2}+g_{L}\left(V-V_{L}\right)-I_{T} \exp \left(\frac{V-V_{T}}{\Delta_{T}}\right)
$$

Fourcaud-Trocme et al. [63]; Fourcaud-Trocme and Brunel [62]) or they can be expanded to generate more nonlinear characteristics as in nonlinear IF (NLIF) (Fourcaud-Trocme and Brunel [62]).

- Naundorf et al. [131] utilized the theta-neuron ( $\theta$-neuron) with a technique that allows to compute the response at arbitrary frequencies.

- Another simple yet very efficient model to study the relationship between input statistics and the neuronal output is the threshold model (Burak et al. [28]; Jung [93]; Naundorf [129]). This model was also further developed as the Gaussian neuron model in a study by Tchumatchenko et al. [176].

In my studies, I will adapt the threshold model to use on a more complicated multi-compartmental model in Part II. I will also compare my results on dynamic linear response in CB models with IF models in discussion of Part II and Part III. 


\section{Part II}

\section{The effect of threshold variability on somatic spike statistics in multi-compartmental models}





\section{Abstract}

In most of the studies dealing with action potential initiation, electrophysiological recordings on neurons are conducted at the soma instead of the actual initiation site. The reason behind this design is the technical difficulties of recording from thin extension like axon. The actual action potential (AP) initiation site in cortical neurons is the axon initial segment (AIS), which is 30-100 $\mu \mathrm{m}$ away from the soma. Therefore, it is a topic of current interest whether somatic recordings faithfully reflect the properties of AP initiation at AIS initiation site. In this study, a multi-compartmental conductance-based model, which is a modified version of Rall's Ball-and-stick model (Rall [144]), was used together with a statistical framework to analyze the somatic potential fluctuations at a threshold-crossing time point in an axonal compartment. This study provides information about whether the onset potential for spike generation in soma and AIS are identical or can strongly differ. 


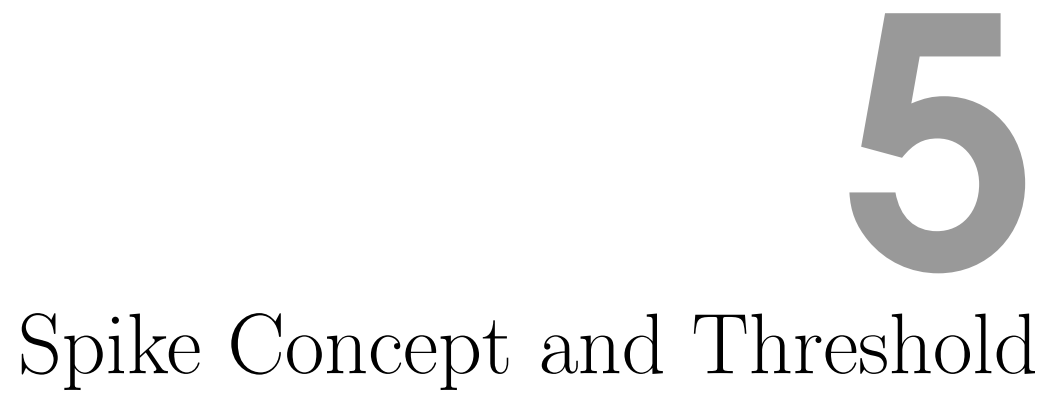

The studies on the information encoding in neural networks or in a single neuron will need complicated models when they are focused on the spike ${ }^{1}$ generating mechanisms (Meeks and Mennerick [125]; Mainen et al. [117]; Kole et al. [97]). However, topics such as relating the neuronal response to the input characteristics do not demand such complexity. Even in some of the very early studies, simpler models of firing behavior of neurons were described, for instance, by using a binary sequence of silent and firing states (McCulloch and Pitts [124]). A recent simplistic threshold model is proved to be sufficient to study the correlations between the input and the neuronal response (Tchumatchenko et al. [176]). Still the response given by a threshold model strongly depends on the varying threshold. Therefore, we used a slightly more complex model (Rall [147]) to explore the effect of the threshold variability (or the choice of threshold) on spike statistics and dynamic response properties.

The spike statistics are carried on using Spike-Triggered Average (STA) and Spike-Triggered Variance (STV). Our results suggest that STA is linearly dependent on the threshold and increases by distance from somatic end. On the other hand, STV is not dependent on threshold, or STA.

\subsection{Concept of Threshold}

A neuron spikes only when it is sufficiently excited to cross a certain potential, namely the voltage threshold, and no spikes can occur below this threshold.

The criterion to determine the threshold is usually defined to fulfill the particular needs of individual experimental designs. For instance in in vitro studies, the threshold might be defined over a certain parameter in the stimulus. This excitability

\footnotetext{
${ }^{1}$ To avoid confusion with the definitions that follows in this chapter, the term spike will be used instead of action potential. A spike might differ in definition with action potential, when there is not an actual waveform (section 5.1).
} 
threshold represents the minimal values of the chosen parameter, which is needed to trigger a spike. Then, the membrane potential that corresponds to this excitability threshold will be taken as the voltage threshold. However, it is obviously impossible to choose such a stimulus parameter in an in vivo study, as the input to the neuron can not be controlled. In these cases, the voltage threshold is defined at the value at the onset of a spike (Platkiewicz and Brette [141]).

\subsection{Spike-Triggered Statistics}

When $\mathrm{V}_{x}\left(\mathrm{t}_{i}\right)>\Psi$, which means that there is a spike at location $x$ and time $t$, the somatic potential $\mathrm{V}_{s}$ at spike time $t$ can be stored and averaged over total number of threshold-crossings, $n$, to get what is called as the somatic Spike-Triggered Average (STA), and the variance can be estimated to get the somatic Spike-Triggered Variance (STV).

$$
\begin{gathered}
S T A=\frac{1}{n} \sum_{i=1}^{n} V_{s}\left(t_{i}\right) \\
S T V=\frac{1}{n-1} \sum_{i=1}^{n}\left(V_{s}\left(t_{i}\right)-S T A\right)^{2}
\end{gathered}
$$

For a comparison to the numerical results, the analytical STA and STV were calculated with the following assumptions (Wei et al.- submitted) :

- The soma is a point $(\mathrm{x}=0)$ and the axon is long-enough to treat as a semi-infinite cable.

- The membrane potential fluctuations at the soma can be represented by Gaussian noise.

Therefore, the model soma acts as a simple low-pass filter and

$$
\begin{gathered}
S T A=\left(\sqrt{\frac{\pi}{2}} \sigma_{\dot{V_{x}}}^{-1} C_{V_{s} \dot{V}_{x}}^{2}(0)\right)+\Psi \sigma_{V_{x}}^{-2} C_{V_{s} V_{x}}(0) \\
S T V=\sigma_{V}^{2}-\sigma_{V_{x}}^{-2} C_{V_{s} V_{x}}^{2}(0)-\left(\left(\frac{\pi}{2}-1\right) \sigma_{\dot{V}_{x}}^{-2} C_{V_{s} \dot{V}_{x}}^{2}(0)\right)
\end{gathered}
$$


where for a time lag $\tau$

$$
\begin{gathered}
C_{V_{s} V_{x}}(\tau)=\left\langle V_{s}(t) V_{x}(t+\tau)\right\rangle \\
C_{V_{s} \dot{V}_{x}}(\tau)=\left\langle V_{s}(t) \dot{V}_{x}(t+\tau)\right\rangle
\end{gathered}
$$
$\mathrm{V}_{s}$.

and $\sigma_{V_{x}}^{2}$ is the variance of $V_{x}, \sigma_{\dot{V}_{x}}^{2}$ is the variance of $\dot{V}_{x}$ and $\sigma_{V}^{2}$ is the variance of

More detailed calculations are given in chapter 6 . The near-threshold potential average and variance in the soma is used as a tool of somatic detection of the axonal action potential initiation. Therefore, when a spike was detected at time $t_{i}$ at a location $x$ on the axon, somatic STA and STV were estimated at $\mathrm{V}_{s}\left(t_{i}\right)$, meaning $\tau=0$. For this reason, the covariances $C_{V_{s} V_{x}}(0)$ and $C_{V_{s} \dot{V}_{x}}(0)$ were used instead of the correlation functions $C_{V_{s} V_{x}}(\tau)$ and $C_{V_{s} \dot{V}_{x}}(\tau)$ in equations 5.4 and 5.3. As given in equations 5.1 and 5.2, the numerical STA and STV were also estimated with $\tau=0$. An example for the numerical results of covariances $C_{V_{s} V_{x}}(0)$ and $C_{V_{s} \dot{V}_{x}}(0)$ is given in Figure 6.6.

From the analytical formulations, STV of the model is expected to be independent of the threshold whereas a linear dependence on the threshold is expected for STA. 


\section{6}

\section{Multi-compartmental (Ball-and-Stick) Threshold (BST) Model}

Spike-triggered statistics were obtained by using either passive (section 6.2) or partiallyactive (section 6.3.2) BST model as will be described in the following sections. In a passive threshold model, a spike is defined as the positive threshold crossing of $V(t)$ or $\Delta V(t)=V(t)-V_{\text {rest }}$ (Figure 6.1), where $V_{\text {rest }}$ is the resting potential. If the trace exhibits plateau-like formations above the threshold, $\Psi$, successive negative crossing of the threshold may also be included in the criteria to distinguish a spike. Therefore, these so-called spikes do not need to exhibit a full action potential waveform in our numerical simulations, which enables us to work with a passive model. During a trial, the threshold can remain constant throughout the neuron (constant threshold) or change as a function of time and/or distance (dynamic threshold). In this study, a constant threshold was used.

\subsection{Model Methods}

In BST model, the soma was not single-compartment, but was divided into 10 compartments with $1 \mu \mathrm{m}$ each. Therefore, the radius of the sphere-like soma was $5 \mu \mathrm{m}$ and the surface area of the soma was approximately $150 \mu \mathrm{m}^{2}$. Each somatic compartment was homogeneous in passive electrical properties and in channel densities. The axon had a radius of $r_{a}=0.5 \mu \mathrm{m}$. The first $10 \mu \mathrm{m}$ after the soma was divided into 10 compartments with $1 \mu \mathrm{m}$ each. After this point,each compartment had a length of $10 \mu \mathrm{m}$ and the overall $\mathrm{L} \cong 2000 \mu \mathrm{m}$. Therefore, the axon was long enough to treat as a semi-infinite cable. The axonal compartments were also homogeneous in passive electrical properties and in channel densities. $\lambda \cong 410 \mu \mathrm{m}$ and $\tau_{m}=10 \mathrm{~ms}$. 


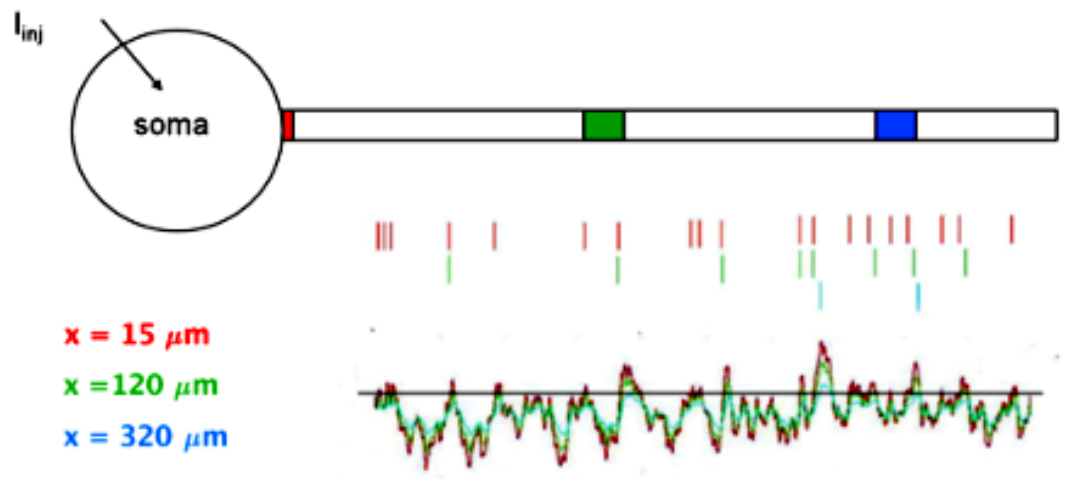

Figure 6.1: Passive BST Model: Upper figure gives a representation of the passive ball-and-stick model. On the model, the compartments at $x=15 \mu \mathrm{m}(\mathrm{red})$, $x=120 \mu \mathrm{m}$ (green) and $x=320 \mu \mathrm{m}$ (blue) were marked. In the lower figure, the subthreshold membrane potential traces of the marked compartments were given with corresponding colors. The solid black line represents the constant threshold, $\Psi=5$ $\mathrm{mV}$. The colored ticks above the traces represent the time points of positive threshold crossings, or spike times, for the trace with corresponding color.

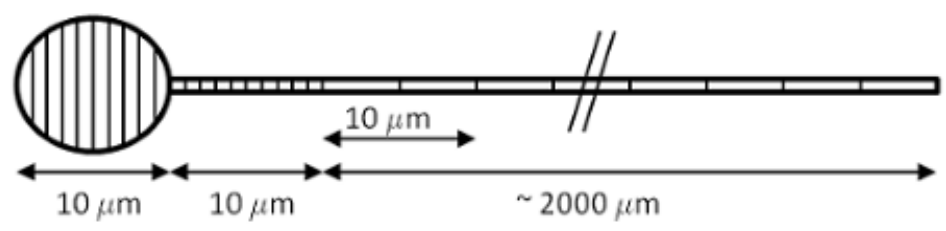

Figure 6.2: The ball-and-stick model was used as a multi-compartmental threshold model in our studies. The model had two parts: a sphere-like soma $(d=10 \mu \mathrm{m})$ and a cylindrical axon, that is thin $(d=1 \mu \mathrm{m})$ and long $(\mathrm{L} \cong 2000 \mu \mathrm{m} \cong 5 \lambda)$ enough to treat as an infinite cable. 


\subsubsection{The Cable Theory}

The probability that the neuron will fire an action potential or not strongly depends on the attenuation and the delay of electrical signals through the thin and long extensions of the neuron: dendrites and axon. These extensions can be treated as cable-like structures when investigating the response (i.e. subthreshold) dynamics of spatially extended neuron models. Therefore, the so-called cable theory provides a useful theoretical framework.

In the cable theory, the ion flux through and out the membrane is estimated first to construct the equation for the change in membrane potential in time $t$ and displacement $x$ domains.

Due to the voltage gradient over $x$, a longitudinal current $I_{l}$ flows within a neuron. For a section on the cylindrical extension that has a radius $a$ and a length $\Delta x$, the longitudinal resistance $R_{l}$ is estimated as :

$$
R_{l}=\frac{r_{L} \Delta x}{\pi a^{2}}
$$

where $r_{l}$ is the intracellular resistivity. Then, the longitudinal current $I_{l}$, that flows in the direction of $x$ will lead to a voltage drop of $\Delta V$, such that

$$
\Delta V=-I_{l} R_{l}=-I_{l} \frac{r_{l} \Delta x}{\pi a^{2}} .
$$

Thus, for any point along the cable, $I_{l}$ is given as

$$
I_{l}=-\frac{\pi a^{2}}{r_{l}} \frac{\partial V}{\partial x}
$$

To determine the membrane potential $V(x, t)$, the cable equation is derived as a partial differential equation using the currents that flow through and out a compartment of length $\Delta x$.

$$
c_{m} \frac{\partial V}{\partial t}=\frac{a}{2 r_{l}} \frac{\partial^{2} V}{\partial x^{2}}-i_{m}+i_{e}
$$

where $a$ is the radius of the cable and independent of $x$, and $i_{e}$ is the external current. The equation itself implies certain boundary conditions. For instance (Dayan and Abbott [47]):

- At the branching nodes, $V(x, t)$ must be continuous, which means that the functions $V(x, t)$ defined along each of the segments must give the same results at the node $x$. Also, at a node, the sum of entering or leaving longitudinal currents must be zero. 
- Another boundary condition is imposed at the end of the cable : No current should flow out of the end. This means that the spatial derivative must vanish at a terminal point.

A more detailed mathematical analysis of the Ball-and-Stick model and the boundary conditions is given in Appendix A.

\subsubsection{Application of Cable Equation to the Ball-and-Stick Model}

A model that is a highly simplified, yet still captures some of the important features of passive neuronal properties, was presented by Rall [144],[146]. In this model, a single-compartment, compact soma region is connected to a single equivalent cylindrical cable. In the original model, this extension represents the entire dendritic region of the neuron. The effect of critical passive neuron parameters, such as membrane time constant and electrotonic length constant, on the unique neuronal response can be analyzed using this simplistic model.

The cable equation for semi-infinite and non-branching cable-like extensions can be adapted by the simple ball-and-stick (BS) model, where the model has only passive conductance and the same passive electrical parameters for both soma and axon. Multiplying the equation 6.4 with $r_{m}$ (the membrane resistance), another representation of cable equation that uses the membrane time constant $\left(\tau_{m}=r_{m} . c_{m}\right)$ and the electrotonic length constant $\left(\lambda=\sqrt{\frac{a r_{m}}{2 r_{l}}}\right)$ as constant coefficients :

$$
\tau_{m} \frac{\partial V(x, t)}{\partial t}=\lambda^{2} \frac{\partial^{2} V(x, t)}{\partial x^{2}}-i_{m}(t) r_{m}+i_{e}(t) r_{m} .
$$

where $i_{m} . r_{m}$ gives $V(x, t)$.

The subthreshold dynamics at soma can be represented as

$$
\begin{aligned}
\tau_{m} \frac{d V_{s}(t)}{d t} & =-V_{s}(t)+i_{e} r_{m} \\
\tau_{m} \frac{d V_{s}(t)}{d t} & =-V_{s}(t)+r_{m} i_{l_{0}}+r_{m} i_{i n}
\end{aligned}
$$

where

$$
\begin{gathered}
i_{l_{0}}=\frac{1}{A} I_{l}=-\left.\frac{\pi a^{2}}{2 r_{l}} \frac{\partial V(x, t)}{\partial x}\right|_{x=0}, \\
i_{\text {in }}=g_{L}^{2} \chi(t) .
\end{gathered}
$$


$\chi(\mathrm{t})$ is a Gaussian random input current in units of $\mathrm{mV}$. Also, $r_{m}=1 / g_{L}$.

Thus, after the substitutions, the equation 6.6 becomes

$$
\tau_{m} \frac{d V_{s}(t)}{d t}=-V_{s}(t)+\left.\frac{\pi a^{2}}{2 A r_{l} g_{L}} \frac{\partial V(x, t)}{\partial x}\right|_{x=0}+g_{L} \chi(t) .
$$

Here, by defining a dimensionless constant

$$
Z=\frac{\pi a^{2}}{2 A r_{l} g_{L} \lambda}
$$

and

$$
\mu(t)=g_{L} \chi(t)
$$

the equation 6.9 takes the final form of :

$$
\tau_{m} \frac{d V_{s}(t)}{d t}=-V_{s}(t)+\left.Z \lambda \frac{\partial V(x, t)}{\partial x}\right|_{x=0}+\mu(t)
$$

Treating the axon as a semi-infinite cable (as in BST model), Wei [184] calculated the transfer of voltage fluctuations in the axon using Green's function method. Then, in the framework of Gaussian neuron model (Tchumatchenko et al. [176]), he calculated analytically STA at soma. Here, I used his analytical framework as a comparison to numerical results I obtained from BST model. For this purpose, I briefly mention here the calculation of $V_{s}(t)$ and its dependence on current $\mu(t)$. The analytical formulations of STA and STV are as given in equations 5.3 and 5.4. The detailed explanations and derivations are given in Wei [184].

The same approach can be used when the BS model is not passive but has at least one compartment with active conductances (see section 6.3.2). However, this type of BS model can not be represented using a one-dimensional cable equation as there will be at least two distinct parts with different longitudinal resistances. Still, it is possible to adapt a two-dimensional cable equation for such systems Wei [184].

\section{The $V_{s}(t)-\mu(t)$ Relation}

To have a better understanding of the somatic potential $V_{s}(t)$ as a function of input current $\mu(t)$, first, Wei [184] used the Laplace transform to cable equation on time domain as follows :

$$
\frac{\partial^{2} V(x, s)}{\partial x^{2}}-\left(1+\tau_{m} s\right) V(x, s)=0 .
$$

Assuming that voltage at infinity has a finite value and applying the Green's function,

$$
V(x, s) \equiv V_{s}(s) \tilde{G}_{1}(x, s)=V_{s}(s) e^{-\sqrt{\tau_{m} s+1} x / \lambda}
$$


where $\tilde{G}_{1}(x, s)$ is the Laplace transform of Green's function $G_{1}(x, t)$ in time domain.

Now, taking the Laplace transform of equation 6.12 over the time domain

$$
\tau_{m} s V_{s}(s)=-V_{s}(s)+\left.Z \lambda \frac{\partial V(x, s)}{\partial x}\right|_{x=0}+\mu(s)
$$

and substituting $V(x, s)$

$$
\begin{aligned}
\tau_{m} s V_{s}(s) & =-V_{s}(s)+\left.Z \lambda \frac{\partial}{\partial x}\left(V_{s}(s) e^{-\sqrt{\tau_{m} s+1} x / \lambda}\right)\right|_{x=0}+\mu(s) \\
& =-V_{s}(s)+Z \lambda\left(-\frac{\sqrt{\tau_{m} s+1}}{\lambda}\right) V_{s}(s)+\mu(s) \\
& =-V_{s}(s)-Z \sqrt{\tau_{m} s+1} V_{s}(s)+\mu(s) \\
& =-\left(1+Z \sqrt{\tau_{m} s+1}\right) V_{s}(s)+\mu(s) \\
\mu(s) & =\left(\tau_{m} s+1+Z \sqrt{\tau_{m} s+1}\right) V_{s}(s) \\
V(s) & =\frac{1}{\tau_{m} s+1+Z \sqrt{\tau_{m} s+1}} \mu(s)
\end{aligned}
$$

\subsubsection{Analysis of Gaussian Neuron Model}

A more simplified version of our threshold model was built by Tchumatchenko et al. [176]. Using the analytical analysis given in this study, the spike measure $(\mathrm{s}(\mathrm{t})$ ) of a threshold model, which takes a stationary continuous random Gaussian process as the membrane potential trace $\mathrm{V}(\mathrm{t})$, can be defined as:

$$
\left.s(t)=\sum_{i} \delta\left(t-t_{i}\right)=\delta\left(V(t)-\Psi_{0}\right) \theta(\dot{V}(t))|\dot{V}(t)|\right)
$$

Then, the stationary firing rate is estimated as:

$$
\nu_{0}=\langle s(t)\rangle=\frac{1}{2 \pi \tau_{s}} \exp \left(-\frac{\Psi_{0}^{2}}{2 \sigma_{V}^{2}}\right)
$$

where

$$
\tau_{s}=\frac{\sigma_{V}}{\sigma_{\dot{V}}}
$$

Two important conclusions can be drawn from this formula : 
1. The term $\Psi_{0} / \sigma_{V}$ determines the probability of threshold crossings. A decrease in $\Psi_{0} / \sigma_{V}$ will automatically lead to a decrease in $\nu_{0}$.

2. The faster fluctuations lead to a higher rate of threshold crossings. This is represented in the equation as an increase in $\nu_{0}$ as $\tau_{s}$ decrease.

Also, the equation implies that it can only be used in fluctuation driven, low firing rate regime, as the maximum value of $\nu_{0}$ is limited by the term $1 / 2 \pi \tau_{S}$.

The analytical work (Wei [184]) utilized the Gaussian neuron model as the theoretical framework. The theoretical results of the model was presented as the correlation function of the membrane potential $C(\tau)$ and its derivatives, therefore, the implicit form of $C(\tau)$ can be determined by choice. In the study by Wei [184], a difference of two exponentials was chosen as the correlation function of the framework, such that:

$$
\begin{aligned}
& C_{V_{s}}(\tau)=\left\langle V_{s}(t) V_{s}(t+\tau)\right\rangle \\
& C_{V_{s}}(\tau)=\frac{\sigma_{V}^{2}}{\tau_{D}-\tau_{R}}\left(\tau_{D} \exp \left(-\frac{|\tau|}{\tau_{D}}\right)-\tau_{R} \exp \left(-\frac{|\tau|}{\tau_{R}}\right)\right) .
\end{aligned}
$$

Then, STA and STV were estimated as given in equations 5.3 and 5.4.

\subsubsection{Numerical Methods}

The model was implemented using $\mathrm{C}++$ in Linux environment (see Figure 6.3 for a detailed scheme of the contents of the code). The implicit backward Euler integration method was used with a $10 \mu$ s time step. The membrane capacitance $\left(\mathrm{C}_{m}\right)$ was 0.1 $\mathrm{nF} / \mathrm{mm}^{2}$. The membrane resistance $\left(\mathrm{r}_{m}\right)$ was $1 \mathrm{M} \Omega . \mathrm{mm}^{2}$, longtidunal membrane resistance, $r_{l}$, was $1.5 \mathrm{M} \Omega . \mathrm{mm}$ and the membrane time constant was estimated as 10 ms.

\subsubsection{Designing the Input Current}

Depending to their functional roles, many neurons are intrinsically very noisy, therefore, exhibit spontaneous firing even in the absence of an external stimulus and the outgoing spike train was considered as a random process even in the presence of a stimulus. Both the stimulus and intrinsic noise trigger the spike trains in a cooperative fashion (Wiesenfeld and Moss [186]). Therefore, in a realistic simulation, the input must be presented with a noisy component in addition to the deterministic part.

1. $\mu(t)$ 


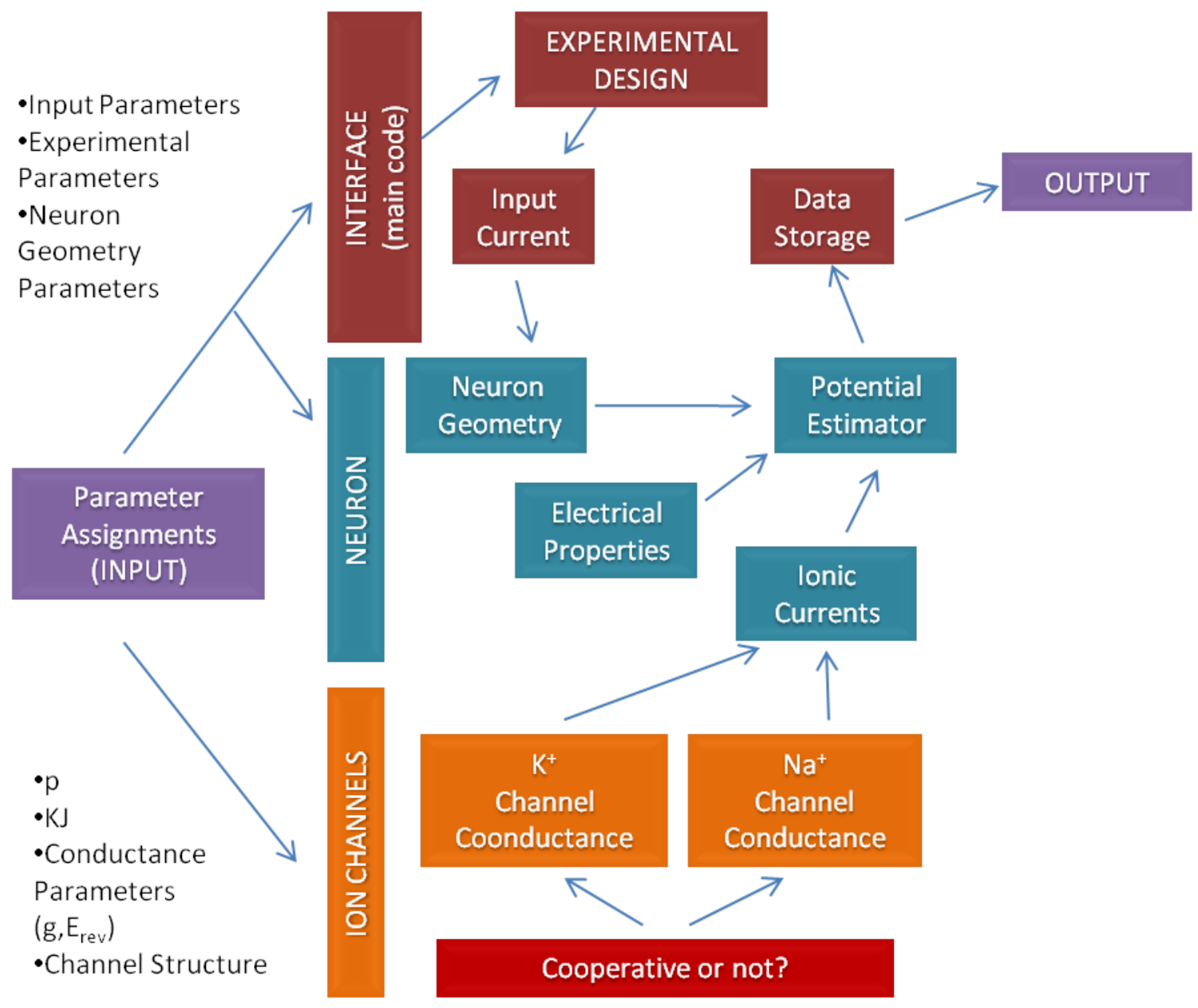

\section{MODELSTRUCTURE}

Figure 6.3: The contents of the model in $\mathbf{C}++$ code. The parameters $p$ and $K J$, the concept of cooperativity and the implementation of cooperativity in the model (red box) is explained in Part III. 
To obtain an analytical comparison to the numerical results of BST model, the analytical framework of Wei [184] was used as described in section 6.1.2 and 6.1.3. The equation 6.16 implies method of producing the input current to have the somatifc membrane potential $V_{s}(t)$ in a desired form. The desired $V_{s}(t)$ in our analytical and numerical frameworks was an Ornstein-Uhlenbeck process. Using the correlation functions for $V_{s}(t)$ and Fourier transforms, a current $\mu(t)$ was obtained, such that it has a variance $\sigma_{I}^{2}$ as

$$
\sigma_{I}^{2}=2\left(1+\frac{\tau_{R}}{\tau_{D}}\right) \sigma_{V}^{2} .
$$

The current was produced by a custom-written MATLAB procedure that uses Runger-Kutta integration method with a time step of $10 \mu \mathrm{s}$. Then, this current was fed into the source code of BST model.

\section{Ornstein-Uhlenbeck (OU) Process as Injected Current}

In the brain, neurons receive information under a bombardment of excitatory and inhibitory synaptic inputs. The activity of numerous synapses in the cortex creates a noisy background for the information processing of a single neuron. This background noise can be characterized by Gaussian statistics and by an autocorrelation function exponentially decaying with the time constant $\tau_{I}$.

As a part of my simulations, I used a noisy current $\left(I_{\text {noisy }}\right)$ that was generated as a realization of an Ornstein-Uhlenbeck stochastic process with zero mean and variance $\sigma_{I}^{2}$ to mimic the synaptic noisy input as following :

$$
\tau_{I} I_{n o i s y}^{\cdot}=-I_{n o i s y}+\kappa \zeta_{t}
$$

where $\zeta_{t}$ is a random variable drawn at every time step from a Gaussian distribution with a zero mean, called white noise; $\kappa=X / \Delta t$ and $\zeta_{t}=\xi_{t} / \Delta t$. Integrating the deterministic part gives us

$$
I_{\text {noisy }}(t+\Delta t)=I_{\text {noisy }}(t) \exp \left(-\frac{\Delta t}{\tau_{I}}\right)+X \xi_{t}
$$

and $\mathrm{X}$ can be set as

$$
X=\sigma_{I} \sqrt{1-\exp \left(\frac{2 \Delta t}{\tau_{I}}\right)}
$$

where $\sigma_{I}$ is the variance of $I_{\text {noisy }}$. 
The value of $\tau_{I}$ corresponds the decay time constants of synaptic currents. In a range of 5-20 ms fast synaptic currents such as AMPA- and GABA $_{A}$-mediated currents. The current was injected at the middle compartment of the soma (e.g. $5^{\text {th }}$ compartment).

\section{Sinusoidal Noisy Input Current}

To probe the dynamic response properties of the PA-BST model under in vivolike conditions, independent realizations of noisy current were synthesized in the source code of BST model and were injected at the soma .

$$
I_{i n j}=I_{n o i s y}+I_{\text {sin }}
$$

where $I_{n o i s y}$ is as in section 8.1.4 and

$$
I_{\sin }=I_{1} \sin \left(2 \pi f_{I} t\right)
$$

\subsubsection{Passive and Active Model Parameters}

The conductances of each compartment was adjusted as a modified version of WangBuzsaki model (Table 6.1). Each compartment had $\mathrm{K}^{+}$current, instantaneous $\mathrm{Na}^{+}$ current and leak current. If the model was set as passive, then all the active conductances $\left(\mathrm{Na}^{+}\right.$and $\left.\mathrm{K}^{+}\right)$are discarded and the membrane current is modeled only by leak current.

The conductance-based BST model is expressed using classical channel gating mechanisms as suggested by Hodgkin and Huxley [83]. The dynamical changes in the membrane potential, $\mathrm{V}(\mathrm{t})$, is determined by integrating the equation

$$
c_{m} \dot{V}(t)=-i_{m}+\frac{I_{i n j}}{A},
$$

where the membrane current $i_{m}$ is

$$
i_{m}=i_{N a}+i_{K}+i_{L}
$$

and $I_{i n j}$, the injected current, is divided by the surface area, $A$.

Using Ohm's Law and defining the channel conductance as the product of maximal conductance and the opening probability of individual channels, the ionic currents are estimated as

Instantaneous Sodium Current :

$$
i_{N a}(t)=\bar{g}_{N a} m_{\infty}^{3}(t) h(t)\left(V(t)-E_{N a}\right)
$$




\begin{tabular}{|c||c|c|}
\hline Channel Type & Channel Density $(\bar{g})\left(\mathrm{pS} / \mu m^{2}\right)$ & Reversal Potential $(\mathbf{m V})$ \\
\hline \hline $\mathrm{Na}^{+}$channel & 350 & 55 \\
\hline $\mathrm{K}^{+}$ & 150 & -90 \\
\hline Leak & 1 & -65 \\
\hline \hline
\end{tabular}

Table 6.1: The maximal conductance per unit surface (or channel density) and reversal potential in Wang-Buzsaki model (Wang and Buzsàki [183]).

Potassium Current :

$$
i_{K}(t)=\bar{g}_{K} n^{4}(t)\left(V(t)-E_{K}\right)
$$

Leak Current :

$$
i_{L}(t)=\bar{g}_{L}\left(V(t)-E_{L}\right)
$$

where $E_{N a}, E_{K}$ and $E_{L}$ are the reversal potentials; $\bar{g}_{N a}, \bar{g}_{K}$ and $\bar{g}_{L}$ are the maximal conductances (Table 6.1) and $m, n$ and $h$ are the gating particles.

\subsubsection{Data Analysis}

Data were obtained from the simulation as either potential traces, spike times or both. Data analysis were performed in MATLAB v.7.0.0 and v7.2.0 using custom-written functions and procedures.

The number of repetition for same set of current parameters $\left(\mu_{I}, \sigma_{I}, \tau_{I}, I_{1}\right.$ and $\left.f_{I}\right)$ were at least 3000. An example of the sinusoidally modulated response is given as raster plot of 1000 spike train repetitions in Figure 6.27. The explored range of frequency was $1-500 \mathrm{~Hz}$. The noisy current parameters were not varied. $I_{1}$ value was set to $10 \%$ of the $\mu_{I}$ value. The spikes were pooled with respect to the spike times and then, were binned into a Peristimulus Time Histogram (PSTH). The bin size was $1 / 30$ of $1 / f_{I}$, where $f_{I}$ is the frequency of the injected sinusoidally oscillating current. The spike count average over repetitions were fit using a least-squares method with the equation

$$
\nu(t)=\nu_{0}+\nu_{1} \sin \left(2 \pi f_{I} t+\phi\right) .
$$

Gain of the modulation was obtained as $\nu_{1} / \nu_{0}$ for frequency $f_{I} \cdot \nu_{0}$ was chosen as $\approx$ $10 \mathrm{~Hz}$. The confidence interval for $\alpha=0.05$ was given as error bars on $\nu_{1} / \nu_{0}-f_{I}$ curve.

To compare the analytical results with the numerical results, a two-way ANOVA test was used and the p-values were given in parenthesis in the text, where necessary. 


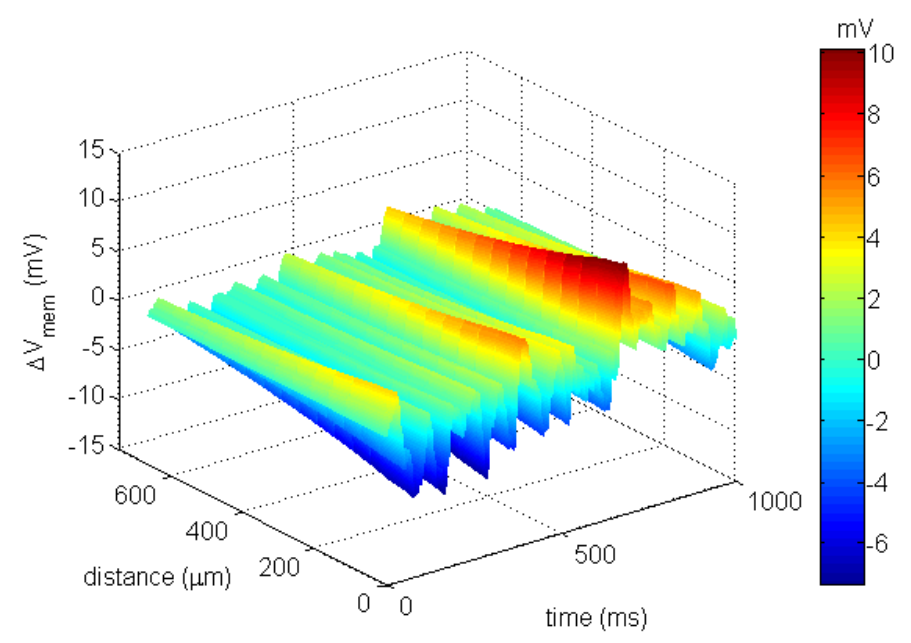

Figure 6.4: 3-D representation of subthreshold potential response $(\Delta \mathrm{V}=\mathrm{V}$ - $\mathrm{V}_{\text {rest }}$ ) in passive BST model. The response was obtained under noisy current injection $\left(\sigma_{I}=9 \mathrm{pA}, \tau_{I}=20 \mathrm{~ms}, \mu_{I}=0\right)$.

\subsection{Response Properties in Passive Model}

The subthreshold potential changes $\left(\Delta \mathrm{V}=\mathrm{V}-\mathrm{V}_{\text {rest }}\right)$ (Figure 6.4) as a response to the noisy current was treated with a constant threshold approach. The subthreshold response was expressed in a passive linear model, consisting of only the leak current :

$$
c_{m} \dot{V}=-g_{l}\left(E_{r e v}-V\right)+I_{i n j} .
$$

The attenuation in $\Delta \mathrm{V}$ became visible only approximately after $60 \mu \mathrm{m}$, with more attenuation at higher frequency fluctuations (Figure 6.5).

\subsubsection{Threshold Dependence of Spike Statistics}

Threshold variability in this model was computed as described in the previous sections by choosing threshold values on the axonal subthreshold fluctuations. The positive threshold crossings $\left(\Delta V_{x}\left(t_{i}\right)>\Psi\right)$ were considered as a spike. Then, the somatic STA and STV were estimated for $\Delta V_{s}\left(t_{i}\right)$.

A typical analysis set for the passive BST model under OU input current injection is given in Figure $6.7\left(\sigma_{I}=6 \mathrm{pA}, \tau_{I}=5 \mathrm{~ms}, \mu_{I}=0\right)$. At $\sigma_{I}=6 \mathrm{pA}, \sigma_{V} \cong 3.3 \mathrm{mV}$. The same data analysis was also performed for $\mu(t)$ as input current (see section 8.1.4). 


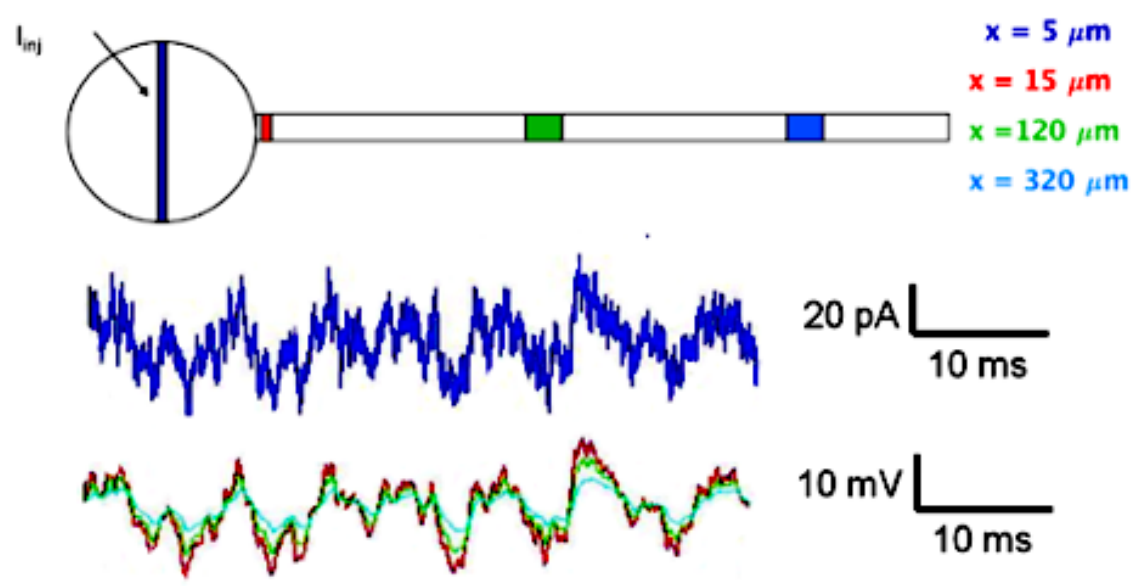

Figure 6.5: The current injection and the voltage response in passive BST model. The current is injected at $x=5 \mu \mathrm{m}$ in the soma and the voltage traces at different compartments are shown with the colors as given in the figure. Attenuation of the subthreshold fluctuation amplitude was visible at $x=120 \mu \mathrm{m}$ and $x=320 \mu \mathrm{m}$

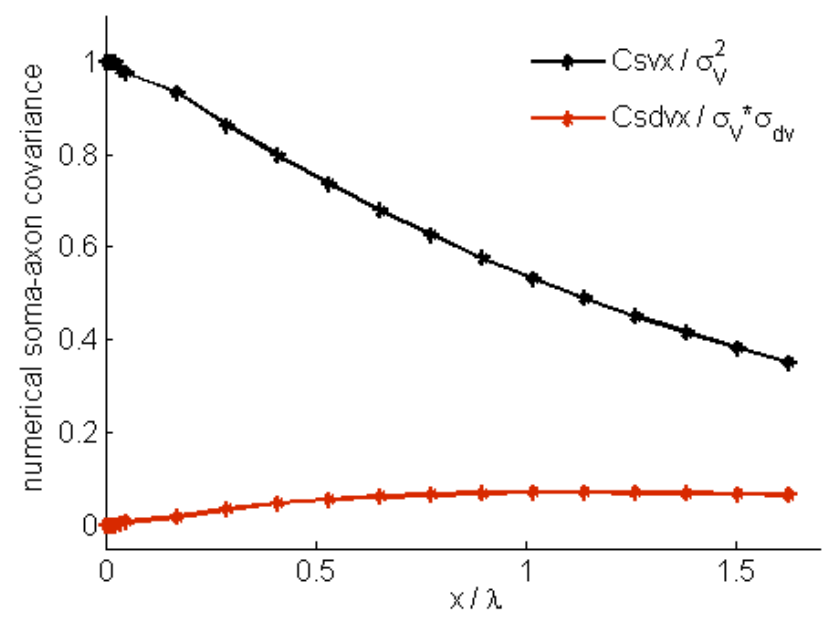

Figure 6.6: The normalized soma-axon correlation. The correlations $C_{V_{s} V_{x}}(0)$ and $C_{V_{s} \dot{V_{x}}}(0)$ were normalized as $C_{V_{s} V_{x}}(0) / \sigma_{V}^{2}$ and $C_{V_{s} \dot{V}_{x}}(0) / \sigma_{V} \sigma_{\dot{V}_{s}}$ and plotted against the distance in units of $\lambda\left(\operatorname{Csvx}=C_{V_{s} V_{x}} ; \operatorname{Csdvx}=C_{V_{s} \dot{V}_{x}} ; \sigma_{d v}=\sigma_{\dot{V}_{s}}\right)$. The statistics were obtained under noisy current injection $\left(\sigma_{I}=9 \mathrm{pA}, \tau_{I}=20 \mathrm{~ms}, \mu_{I}=0\right)$. 

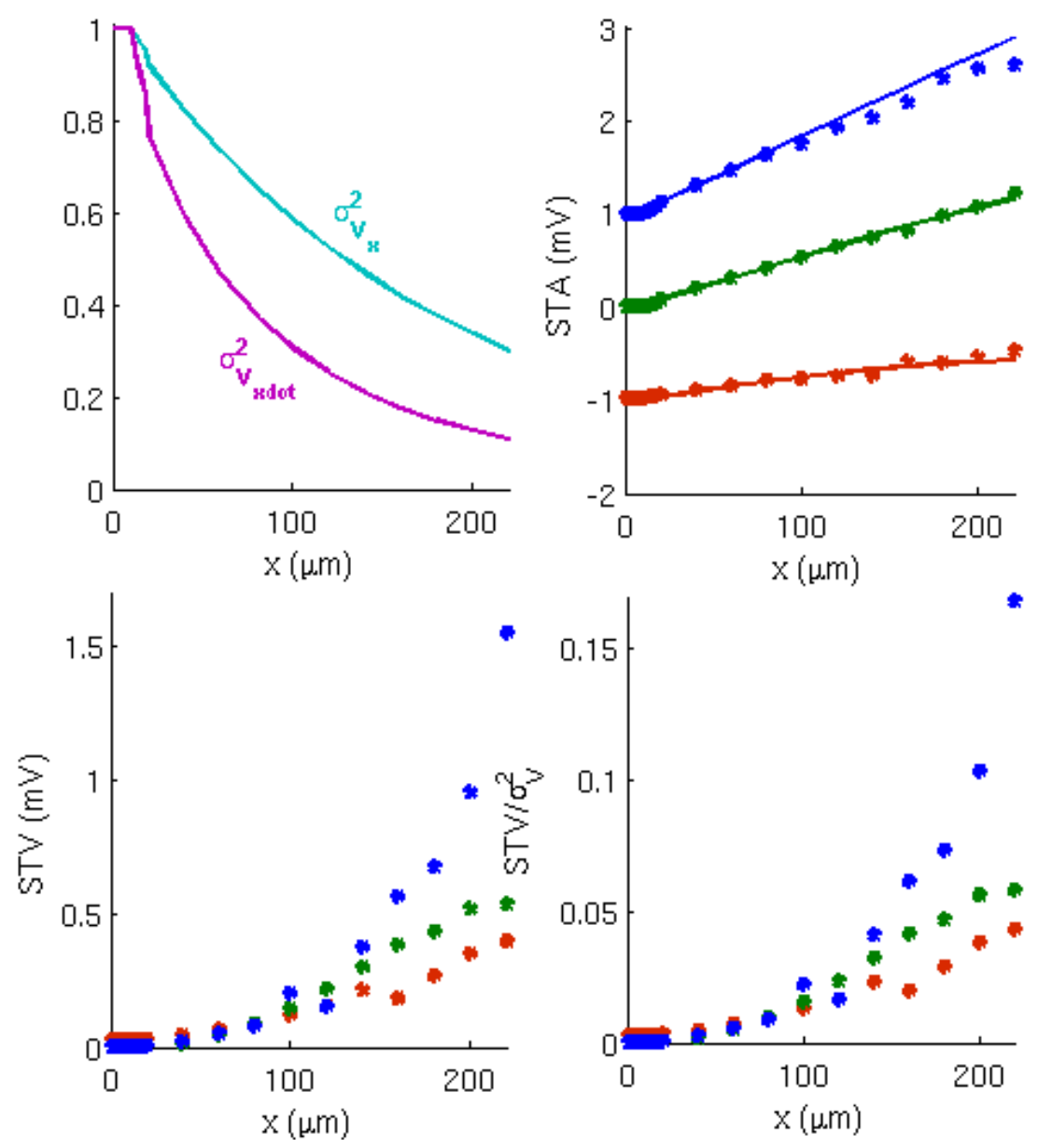

Figure 6.7: A typical analysis set for passive BST model. Upper left: The change in variance of the membrane potential at compartment $x, V_{x}$, and its derivative, $\dot{V}_{x}$ by increasing distance. Upper right: Numerical and analytical STA results for $\Psi=-1 \mathrm{mV}$ (red), $\Psi=0 \mathrm{mV}$ (green) and $\Psi=1 \mathrm{mV}$ (blue). Dots represent the numerical results whereas the solid lines represent the analytical results. Lower left: Numerical STV results for the same threshold values. Lower right: Numerical $\mathrm{STV} / \sigma_{V}$ results for the same threshold values. 


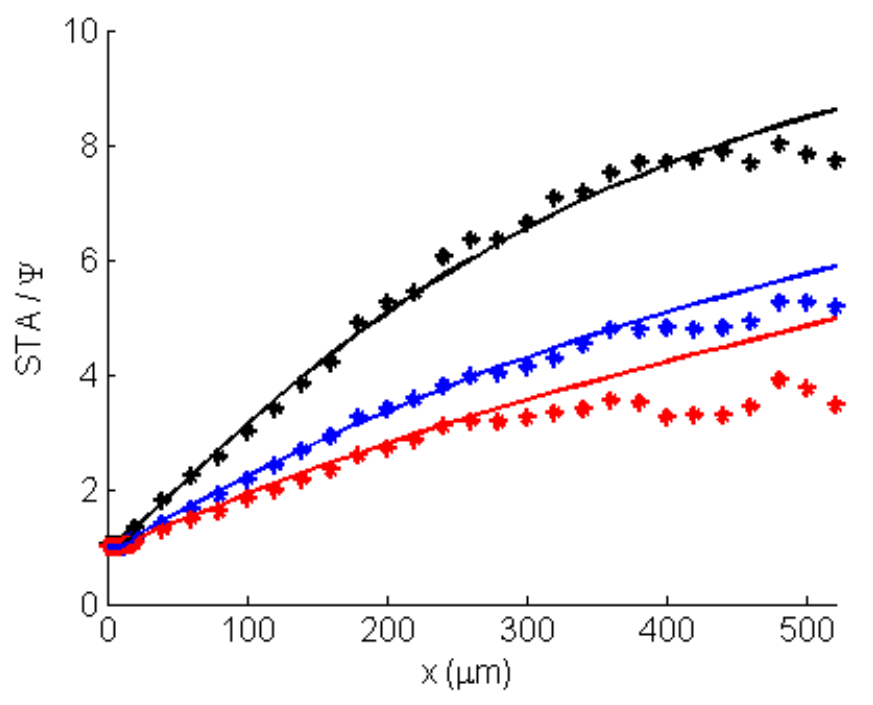

Figure 6.8: Dependence of STA on threshold variability and distance from somatic end in passive BST model under correlated current injection. $\mathrm{Nu}-$ merical and analytical results of STA/ $\Psi$ for threshold values $\Psi=1 \mathrm{mV}$ (black), $\Psi=$ $2 \mathrm{mV}$ (blue) and $\Psi=3 \mathrm{mV}$ (red). Solid lines represent the analytical results whereas the dots represent the numerical results. $\left(\tau_{R}=10, \tau_{D}=2\right)$.

STA results for both type of input currents the passive model were linearly dependent on the choice of $\Psi$ and on the distance from somatic end. For OU process as current, the analytical results were in match with the numerical results $(\mathrm{p}>0.05$; Figure 6.9). However, the results obtained by $\mu(t)$ as input current exhibited some differences. For instance, the linear dependence on distance almost disappeared after $x=400 \mu \mathrm{m}$. The analytical and numerical results also exhibited some discrepancy when $x>400 \mu \mathrm{m}$, but up to this point, they matched as in the previous case $(\mathrm{p}>0.05)$

On the other hand, STV was completely independent from the threshold values and was exponentially dependent on the distance from somatic end. STV exhibited slight dependence on threshold under $\mu(t)$ as input current. Until $200 \mu \mathrm{m}$ away from soma, the analytical and numerical results were coherent, however, after this point, they did not match $(\mathrm{p}<0.002)$.

\subsubsection{Impact of Current Statistics}

The current statistics, i.e. the variance, $\sigma_{I}$ and the time correlation, $\tau_{I}$, of the current affect the response properties of BST model. As $\sigma_{I}$ increases, the variance of the membrane potential, $\sigma_{V}$, is also expected to increase. As the analytical calcula- 


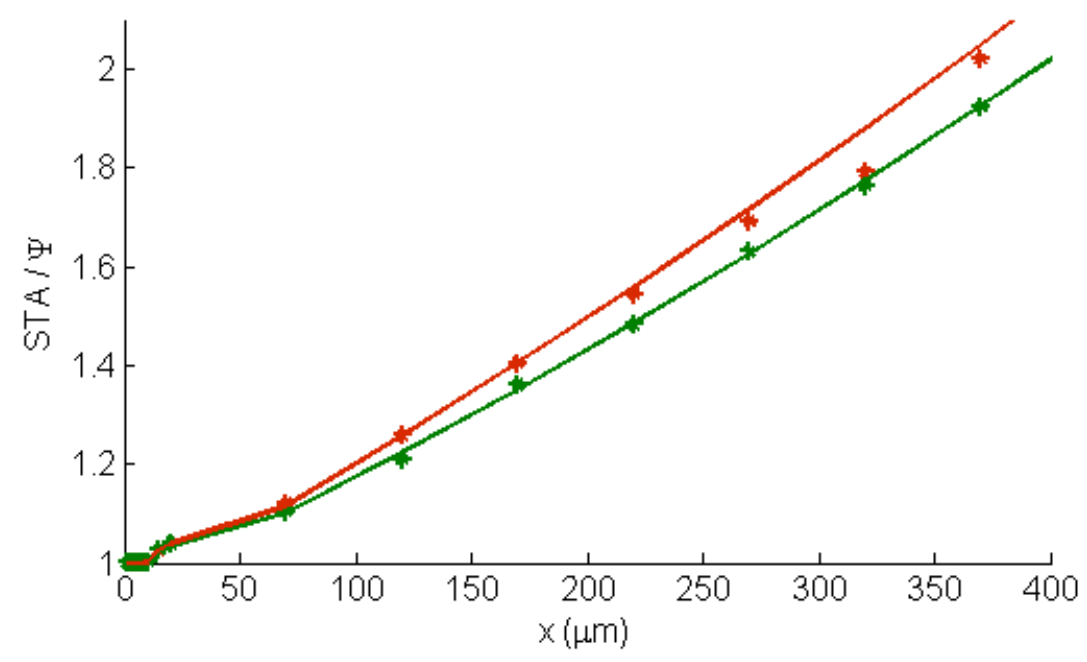

Figure 6.9: Dependence of STA on threshold variability and distance from somatic end in passive BST model under OU current injection. Numerical and analytical results of STA $\Psi$ for threshold values $\Psi=2 \mathrm{mV}$ (red) and $\Psi=3 \mathrm{mV}$ (green). Solid lines represent the analytical results whereas the dots represent the numerical results. $\left(\sigma_{I}=9 \mathrm{pA}, \tau_{I}=20 \mathrm{~ms}, \mu_{I}=0\right)$.

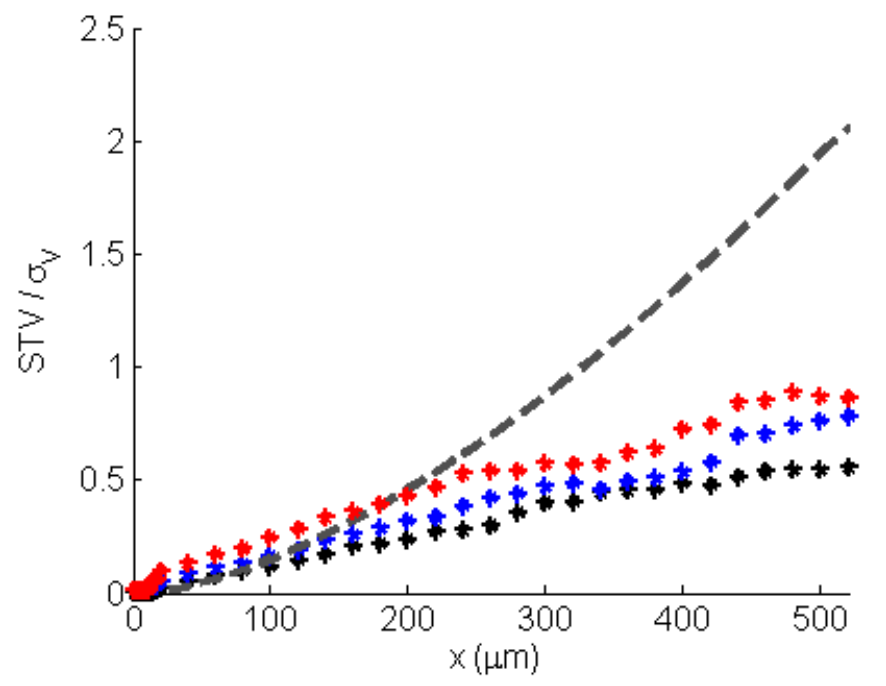

Figure 6.10: Dependence of STV on threshold variability and distance from somatic end in passive BST model under correlated current injection. $\mathrm{Nu}-$ merical and analytical results of STV $/ \sigma_{V}$ for threshold values $\Psi=2 \mathrm{mV}$ (red) and $\Psi=3 \mathrm{mV}$ (green). Dots represent the numerical results and the solid blue line represents the analytical result. $\left(\tau_{R}=10, \tau_{D}=2\right)$. 


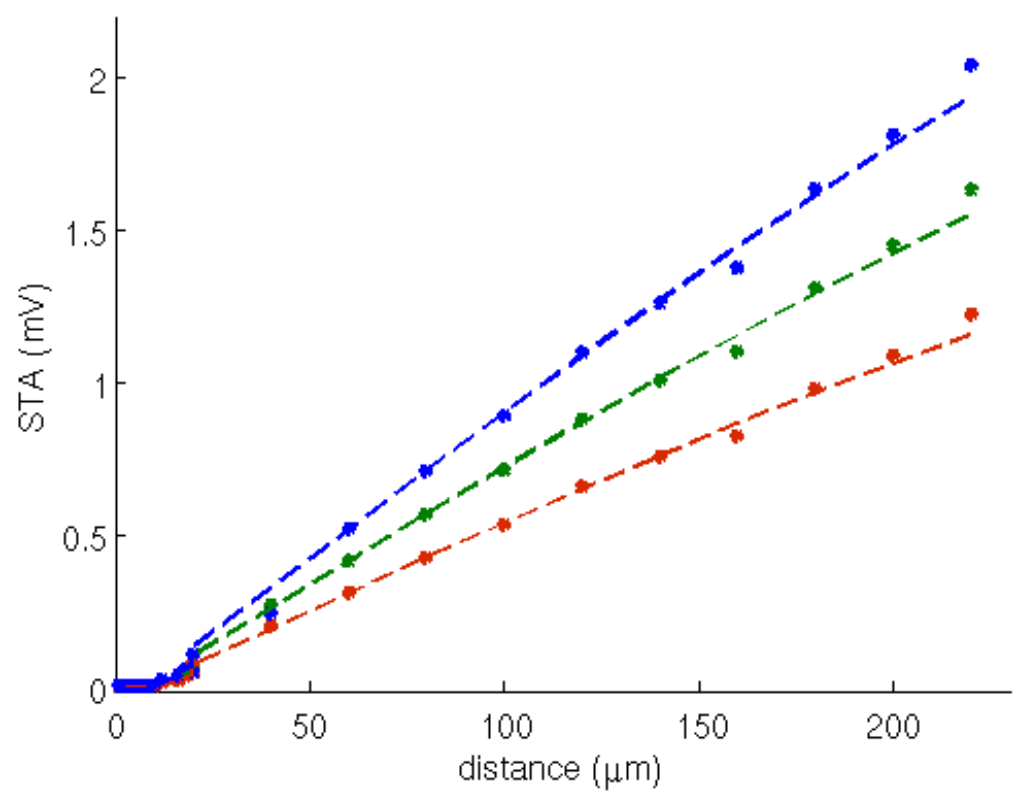

Figure 6.11: Dependence of STA on $\sigma_{I}$ values in passive BST model. $\Psi=$ $0 \mathrm{mV}$. Dashed lines represent the analytical results whereas the dots represent the numerical results. red: $\sigma_{I}=6 \mathrm{pA} ;$ green: $\sigma_{I}=8 \mathrm{pA} ;$ blue: $\sigma_{I}=10 \mathrm{pA}$.

tions of the STA and STV also point out, an increase in $\sigma_{V}$ will also lead to higher STA and STV values. Indeed, this phenomenon was observed both for numerical analytical results (Figure 6.11 and 6.12 ). Setting $\Psi=0, \tau_{I}=5 \mathrm{~ms}$ and $\mathrm{I}_{0}=0$, it was shown that the numerical and analytical STA increases more sharply by distance as $\sigma_{I}$ increases, and the numerical and analytical results still perfectly match for $\Psi$ $=0$ (The match when $\Psi>0$ was not as accurate as it is when $\Psi=0$.). On the other hand, the numerical and analytical values for STV also increases more sharply when $\sigma_{I}$ increases, but the discrepancy between the numerical and analytical STV also increases. This discrepancy is more pronounces after $75 \mu \mathrm{m}$. $\sigma_{v}$ values for $\sigma_{I}=$ $6 \mathrm{pA}, 8 \mathrm{pA}$ and $10 \mathrm{pA}$ were $3 \mathrm{mV}, 4 \mathrm{mV}$ and $5 \mathrm{mV}$ respectively.

The analytical relationship between $\tau_{I}$ and the statistics of the membrane potential was not as obvious as for $\sigma_{I}$. However, $\tau_{I}$ was expected to be inversely proportional to STV. $\sigma_{v}$ values for $\tau_{I}=5 \mathrm{~ms}, 10 \mathrm{~ms}$ and $20 \mathrm{~ms}$ were $3 \mathrm{mV}, 3.4 \mathrm{mV}$ and $3.7 \mathrm{mV}$ respectively. An increase in $\tau_{I}$ was expected to result in lower values of STA and $\mathrm{STV}$. Both the numerical and analytical results agreed with this prediction (Figure 6.13 and 6.14). For $\Psi=0, \sigma_{I}=6 \mathrm{pA}$ and $\mathrm{I}_{0}=0$, the increase in $\tau_{I}$ led to decrease in the STA and STV values, and therefore in the slopes of their relation with distance, $x$. Moreover, for larger $\tau_{I}$, the analytical and numerical STA results didn't match so 


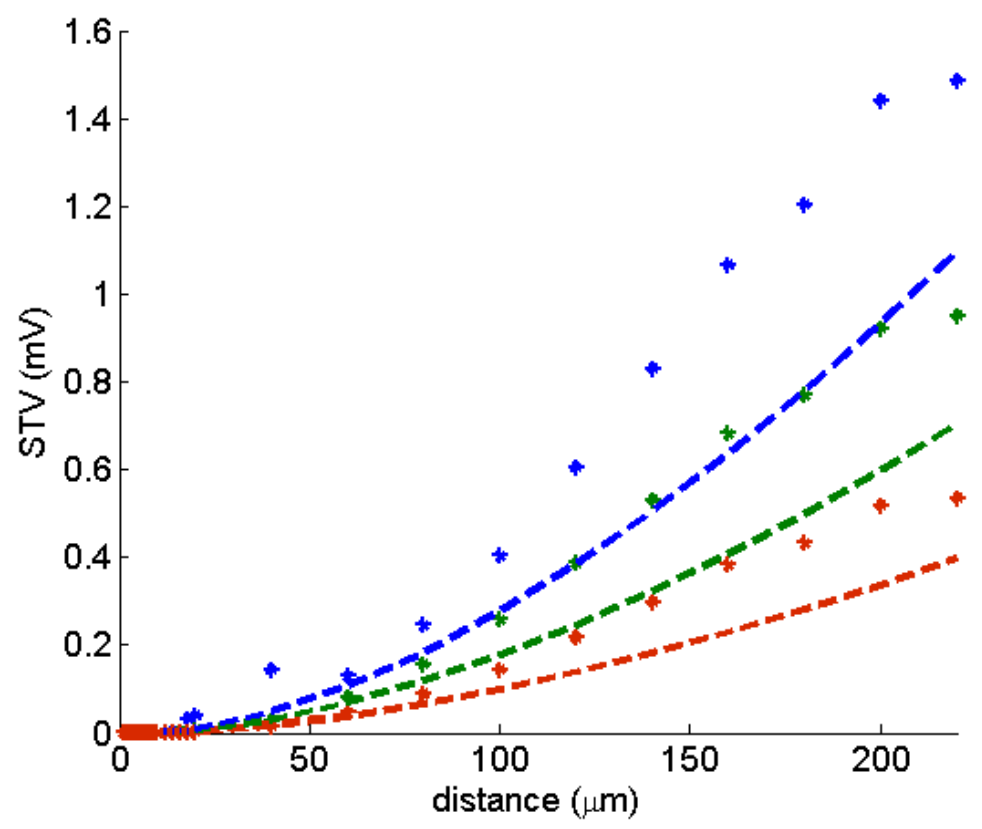

Figure 6.12: Dependence of STV on $\sigma_{I}$ values in passive BST model. $\Psi=$ $0 \mathrm{mV}$. Dashed lines represent the analytical results whereas the dots represent the numerical results. red: $\sigma_{I}=6 \mathrm{pA}$; green: $\sigma_{I}=8 \mathrm{pA}$; blue: $\sigma_{I}=10 \mathrm{pA}$. 


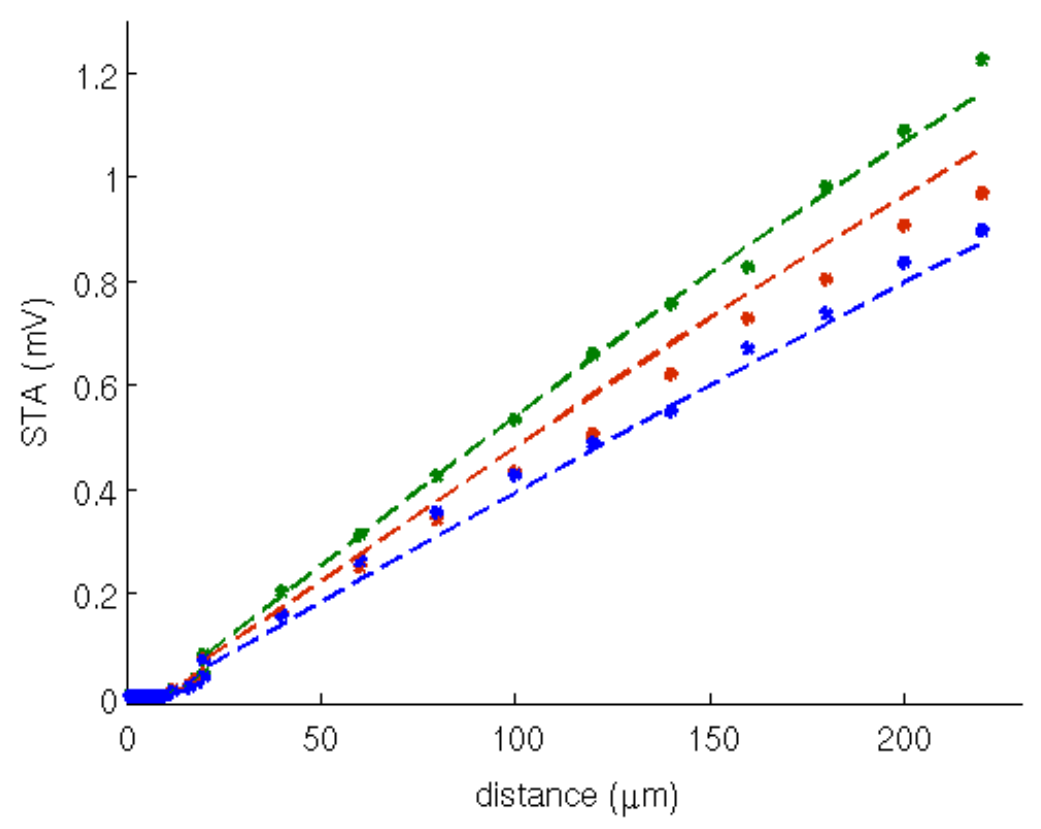

Figure 6.13: Dependence of STA on $\tau_{I}$ values in passive BST model. $\Psi$ $=0 \mathrm{mV}$. Solid lines represent the analytical results whereas the dots represent the numerical results. green: $\tau_{I}=5 \mathrm{~ms}$; red: $\tau_{I}=10 \mathrm{~ms}$; blue: $\tau_{I}=20 \mathrm{~ms}$.

well,which was also the case with STV. Also the dependence on $\tau_{I}$ for both STA and $\mathrm{STV}$ is rather indicated a nonlinear pattern when compared to the dependence on $\sigma_{I}$.

\subsubsection{Impact of Somatic Geometry}

The soma diameter was $10 \mu \mathrm{m}$ in the simulations mentioned in the other sections and the axonal diameter was $1 \mu \mathrm{m}$ in all cases. For measuring the impact of somatic geometry on the spike statistics, the diameter of the soma is altered in a range of $10 \mu \mathrm{m}$ to $1 \mu \mathrm{m}$. It is obvious that when the soma diameter is $1 \mu \mathrm{m}$, the model becomes simply a homogeneous cable.

The change in the somatic surface area did not have an effect on STA values. On the other hand, the increase in the soma diameter led to a decrease in STV values. However, this decrease occured only when the compartment $x$, on which the threshold $\Psi$ was set, was more than $200 \mu \mathrm{m}$ away (Figure 6.15). 


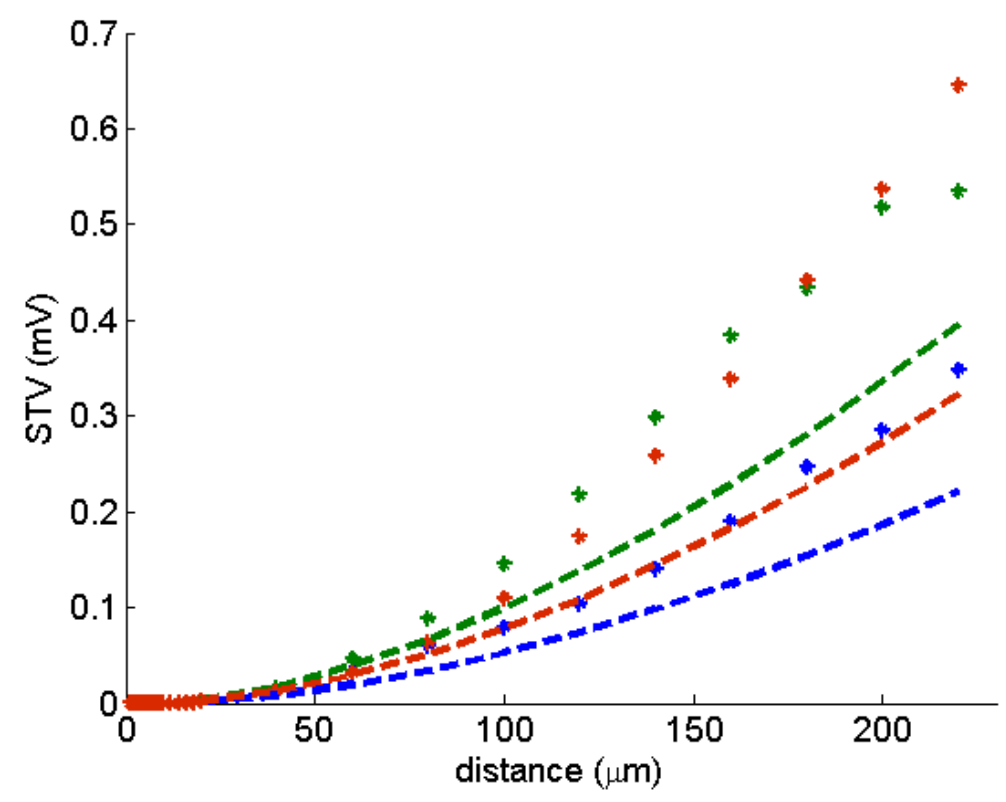

Figure 6.14: Dependence of STV on $\tau_{I}$ values in passive BST model. $\Psi$ $=0 \mathrm{mV}$. Solid lines represent the analytical results whereas the dots represent the numerical results. green: $\tau_{I}=5 \mathrm{~ms}$; red: $\tau_{I}=10 \mathrm{~ms}$; blue: $\tau_{I}=20 \mathrm{~ms}$.

\subsection{Response Properties in the Active Model}

All the active conductances were enabled for the active model. Two modes were alternatingly used for the following simulations :

1. The all-active model : All the compartments had active conductances. However, this model was not useful for the analysis of spike-triggered statistics.

2. The partially-active model : Only one compartment had active conductances and the rest of the model was passive. This model was used to study the passive propagation of action potentials and the action potential generation without active contribution from neighboring compartments.

\subsubsection{All-Active Model}

In Figure 6.16 and 6.17, the propagation of an action potential from somatic end to the axonal end of the model could be seen. The peak value of the action potentials increased by distance from somatic end. The action potentials were initiated at the soma, exactly at the site of current injection. 

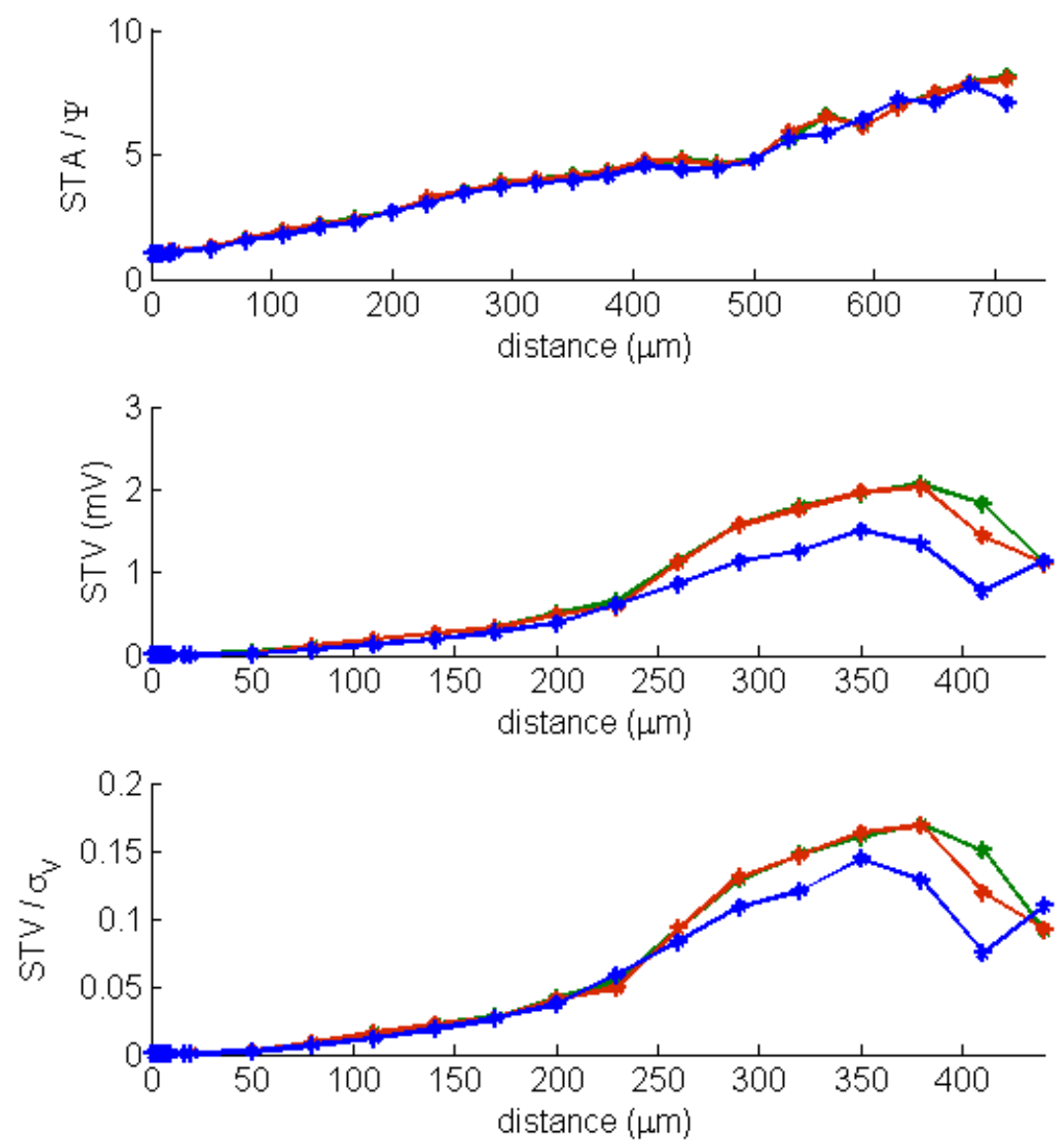

Figure 6.15: Dependence of numerically estimated STA and STV on the somatic geometry. STA and STV values for the model with varying somatic diameters ( $d=1 \mu \mathrm{m}$ (green), $d=1.5 \mu \mathrm{m}$ (red), $d=10 \mu \mathrm{m}$ (blue) ) were estimated for noisy current injection $\left(\sigma_{I}=6 \mathrm{pA}, \tau_{I}=5 \mathrm{~ms}, \mu_{I}=0\right)$ in passive BST model. 


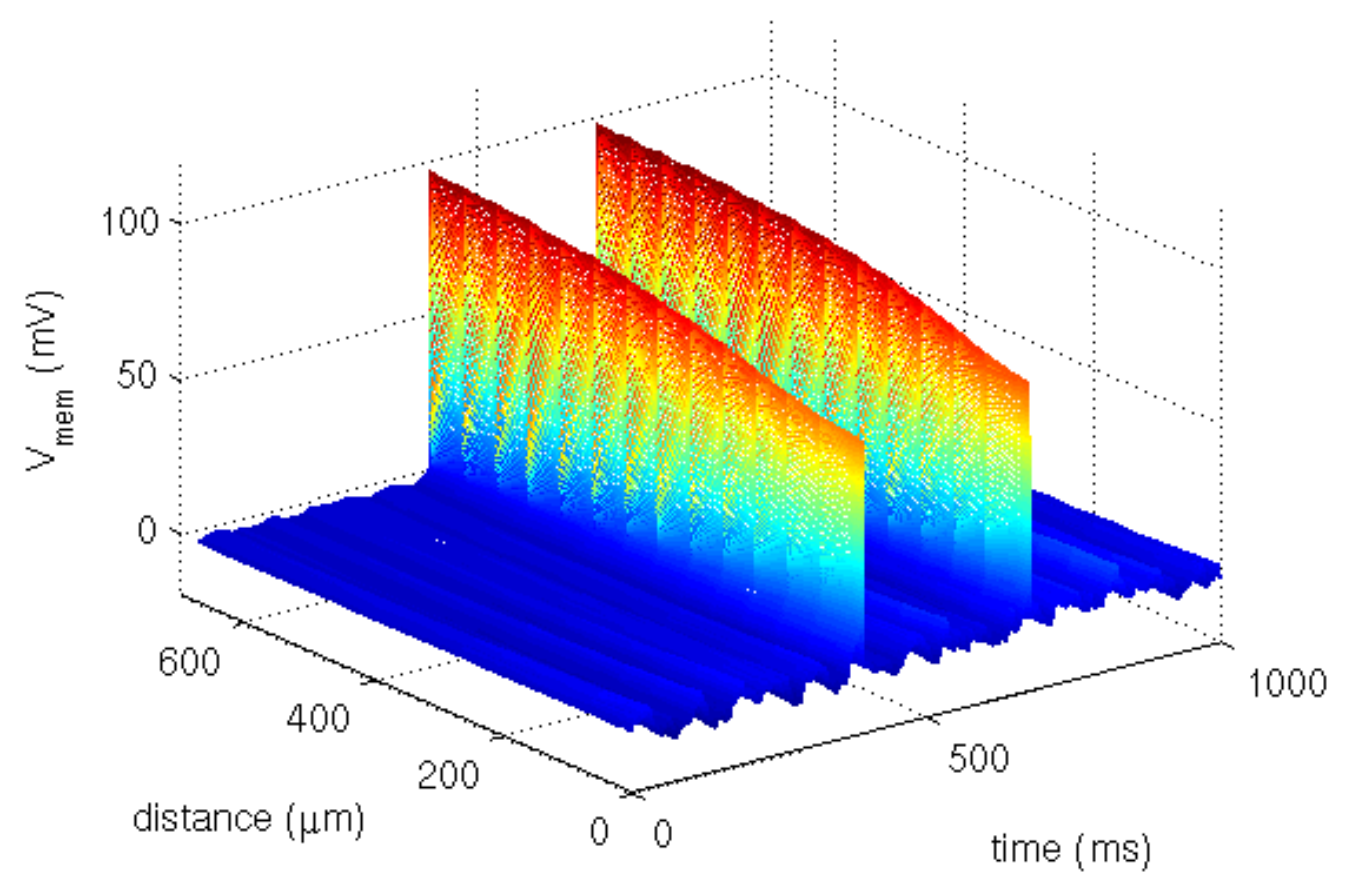

Figure 6.16: 3-D representation of the action potential propagation in allactive BST model. The action potentials were generated under noisy current injection $\left(\sigma_{I}=5.5 \mathrm{pA}, \tau_{I}=20 \mathrm{~ms}, \mu_{I}=1 \mathrm{pA}\right)$. 


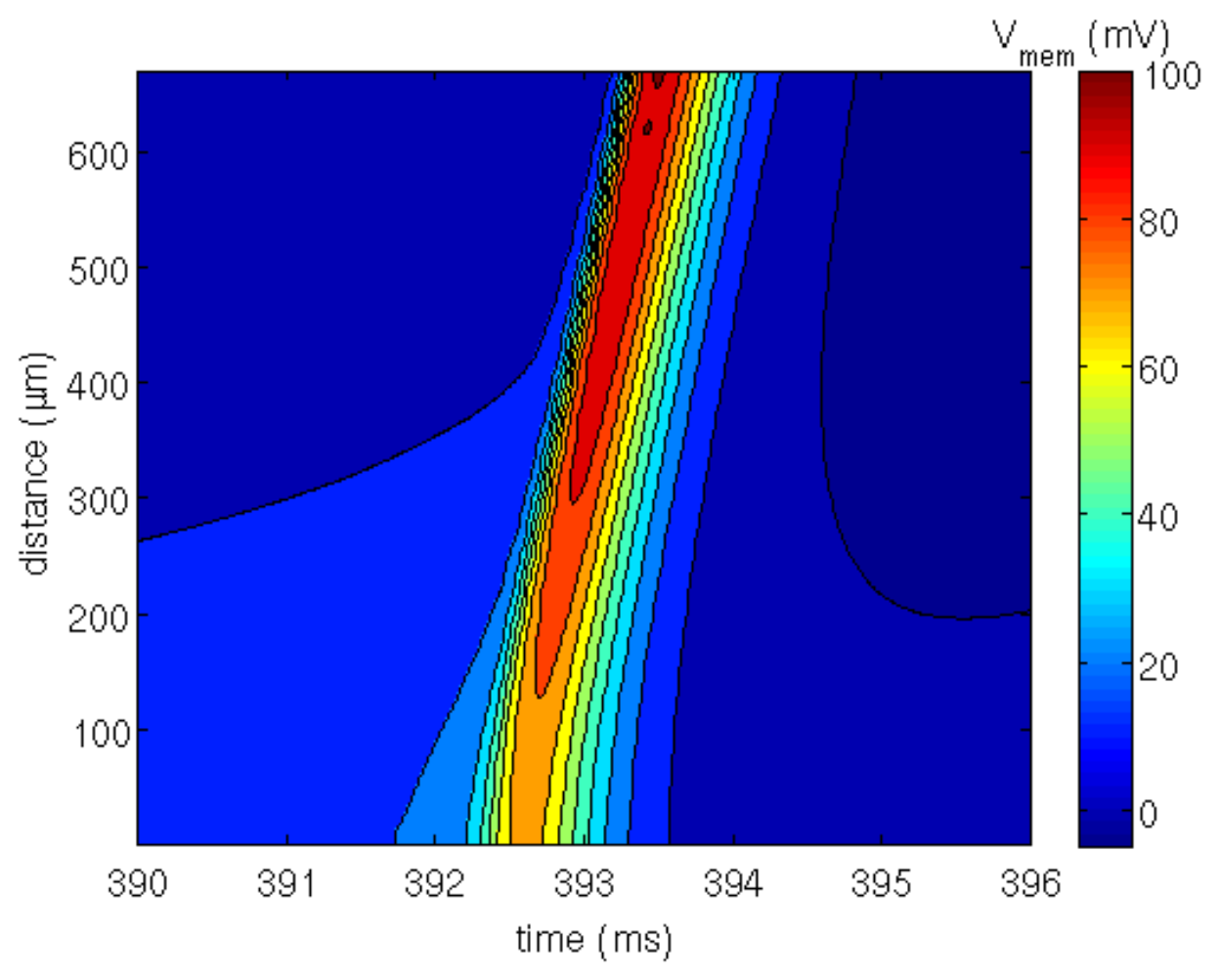

Figure 6.17: Contour plot representation of a single action potential propagation in all-active BST model. The action potential was generated under noisy current injection $\left(\sigma_{I}=5.5 \mathrm{pA}, \tau_{I}=20 \mathrm{~ms}, \mu_{I}=1 \mathrm{pA}\right)$. 


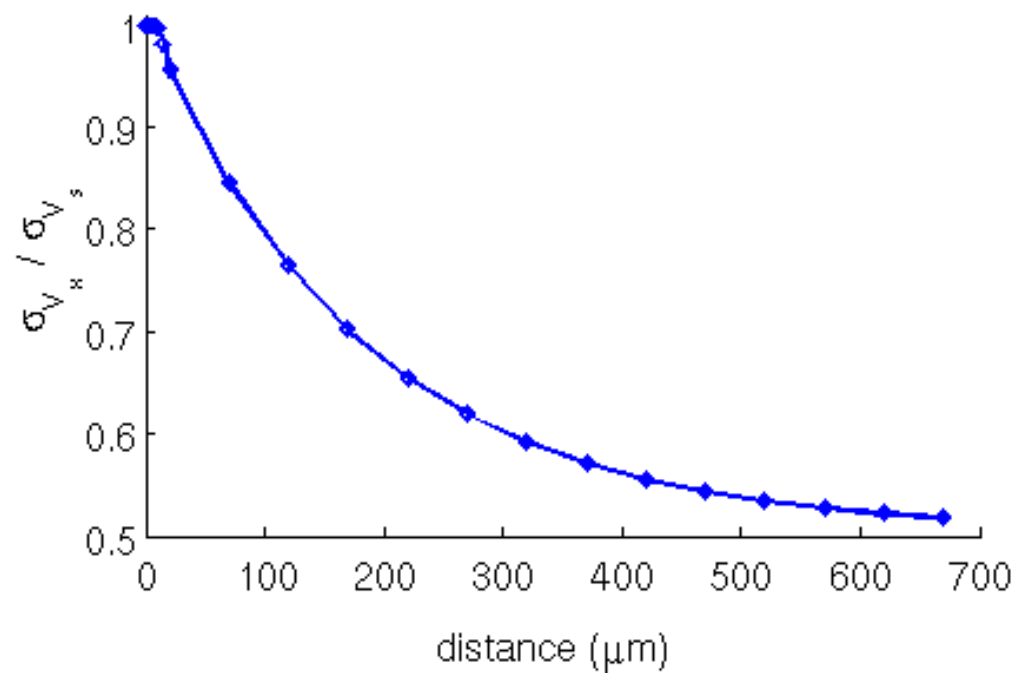

Figure 6.18: Attenuation of $\sigma_{V_{x}}$ by distance from somatic end in all-active BST model. The decay is exponentially dependent on the distance and $\sigma_{V_{x}}$ decreases to approximately $0.5^{*} \sigma_{V_{s}}$ after $670 \mu \mathrm{m}$. The action potentials were generated under noisy current injection $\left(\sigma_{I}=5.5 \mathrm{pA}, \tau_{I}=20 \mathrm{~ms}, \mu_{I}=1 \mathrm{pA}\right)$.

As it could also be concluded from Figure 6.16, the standard deviation for the subthreshold oscillations in a compartment $x$ on the axon, $\sigma_{V_{x}}$, decreased as the distance from somatic end increased. $\sigma_{V_{x}} / \sigma_{V_{s}}$ showed an exponential decay with increasing distance and $\sigma_{V_{x}}$ decreased to approximately the half of $\sigma_{V_{s}}$ after $670 \mu \mathrm{m}$ (Figure 6.18).

For the simulations in all active ball-and-stick model, the channel densities were homogeneous and $\bar{g}_{N a}$ was $300 \mathrm{pS} / \mu \mathrm{m}^{2}$ for all the compartments. Therefore, the action potential were always initiated at the site of current injection and then propagated to the other compartments. On the other hand, the estimation of somatic spike-triggered statistics demands an action potential initiation at the chosen compartment $x$. Hence, the all active ball-and-stick model can not be used for the numerical estimations of STA and STV. This problem was solved by creating a partially active model, such that only the compartment $x$ is active and therefore, the action potentials are initiated at this compartment.

\subsubsection{Partially-Active Model}

In the partially active model, each time a compartment $i$ was chosen in the model to be active and all the other compartments were left passive. This type of model 


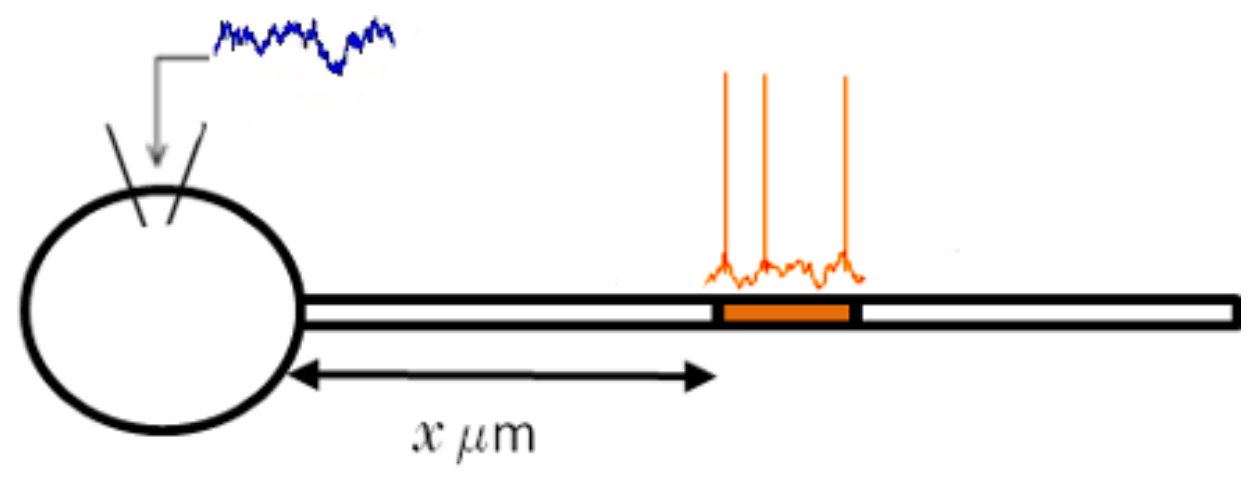

Figure 6.19: The graphical explanation of partially-active BST model. On the ball-and-stick model, a compartment $i$ that is $x \mu \mathrm{m}$ away from soma was chosen to have $\mathrm{Na}^{+}, \mathrm{K}^{+}$and leak conductances (active compartment). All the other compartments remained to have only the leak conductance (passive compartments). When the noisy current was injected into the soma, the action potentials were generated only at the active compartment and then back-propagated to the soma and forwardpropagated to the axon. (The active compartment is represented with orange color in the figure.) Since each compartment that is more than $10 \mu \mathrm{m}$ away from soma had a length of $10 \mu \mathrm{m}$ (Figure 6.2), the active compartment was actually an active region of $10 \mu \mathrm{m}$ length. (Please note that if a compartment in the axon hillock or in the soma was chosen to be active, then the active region was only of $1 \mu \mathrm{m}$ length (Figure 6.2).)

allowed us to study

- Passive propagation of real action potentials;

- Action potential generation without active contribution from neighboring compartments;

- Isolated response properties of a single active compartment.

For all the reasons mentioned above, the partially active ball-and-stick model is appropriate to study the spike-triggered statistics.

\section{Action Potential Propagation in the Partially-Active Model}

The action potentials were generated in the active compartment $i$, that is at $x_{i} \mu \mathrm{m}$ distance from the somatic end of the model (Figure 6.19). The attenuation of the action potentials were visible at the soma for $x_{i} \geq 40 \mu \mathrm{m}$ (Figure 6.21). For $x_{i} \geq$ $380 \mu \mathrm{m}$, there were no more action potentials in the soma. This value is close to the 


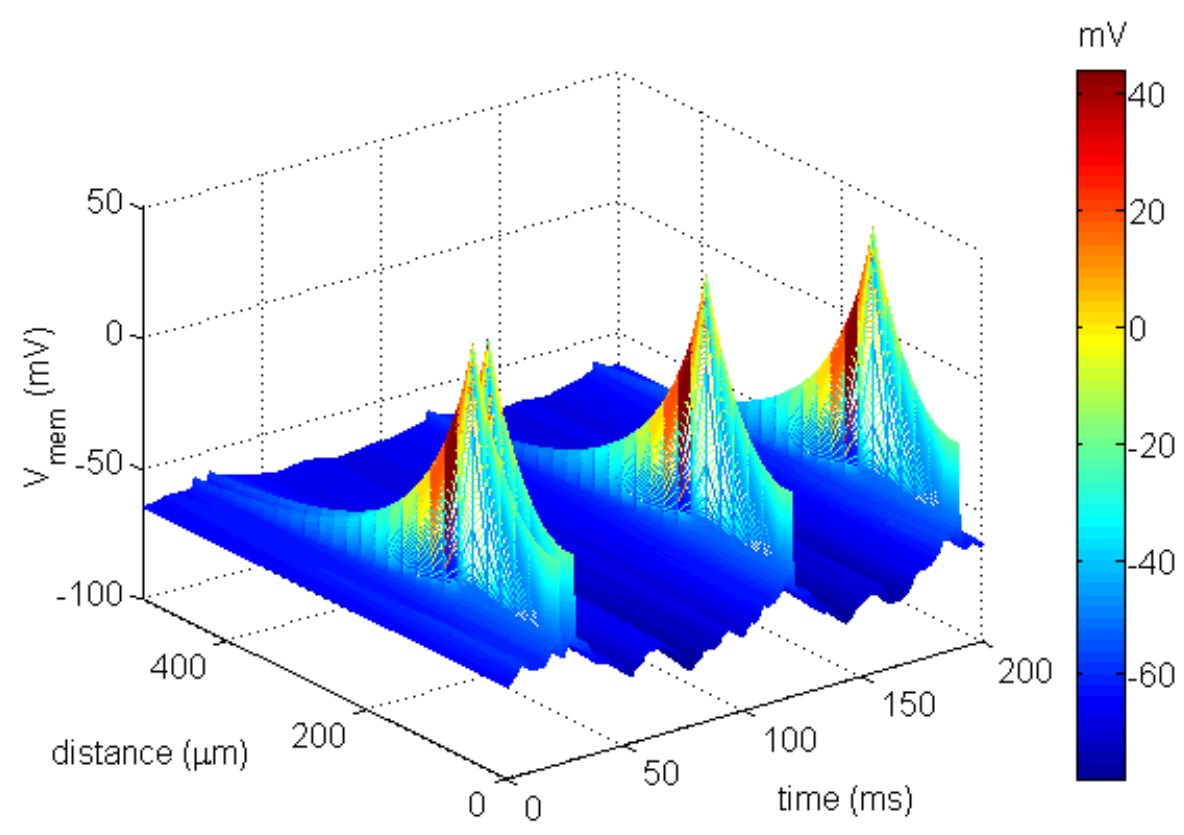

Figure 6.20: 3-D representation of the action potential propagation in partially-active BST model. The action potentials were generated under noisy current injection $\left(\sigma_{I}=11 \mathrm{pA}, \tau_{I}=5 \mathrm{~ms}, \mu_{I}=0 \mathrm{pA}\right)$. 


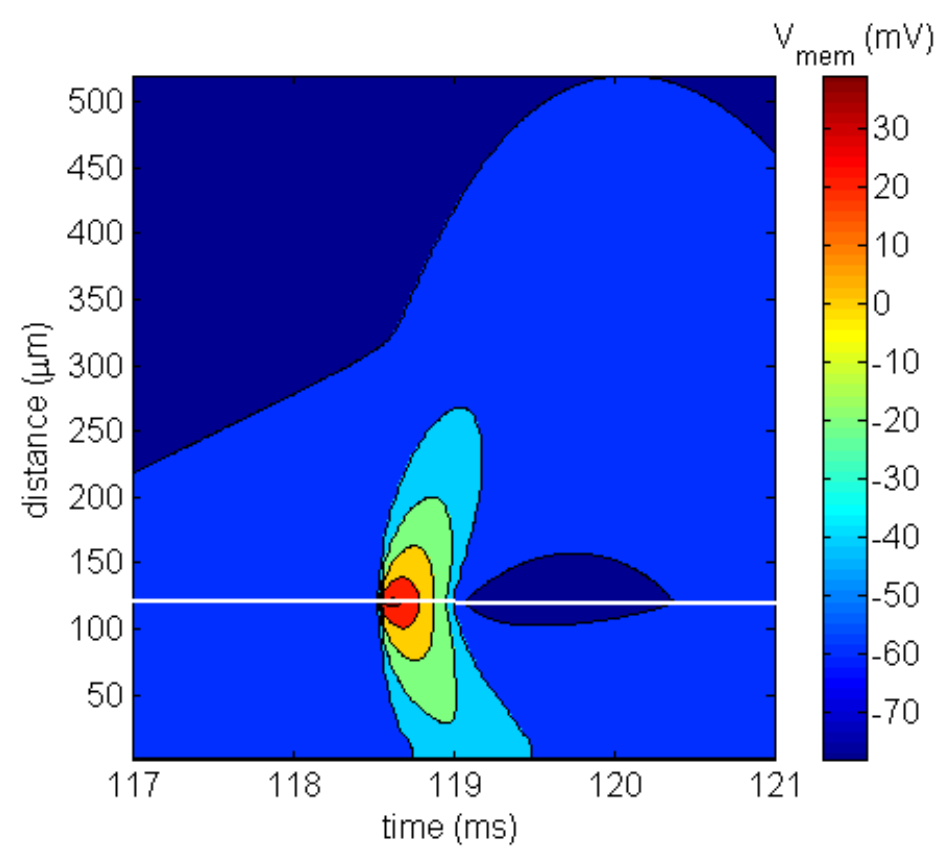

Figure 6.21: Contour plot representation of the action potential propagation from the active compartment in partially-active BST model. The white line indicates the site of active conductance, and therefore, of the action potential initiation. The action potential was generated under noisy current injection $\left(\sigma_{I}=11\right.$ $\left.\mathrm{pA}, \tau_{I}=5 \mathrm{~ms}, \mu_{I}=0 \mathrm{pA}\right)$. 
electrotonic length constant $(\lambda \cong 412 \mu \mathrm{m})$, therefore it might be concluded that the real action potential can not propagate on a passive model more than the electrotonic length constant, which is as expected.

The action potentials are initiated at $x_{i}=120 \mu \mathrm{m}$. The antidromic conduction velocity was $2.5 \mathrm{~m} / \mathrm{s}$ (Figure 6.23) and $500 \mathrm{~V} / \mathrm{m}$ (Figure 6.22). The axonal electrotonic length constant was measured as $400 \mu$ from Figure 6.22. This value is very close to $\lambda$ $=412 \mu \mathrm{m}$ of the passive BST model. The orthodromic conduction velocity for $x<\lambda$ was $3.45 \mathrm{~m} / \mathrm{s}$ whereas it was $2.04 \mathrm{~m} / \mathrm{S}$ for $x \geq \lambda$. This high conduction velocities are not comparable to the real (e.g. cortical) neurons, which have a conduction velocity in the range of $1 \mathrm{~m} / \mathrm{s}$. However, as action potentials in the partially-active BST model propagate on a fully passive membrane and as the model has a very simple geometry, a higher conduction velocity was anticipated. The two-piece orthodromic conduction velocity stems from the passive conduction on the cable-like axon. It was known that action potentials can not propagate further away from $\lambda$ on a passive cable by definition, which was also supported by our results. Therefore, a lower velocity for the conduction of membrane potential fluctuations (caused by the real action potential in active compartment) towards the axonal compartments at $x>\lambda$ was also anticipated.

\section{Spike-Triggered Statistics in Partially-Active Model}

As also in the all-active model, $\sigma_{V_{x}} / \sigma_{V_{s}}$ decreases by increasing distance from the initiation site, which is located at $x_{i}=120 \mu \mathrm{m}$. Due to the different geometry between soma and axon, this decrease on antidromic and orthodromic directions can not be symmetrical (Figure 6.25) and

$$
\begin{aligned}
\sigma_{V_{x_{i}}} & =1.35 * \sigma_{V_{s}} \\
\sigma_{V_{x_{i+\lambda}}} & =0.25 * \sigma_{V_{s}}
\end{aligned}
$$

The numerical STA and STV estimations for the partially-active BST model are given in Figure 6.26. As in the passive BST model, STA is dependent on threshold. STV values are identical for all threshold values until $75 \mu \mathrm{m}$. Even after this point, the difference between STVs for different threshold values is negligible. These results also agree with the passive BST model.

\subsection{Dynamic Response in Partially-Active BST Model}

The response to the sinusoidally modulated input current (see section 6.1.7) was obtained from the partially-active BST model at $x=4 \mu \mathrm{m}$ and $x=40 \mu \mathrm{m}$ away from 


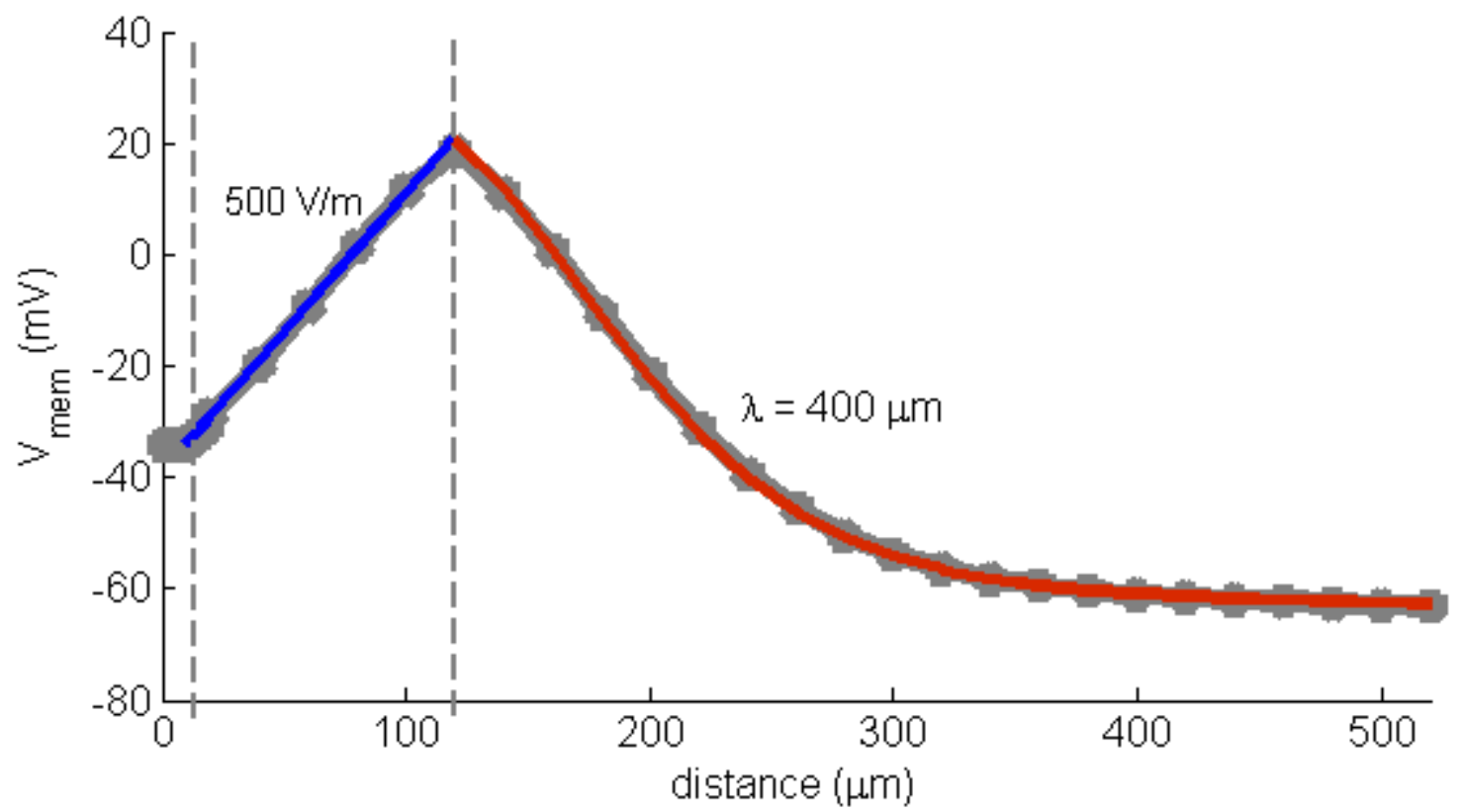

Figure 6.22: The conduction of maximum potential in partially-active BST model. The active compartment was located at $x_{i}=120 \mu \mathrm{m}$. The dashed gray lines indicate the distance from soma-axon meeting point to $x_{i}$. The fit lines were obtained by linear regression of $y_{1}=v_{a d} * x+b_{1}$ (blue) and $y_{2}=a_{2} * \exp (x / \lambda)-b_{2}($ red $)$. $v_{a d}$ was $500 \mathrm{~V} / \mathrm{m}$ and $\lambda$ was $400 \mu \mathrm{m}$. The action potential was generated by noisy current injection into the soma $\left(\sigma_{I}=11 \mathrm{pA}, \tau_{I}=5 \mathrm{~ms}, \mu_{I}=0 \mathrm{pA}\right)$. 


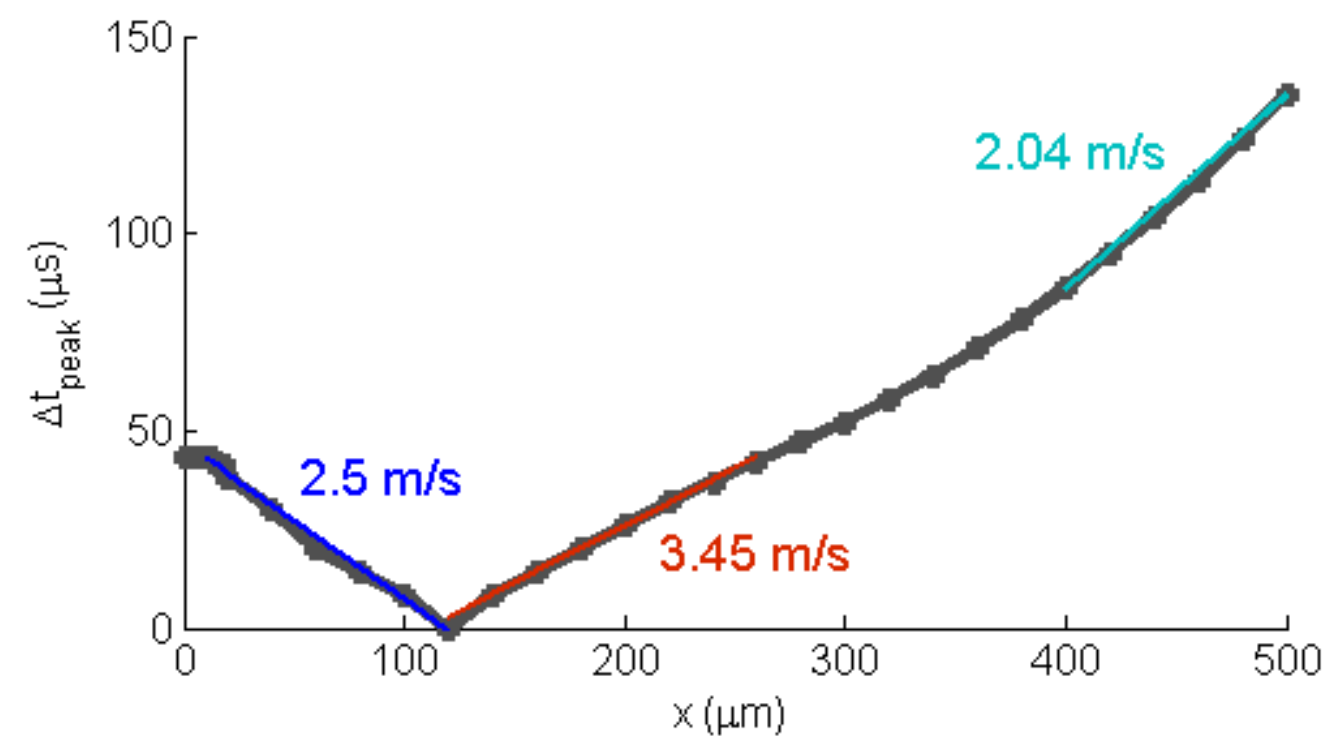

Figure 6.23: Onset latency in partially-active BST model. The active compartment was located at $x_{i}=120 \mu \mathrm{m}$. The action potential then propagated from $x_{i}$ in antidromic and orthodromic directions. The fit lines were obtained by the linear regression of $y_{a d}=v_{a d} * x+b_{1}$ (blue), $y_{o d, 1}=v_{o d, 1} * x+b_{2}($ red $)$ and $y_{o d, 2}=v_{o d, 2} * x+b_{3}$ (light blue). $v_{a d}$ was $2.5 \mathrm{~m} / \mathrm{s}$ whereas $v_{o d, 1}$ was $3.45 \mathrm{~m} / \mathrm{s}$ and $v_{o d, 2}$ was $2.04 \mathrm{~m} / \mathrm{s}$. The action potential was generated by noisy current injection into the soma $\left(\sigma_{I}=11 \mathrm{pA}\right.$, $\left.\tau_{I}=5 \mathrm{~ms}, \mu_{I}=0 \mathrm{pA}\right)$.

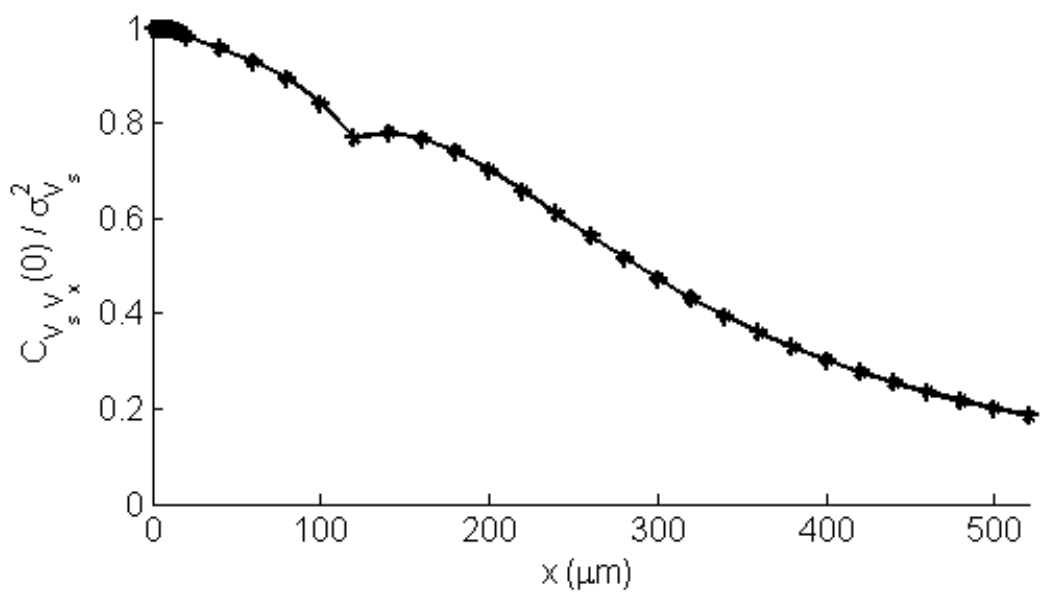

Figure 6.24: The normalized soma-axon covariance in partially-active BST model. The covariance $C_{V_{s} V_{x}}(0)$ was normalized as $C_{V_{s} V_{x}}(0) / \sigma_{V}^{2}$. The statistics were obtained under noisy current injection into the soma $\left(\sigma_{I}=11 \mathrm{pA}, \tau_{I}=5 \mathrm{~ms}, \mu_{I}=\right.$ $0)$. 


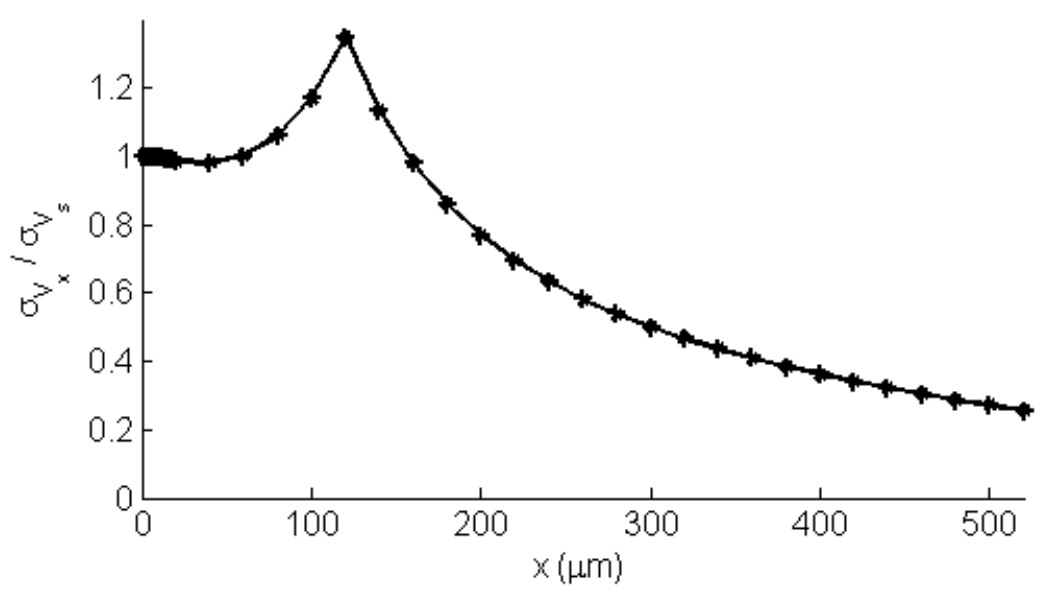

Figure 6.25: Attenuation of $\sigma_{V_{x}}$ by distance from active compartment in partially-active BST model. The active compartment was located at $x_{i}=120$ $\mu \mathrm{m}$. The decay was exponentially dependent on the distance and was not symmetrical in antidromic or orthodromic directions. $\sigma_{V_{x_{i}}}$ was $1.35^{*} \sigma_{V_{s}}$ and $\sigma_{V_{x_{i+\lambda}}}$ was $0.25^{*} \sigma_{V_{s}}$. The action potential was generated by noisy current injection into the soma $\left(\sigma_{I}=11\right.$ $\left.\mathrm{pA}, \tau_{I}=5 \mathrm{~ms}, \mu_{I}=0 \mathrm{pA}\right)$.

soma. Data were collected in separate trials where each time only one compartment was active.

It was expected that BST model exhibits a low-pass filter characteristic as in the real (e.g. cortical) neuron. The results supported the predictions (Figure 6.28).

The steady state firing rate, $\nu_{0}$, was $10 \mathrm{~Hz}$ in both cases. This also corresponds to the cut-off frequency, $f_{\text {cut }}$, for both compartments (Figure 6.28). Although passive electrical properties, geometry and channel denstities are same for both compartments, because of their distance from soma, $x=4 \mu \mathrm{m}$ was considered as the axon hillock and $x=40 \mu \mathrm{m}$ was considered as the axon initial segment. With this consideration, it was expected that axon hillock should have a slightly slower decay in the gain compared to axon initial segment. The resulting curves supported this prediction.

To sum up, although the gain for axon hillock was slightly higher for axon hillock than axon initial segment after $f_{c u t}$, the difference in $\nu_{1} / \nu_{0}-f_{I}$ curves of two compartments that are proximal to the soma and $36 \mu \mathrm{m}$ away from each other was very small. 

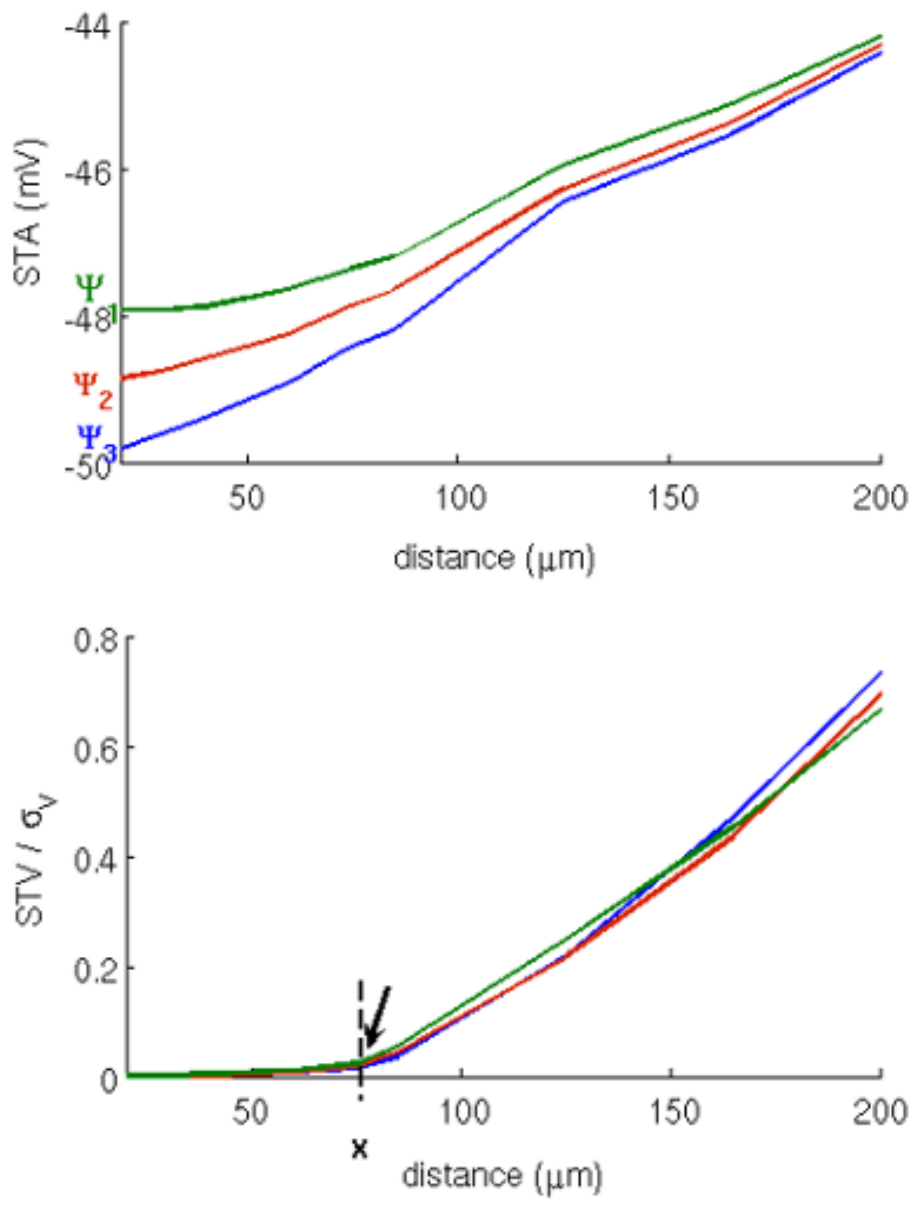

Figure 6.26: Dependence of STA (upper figure) and STV (lower figure) on threshold variability and distance from somatic end in partially-active BST model. Upper figure: Numerical results of STA for threshold values $\Psi_{1} \cong-48 \mathrm{mV}$ (green), $\Psi_{2} \cong-49 \mathrm{mV}$ (red) and $\Psi_{3} \cong-50 \mathrm{mV}$ (blue). Lower figure: Numerical results of STV is normalized with $\sigma_{V}$ and shown for the threshold values given above. The arrow and the dashed line indicates the point where the results for different threshold values are no longer identical $(x=75 \mu \mathrm{m})$. The traces were obtained by noisy current injection $\left(\sigma_{I}=5 \mathrm{pA}, \tau_{I}=20 \mathrm{~ms}, \mu_{I}=0\right)$. 

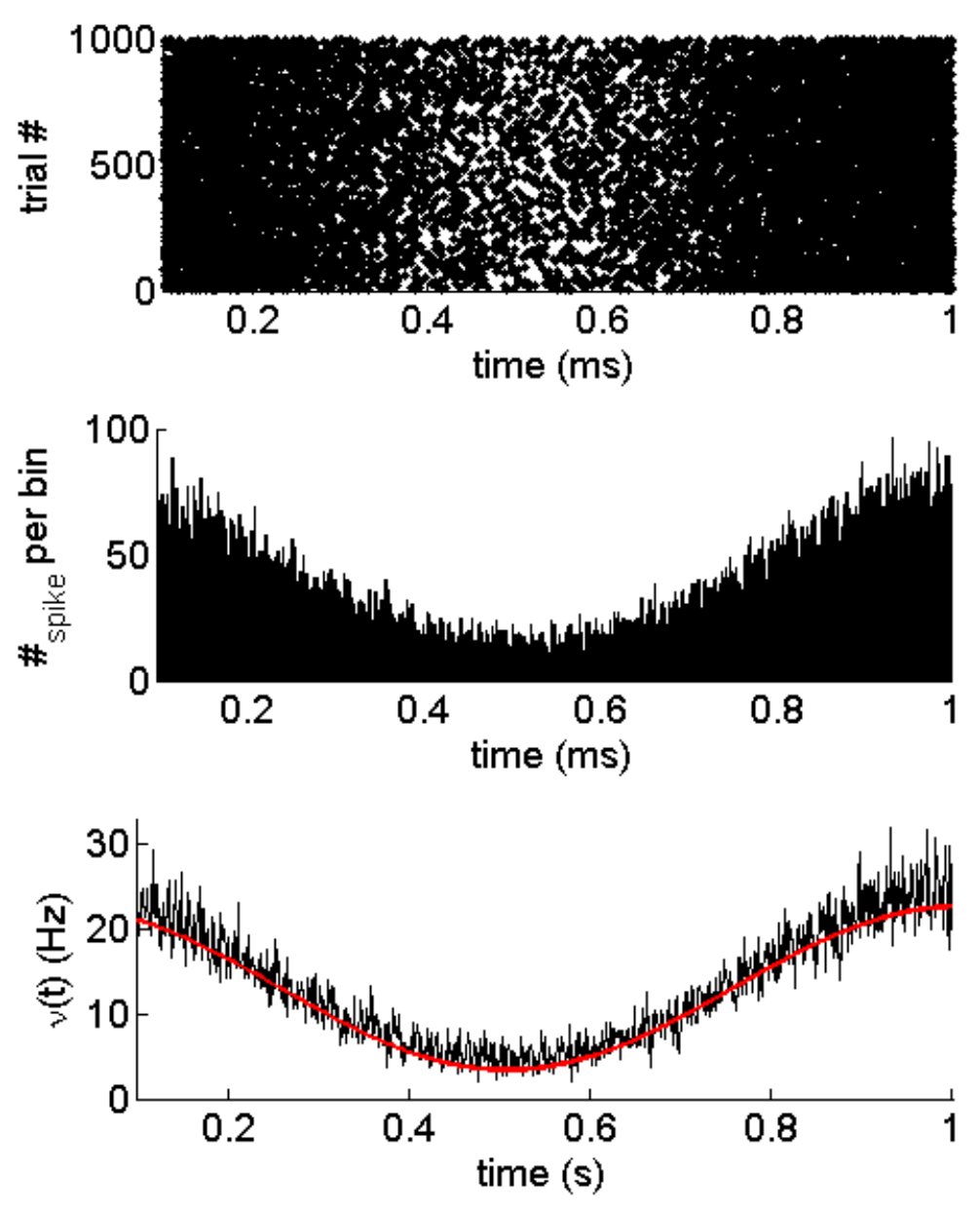

Figure 6.27: Typical examples of Raster Plot (uppermost), PSTH (middle) and $\nu(\mathbf{t})$-t plot(lowermost). The graphs are obtained from axon initial segment $(\mathrm{x}=40 \mu \mathrm{m})$ by injecting a sinusoidally fluctuating noisy current $\left(\mathrm{f}_{I}=1 \mathrm{~Hz}, \mathrm{I}_{I}=10\right.$ $\left.\mathrm{pA}, \sigma_{I}=11 \mathrm{pA}, \tau_{I}=5 \mathrm{~ms}, \mu_{I}=0\right)$ to the soma. The steady state firing rate was $10 \mathrm{~Hz}$. The method is explained in more detail in section 6.1.7 


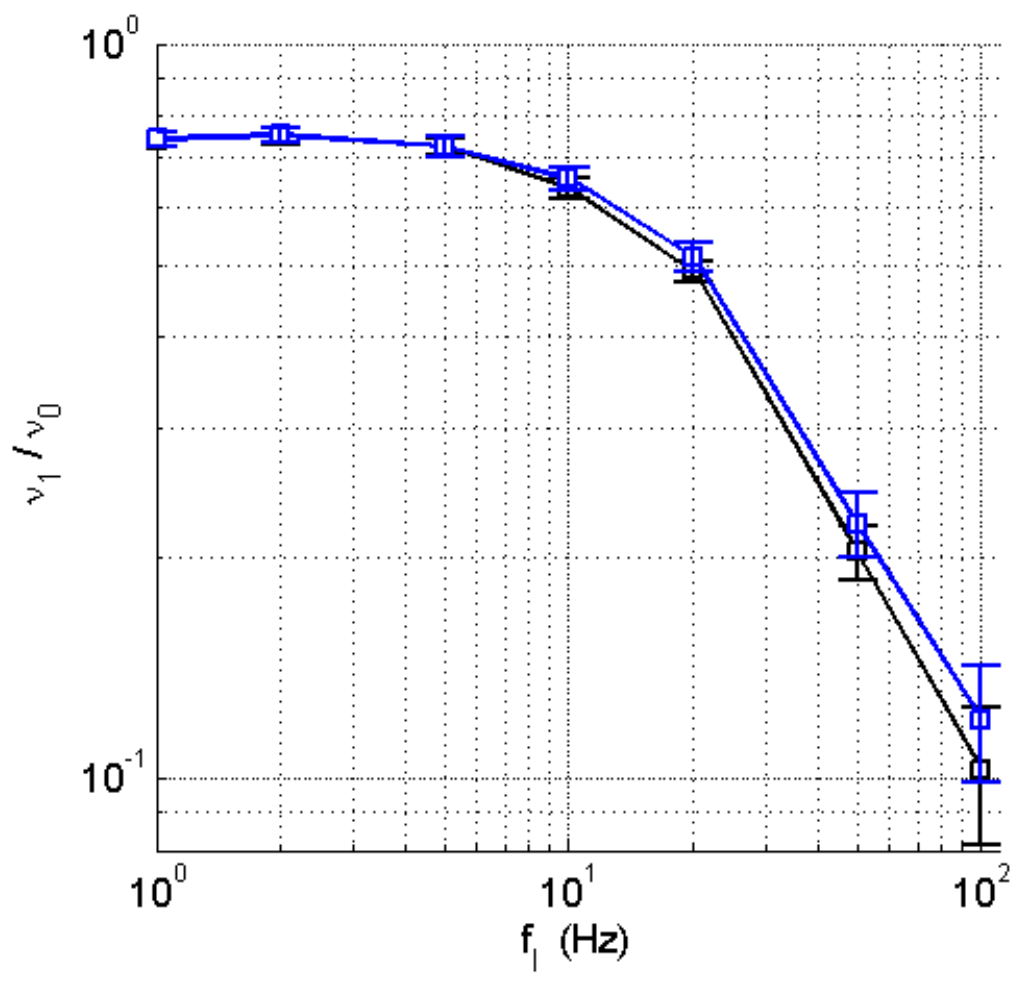

Figure 6.28: Gain modulation under sinusoidally modulated input. Blue: axon hillock, black: axon initial segment. $\sigma_{I}=11 \mathrm{pA}, \tau_{I}=5 \mathrm{~ms}, \mu_{I}=0 \mathrm{pA}, I_{1}=$ $10 \mathrm{pA}, \nu_{0}=10 \mathrm{~Hz}$ 


\subsection{Summary and Discussion}

The most important implication of the results presented in this chapter is that the somatic and axonal potentials at the action potential onset does not differ significantly if the distance from soma $x$ is less than $100 \mu \mathrm{m}$.

The results presented in this chapter indicates some important aspects of the implications of spike-triggered statistics in multi-compartmental neuron models:

1. Spike-triggered average (STA) is linearly dependent on the choice of threshold. This conclusion was expected from the definition of STA, and it can be used to validate the implementation of the model.

2. Spike-triggered variance (STV) is independent of the choice of threshold. The numerical and analytical results matched until $200 \mu \mathrm{m}$ from soma and the measured STV was always lower than the analytically estimated STV. This implies that the potential in the soma and in the axon until $200 \mu \mathrm{m}$ away from soma does not differ significantly at the time of threshold crossing.

A recent paper reported intriguing findings about the adjustment of neuronal excitability by relocation of AIS either more proximal or more distal to soma (Grubb and Burrone [71]). In this study, it was claimed that even very small distances such as $5 \mu \mathrm{m}$ could have a significant affect on the neuronal response and the firing rate. However, the results of the simulations discussed in this chapter contradict with this finding. The dynamic response properties of BST model suggest that even $35 \mu \mathrm{m}$ distance is not far enough to generate a significant difference in the dynamic response, e.g. in the cut-off frequency (Figure 6.28). 


\section{Part III}

\section{Numerical Analysis of Cooperative $\mathrm{Na}^{+}$Channel Gating in the Axon Initial Segment}





\section{Abstract}

Ion channels are the building blocks of of the information processing in neurons. Therefore, any realistic neuron model should include effective and reliable ion channel components.Most biophysical models are typically designed to study one particular aspect of channel gating in detail, on the other hand, the neuron modelers require broad coverage of the entire range of channel behavior. To achieve that, the approximate representations of the channel structure-function relationship that omit the structural features of the channels, which can not be properly constrained, are more commonly used. The most popular representation of this type, the Hodgkin-Huxley model, assumes the statistically independent gating of ionic channels. However, recent studies pointed out that synchronous activity of $\mathrm{Na}^{+}$are also observed for various cell types. This synchronous activity means that the activity (e.g. opening) of one channel triggers the activity (e.g. opening) of a certain fraction of its neighbors and hence, these group of channels behave as a unit. Although there is no direct biological proof that this type of cooperative $\mathrm{Na}^{+}$channel activity is also existent in the axon initial segment of cortical neurons, our estimations indicate that such mechanism could be responsible for the high onset rapidness and the action potential waveforms observed in cortical neurons, which can not be explained by the canonical HodgkinHuxley type models. In this study, we explored the cooperative gating hypothesis on two related models : a single-compartment conductance-based model with cooperative $\mathrm{Na}^{+}$channel gating kinetics (SC-COOP, chapter 8) and a multi-compartmental conductance-based model with cooperative gating kinetics at the axon initial segment (MCCAIS, chapter 9). 


\section{7 Cooperative gating of voltage-gated ionic channels}

The cell membrane contains many subpopulations of ionic channels that are heterogeneously distributed.Although the distance between the channels of a certain type is most of the time far enough to exclude a possibility of interaction, it was also shown that some of the ionic channels tend to form clusters. For different cell types from Archea to mammalian neurons, inter-channel coupling was shown to exist in these clusters (Molina et al. [127]; Dekker and Yellen [48]; Saito et al. [155]; Marx et al. [120]; Undrovinas et al. [179]; Huang [87])

The concept of inter-channel coupling contradicts with the basic assumption in the classical Hodgkin-Huxley type models that the channels act statistically independently. However, recent findings indicated that the predictions of classical HodgkinHuxley type formulations for the AP onset and threshold variability do not satisfy the findings for in vivo and in vitro (Naundorf et al. [132]) cortical neurons. Although it is yet to be confirmed by the single channel experiments, recent macroscopic findings suggested that cooperative gating of $\mathrm{Na}^{+}$channels in cortical neurons is a plausible explanation for the generation of such sharp AP onsets and threshold variability in cortical neurons (Naundorf et al. [132]).

The collective behavior of individual neurons in a neural circuit, e.g. synchronization, strongly depends on the AP generating dynamics of a neuron (Ermentrout et al. [56]). Therefore, how this cooperative gating mechanism contribute to the individual neural response and to the large-scale dynamics is yet another intriguing question.

To test the cooperative gating hypothesis using numerical and theoretical tools, a single-compartment and a multi-compartment neuron model was constructed such that Hodgkin-Huxley type conductance algorithms with the implementation of cooperative $\mathrm{Na}^{+}$channel gating kinetics form the skeleton of the models. To further understand the impact of cooperative gating on large-scale dynamics, I analyzed the linear response properties of each model in comparison to the non-cooperative models. 
The single-compartment cooperative (SC-COOP) model maintained a preliminary step for my simulations. I investigated the characteristics of cooperatively gating $\mathrm{Na}^{+}$channels and its effect on the action potential generating mechanisms and on the stationary and dynamic response properties in this simple theoretical framework. The more realistic multi-compartmental cooperative axon initial segment (MCCAIS) model provided with grounds to investigate some important aspects of real neurons, such as :

- The action potential initiation dynamics and the site of initiation,

- The $\mathrm{Na}^{+}$channel density distribution in different parts and the effect of variations in the channel densities,

- The action potential propagation and the conduction velocity,

- The effect of cooperative gating on the characteristics given above.

In this chapter, I will give a brief background on the experimental findings about inter-channel coupling. Then, I will emphasize the possible outcomes of cooperative gating hypothesis, that are relevant to the neural transfer functions and to the efficient neural encoding.

\subsection{Experimental Evidence}

\subsection{1 $\mathrm{K}^{+}$channels}

Simple homologue of mammalian ion channels, that can be found on the extremophyle bacteria and that are significantly resistant to tough experimental conditions provided an insight about the channel structures ad function. For instance, a $\mathrm{K}^{+}$ channel from the soil bacteria Streptomyces lividans, KcsA, was shown to exhibit unexpectedly complicated opening and closing patterns, although it has the simplest $\mathrm{K}^{+}$channel structure known up to date (Doyle et al. [50] ; Molina et al. [127]). The channel exhibits two distinct patterns : Low-opening-probability (LOP) and highopening-probability (HOP), where the low or high probability was defined upon the probability of finding channel opening events in the recordings. A single channel current was measured as $4 \mathrm{pA}$. In LOP pattern, both 4 and 8 pA closing and opening events with single channel-like appearance were observed. The peak amplitude histogram would display a binomial distribution if all the events were due to independent opening of single channels. However, the histogram gave two peaks at 4 and $8 \mathrm{pA}$, suggesting the positive coupling of two channels. In HOP pattern, a positive coupling of up to 5 channels was observed and was statistically confirmed using the 


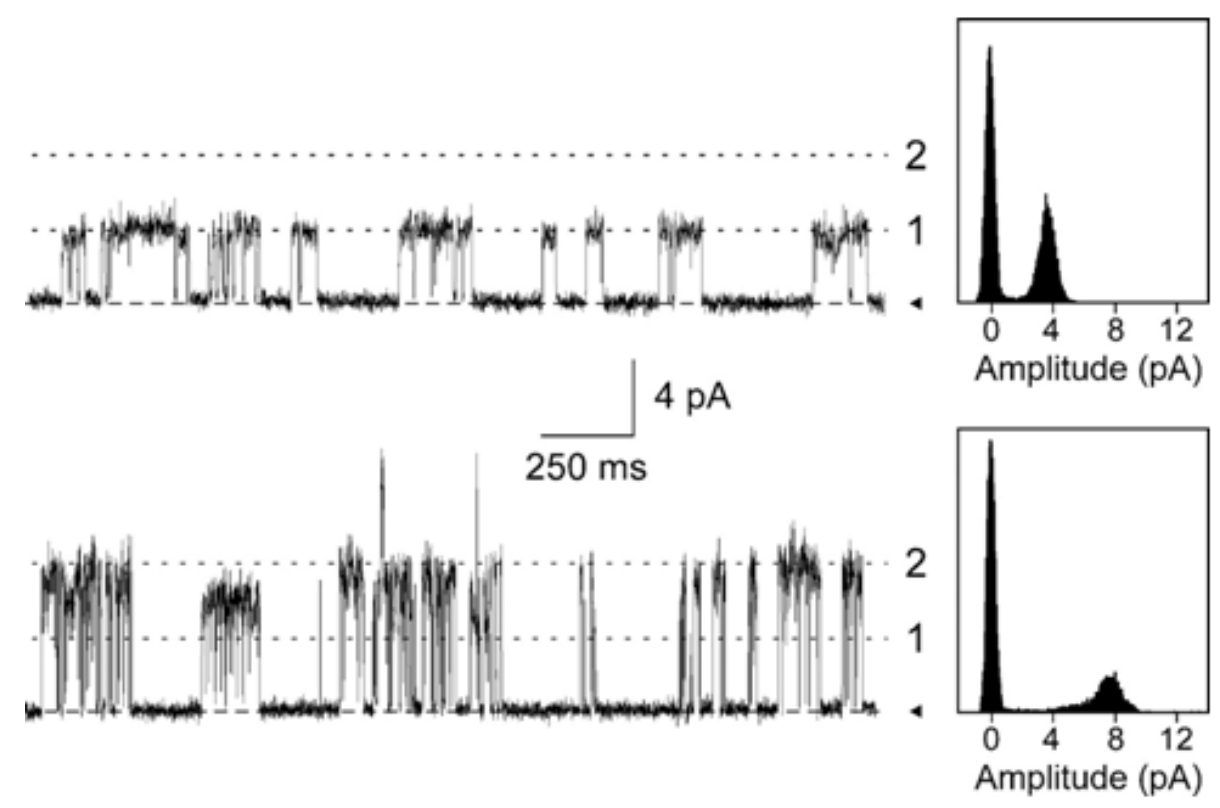

Figure 7.1: Coupled gating of KcsA channels in the LOP pattern. Upper figure: a recording containing only the minimal 4-pA single channel currents seen at +150 mV; Lower figure: a recording obtained under identical conditions but containing predominant 8-pA currents resulting from coupling of two KcsA channels. The peak amplitude histograms of each of the above recordings are shown to the right. The latter 8-pA currents were the predominant events in most recordings of KcsA taken in LOP patterns. The dashed lines in the two recordings (labeled ' 1 ' and '2') indicate the 4- and 8-pA current levels. Adapted from Molina et al. [127].

same method. This study was performed on excised membrane patches from reconstituted giant liposomes containing purified KcsA. To show the clustering on the lipid membrane, the authors performed also confocal microscopy and FRET measurements (Molina et al. [127]). The results indicated clusters of KcsA channels on homogeneous lipid membrane.

Inter-channel coupling was also shown for yet another $\mathrm{K}^{+}$channel : hyperpolarizationactivated cyclic nucleotide-gated (HCN) channel (Dekker and Yellen [48]). The study on HEK 293 cells showed that the single channel recordings in respond to a voltage step resulted in variable delays with no channel openings which appeared highly correlated in time. This coupled activity was also evident when the stochastic fluctuations in microscopic currents were analyzed. The authors suggested some explanations for the correlated channel openings. They claimed that the possibility of intra-channel (or inter-subunit) coupling rather than inter-channel (or inter-protein) coupling was unlikely due to the independent observations of single channel openings as well as 

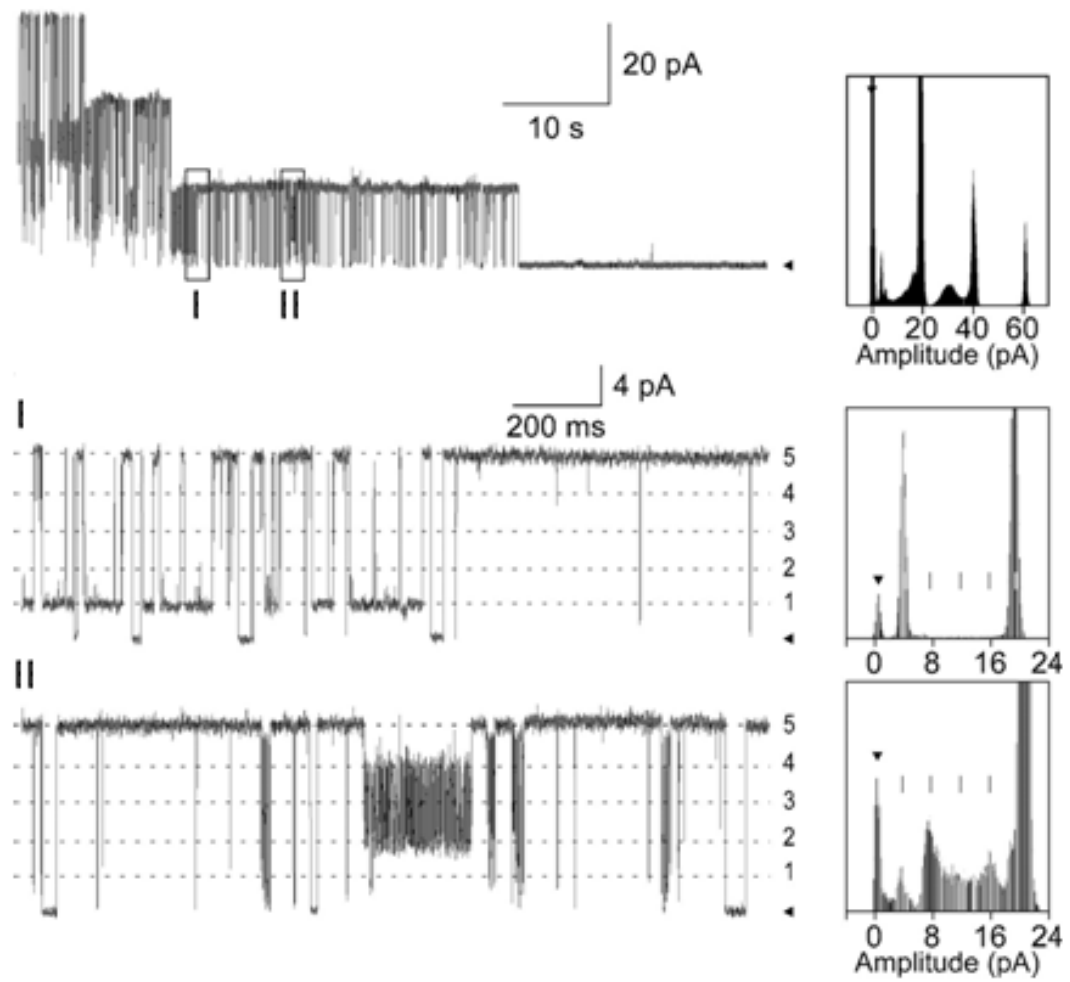

Figure 7.2: Coupled gating of KcsA channels in HOP patterns. Uppermost figure: a representative recording taken at $+150 \mathrm{mV}$ illustrating three successive closings of 20-pA current levels in a HOP pattern similar to that shown in Figure 7.1. I and II, some regions of the above recording in more detail, in which, in addition to the main 20-pA current level, openings and closings of a smaller 4-pA current could also be observed, along with other currents whose intensities are integer multiples of the smaller 4-pA currents. The main gating event, however, corresponds to the 20-pA currents, which seemingly result from the positive coupling of five of the smaller 4-pA current levels. The amplitude histograms of each of the above recordings are shown to the right. Dashed lines in B and C (flanked by numbers ' 1 ' through ' 5 ') are solely to indicate the 4-, 8-, 12-, 16-, and 20-pA current levels.Adapted from Molina et al. [127]. 

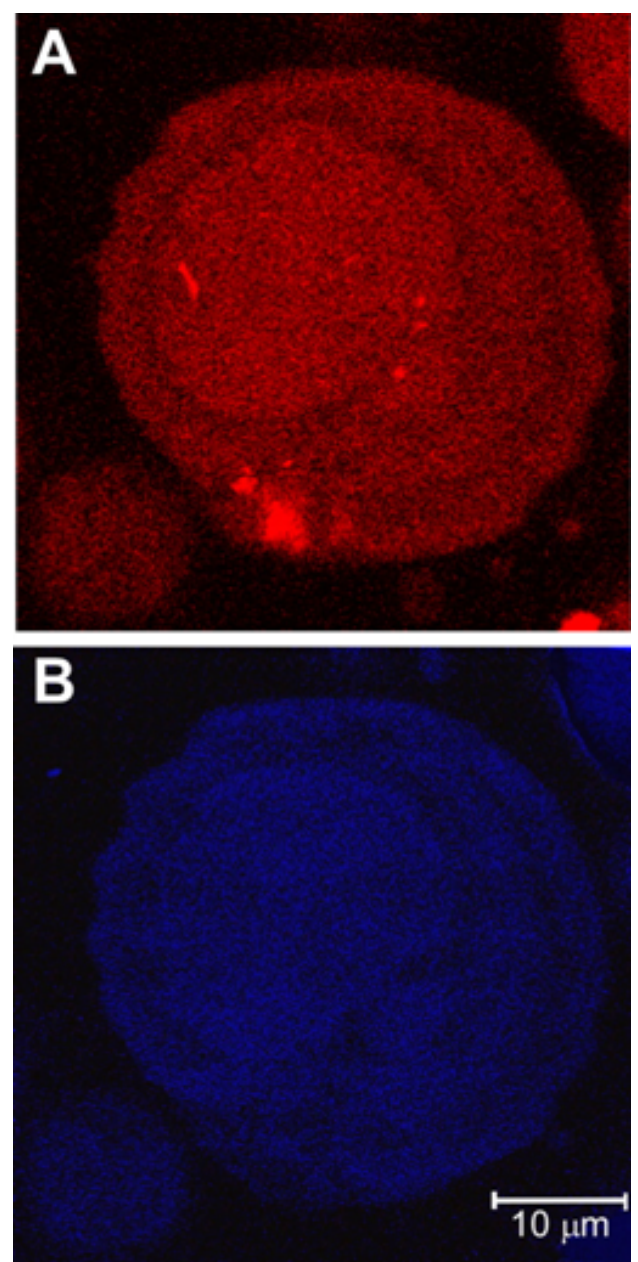

Figure 7.3: Reconstructed giant liposome under confocal microscopy. $A$ : fluorescence microscopy images of KcsA clusters in a confocal cross-section of a giant liposome containing Alexa 546-labeled KcsA. Large and highly fluorescent array-like protein complexes of variable sizes are seen over a more homogeneous protein fluorescence background. In contrast to the marked heterogeneity in the distribution of the KcsA protein from above, $B$ shows that the fluorescence of a phospholipid probe (NBD-DMPE) recorded in the same liposome is distributed much more homogeneously. Adapted from Molina et al. [127]. 
multiple-channel openings in the recordings. Furthermore, a common sensing of the fluctuation of an exogenous messenger was also ruled out as the cooperativity was observed even in the absence of important messenger such as cAMP, PIP2 and $\mathrm{Ca}^{2+}$. They suggested allosteric communication between channels as a simpler hypothesis (Dekker and Yellen [48]).

The hypothesis of allosteric communication would easily be adapted for other channels that were shown to act cooperatively. Therefore, I will mention this aspect once again in the end of this chapter.

\subsection{2 $\mathrm{Ca}^{2+}$ channels}

The early studies on $\mathrm{Ca}^{2+}$ release channels (or ryanodine receptors) of the endoplasmic and sarcoplasmic reticulum characterized the structure and kinetics of this channel (Saito et al. [155]; Franzini-Armstrong and Protasi [64]). In one of those studies, the ryanodine receptors were shown to form clusters using electron micrography (Saito et al. [155]). As mentioned previously, the clustering of membrane proteins may leas to functional inter-protein coupling (Huang [87]). Therefore, such interaction could also be expected from ryanodine receptors. This prediction was confirmed by another study that came ten years later. In $\approx 10 \%$ of the experiments performed in the study, RyR1 channels exhibited single channel-like openings with two-fold (8 pA) of single channel current amplitudes (4 pA) (Marx et al. [120]). This familiar phenomenon was statistically analyzed again using the peak amplitude histograms, which failed to show binomial distribution (see section 7.1.1). This and the results of previous studies that confirmed the single channel current measurement of 4 pA approved the suggestion that $\approx 10 \%$ of ryanodine receptors act as functionally coupled. It was also intriguing that the electron micrographs of purified RyR1 homotetramers, that were obtained in the previous study (Saito et al. [155]), showed that $\approx 10 \%$ of the structures are physically connected to form contacting pairs.

\subsection{3 $\mathrm{Na}^{+}$channels}

The only study that showed cooperative activity for $\mathrm{Na}_{V}$ channels was performed on cardiomyocytes of rat and rabbit (Undrovinas et al. [179]). The study indicated that lysophosphatidylcholine (LPC)-treated $\mathrm{Na}_{V}$ channels exhibit opening patterns that can only be explained by inter-channel coupling.

The results of the study indicated that LPC reduces the opening probability and induces sustained open channel activity at depolarized potentials. LPC modification, that caused a shift in activation, did not affect single channel conductance or reversal 


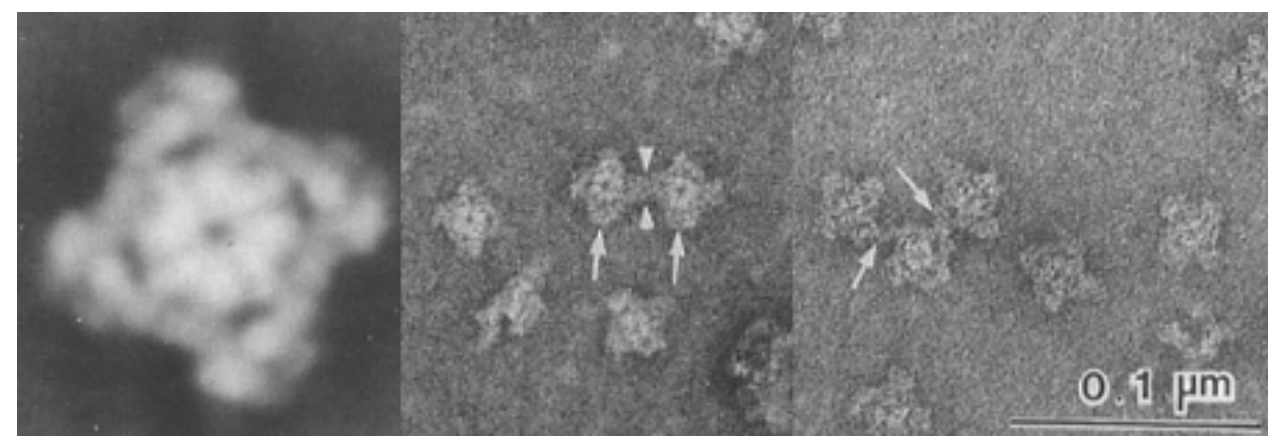

Figure 7.4: Ryanodine receptor clustering observed by thin-layer electron microscopy. Left: The computer averaged top view of the ryanodine receptors, obtained by averaging 240 negatively stained images. Middle and Right: After negative staining, the purified receptor feet structures can be observed to connect. In the middle, two cross-like structures connect via the legs of the cross, and the distance between the white arrowheads is $12 \mathrm{~nm}$. On the right, the square-like structures associate approximately at the corners, giving an overlap of $8 \mathrm{~nm}$ (between white arrows). Adapted from Saito et al. [155].

potential. The activation threshold shifted towards more negative potentials and the midpoint of Boltzmanian conductance transforms shifted by $26 \mathrm{mV}$ in cell-attached patch-clamp and by $17 \mathrm{mV}$ in inside-out patch-clamp. Most importantly, multi-level openings were observed as superimposed opening of one channel on another. Synchronous activity was evident in single channel current traces.

The authors also utilized the peak amplitude histogram to confirm the coupling of $\mathrm{Na}^{+}$channels. Non-binomial histograms provided further evidence for stochastically non-independent channel gating.

Undrovinas et al. [179] suggested some possible mechanisms that can be responsible for LPC-modified gating, such as :

- Changes in the membrane fluidity,

- Changes in the lipid protein interaction,

- The activation of mechanisms, that are activated infrequently in non-LPCtreated cells (e.g. increase in cAMP due to increasing levels of LPC at heart tissue (Ahumada et al. [1])),

- Disruption of cytoskeleton due to LPC-induced ischemia, which leads to further changes in the membrane structure (Cantiello et al. [31]). 


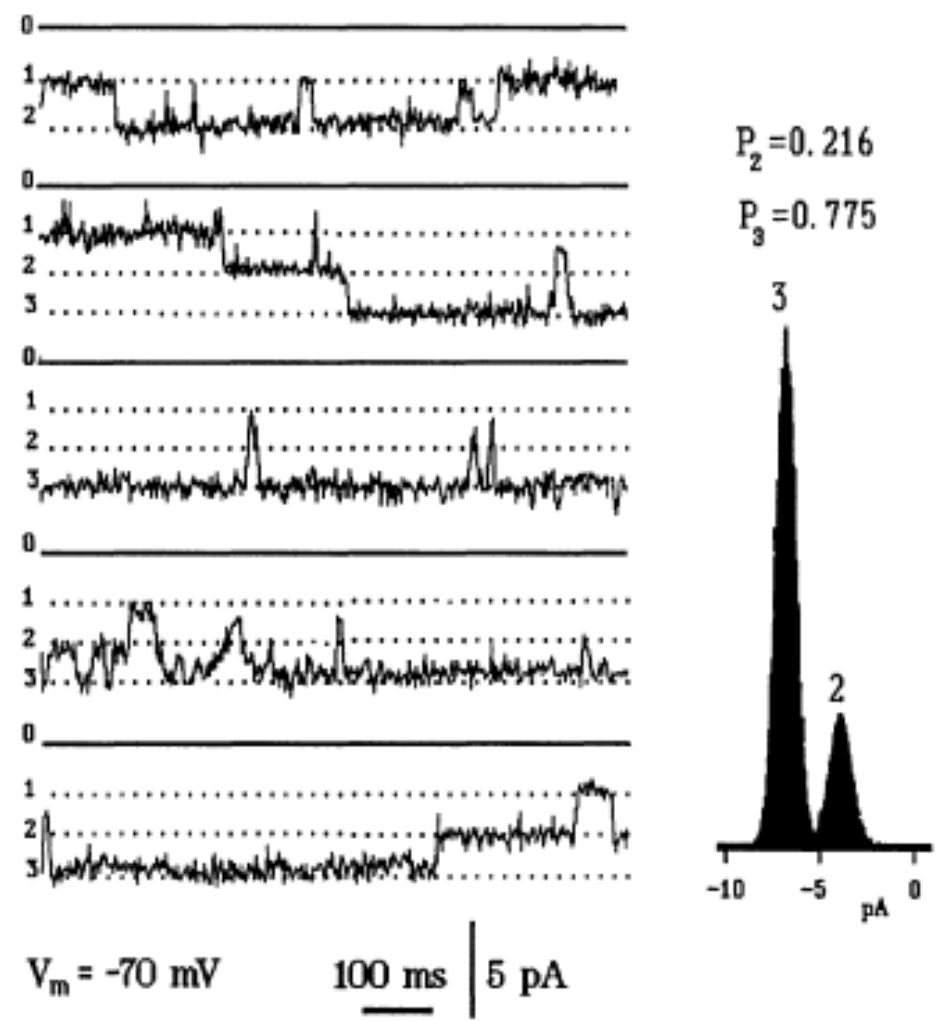

Figure 7.5: Amplitude analysis of LPC-modified $\mathrm{Na}^{+}$channel current and examples of channel activities from an inside-out patch at a membrane potential $\mathbf{V}_{\text {mem }}=\mathbf{- 7 0} \mathbf{~} \mathbf{~ V}$. The numbers 1,2,3 at the left each dotted line indicate the single, double or triple unitary conductance levels. The channel current amplitude distribution histograms are given on the right. At $\mathrm{V}_{m e m}=-70 \mathrm{mV}$, the triple the single channel current amplitude openings are more frequent than double amplitude openings. Single amplitude events were observed rarely. The openings and closings to double and triple levels were synchronized. The probabilities for a triple amplitude opening $\left(P_{3}\right)$ and a double amplitude opening $\left(P_{2}\right)$ are given above the histogram. Adapted from Undrovinas et al. [179]. 


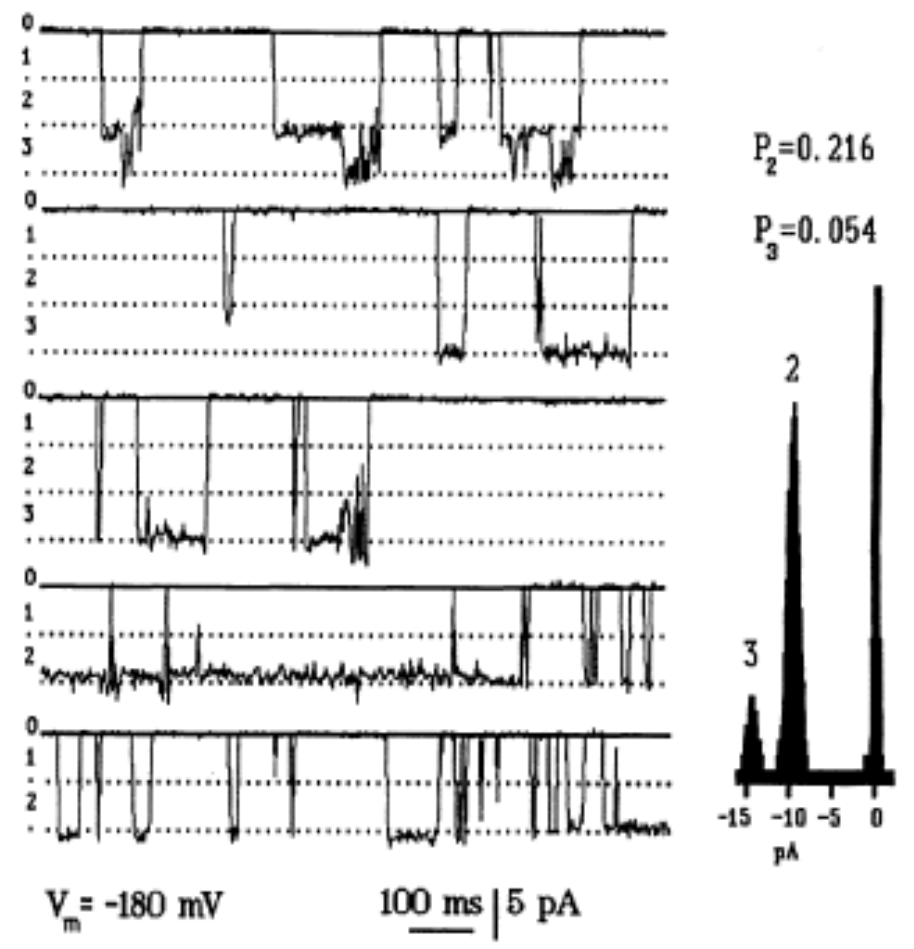

Figure 7.6: Amplitude analysis of LPC-modified $\mathrm{Na}^{+}$channel current and examples of channel activities from an inside-out patch at a membrane potential $\mathbf{V}_{\text {mem }}=\mathbf{- 8 0} \mathbf{m V}$. The numbers 1,2,3 at the left each dotted line indicate the single, double or triple unitary conductance levels. The channel current amplitude distribution histograms are given on the right. At $\mathrm{V}_{\text {mem }}=-180 \mathrm{mV}$, the triple the single channel current amplitude openings are less frequent than double amplitude openings. Single amplitude events were observed rarely. The openings and closings to double and triple levels were synchronized. The probabilities for a triple amplitude opening $\left(P_{3}\right)$ and a double amplitude opening $\left(P_{2}\right)$ are given above the histogram. The zero current peak of the histogram was truncated at 6000 counts. Adapted from Undrovinas et al. [179]. 
The last mechanism was also observed for the clustering of ryanodine receptors by freeze-fracture method (Post et al. [142]) and, although seemingly contradictory, it can account for some levels of clustering. Therefore, it will be mentioned also in 7.1.4.

\subsubsection{How do the neighboring channels couple?}

Although some biological evidence about the cooperative activity of ionic channels are provided, details of the underlying molecular mechanisms for such phenomena are still unknown. However, to have synchronous activity in a population of ionic channels, it is clear that the gating state changes of one channel must be transmitted to the neighboring channels in some way. For this type of allosteric communication between ionic channels, several mechanisms are possible candidates :

1. A protein-protein interface between channels, i.e. due to dimer, trimer or tetramer formations (Neumcke and Stämpfli [134]; Angelides and Nutter [8]; Almers and Stirling [4]),

2. Reconciliation by an adaptor protein,

3. Allosteric changes in a linking cytoskeleton (Post et al. [142]; Undrovinas et al. [179]; Grubb and Burrone [72]),

4. Interactions between subdomains of channel proteins, e.g. that are the binding sites of cytosolic secondary messengers like cAMP, $\mathrm{Ca}^{2+}$ or PIP2 (Dekker and Yellen [48]).

Other than allosteric communication, the coupling through the changes in the local electric field is yet another possibility. The excess charges that flow through the open channels may be also acting as cytosolic modulators for the neighboring channels up to certain concentrations. Furthermore, the voltage-dependent or -independent elastic changes of the lipid membrane may also lead to conformational changes in the ionic channels, that may contribute to inter-channel coupling (Ursell et al. [180]; Silberberg and Magleby [167]).

Taking the specific molecular structure of AIS into account, an allosteric communication through linking cytoskeletal structures seems as a plausible mechanism to coordinate cooperative $\mathrm{Na}_{V}$ channel gating in this region. As mentioned in Part I, the cytoskeleton of AIS, especially AnkyrinG and $\beta \mathrm{IV}$-spectrin, are shown to be essential in the recruitment and localization of specific $\mathrm{Na}_{V}$ channels. However, further investigation is required to prove inter-channel coupling in AIS experimentally. 


\subsection{Functional relevance to neural encoding}

Voltage-gated $\mathrm{Na}^{+}$channels play the major role in the onset and the upstroke of the action potentials. Therefore, the cooperative gating of these channels would results in significant changes at the action potential initiation timescale. It was shown that the onset rapidness could be an indirect measure of cut-off frequency (Fourcaud-Trocme et al. [63]). Furthermore, the recent in vitro studies provided information about high cut-off frequencies of real neurons (Köndgen et al. [100]; Boucsein et al. [20]) and a recent theoretical work reported that smaller timescales at the AP onset help to reproduce such high cut-off frequencies (Wei and Wolf [185]). Combining these results, it can be concluded that very rapid onsets, such as the ones observed in cortical neurons (Naundorf et al. [130],[132]), may lead to high cut-off frequencies in physiological conditions. It is also reported that cooperative gating kinetics, when implemented in classical Hodgkin-Huxley formulations, can indeed reproduce two most important effects of the cortical neuron dynamics: rapid AP onsets and threshold variability (Huang et al. [89]). Therefore, it is alluring to test this hypothesis on a more realistic neuron model, and i.e. implementing the dynamics in AIS. 
102 Chapter 7: Cooperative gating of voltage-gated ionic channels 


\section{8}

\section{Single-Compartment Conductance-Based Cooperative Channel Gating (SC-COOP) Model}

The functional and the dynamical consequences of the cooperative channel gating was first examined using spherical conductance-based single-compartment neuron model.

It was assumed that only a fraction $p$ of $\mathrm{Na}^{+}$channels exhibit the cooperative channel gating. The inter-channel coupling strength $(K J)$ and cooperativity percentage $(p)$ was introduced in the model construction, such that it could be varied from statistical independence ( $p=0$ and/or $K J=0)$ to very strong coupling ( $K J$ $>300 \mathrm{mV})$ in small $(p<30 \%)$ or large $(p=30 \%-50 \%)$ fractions of channel population.

The SC-COOP model was especially useful for the characterization of the activation kinetics and of the impact of the cooperativity on action potential waveforms and response properties for a wide range of cooperativity fraction and coupling strengths. The predictions of the model supported the hypothesis that introducing cooperative gating kinetics instead of the canonical gating kinetics reproduce the rapid onsets observed in cortical neurons (Naundorf et al. [130], [132]), with increasing steepness as the coupling strength and coupling fraction increase. For a small range of KJ and p, the model exhibited the biphasic action potential waveform, which is often observed in the neurons of central nervous system (Eccles et al. [53]; Bean [15]). This finding points out that steep action potential onsets and biphasic action potential waveforms might be triggered by the simultaneous opening of a group of $\mathrm{Na}^{+}$channels.

\subsection{Model Methods}

The SC-COOP model was implemented using $\mathrm{C}++$ in Linux environment. The implicit backward Euler integration method was used with a $10 \mu$ s time step. The membrane capacitance $\left(\mathrm{C}_{m}\right)$ was $1 \mu \mathrm{F} / \mathrm{cm}^{2}$. The radius of the sphere was $1 \mu \mathrm{m}$, if 


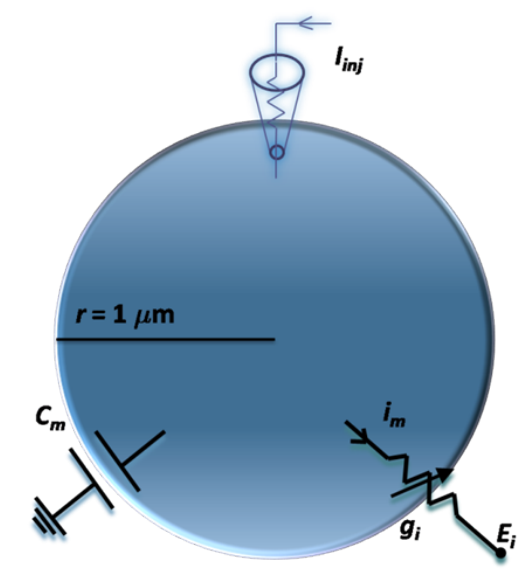

Figure 8.1: The spherical single compartment model

not mentioned otherwise. Therefore, the surface area was $12.6 \mu \mathrm{m}^{2}$. The membrane resistance $\left(\mathrm{R}_{m}\right)$ was $10 \mathrm{~K} \Omega . \mathrm{cm}^{2}$ and the membrane time constant was estimated as $10 \mu \mathrm{s}$.

\subsubsection{Conductance-Based Model}

We used a slightly modified version of the conductance-based that was proposed by Wang and Buzsàki [183] (WB model) as described in section 6.1.6. This HodgkinHuxley type model served as the backbone of our construction as we implemented the cooperative sodium channel gating (Huang et al. [89]).

In WB model, $\mathrm{Na}^{+}$channels respond to a voltage step by instantaneous activation. This model exhibits type-I excitability and therefore, it has been employed in various theoretical studies to explore the dynamical response properties of cortical neurons (Wang and Buzsàki [183]; Fourcaud-Trocme and Brunel [62]; Naundorf et al. [131]; Fourcaud-Trocme et al. [63]).

\subsubsection{Channel Gating Kinetics I : Statistically Independent Gating}

A widely accepted explanation for the gating kinetics of the voltage-gated ionic channels was provided by Alan Lloyd Hodgkin and Andrew Fiedling Huxley in 1952 after their electrophysiology experiments on the giant axon of squid. One essential assumption of their model was that the activity of individual channels is independent from its neighboring channels. 


\begin{tabular}{|c||c|c|}
\hline Channel Type & Gating Particle & V-dependence \\
\hline \hline $\mathrm{Na}^{+}$channel & $\mathrm{m}$ & $\alpha_{m}=\frac{0.1(V+35)}{1-\exp [-0.1(V+35)]}$ \\
& & $\beta_{m}=4 \exp \left(\frac{-(V+60)}{18}\right)$ \\
\hline & $\mathrm{h}$ & $\alpha_{h}=0.35 \exp \left(\frac{-(V+58)}{20}\right)$ \\
& $\mathrm{\beta}$ & $\frac{5}{1+\exp [-0.1(V+28)]}$ \\
\hline $\mathrm{K}^{+}$channel & $\mathrm{n}$ & $\alpha_{n}=\frac{0.05(V+34)}{1-\exp [-0.1(V+34)]}$ \\
& & $\beta_{n}=0.625 \exp \left(\frac{-(V+44)}{80}\right)$ \\
\hline \hline
\end{tabular}

Table 8.1: The voltage dependence of $\mathrm{Na}^{+}$and $\mathrm{K}^{+}$type channels in Wang-Buzsaki model (Wang and Buzsàki [183]).

The channel gating kinetics for a gating particle $z$ is modelled by the equation

$$
\tau_{z} \frac{d z}{d t}=z_{\infty}-z(t)
$$

where $\tau_{z}$ is the time constant and $z_{\infty}$ is the steady-state opening probability (or the limiting value).

Each gating particle is assumed to switch between open and close states with an opening rate, $\alpha(V)$, and a closing rate, $\beta(V)$. The dynamics of the time constant and the steady-state probability is governed by the equations utilizing these rates, such that

$$
\begin{aligned}
\tau_{z} & =\frac{\Phi}{\alpha(V)+\beta(V)}, \\
z_{\infty} & =\frac{\alpha(V)}{\alpha(V)+\beta(V)},
\end{aligned}
$$

where $\Phi$ is a constant. To maintain a maximum activation time constant of $50 \mathrm{~s}$, which is suggested by the sodium current measurements on cortical neurons (Baranauskas and Martina [13]), $\Phi$ was set to 0.1.

The activation of $\mathrm{Na}^{+}$channel is instantaneous, therefore $m$ is substituted by the steady-state function, $m_{\infty}$.

The opening and closing rates are dependent only on local membrane potential, $\mathrm{V}$, as given in Table 8.1. 


\subsubsection{Channel Gating Kinetics II : Cooperative Gating}

The cooperative gating model assumes that a single channel is coupled to $K$ neighbor channels, such that the opening of one channel increases the opening probability of its neighbors. It might also be assumed that this cooperative activity will have similar effects with a voltage shift in the membrane potential. Therefore, a unit coupling strength in $\mathrm{mV}, J$, can be defined such that it represents a voltage shift in the membrane potential that results in the same amount of increase in the opening probability of a single channel. Then, cooperativity can be implemented in the classical model, by giving the voltage shift

$$
V_{\text {shift }}=K J\left(m_{\text {coop }}\right)^{x} h_{\text {coop }}
$$

and adding it in the canonical equations,

$$
\begin{gathered}
\tau_{m}^{\text {coop }} \frac{d m_{\text {coop }}}{d t}=m_{\infty}^{\text {coop }}-m_{\text {coop }}(t), \\
\tau_{m}^{\text {coop }}(V)=\tau_{m}\left(V+V_{\text {shift }}\right), \\
m_{\infty}^{\text {coop }}(V)=m\left(V+V_{\text {shift }}\right),
\end{gathered}
$$

and

$$
\begin{gathered}
\tau_{h}^{\text {coop }} \frac{d h_{\text {coop }}}{d t}=h_{\infty}^{\text {coop }}-h_{\text {coop }}(t), \\
\tau_{h}^{\text {coop }}(V)=\tau_{h}\left(V+V_{\text {shift }}\right), \\
h_{\infty}^{\text {coop }}(V)=h\left(V+V_{\text {shift }}\right) .
\end{gathered}
$$

The opening of individual channels are modeled as a Markov process; and the equations 8.5 and 8.8 represent the mean field approximation of cooperative channel gating among a coupled population (Naundorf et al. [132]).

(Note : From now on, KJ will be referred together as the coupling strength for simplicity.)

It is also assumed that only some fraction $p$ of the channel population in a certain area couple. Therefore, the cooperative $g_{N a}$ can be rewritten as

$$
g_{N a}=\left[(1-p) m^{3} h+p m_{\text {coop }}^{x} h\right] \bar{g}_{N a}
$$

and $\mathrm{p}$ represents the percentage of coupling channels in the population. 
Note that, $x=1$ for a part of the voltage clamp simulations (Figure 8.3) and $x=3$ for the rest of the simulations. We could simplify our analytical tests by using $x=1$ as it was suggested by in vitro recordings of $\mathrm{Na}^{+}$currents in cortical neurons (Baranauskas and Martina [13]).

\subsubsection{Current Injection}

The current templates, that mimic the in vivo synaptic currents, were generated in $\mathrm{C}++$ environment as embedded in the source code of the model. The noisy current $\left(I_{\text {noisy }}\right)$ was generated as a realization of an Ornstein-Uhlenbeck stochastic process with zero mean and variance $\sigma_{I}^{2}$ to mimic the synaptic noisy input as following :

$$
\tau_{I} I_{n o i s y}=-I_{n o i s y}+\kappa \zeta_{t}
$$

where $\zeta_{t}$ is a random variable drawn at every time step from a Gaussian distribution with a zero mean, called white noise; $\kappa=X / \Delta t$ and $\zeta_{t}=\xi_{t} / \Delta t$. Integrating the deterministic part gives us

$$
I_{\text {noisy }}(t+\Delta t)=I_{n o i s y}(t) \exp \left(-\frac{\Delta t}{\tau_{I}}\right)+X \xi_{t}
$$

and $\mathrm{X}$ can be set as

$$
X=\sigma_{I} \sqrt{1-\exp \left(\frac{2 \Delta t}{\tau_{I}}\right)}
$$

where $\sigma_{I}$ is the variance of $I_{n o i s y}$.

The value of $\tau_{I}$ corresponds the decay time constants of synaptic currents. In a range of 5-20 ms fast synaptic currents such as AMPA- and GABA $_{A}$-mediated currents. The current was injected at the middle compartment of the soma (e.g. $5^{\text {th }}$ compartment).

\subsubsection{Data analysis}

Data were obtained from the simulation as either potential traces, spike times or both. Data analysis were performed in MATLAB v7.0.0 and v7.2.0 using custom-written functions and procedures. 


\section{Measuring Onset Rapidness}

Comparing the onset rapidness of action potentials that are generated by different mechanisms (i.e. canonical and cooperative channel gating kinetics) demands a solid way of measuring these values. First of all, a threshold for the onset must be set. Although there are several methods to describe a threshold, $\dot{V}\left(t_{i}\right)=V / s$ is chosen to define an onset. Then, the slope of the phase plot ( $V$ vs $\dot{V})$ at $\dot{V}\left(t_{i}\right)$ is measured to obtain the onset rapidness, $\Gamma$.

\subsection{Activation Kinetics of Cooperatively Gating Sodium Channels}

The steady state solution of $m_{\infty}^{\text {coop }}$ is obtained from the intersection points of two curves

$$
\begin{gathered}
y=m ; \\
y=f(m)=\frac{1}{1+\exp \left[-\frac{V+\lambda_{a} m+V_{1 / 2}}{k_{A}}\right]},
\end{gathered}
$$

where $\lambda_{a}=\mathrm{KJH}_{0}$. The details of this solution will be given in Appendix A of the chapter. There is only one intersection point, $m_{0}$, that can be obtained from

$$
1-\lambda m_{0}+\lambda m_{0}^{2}=0
$$

where

$$
\lambda=\frac{K J H_{0}}{k_{A}} .
$$

The solution has real roots only for

$$
\begin{aligned}
\Delta & =\lambda^{2}-4 \lambda \\
\lambda \geq &
\end{aligned}
$$

Therefore, there will be a finite jump in the collective activation curve only when

$$
K J \geq \frac{4 k_{A}}{H_{0}} .
$$

To see the impact of cooperativity on the activation of $\mathrm{Na}^{+}$channels, $\mathrm{m}_{\text {inf }}^{3}$ and $\tau_{m}$ were plotted against the membrane potential and also against each other (Figure 8.2). The step-function-like activation curve for strong coupling of channels ( $K J$ $=400 \mathrm{mV}$ ) implies that the opening of these channel occur instantly after crossing 


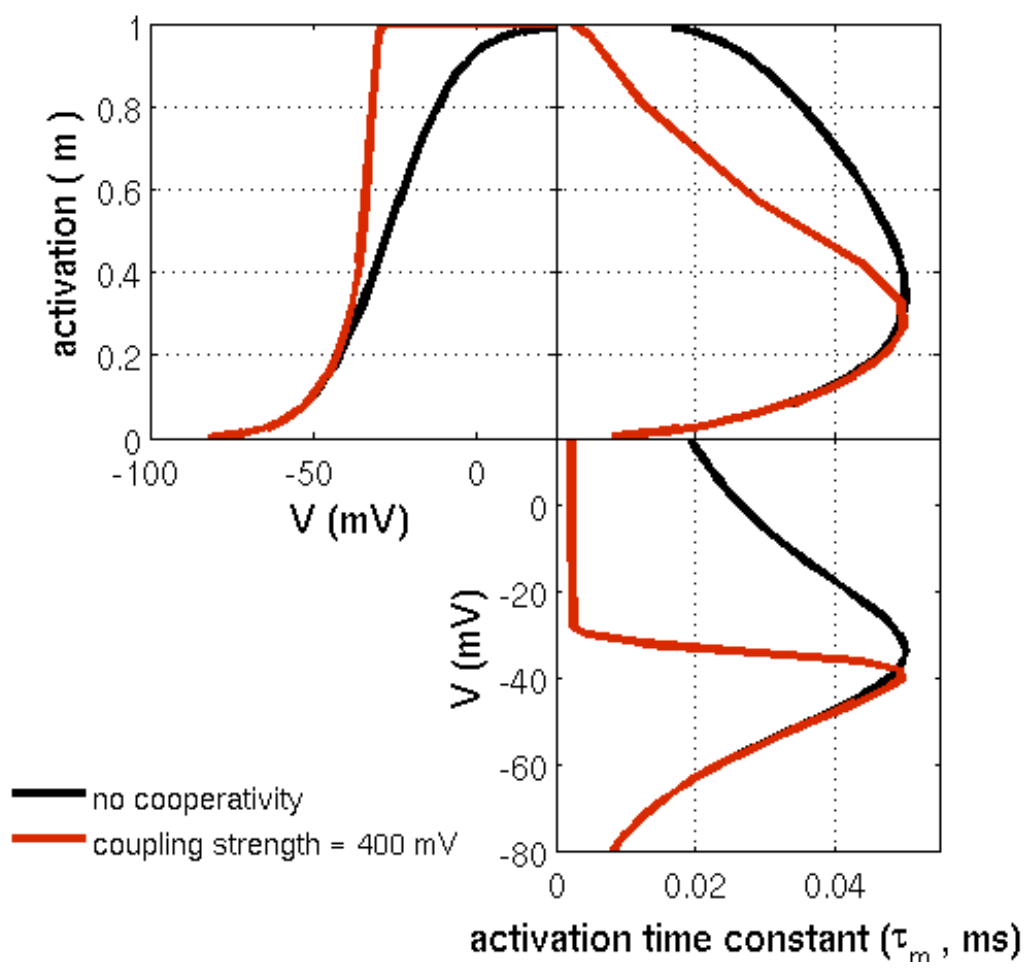

Figure 8.2: The activation kinetics of $\mathrm{Na}^{+}$channels in cooperative and noncooperative model. The red line represents the cooperative model with strong coupling $(K J=400 \mathrm{mV})$ and the black line represents the non-cooperative model

the threshold potential, almost as an all-or-none event. On the other hand, noncooperative activation has a sigmoidal dependence on the voltage. Activation time constants for cooperative and non-cooperative models display the same pattern : For potential higher than $-20 \mathrm{mV}$, strong coupling results in almost ten-fold smaller activation time constant compared to the non-cooperative model, which also implies an abrupt opening of the channels.

The collective activation curves for different $\lambda$ values are depicted in Figure 8.3. The slopes of the sigmoidal activation curves increase by increasing $\lambda$. After the critical coupling strength, $\left(\lambda^{*}=4\right)$, the curve exhibits a discontinuity at critical threshold voltage, $V_{t h}^{*}$. This behavior stems from an abrupt opening at threshold potential. The threshold voltage shifts to more negative values $\left(V_{t h}<V_{t h}^{*}\right)$ for stronger coupling $(\lambda$ $\left.>\lambda^{*}\right)$ and the activation curve approaches to a step function. This implies a jump from a state that almost all the channels are closed $\left(m_{\text {coop }} \cong 0\right)$ to a state that almost all the channels are open $\left(m_{\text {coop }} \cong 1\right)$. 

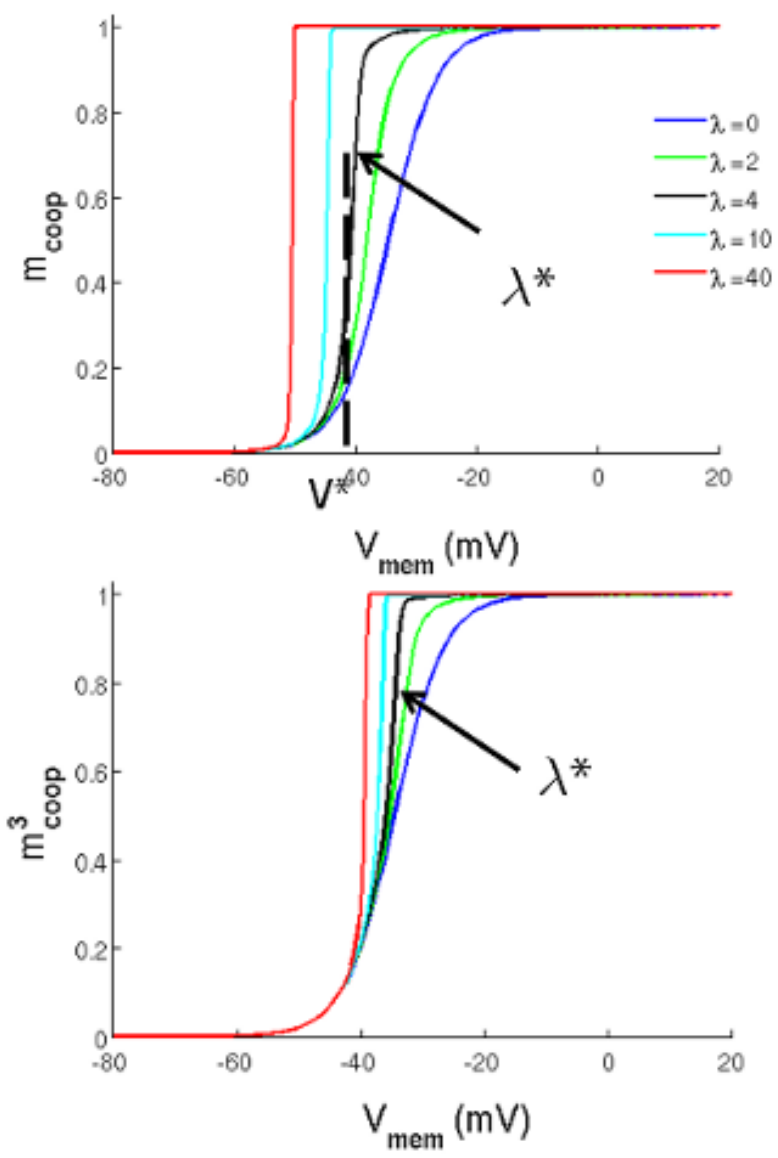

Figure 8.3: The collective activation curves for $\mathrm{x}=1$ (upper figure) and $\mathrm{x}=$ 3 (lower figure). The color ligand is also given with the upper figure. The arrows in both figures indicate the curves for critical coupling strength $\left(\lambda^{*}\right)$. The dashed line in the upper figure corresponds to the critical voltage threshold, which is $V_{1 / 2}$ of the curve for $\lambda^{*}$. 
The opening probability of finding $\mathrm{Na}_{V}$ channels with cooperative gating kinetic at a time $t$ is calculated as $\mathrm{P}_{\text {open }}=m_{\text {coop }}^{x} h$. At $\mathrm{V}_{\text {mem }}<V_{t h}^{*}$, a smaller percentage of $\mathrm{Na}_{V}$ channels open more slowly compared to $\mathrm{V}_{m e m}>V_{t h}^{*}$. However, they also inactivate more slowly at lower voltage values therefore the duration that they remain open lasts longer. The effective coupling strength strongly affects $V_{t h}^{*}$ and the duration that the channels remain open. $V_{t h}^{*}$ shifts to more negative values as $\lambda$ increases and $\mathrm{P}_{\text {open }}$ decrease more slowly, meaning that the number of channels that remain open decreases more slowly.

\subsection{Action Potential Waveforms}

The first few $\mu$ s of an action potential give us an insight about the inherent action potential generation mechanisms of a neuron. It is almost impossible to have a quantitative measure of this very small scale onset using a classical V-t plot. On the other hand, the graphs of $\dot{V}$ vs $\mathrm{V}$, or the phase plots, are useful tools of measuring action potential onsets because they clearly depict the rate of potential change at a certain point membrane potential. A more detailed explanation of phase plots is given in Figure 8.4.

\subsubsection{Impact of Cooperativity on Action Potential Waveform and Onset Rapidness}

In response to a constant current injection, the action potential waveforms $\mathrm{f}$ the model are scanned in a 2-D parameter space of cooperativity percentage $p$ and coupling strength $K J$. The resulting phase plots were very sensitive to both of the parameter (Figures 8.5 and8.6). In response to a large fraction of this parameter space, the rising phase of action potentials were monophasic, however, biphasic rising phases were also observed. Both regimes were highly sensitive to cooperative activity (Figure 8.5 and 8.6).

For weak inter-channel coupling $(K J<200 \mathrm{mV})$, action potentials exhibit a gradual rising phase. For strong coupling in a small percentage of the channel population, the action potentials exhibit biphasic rising with an initial phase of cooperative channel activation followed by a slower rising phase with canonical channel activation. This type of action potentials are frequently observed in neurons of mammalian central nervous system (Eccles et al. [53]; Bean [15]). On the other hand, strong coupling in a large percentage of channel population induces action potentials with steep and monophasic rising phases (Figures 8.5 and8.6).

Onset rapidness, $\Gamma$, increases monotonically with increasing coupling strength (Figure 8.7). Action potentials of cortical neurons under physiological conditions 
112 Chapter 8: Single-Compartment Conductance-Based Cooperative Channel Gating (SC-COOP) Model
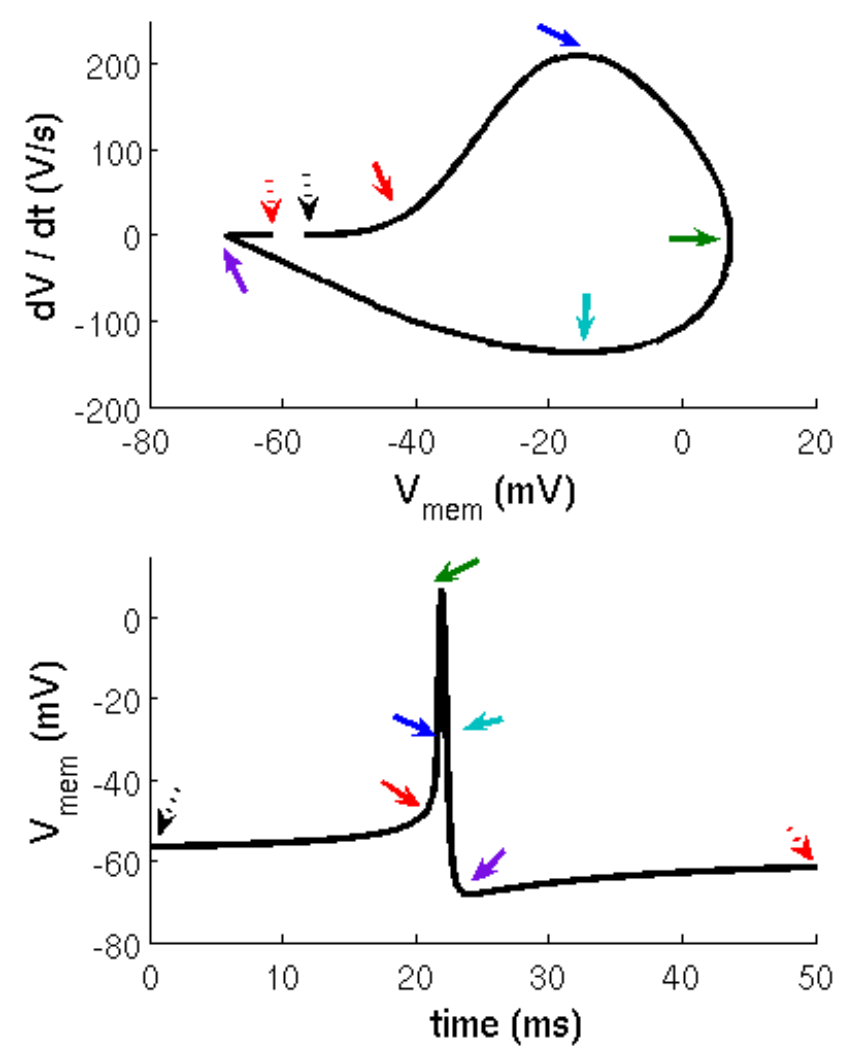

Figure 8.4: The upper figure is the phase-plot representation of the action potential in the lower figure. Same color and shaped arrows indicate same time points. 

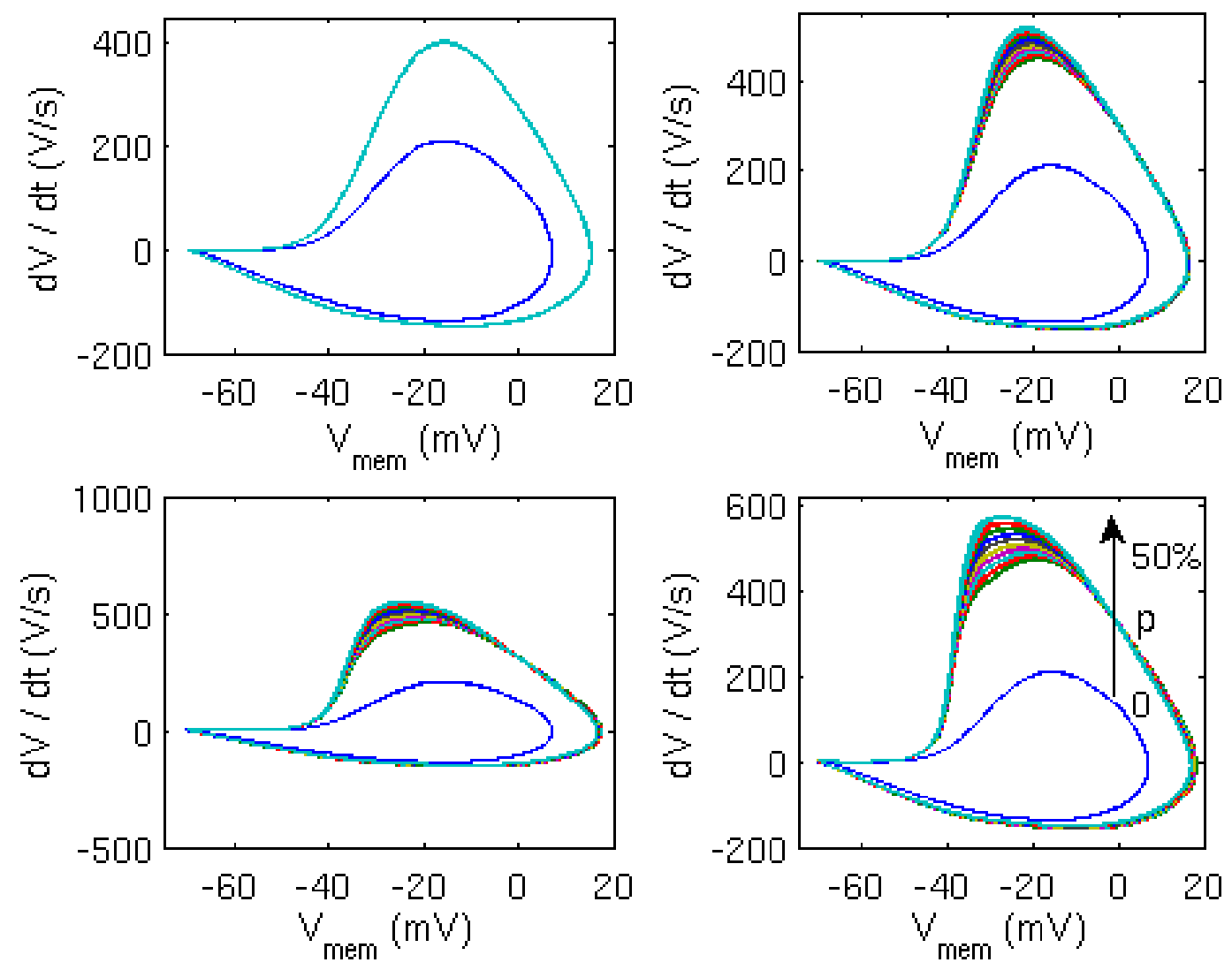

Figure 8.5: Phase plots for different coupling strengths and coupling percentages. A) $\mathrm{KJ}=20 \mathrm{mV}$, B) $\mathrm{KJ}=100 \mathrm{mV}$, C) $\mathrm{KJ}=150 \mathrm{mV}$, D) $\mathrm{KJ}=200 \mathrm{mV}$. $p$ values were altered for each trace from 0 (blue) to $50 \%$ (uppermost light blue). The $p$ values increase by $5 \%$ for each trace and the peak of $\dot{V}$ vs $\mathrm{V}$ increase by increasing $\mathrm{p}$ values (as shown with the arrow on the right-bottom). 

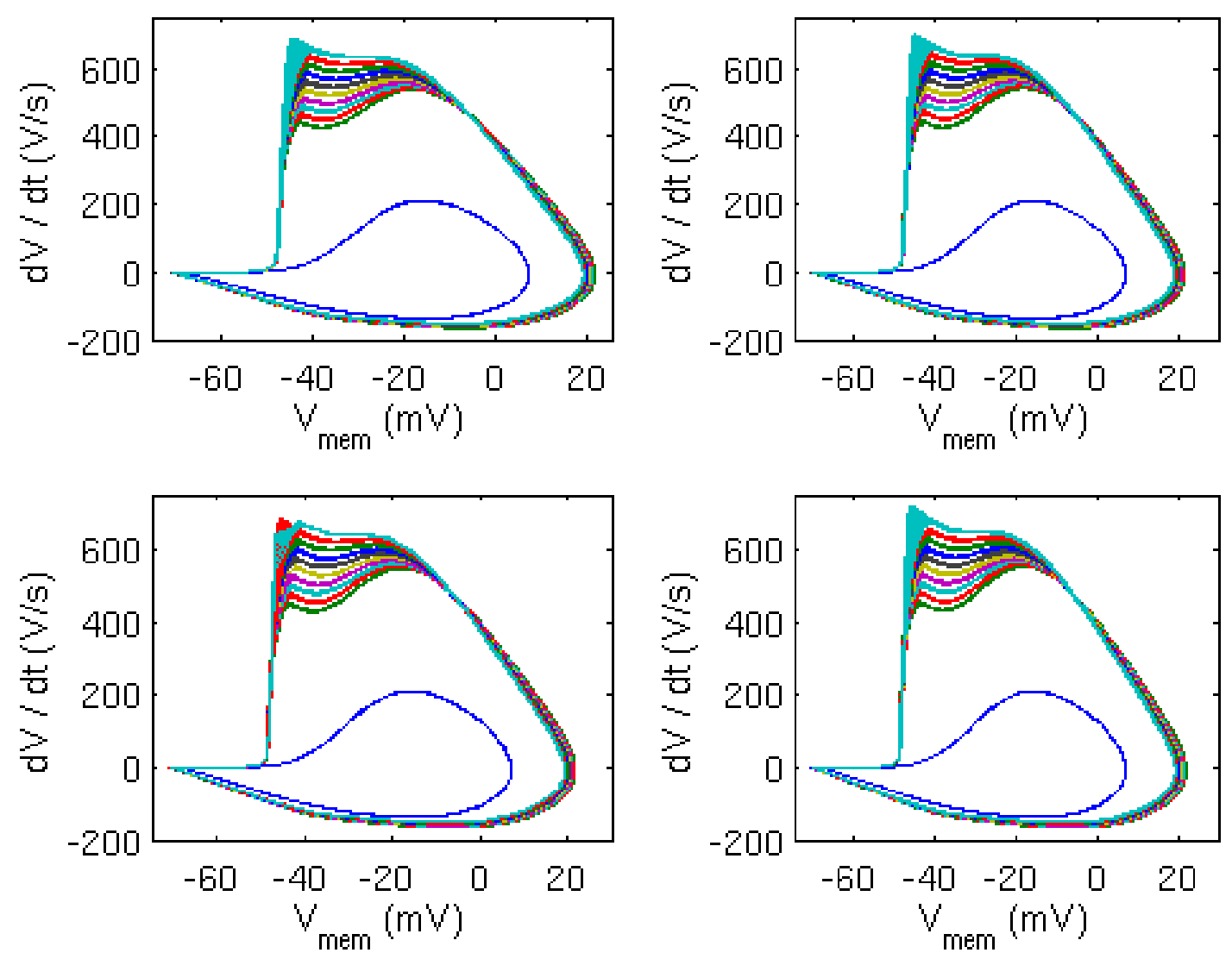

Figure 8.6: Phase plots for different coupling strengths and coupling percentages. A) $\mathrm{KJ}=650 \mathrm{mV}$, B) $\mathrm{KJ}=700 \mathrm{mV}$, C) $\mathrm{KJ}=750 \mathrm{mV}$, D) $\mathrm{KJ}=800 \mathrm{mV}$. $p$ values were altered for each trace from 0 (blue) to $50 \%$ (uppermost light blue). The $p$ values increase by $5 \%$ for each trace and the peak of $\dot{V}$ vs $\mathrm{V}$ increase by increasing p values (as shown in Figure 8.5). 


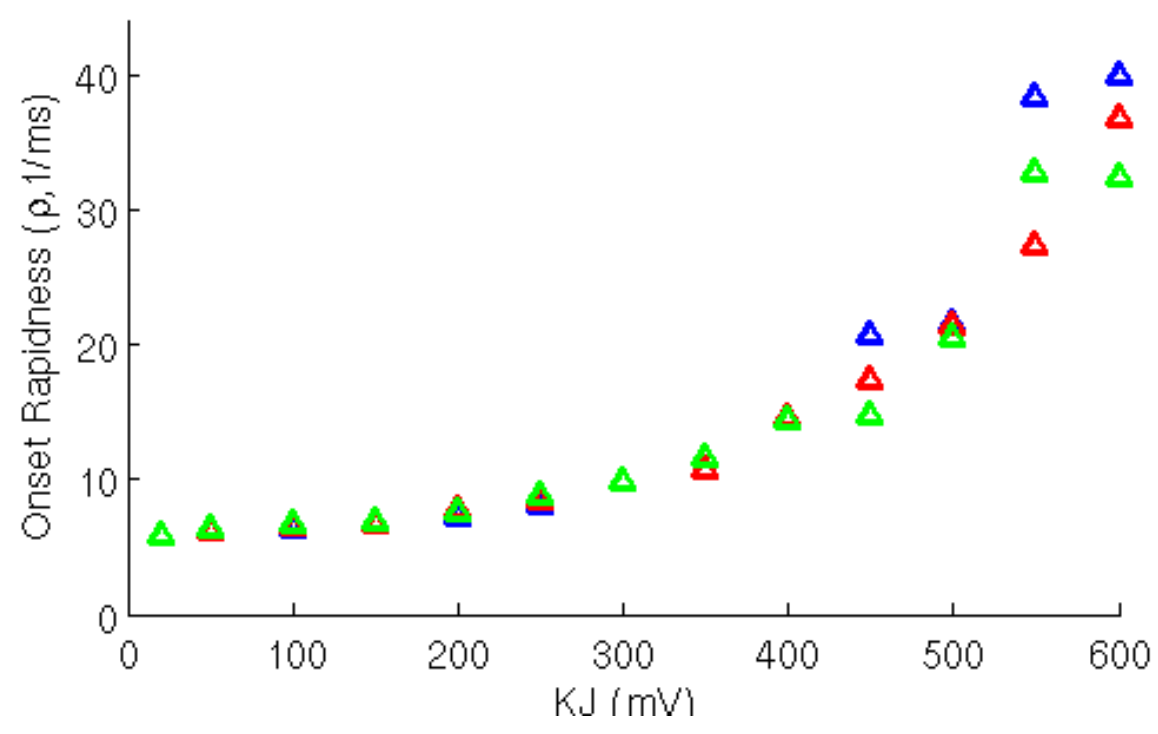

Figure 8.7: The change in onset rapidness $(\Gamma)$ with increasing coupling strength for $\mathrm{p}=\mathbf{1 0} \%$ (blue), $\mathrm{p}=\mathbf{2 0 \%}$ (red) and $\mathrm{p}=\mathbf{3 0} \%$ (green). $\Gamma$ is not affected with the coupling percentage for $\mathrm{KJ}<400 \mathrm{mV}$ and show deviations for higher values of KJ. On the other hand, $\Gamma$ increases for $\mathrm{KJ}>150 \mathrm{mV}$. This finding suggests that the coupling strength is a more critical parameter for onset rapidness measurements compared to the coupling percentage.

exhibit a minimum $\Gamma$ of 20 1/ms. Total coupling strength to obtain such an action potential onset in our model is at least $400-450 \mathrm{mV}$. In other words, if it is assumed that a channel is coupled with 10 neighboring channels, opening of each channel would be equivalent to a voltage shift of approximately $40 \mathrm{mV}$. Such strong interchannel coupling would trigger highly synchronized channel openings and the coupled channels would behave as a single unit. In the previous studies that provided evidence of inter-channel coupling, exactly this type of highly synchronized channel activity was reported (Marx et al. [120]; Undrovinas et al. [179]; Molina et al. [127]).

\subsection{Impact of Current Statistics on Non-Cooperative Model}

The properties of the injected current may greatly affect the properties of neuronal response. Therefore, the input parameters $\sigma_{I}$ and $\tau_{I}$ of noisy current were altered in successive trials before testing the model further with cooperative gating kinetics, and the impact on characteristics of the neuronal response in the non-cooperative single-compartment model was analyzed. 


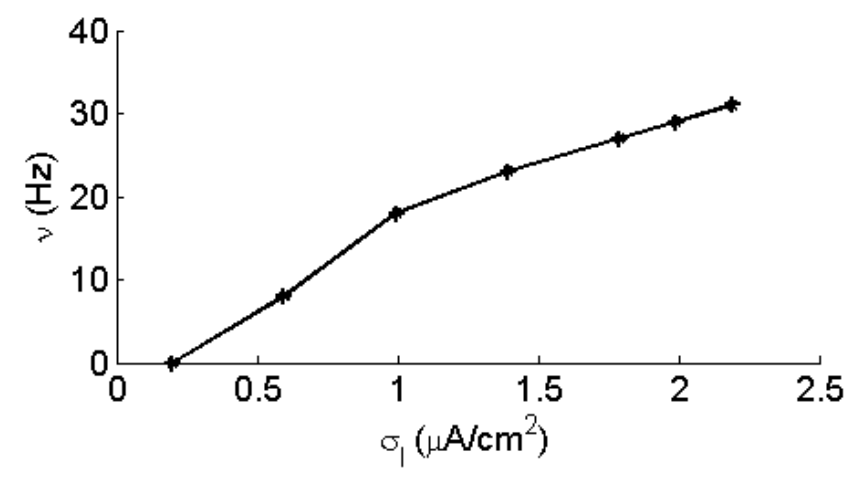

Figure 8.8: Effect of $\sigma_{I}$ on model firing rate. Noisy current with $\mu_{I}=0, \tau_{I}=5$ ms and with varying $\sigma_{I}$ was injected into the model. The firing rate increased with increasing $\sigma_{I}$ as continuously, as for $\mu_{I}$.

\subsubsection{Firing Rate}

The firing rate of the model with classical gating kinetics was almost linearly dependent on $\sigma_{I}$ (Figure 8.8). As it will be seen in the following sections, the model represents a type-I neuron (Figure 3.17), therefore, the continuous and gradual increase of $\nu$ with increasing $\sigma_{I}$ was as predicted. On the other hand, the effect of $\tau_{I}$ was not that obvious as in Figure 8.9. The slight increase in $\nu$ only occurred under high $\sigma_{I}\left(2 \mu \mathrm{A} / \mathrm{cm}^{2}\right)$. Therefore, it could be concluded that $\sigma_{I}$ has a greater impact on the firing rate of the model compared to $\tau_{I}$ and the relationship between $\nu$ and $\sigma_{I}$ is almost linear.

\subsubsection{Maximum Amplitude of Action Potentials}

As for the firing rate, the maximum value of the action potentials were almost linearly dependent on $\sigma_{I}$ (Figure 8.10). Although a slight decrease was observed for increasing $\tau_{I}$ under high $\sigma_{I}\left(2 \mu \mathrm{A} / \mathrm{cm}^{2}\right)$, the change was less than $10 \mathrm{mV}$ (Figure 8.11). This decrease might be due to the fact that there will be faster oscillations in the membrane potential by small $\tau_{I}$, which might decrease the time between two spikes and hence, the peak amplitude of action potentials. However,for $\sigma_{I}=1 \mu \mathrm{A} / \mathrm{cm}^{2}$, the peak values remained constant by increasing $\tau_{I}$. This might be due to the fact that the changes were too small to detect. In both cases, the values were very close.

\subsubsection{Subthreshold Membrane Potential Oscillations}

The subthreshold oscillations in the model were analyzed using standard deviation of the membrane potential, $\sigma_{V} \cdot \sigma_{V}$ was positively dependent on $\sigma_{I}$ and the relationship 


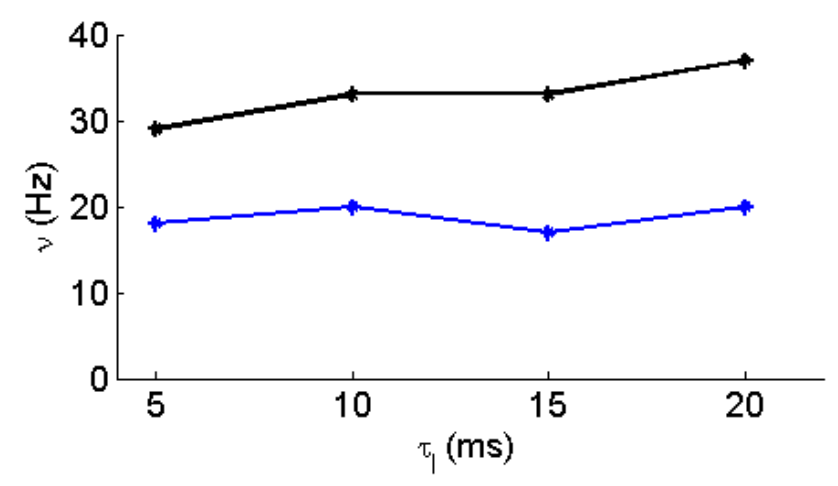

Figure 8.9: Effect of $\tau_{I}$ on model firing rate. Noisy current with $\mu_{I}=0, \sigma_{I}=1$ $\mu \mathrm{A} / \mathrm{cm}^{2}$ (blue) and $2 \mu \mathrm{A} / \mathrm{cm}^{2}$ (black) and with varying $\tau_{I}$ was injected into the model. The firing rate increased increased slightly with increasing $\tau_{I}$ for $\sigma_{I}=2 \mu \mathrm{A} / \mathrm{cm}^{2}$ and was independent of $\tau_{I}$ for $\sigma_{I}=1 \mu \mathrm{A} / \mathrm{cm}^{2}$.

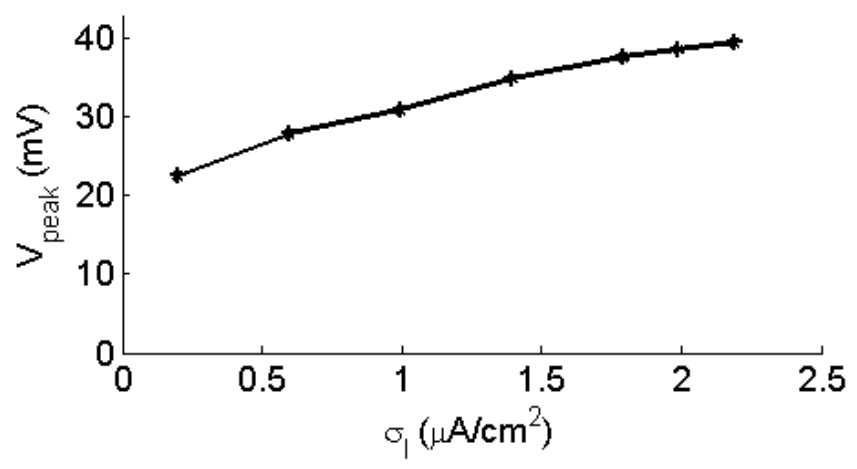

Figure 8.10: Effect of $\sigma_{I}$ on action potential peak values. Noisy current with $\mu_{I}=0, \tau_{I}=5 \mathrm{~ms}$ and with varying $\sigma_{I}$ was injected into the model. 
118 Chapter 8: Single-Compartment Conductance-Based Cooperative Channel Gating (SC-COOP) Model

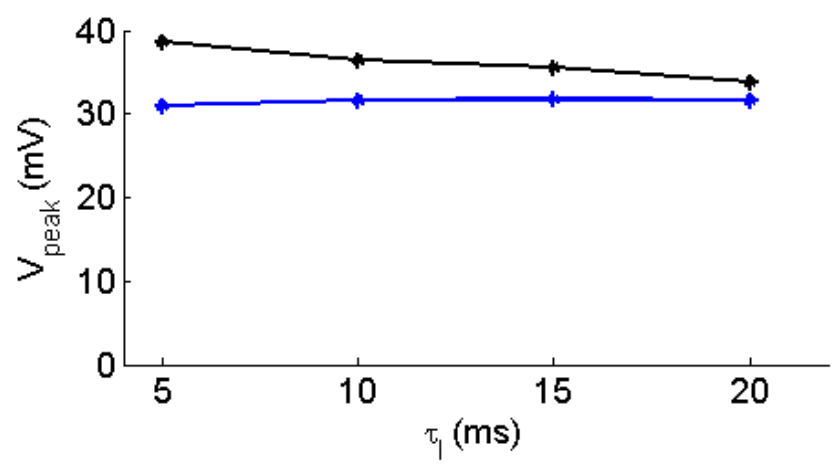

Figure 8.11: Effect of $\tau_{I}$ on model action potential peak values. Noisy current with $\mu_{I}=0, \sigma_{I}=1 \mu \mathrm{A} / \mathrm{cm}^{2}$ (blue) and $2 \mu \mathrm{A} / \mathrm{cm}^{2}$ (black) and with varying $\tau_{I}$ was injected into the model.

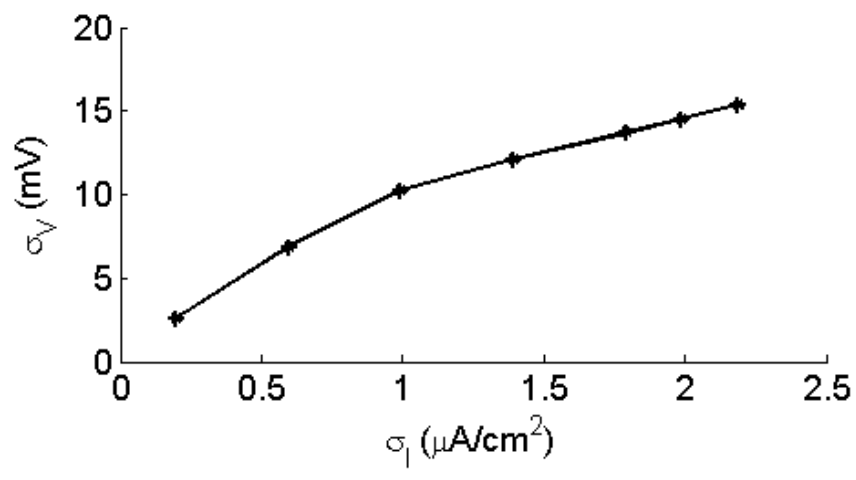

Figure 8.12: Effect of $\sigma_{I}$ on subthreshold membrane potential deviations. Noisy current with $\mu_{I}=0, \tau_{I}=5 \mathrm{~ms}$ and with varying $\sigma_{I}$ was injected into the model.

was almost exponential(Figure 8.12). On the other hand, the effect of $\tau_{I}$ was not as obvious as $\sigma_{I}$. A slight positive dependency on $\tau_{I}$ was observed with high values of $\sigma_{I}$, however, for smaller $\sigma_{I}, \sigma_{V}$ was independent of $\tau_{I}$ (Figure 8.13). 


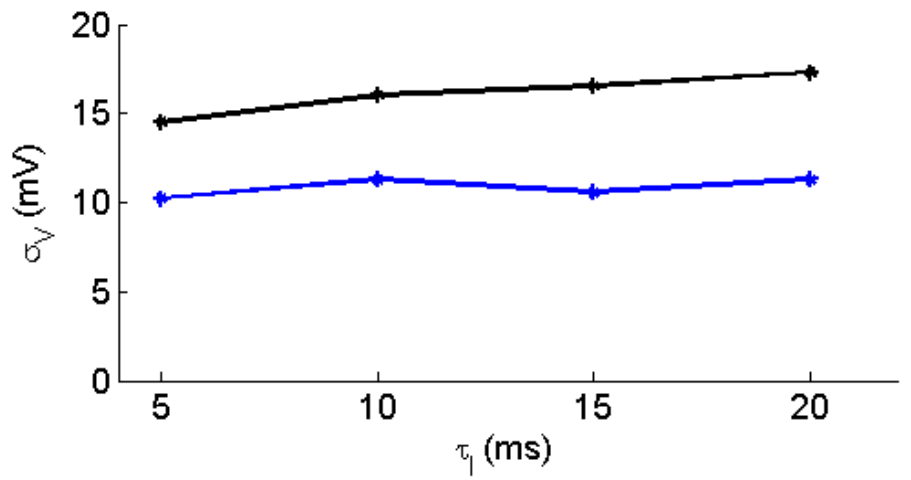

Figure 8.13: Effect of $\tau_{I}$ on subthreshold membrane potential deviations. Noisy current with $\mu_{I}=0, \sigma_{I}=1 \mu \mathrm{A} / \mathrm{cm}^{2}$ (blue) and $2 \mu \mathrm{A} / \mathrm{cm}^{2}$ (black) and with varying $\tau_{I}$ was injected into the model. 


\subsection{Impact on Frequency-Current ( $\nu$-I) Relation- ship}

Neurons are categorized as type-I or type-II according to the relationship between their firing rate and the electrode current. Type-I neurons exhibit a firing rate that rises continuously from zero as a function of the current. On the other hand, the firing rate of type-II neurons jumps discontinuously to a non-zero value around threshold potentials. The original WB model represents a type-I neuron, therefore, it was expected that our model is also a type-I neuron model. The results (Figures 8.14) support our predictions. The difference between the firing rates for current values higher than $2 \mu \mathrm{A} / \mathrm{cm}^{2}$ can be explained by the fact that SC-COOP model used a modified version of membrane currents given in the study and it does not include synaptic current contributions(Wang and Buzsàki [183]).

The frequency-current curves were highly sensitive to the activation of cooperativity (Figure 8.15). Increase in coupling strength and cooperativity percentage induce increase in the slope of $\nu$-I curves under constant current injection. The difference between the maximum and minimum slopes is on the range of $3.5 \mathrm{~Hz} . \mathrm{cm}^{2} / \mu \mathrm{A}$.

The frequency-current relationship in response to noisy current injection $\left(\sigma_{I}=\right.$ $0.08 \mu \mathrm{A} / \mathrm{cm}^{2}, \tau_{I}=5 \mathrm{~ms}$ ) also exhibits sensitivity to variations in the coupling strength and cooperativity percentage (Figure 8.17). Though slightly (maximum $2.5 \mathrm{~Hz} . \mathrm{cm}^{2} / \mu \mathrm{A}$ ), the slopes of the curves increase with increasing coupling strength. This is more pronounced when a larger fraction of the channel population is coupled (Figure 8.18). Compared to the constant current injection, the phase diagram pattern for noisy current injection exhibits smooth borders between increasing steps and a continuous and gradual increase for increasing coupling strength and cooperativity fraction after $p=10 \%$. 

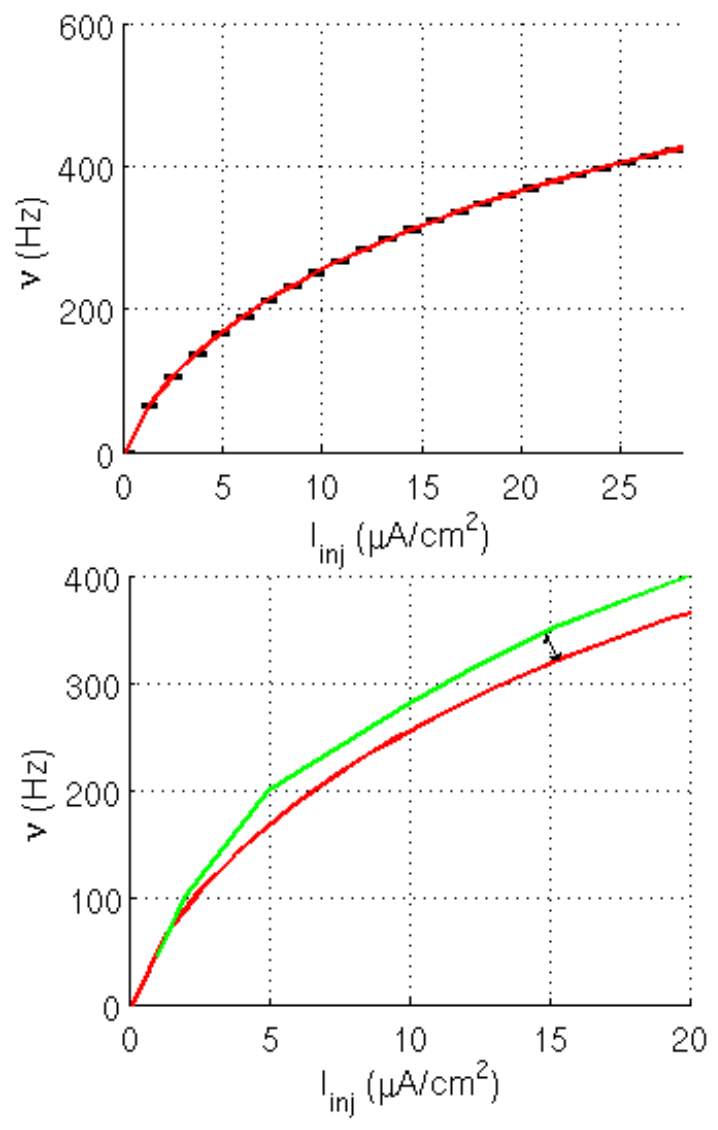

Figure 8.14: The comparison of $\nu$-I relationship in SC-COOP and original WB models. Upper figure: $\nu$-I curve for SC-COOP model. The black lines are the error bars for $\nu$ at the corresponding current values. The error under constant current injection is very close to 0 for all measured values. Lower figure: Comparison of SC-COOP and WB model (Wang and Buzsàki [183]). The red line represents the modified WB (non-cooperative SC-COOP) model. The green line represents the original WB model. The black arrow indicates the difference between the two curves. Both models are type-I neuron models, as expected. 


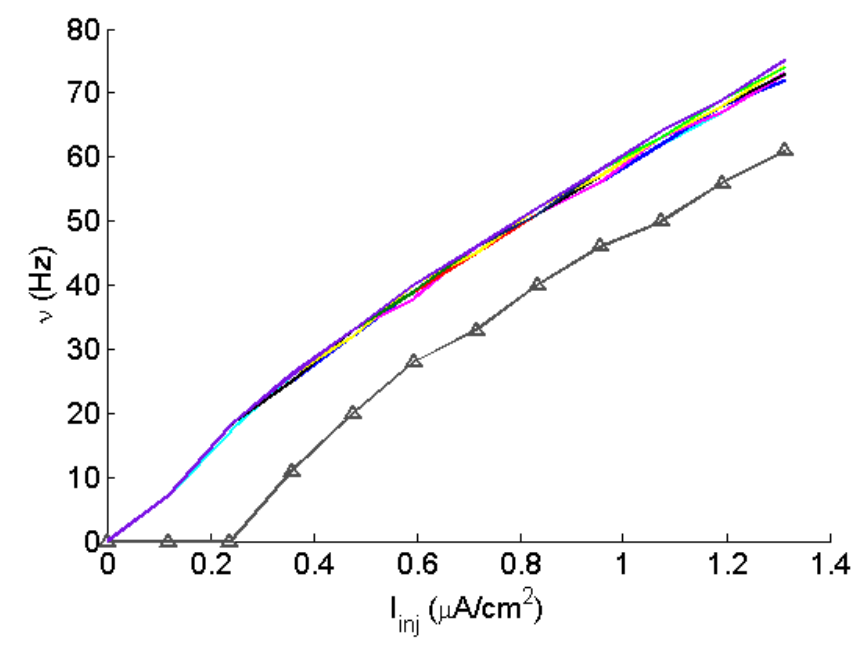

Figure 8.15: The $\nu$-I curves for $K J=800 \mathbf{~ m V}$ under constant current injection. Gray: statistically independent (classical) channel gating, $p=0$; cyan: $p$ $=5 \%$; blue: $p=10 \%$; magenta: $p=15 \%$; red: $p=20 \%$; black: $p=25 \%$; yellow: $p=30 \%$; dark blue: $p=35 \%$; dark green: $p=40 \%$; light green: $p=45 \%$; purple: $p$ $=50 \%$. Some curves may not be visible due to overlapping. The error bars are not indicated as the error is negligible in all cases.

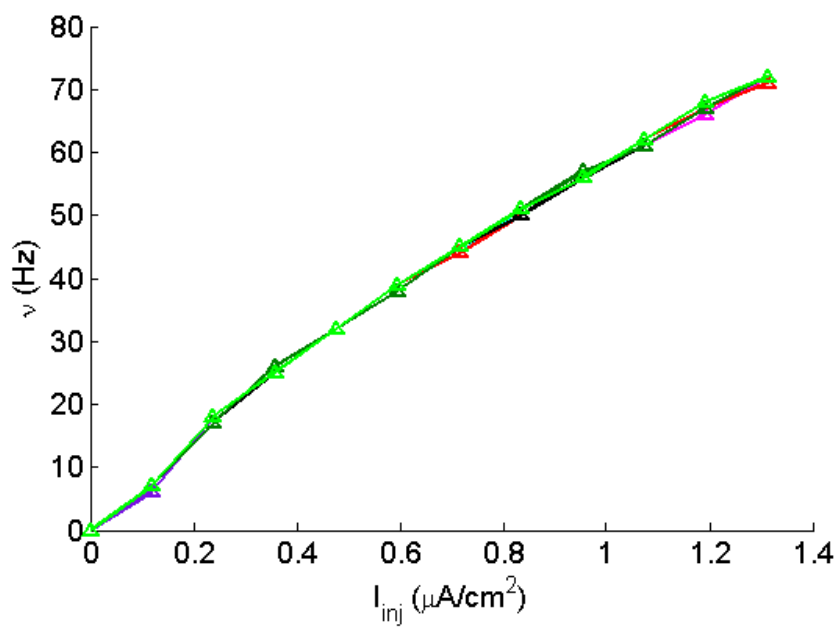

Figure 8.16: The $\nu$-I curves for $p=\mathbf{1 0} \%$ under constant current injection. purple: $K J=20 \mathrm{mV}$; cyan: $K J=100 \mathrm{mV}$; blue: $K J=200 \mathrm{mV}$; magenta: $K J=$ $300 \mathrm{mV}$; red: $K J=400 \mathrm{mV}$; black: $K J=500 \mathrm{mV}$; dark green: $K J=600 \mathrm{mV}$; light green: $K J=800 \mathrm{mV}$. Some curves may not be visible due to overlapping. The error bars are not indicated as the error is negligible in all cases. 


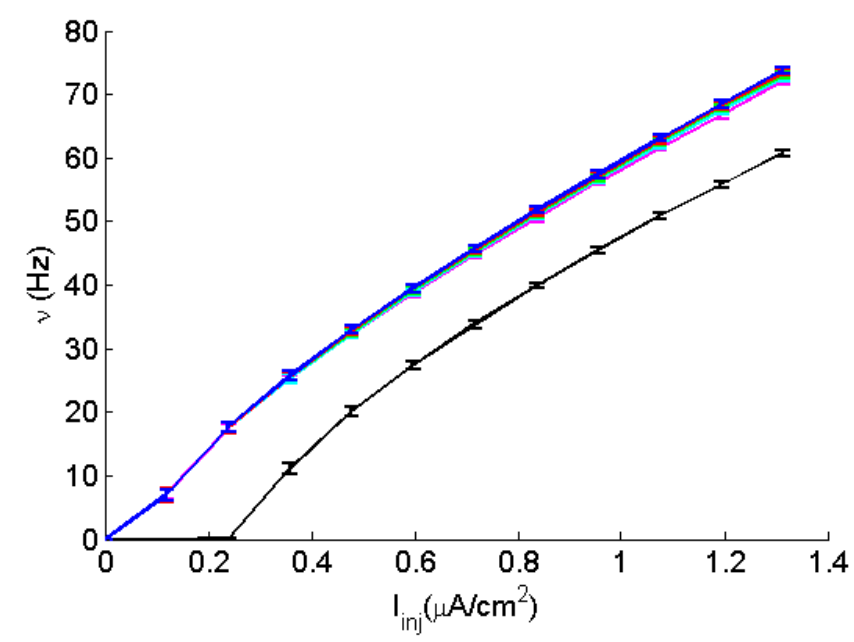

Figure 8.17: The $\nu$-I curves for $K J=600 \mathbf{m V}$ under noisy current injection. Black: statistically independent (classical) channel gating, $p=0$; magenta: $p=10 \%$; cyan: $p=20 \%$; light green: $p=30 \%$; red: $p=40 \%$; blue: $p=50 \%$. Some curves may not be visible due to overlapping. The error bars for the frequencies at the corresponding current values are estimated as $2^{*} \sigma_{f}$.

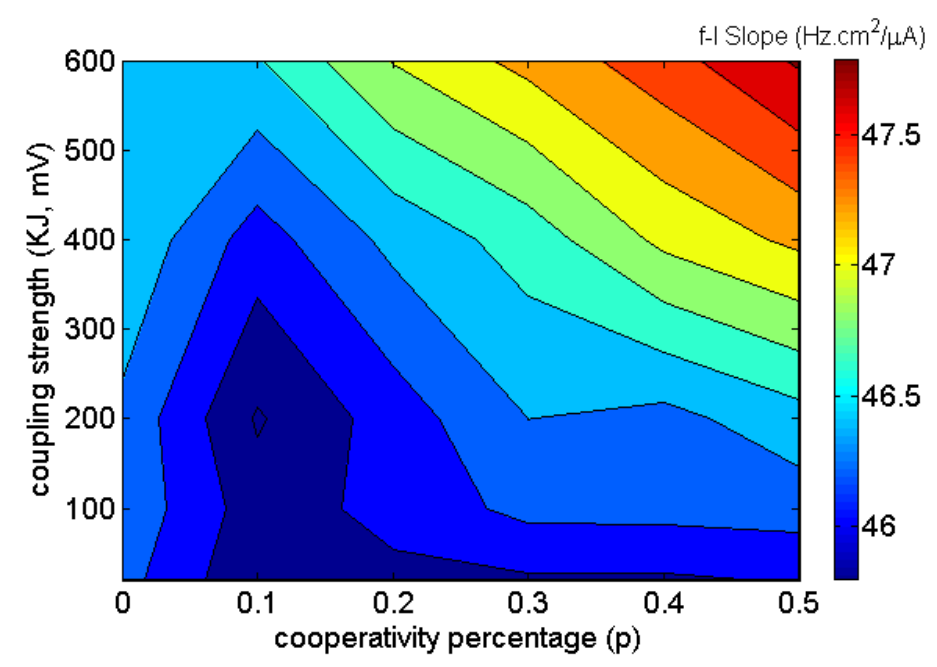

Figure 8.18: The phase diagram for $\nu$-I curves under noisy current injection $\left(\sigma_{I}=0.08 \mu \mathbf{A} / \mathbf{c m}^{2}, \tau_{I}=\mathbf{5} \mathbf{m s}\right)$. The diagram shows the increase in the slope of $\nu$-I curves with increasing coupling strength and cooperativity percentage. The slope is estimated for the part of the curves that correspond to $0.6-1.3 \mu \mathrm{A} / \mathrm{cm}^{2}$ interval. 
124 Chapter 8: Single-Compartment Conductance-Based Cooperative Channel Gating (SC-COOP) Model

\subsection{Summary and Discussion}

Single-compartment cooperative (SC-COOP) model was first developed by Min Huang in MATLAB. Here, I implemented the model in $\mathrm{C}++$ to test the implementation of cooperative gating kinetics in a classical conductance-based model, which has the same code structure with more complicated and more realistic models (see Chapter 9 ), but which is much simpler as it lacks the complicated geometry. I also used the previous results as control groups.

The results described in this chapter and by Huang et al. [89] provided us insight about the effect of inter-channel coupling on single channel activation and inactivation (Figure 8.3). For higher values than critical coupling strength, the critical potential threshold shifted towards more negative values and the activation curve for $\mathrm{Na}_{V}$ took the form of a step function instead of a Boltzmanian, which means that the activation became simply an all-or-none event. Same type of behavior (a negative shift in threshold potential for activation and an abrupt opening) was also reported by Undrovinas et al. [179]. To sum up, the effective coupling strength is a significant factor for the determination of critical activation threshold and of the duration that a channel remains open, due to the changes in the opening and closing rates (Undrovinas et al. [179]).

Additionally, the cooperative activity affects the AP waveform, especially at the onset. The onset rapidness strongly depends on the value of coupling strength, adversely, the cooperativity percentage does not play a significant role as long as $p<$ $30 \%$ and $K J<400 \mathrm{mV}$ (Figure 8.7). Furthermore, the biphasic action potentials as observed frequently in cortical neurons (Eccles et al. [53]; Naundorf et al. [130],[132]; Bean [15]) were also observed for $p<35 \%$ and $K J>400 \mathrm{mV}$.

Considering the relationship between onset rapidness and cut-off frequency (FourcaudTrocme et al. [63]), the rapid AP onsets due to cooperative activity of $\mathrm{Na}^{+}$channels should imply high cut-off frequencies that were already reported for cortical neurons (Köndgen et al. [100]; Boucsein et al. [20]). The proceeding step of this research, therefore, should be the investigation of this relationship.

In conclusion, SC-COOP model serves well to the purpose of testing the implementation of cooperative gating kinetics and its effects. The results obtained from the model suggest the possibility of having cooperative activity of $\mathrm{Na}^{+}$channels instead of stochastically independent activity in neuronal AP generating mechanisms. To test the hypothesis further, one needs the utilization of a more realistic model of AP initiation. Therefore, next, I used a multi-compartmental conductance-based model which includes the axon initial segment (AIS) as the initiation site of the ac- 
tion potentials. As AIS should be the host to AP generating mechanisms, cooperative channel gating was implemented only in this region. Also, the debates about the $\mathrm{Na}^{+}$ channel density distribution was considered and the possible contribution with or without cooperativity was also investigated. 
126 Chapter 8: Single-Compartment Conductance-Based Cooperative Channel Gating (SC-COOP) Model 


\section{9 Multi-compartmental Cooperative Axon Initial Segment (MCCAIS) Model}

The cooperative model was first implemented as a spherical single compartment model as described in the previous chapter. However, neuron models with more complex, multi-compartmental geometry would be needed for a more precise approach to the actual effects of cooperative channel gating on AP generation of a cortical neuron in its physiological environment, since the spatial heterogeneity of electrical properties might significantly affect the dynamics of AP generation and propagation. In addition to the geometry and heterogeneous electrical properties, the heterogeneous distribution of channel densities (i.e. for $\mathrm{Na}_{V}$ channels) along the neuronal membrane is particularly important. The recent studies pointed out an increased $\mathrm{Na}_{V}$ channel density at the proximity of the site of AP initiation (see Section 2.2), which is AIS in the myelinated neurons. But:

- Which parameters determine the site of AP initiation?

- What is the most realistic range of $\mathrm{Na}_{V}$ channel density at the site of initiation?

- Could a model with cooperative activity of sodium channels reproduce the AP shape and onset rapidness as observed in the cortical neurons?

In this chapter, we address these critical questions and investigate their answers on a multi-compartmental conductance-based model. The model consists of

- a thin cable-like extension with homogeneous thickness and homogeneous electrical properties to represent the dendritic assembly,

- an octagon-shaped ball with homogeneous electrical properties to represent soma,

- a thin cable-like extension with heterogeneous thickness and heterogeneous electrical properties to represent the axon,

- a small octagon-shaped bleb to end the axon. 
The myelinated axon itself can be divided into further subgroups as the axon hillock (AH), the axon initial site (AIS), the myelinated compartments and the nodes of Ranvier (NR).

First multi-compartmental model that included AIS as a separate part with realistic features was constructed by Mainen et al. [117]. Since then, many of the multi-compartmental models included this segment, i.e. to investigate the AP initiation dynamics. However, there is no model up to now (to our knowledge) that incorporated cooperative channel gating kinetics in AIS. The fact that there is still no experimental evidence about the cooperative activity of $\mathrm{Na}^{+}$channels in neurons may be the underlying reason. Obviously, this type of experimental design is a difficult one. However, the existence of $\mathrm{Na}^{+}$channel coupling in cardiomyocytes (Undrovinas et al. [179]) and the very fast onset dynamics observed in cortical neurons (Naundorf et al. [132]) may imply the possible existence of such activity in AIS.

When similar ion gating properties are assumed, a greater density of sodium channels in AIS should lead to a significantly larger $\mathrm{Na}^{+}$current in this site. This expectation is challenged with the electrophysiological studies with the cell-attached and outside-out patch clamp mode, which revealed that there is only a small difference in $\mathrm{Na}^{+}$current between soma, proximal dendrites and AIS (Colbert and Pan [39]; Kole et al. [97]). These findings are incompatible with what should be expected from large density differences.

\subsection{Model Methods}

\subsubsection{Numerical Methods}

The model was implemented using $\mathrm{C}++$ in Linux environment. The implicit backward Euler integration method was used with a $10 \mu$ s time step. To maintain a constant $\nu_{0}$ between trials for specific experimental designs, a current adapter was also included in the code such that it changes $\mu_{I}$ of the injected current (see section 9.1.5) until the desired $\nu_{0}$ is obtained.

\subsubsection{Model Geometry}

The morphology of the MCCAIS neuron model was based on the experimental data from pyramidal neurons (data kindly provided by Michael J. Gutnick, Koret School of Veterinary Medicine, The Hebrew University of Jerusalem, Israel). A similar model with only canonical gating kinetics was also used to study the AP onset and propagation by Huang [88] and the findings from this study provided a second control group for our simulations (data not submitted). 


\begin{tabular}{|c||c|c|c|c|c|}
\hline PART & $\mathbf{N}_{\text {comp }}$ & $l_{\text {comp }}(\mu \mathbf{m})$ & $a_{\text {in }}^{*}(\mu \mathbf{m})$ & $a_{\text {out }}^{*}(\mu \mathbf{m})$ & $C_{m}\left(\mathbf{n F} / \mu \mathbf{m}^{2}\right)$ \\
\hline \hline Dendrite 1 & 6 & 50 & 0.5 & 0.5 & $10^{-5}$ \\
\hline Dendrite 2 & 10 & 10 & 0.5 & 1 & $10^{-5}$ \\
\hline Soma & 20 & 2 & 1 & 1.5 & $10^{-5}$ \\
\hline Axon Hillock & $n$ & 2 & 1.5 & $1.5-a_{x}$ & $10^{-5}$ \\
\hline Axon Initial Segment & $25-n$ & 2 & $1.5-a_{x}$ & 0.5 & $10^{-5}$ \\
\hline Myelinated Segment & 5 & 10 & 0.5 & 0.5 & $2^{*} 10^{-6}$ \\
\hline Node of Ranvier & 1 & 2 & 0.5 & 0.5 & $10^{-5}$ \\
\hline Bleb & 5 & 2 & 0.5 & 0.5 & $10^{-5}$ \\
\hline
\end{tabular}

Table 9.1: The parameters of Multi-Compartmental Cooperative AIS (MCCAIS) Model. Please also see Figure 9.1 and text.

The details of the model are given in Figure 9.1 and Table 9.2.

\subsubsection{Passive Electrical Parameters}

The membrane capacitance $\left(\mathrm{C}_{m}\right)$ was $0.1 \mathrm{nF} / \mathrm{mm}^{2}$. The membrane resistance $\left(\mathrm{r}_{m}\right)$ was $1 \mathrm{M} \Omega . \mathrm{mm}^{2}$, longitudinal membrane resistance, $r_{l}$, was either 3 or $5 \mathrm{M} \Omega . \mathrm{mm}$ and the membrane time constant was estimated as $10 \mathrm{~ms}$. A summary of the passive parameters for each part is given in Table 9.2.

\subsubsection{Ionic Channel Density Distribution and Gating Kinet- ics}

As in single-compartment cooperative (SC-COOP) model, MCCAIS model was also built by modifying the conductance-based Wang-Buzsaki (WB) model. The details of the model and, the classical and cooperative channel gating kinetics were given in Chapter II. Furthermore, cooperative channel gating was implemented also for MCCAIS model, and as the name implies, only the $\mathrm{Na}_{v}$ channels of AIS were assumed to act cooperatively. Therefore, the cooperativity parameters (cooperativity percentage $p$ and coupling strength $K J$ ) were set to zero in the remaining compartments.

The different views on the sodium channel density of AIS was discussed in Part I in detail. Considering the broad variety of experimental findings and model assumptions, the sodium channel conductance $g_{N a}$ in the different parts of MCCAIS model varied from $300 \mathrm{pS} / \mu \mathrm{m}^{2}$ to $3000 \mathrm{pS} / \mu \mathrm{m}^{2}$. Somatic $g_{N a}, g_{\text {soma }}$, was equal to $300 \mathrm{pS} / \mu \mathrm{m}^{2}$ and remained constant. The conductance in the the dendrites $\left(g_{\text {dend }}\right)$, axon hillock $\left(g_{A H}\right)$, myelinated parts $\left(g_{m y e}\right)$ and terminal bleb $\left(g_{b l e b}\right)$ also remained constant and 
130 Chapter 9: Multi-compartmental Cooperative Axon Initial Segment (MCCAIS) Model

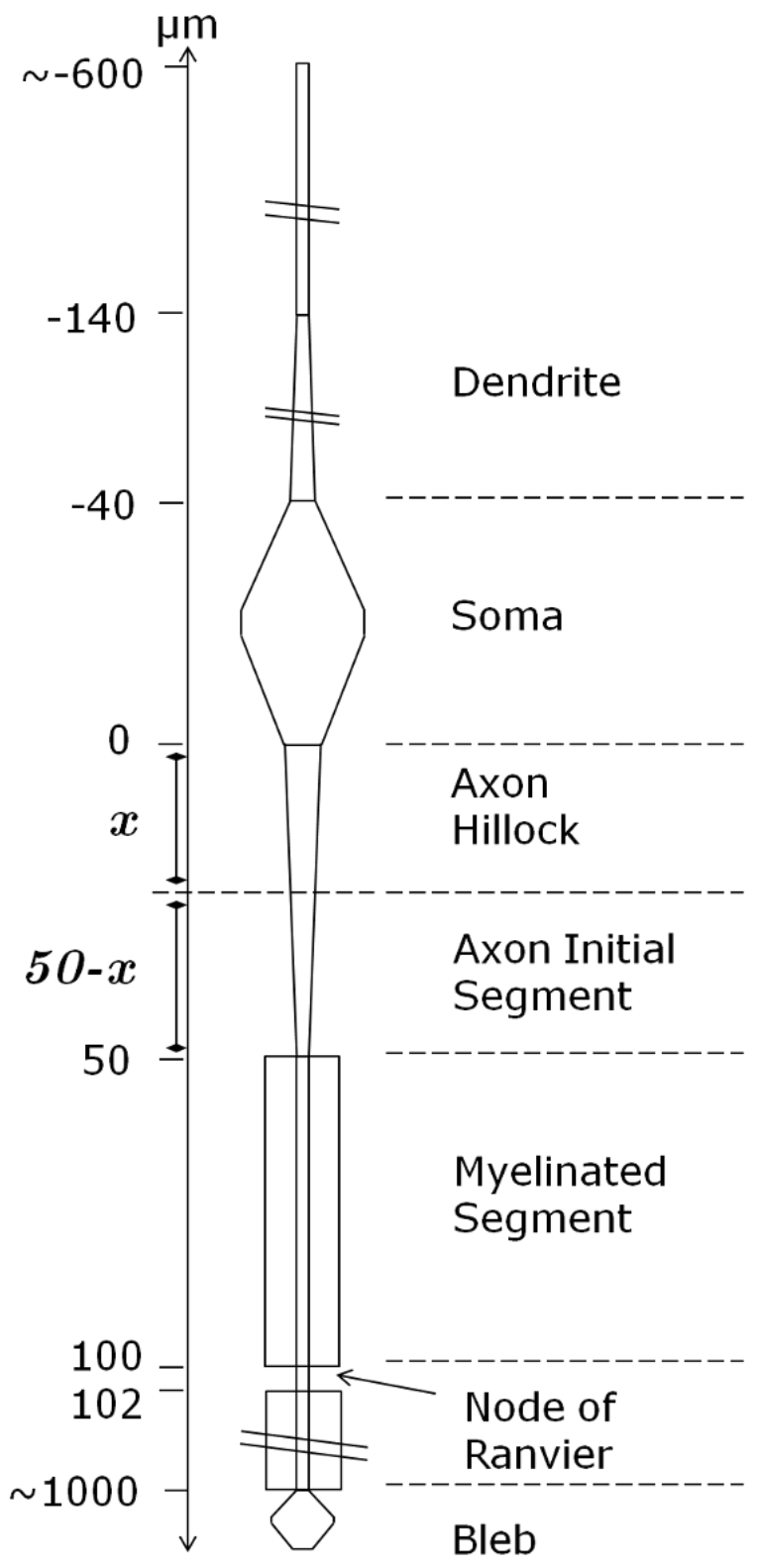

Figure 9.1: The geometry of Multi-Compartmental Cooperative AIS (MCCAIS) model. 


\begin{tabular}{|c||c|c|c|c|}
\hline PART & $\begin{array}{c}\mathbf{g}_{N a} \\
\left(\mathbf{p S} / \mu \mathbf{m}^{2}\right)\end{array}$ & $\begin{array}{c}\mathbf{g}_{N a} \\
\left(\mathbf{g}_{\text {soma }}\right)\end{array}$ & $\begin{array}{c}\mathbf{g}_{K} \\
\left(\mathbf{p S} / \mu \mathbf{m}^{2}\right)\end{array}$ & $\begin{array}{c}\mathbf{g}_{\text {Leak }} \\
\left(\mathbf{p S} / \mu \mathbf{m}^{2}\right)\end{array}$ \\
\hline \hline Dendrite & 100,300 & $0.3,1$ & 150 & 1 \\
\hline Soma & 300 & 1 & 150 & 1 \\
\hline Axon Hillock & 300,3000 & 1,10 & 150 & 1 \\
\hline Axon Initial Segment & $300-3000$ & $1-10$ & 150 & 1 \\
\hline Myelinated Segment & 0 & 0 & 150 & 0.2 \\
\hline Node of Ranvier & $300-3000$ & $1-10$ & 150 & 1 \\
\hline Bleb & 300 & 1 & 150 & 1 \\
\hline
\end{tabular}

Table 9.2: The channel density distribution for MCCAIS. A dash between two values means that the value of parameter was chosen in this interval. A comma between two values means that the parameter took either the first or the second value.

were set as shown in Table 9.2. The conductances in the other parts, namely in AIS $\left(g_{A I S}\right)$ and nodes of Ranvier $\left(g_{N R}\right)$ were varied from 1-10-fold $g_{s o m a}$.

\subsubsection{Current Injection and Data Analysis}

The design of the current injection was the same as used for SC-COOP model.

Data were obtained from the simulation as either potential traces, spike times or both. Data analysis were performed in MATLAB v.7.0.0 and v7.2 .0 using customwritten functions and procedures.

$\dot{V}\left(t_{i}\right)=10 \mathrm{~V} / \mathrm{s}$ is chosen to define the threshold as in the previous models (Part II, Chapter II and Part III, Chapter II). Then, the slope of the phase plot ( $V$ vs $\dot{V})$ at $\dot{V}\left(t_{i}\right)$ is measured to obtain the onset rapidness, $\Gamma$.

\subsection{Action Potential Waveform in MCCAIS Model}

The single-compartment cooperative (SC-COOP) model was used previously to characterize the effect of cooperative in a simpler model, and the results indicated that the cooperativity reproduced the sharp AP onsets that Hodgkin-Huxley model failed to reproduce. Therefore, a similar behavior was expected for MCCAIS model.

The results from MCCAIS model also confirmed that, APs with high $\Gamma$ (as in cortical neurons (Naundorf et al. [132])) can be generated when cooperative $\mathrm{Na}^{+}$channel gating is implemented in a classical Hodgkin-Huxley type model (Figure 9.2). This 

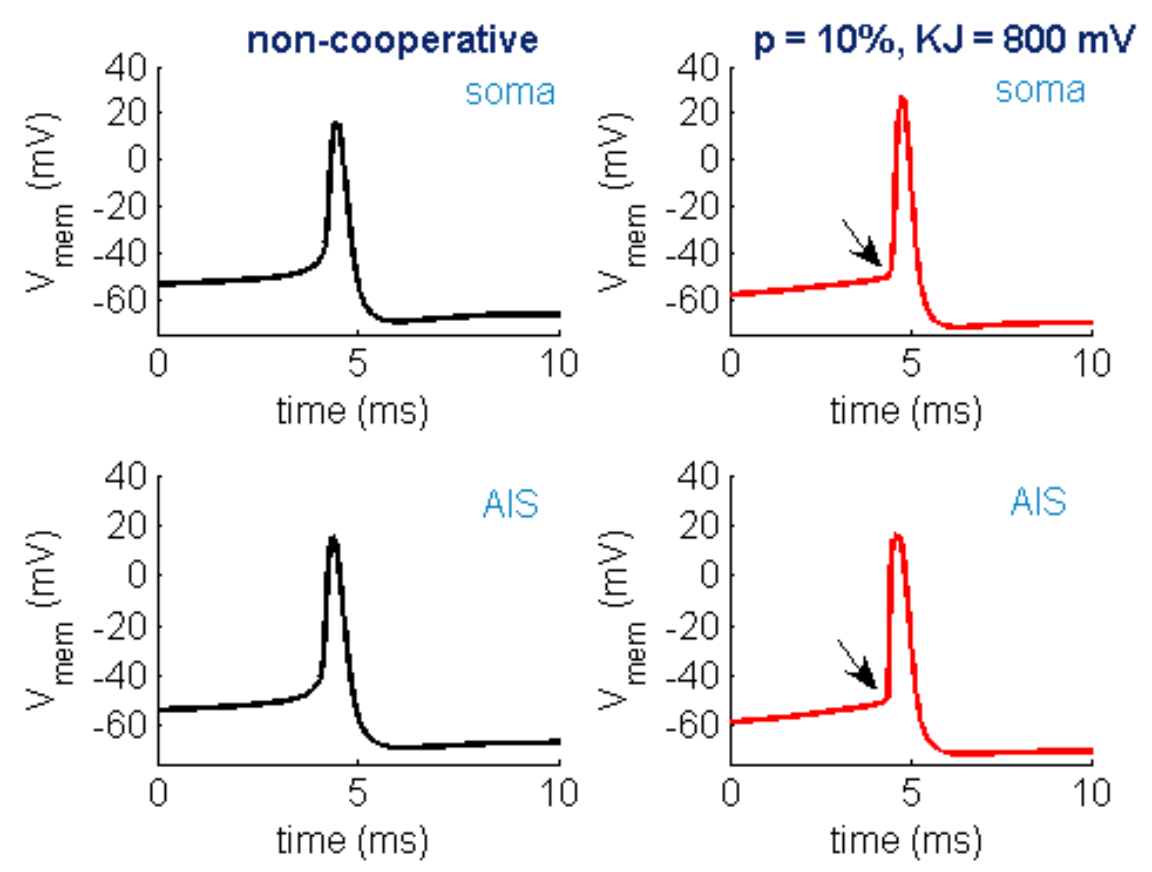

Figure 9.2: AP waveforms in MCCAIS Model $\left(g_{\text {soma }}=g_{\text {dend }}\right)$. The APs were obtained from the simulations on non-cooperative and cooperative MCCAIS models. The cooperativity parameters are as given in the figure. $g_{A I S}=g_{N R}=3^{*} g_{\text {soma }}=$ $3^{*} g_{\text {dend }}$. A noisy current with $\tau_{I}=5 \mathrm{~ms}$ and an automatically adjusted $\mu_{I}$ was injected to maintain $\nu_{0}=10 \mathrm{~Hz}$. $\sigma_{I}$ was set to give $\sigma_{V} \cong 5 \mathrm{mVThe}$ arrows indicate the "kink"s.

behavior was evident without the need of unrealistically high $\mathrm{Na}^{+}$channel density at AIS (Mainen et al. [117]). For instance, the "kink" in Figure 9.2 was obtained at $g_{A I S}$ $=3^{*} g_{\text {soma }}$ with a strong $(K J=1000 \mathrm{mV})$ coupling of a small fraction $(p=10 \%)$ of channels. The "kink"s were also evident in the phase plots of non-cooperative and cooperative models (Figure 9.3).

The simulations were performed for both $g_{\text {soma }}=g_{\text {dend }}$ and $g_{\text {soma }}=3^{*} g_{\text {dend }}$. In both cases, the AP waveforms and phase plots were similar (Figure 9.4 and 9.5) and cooperative models reproduced the "kink"s that were observed for the onset of cortical APs (Naundorf et al. [132]).

\subsection{Initiation Site in MCCAIS Model}

The site of action potential initiation was always topic of interest since 1950s. The experimental findings up to date supported the view that AIS is the initiation site in 


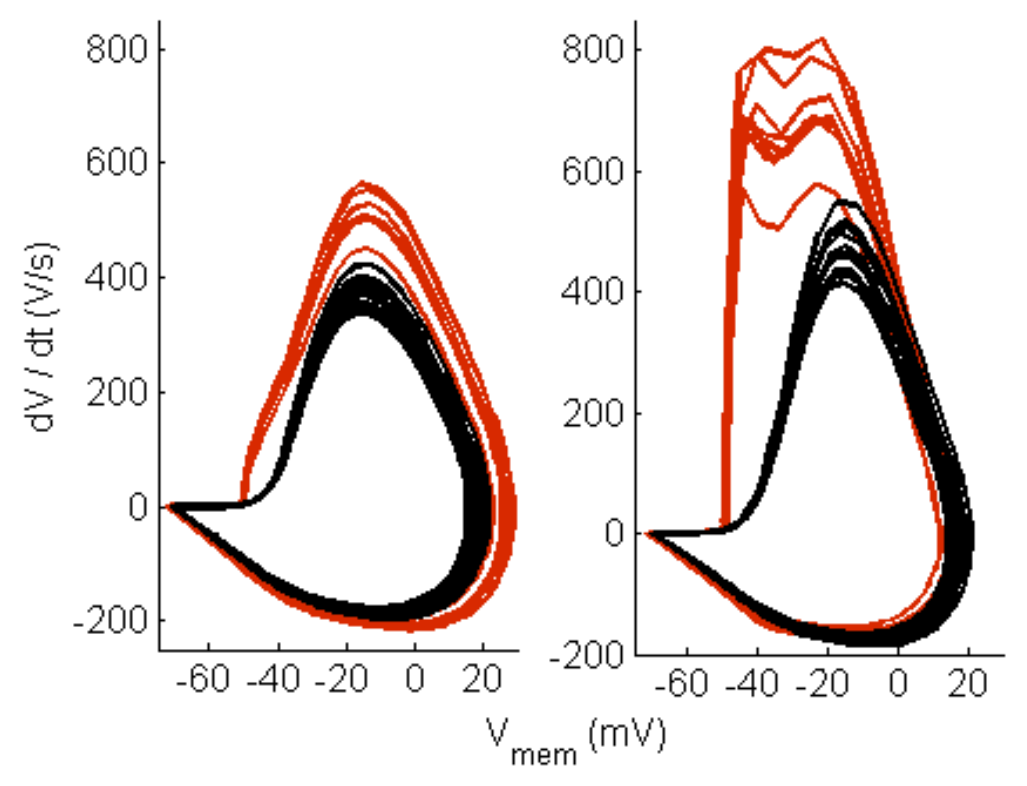

Figure 9.3: Phase Plot Diagrams $\left(g_{\text {soma }}=g_{\text {dend }}\right)$. The phase plot diagrams of the APs given in Figure 9.2 are used for quantitative and quantitative (see section 9.5) comparison of non-cooperative and cooperative model AP onsets. Left: Phase plots for soma. Right:Phase plots for AIS. Black traces indicate the non-cooperative model, red traces indicate the cooperative model $(p=10 \%, K J=800 \mathrm{mV}) . g_{A I S}$ $=g_{N R}=3^{*} g_{\text {soma }}=3^{*} g_{\text {dend }}$. A noisy current with $\tau_{I}=5 \mathrm{~ms}$ and an automatically adjusted $\mu_{I}$ was injected to maintain $\nu_{0}=10 \mathrm{~Hz}$. $\sigma_{I}$ was set to give $\sigma_{V} \cong 5 \mathrm{mV}$ 

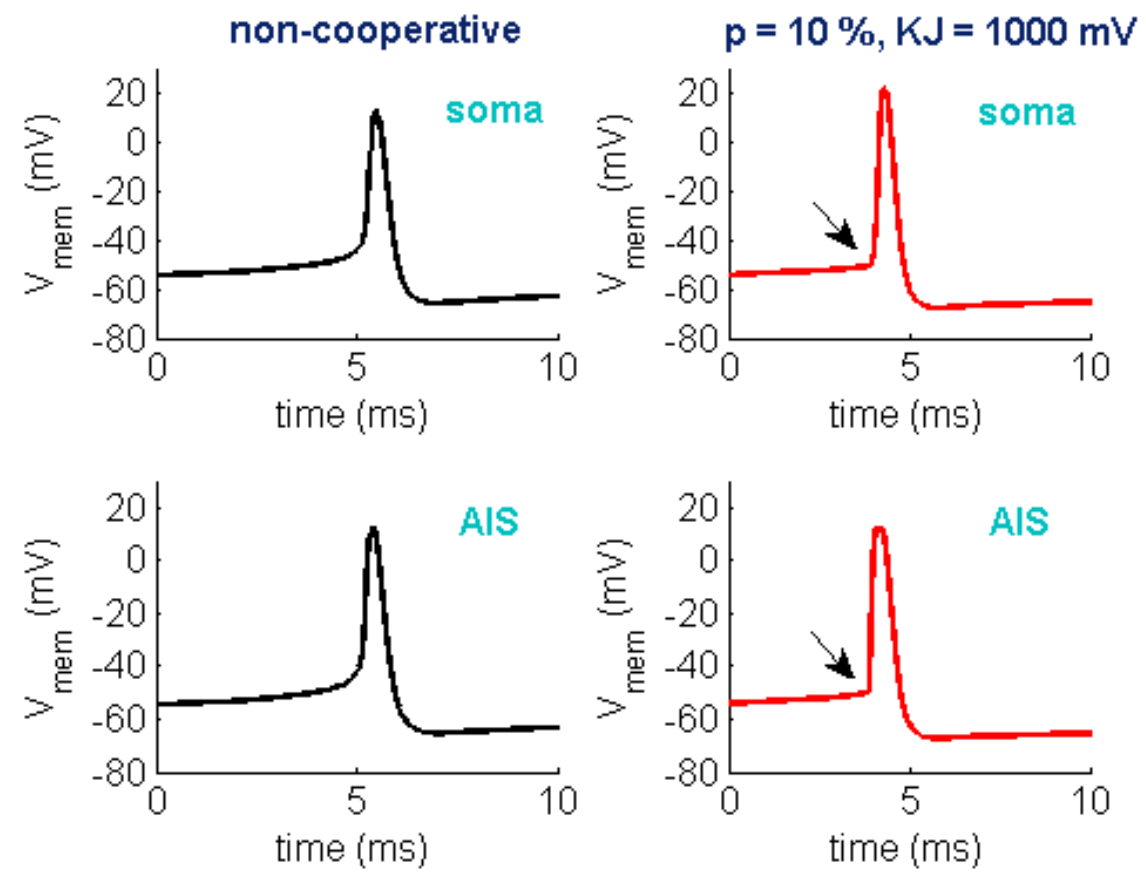

Figure 9.4: AP waveforms in MCCAIS Model $\left(g_{\text {soma }}=\mathbf{3}^{*} g_{\text {dend }}\right)$. The APs were obtained from the simulations on non-cooperative and cooperative MCCAIS models. The cooperativity parameters are as given in the figure. $g_{A I S}=g_{N R}=$ $3^{*} g_{\text {soma }}=9^{*} g_{\text {dend }}$. A noisy current with $\tau_{I}=5 \mathrm{~ms}$ and an automatically adjusted $\mu_{I}$ was injected to maintain $\nu_{0}=10 \mathrm{~Hz}$. $\sigma_{I}$ was set to give $\sigma_{V} \cong 5 \mathrm{mV}$ The arrows indicate the "kink"s. 

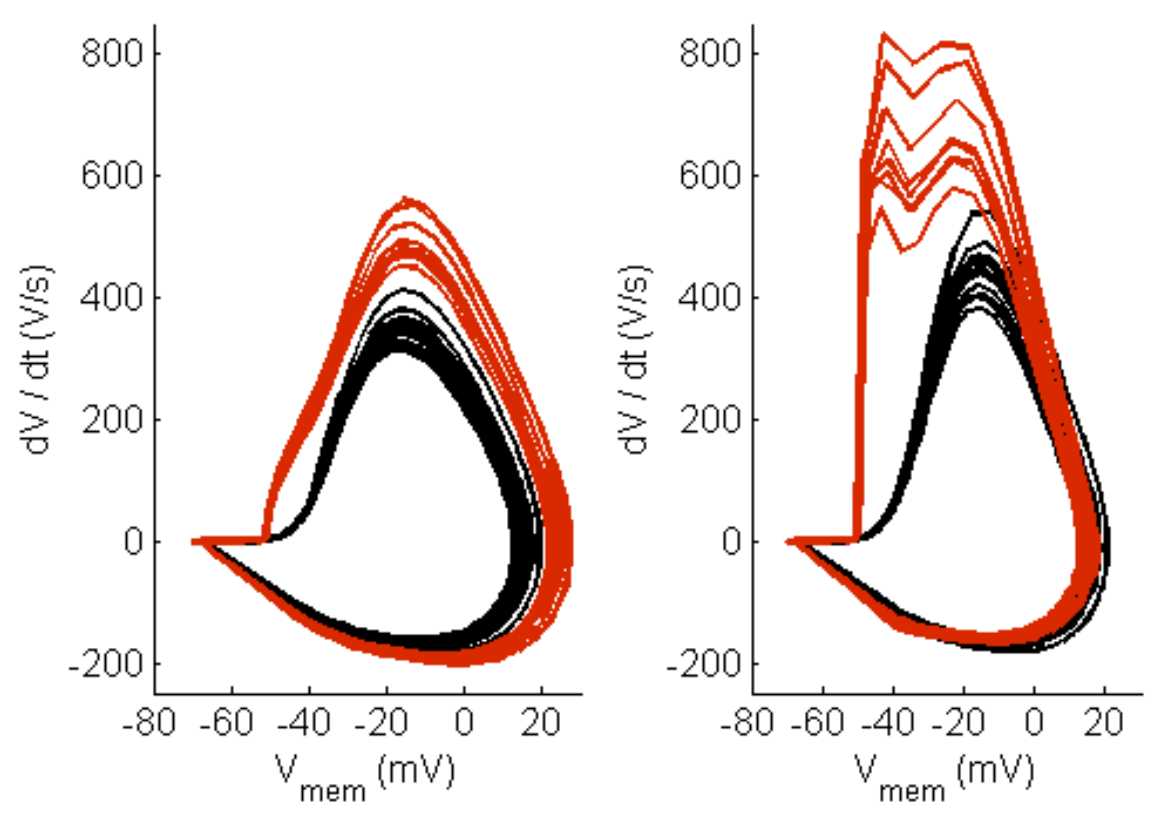

Figure 9.5: Phase Plot Diagrams $\left(g_{\text {soma }}=\mathbf{3}^{*} g_{\text {dend }}\right)$. The phase plot diagrams of the APs given in Figure 9.4 are used for quantitative and quantitative (see section 9.5) comparison of non-cooperative and cooperative model AP onsets. Left: Phase plots for soma. Right:Phase plots for AIS. Black traces indicate the non-cooperative model, red traces indicate the cooperative model $(p=10 \%, K J=1000 \mathrm{mV}) . g_{A I S}$ $=g_{N R}=3^{*} g_{\text {soma }}=9^{*} g_{\text {dend }}$. A noisy current with $\tau_{I}=5 \mathrm{~ms}$ and an automatically adjusted $\mu_{I}$ was injected to maintain $\nu_{0}=10 \mathrm{~Hz}$. $\sigma_{I}$ was set to give $\sigma_{V} \cong 5 \mathrm{mV}$ 
most of the myelinated neurons of the central nervous system (see section 2.2). The experimental and theoretical studies emphasized the importance of ionic, i.e. $\mathrm{Na}^{+}$, channel density distribution to locate the action potential initiation in a neuron (see section 2.2 for more detail). Therefore, parameters, such as $\mathrm{Na}^{+}$channel density, longitudinal resistance, $r_{l}$, and channel gating kinetics, i.e. the cooperativity parameters (cooperativity percentage $p$ and coupling strength $K J$ ), in a compartment were altered systematically to investigate their impact on the site of initiation.

The results indicated that the only factor that affected the site of initiation is the channel distribution (Figure 9.10 and 9.8) .

\subsection{Action Potential Propagation and Conduction Velocity}

The orthodromic and antidromic propagation of action potentials were analyzed using the first action potential onset latencies $\left(\Delta t_{\text {onset }}\right)$ or peak latencies $\left(\Delta t_{\text {peak }}\right)$. The conduction velocities were estimated from the plots of onset latency versus the distance that the action potential travels on both directions.

The conduction velocity was highly sensitive to the changes in the parameters given above.

A trivial prediction for the effect of $r_{l}$ on the conduction velocity would be that as the $r_{l}$ increases, the conduction velocity should decrease. This prediction was approved by the simulation results (Figure 9.8). For the non-cooperative model, the conduction velocity for $r_{l}=3 \mathrm{~K} \Omega . \mathrm{mm}$ was $0.9 \mathrm{~m} / \mathrm{s}$ and for $r_{l}=5 \mathrm{~K} \Omega . \mathrm{mm}$ was $0.5 \mathrm{~m} / \mathrm{s}$.

The cooperativity parameters $(p$ and $K J)$ were surprisingly ineffective on the conduction velocity for $r_{l}=3 \mathrm{~K} \Omega$.mm. However, the coupling strength had a clear impact on the somatic onset latency and a slight impact on the conduction velocity for $r_{l}=5 \mathrm{~K} \Omega . \mathrm{mm}$.

The changes in the $\mathrm{Na}^{+}$channel density of soma $\left(g_{\text {soma }}\right.$, AIS $\left(g_{A I S}\right)$ and NR $\left(g_{N R}\right)$ also affected the conduction velocity both at $r_{l}=3 \mathrm{~K} \Omega . \mathrm{mm}$ (Figure 9.10) and at $r_{l}$ $=5 \mathrm{~K} \Omega . \mathrm{mm}$. The results obtained for different combinations of channel densities are given in Table 9.3 and 9.4. 

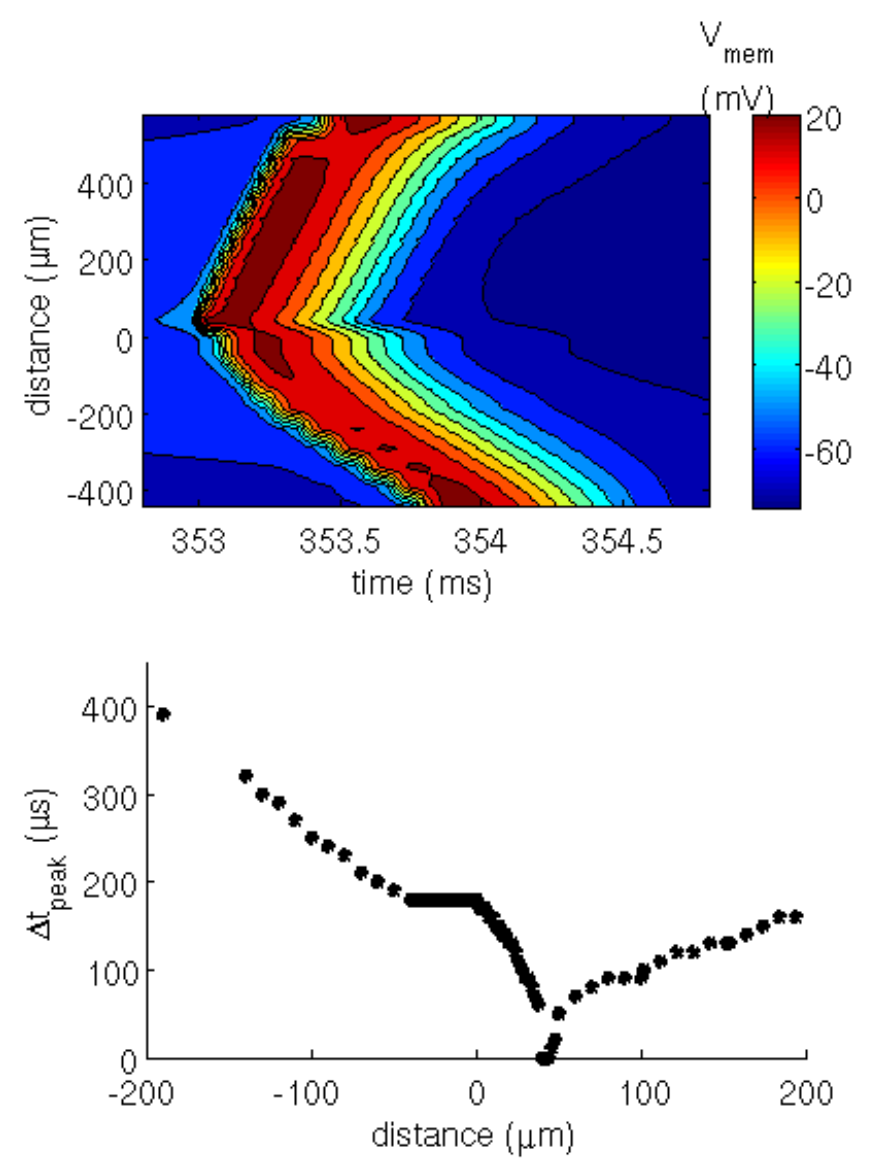

Figure 9.6: Action potential initiation and propagation. Upper: The contour plot for the propagation of an action potential on MCCAIS model. Lower: The onset latency-distance plot, that was obtained from the same simulation. The somatic onset latency is around $200 \mu \mathrm{s}$, which is in agreement with previous studies (Palmer and Stuart [139]; Kole et al. [97]; Hu et al. [86]). A constant current was injected at the soma to produce a steady-state firing rate $\nu_{0}=10 \mathrm{~Hz} . g_{A I S}=g_{N R}=10^{*} g_{\text {soma }}=3000$ $\mathrm{pS} / \mu \mathrm{m}^{2}, r_{L}=5 \mathrm{~K} \Omega . \mathrm{mm}$. The model was non-cooperative, and dendritic conductances were set as same with soma. 


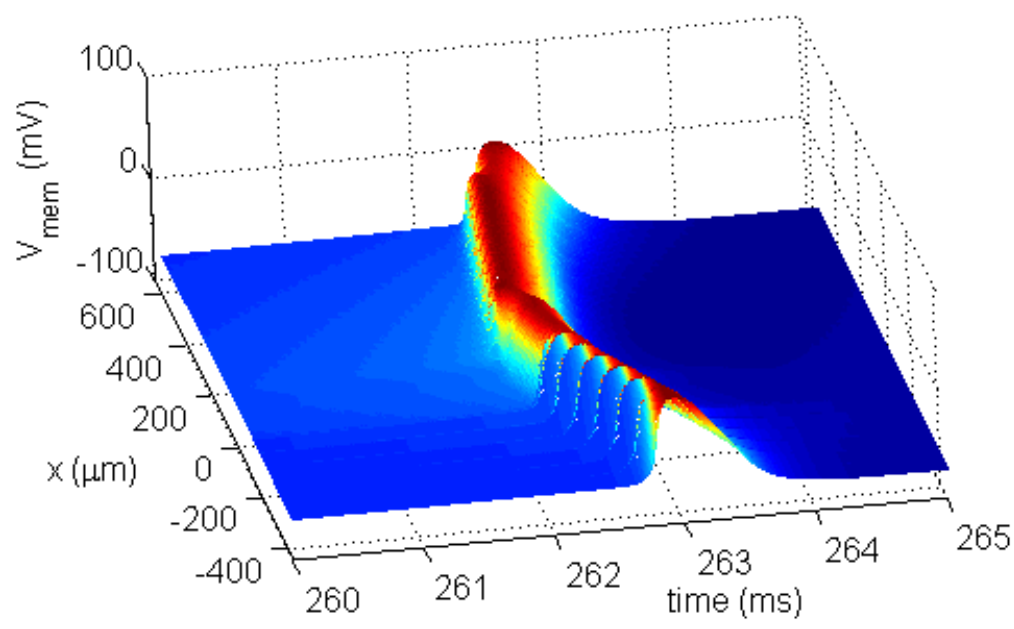

Figure 9.7: Action potential initiation and propagation in 3D.A constant current was injected at the soma to produce a steady-state firing rate $\nu_{0}=10 \mathrm{~Hz} . g_{A I S}=$ $g_{N R}=10^{*} g_{\text {soma }}=3000 \mathrm{pS} / \mu \mathrm{m}^{2}, r_{L}=5 \mathrm{~K} \Omega . \mathrm{mm}$. The model was non-cooperative, and dendritic conductances were set as same with soma. The seemingly saltatory movement at the dendrites stem from increased compartment length $(50 \mu \mathrm{m})$.

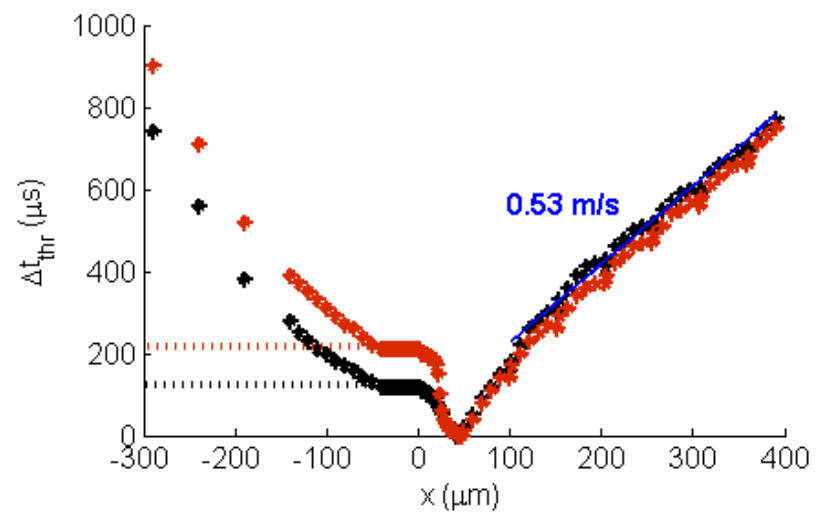

Figure 9.8: AP propagation in MCCAIS model for non-cooperative and cooperative model. The light colored dots represent the numerical results. These results were fit using a linear function of latency and distance (solid blue line) and the conduction velocity of each case was estimated from the slopes of the fitting functions (as given next to the solid line). The conduction velocities for both cases were the same.Red : cooperative $(p=10 \%, K J=400 \mathrm{mV})$ and $r_{L}=5 \mathrm{~K} \Omega . \mathrm{mm}$; black : non-cooperative and $r_{L}=5 \mathrm{~K} \Omega . \mathrm{mm}$. A noisy current with $\tau_{I}=5 \mathrm{~ms}$ and an automatically adjusting $\mu_{I}$ was injected to maintain $\nu_{0}=10 \mathrm{~Hz}$. $\sigma_{I}$ was set to give $\sigma_{V} \cong 5 \mathrm{mV}$. 

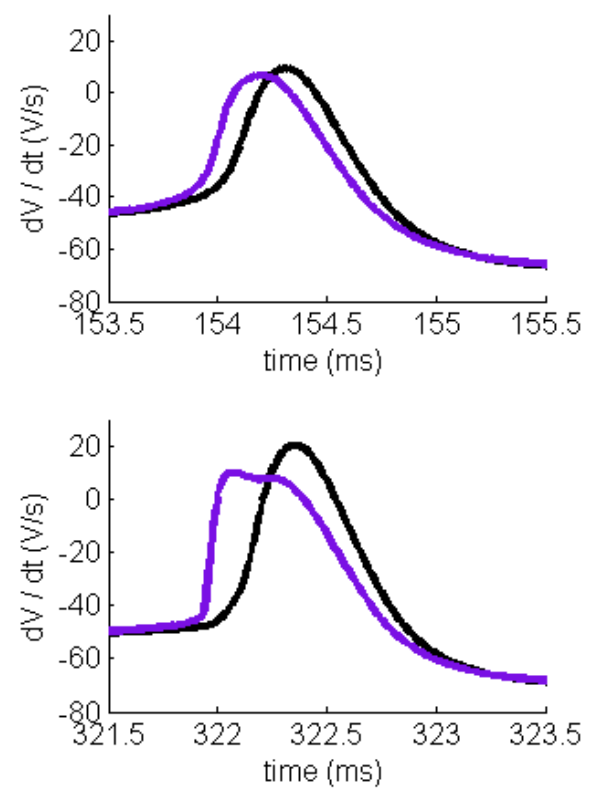

Figure 9.9: AP waveforms for Figure 9.8. Upper: non-cooperative model with $r_{l}=5 \mathrm{~K} \Omega . \mathrm{mm}$; lower : cooperative model with $r_{l}=5 \mathrm{~K} \Omega . \mathrm{mm}, p=10 \%, K J=400$ $\mathrm{mV}$. Blue: AIS, black : soma. A noisy current with $\tau_{I}=5 \mathrm{~ms}$ and an automatically adjusting $\mu_{I}$ was injected to maintain $\nu_{0}=10 \mathrm{~Hz}$. $\sigma_{I}$ was set to give $\sigma_{V} \cong 5 \mathrm{mV}$.

\begin{tabular}{|c||c|c|}
\hline Channel Density & $\mathbf{g}_{N R}=\mathbf{g}_{\text {soma }}$ & $\mathbf{g}_{N R}=\mathbf{3}^{*} g_{\text {soma }}$ \\
\hline \hline $\mathbf{g}_{A I S}=\mathbf{g}_{\text {soma }}$ & $0.48 \mathrm{~m} / \mathrm{s}$ & $0.48 \mathrm{~m} / \mathrm{s}$ \\
\hline $\mathbf{g}_{A I S}=\mathbf{3}^{*} \mathbf{g}_{\text {soma }}$ & $1 \mathrm{~m} / \mathrm{s}$ & $0.9 \mathrm{~m} / \mathrm{s}$ \\
\hline
\end{tabular}

Table 9.3: Axonal conduction velocities with respect to channel densities in AIS and NR. The axonal conduction velocities were estimated with the slope of increase in the onset latency between $x_{1}=102 \mu \mathrm{m}$ and $x_{2}=362 \mu \mathrm{m}$ (Figure 9.10) for the combinations of channel densities in AIS and NR. The model was non-cooperative. A noisy current with $\tau_{I}=5 \mathrm{~ms}$ and an automatically adjusting $\mu_{I}$ was injected to maintain $\nu_{0}=10 \mathrm{~Hz}$. $\sigma_{I}$ was set to give $\sigma_{V} \cong 5 \mathrm{mV}$. 


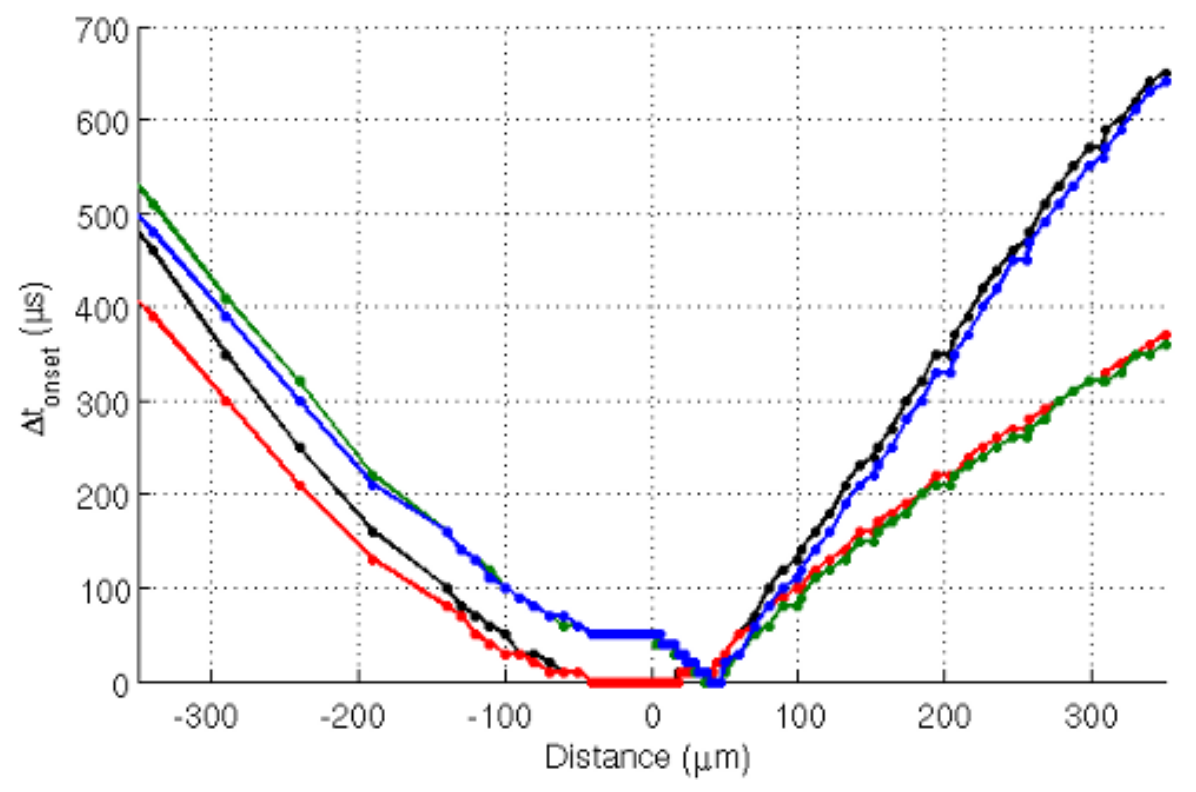

Figure 9.10: AP propagation in MCCAIS model for varying channel distributions in non-cooperative MCCAIS model. The AP onset latencies, for different combinations of channel densities in soma, AIS and NR, are depicted as function of distance, $x$ at $r_{L}=3 \mathrm{~K} \Omega$.mm. A noisy current with $\tau_{I}=5 \mathrm{~ms}$ and an automatically adjusting $\mu_{I}$ was injected to maintain $\nu_{0}=10 \mathrm{~Hz}$. $\sigma_{I}$ was set to give $\sigma_{V} \cong 5 \mathrm{mV}$.

\begin{tabular}{|c||c|c|}
\hline Channel Density & $\mathbf{g}_{N R}=\mathbf{g}_{\text {soma }}$ & $\mathbf{g}_{N R}=\mathbf{3}^{*} g_{\text {soma }}$ \\
\hline \hline $\mathbf{g}_{A I S}=\mathbf{g}_{\text {soma }}$ & $0.58 \mathrm{~m} / \mathrm{s}$ & $0.63 \mathrm{~m} / \mathrm{s}$ \\
\hline $\mathbf{g}_{A I S}=\mathbf{3}^{*} \mathbf{g}_{\text {soma }}$ & $0.67 \mathrm{~m} / \mathrm{s}$ & $0.56 \mathrm{~m} / \mathrm{s}$ \\
\hline
\end{tabular}

Table 9.4: Dendritic conduction velocities with respect to channel densities in AIS and NR. The dendritic conduction velocities were estimated with the slope of decrease in the onset latency between $x_{1}=-400 \mu \mathrm{m}$ and $x_{2}=-100 \mu \mathrm{m}$ (Figure 9.10) for the combinations of channel densities in AIS and NR. The model was noncooperative. A noisy current with $\tau_{I}=5 \mathrm{~ms}$ and an automatically adjusting $\mu_{I}$ was injected to maintain $\nu_{0}=10 \mathrm{~Hz}$. $\sigma_{I}$ was set to give $\sigma_{V} \cong 5 \mathrm{mV}$. 


\subsection{Onset Rapidness of Axonal and Somatic Action Potentials}

The AP waveforms (Figure 9.2) of MCCAIS model clearly indicated the difference in $\Gamma$ between canonical and cooperative models : In cooperative model the onset grows piecewise-linearly ("kinky onset"), opposite to the canonical models, where the onset is growing exponentially ("smooth onset"). The phase plots also revealed this pattern (Figure 9.3). To obtain quantitative results, $\Gamma$ was defined as the phase plot slope at $\dot{V}\left(x, t_{i}\right)>10 \mathrm{mV} / \mathrm{ms}$ and was obtained for increasing values of cooperativity parameters (coupling strength, $K J$, and cooperativity percentage, $p$ ). The results (Figure 9.11) indicated an increasing onset rapidness with increasing coupling strength between $x=-200 \mu \mathrm{m}$ and $x=150 \mu \mathrm{m}$. $\Gamma$ as a function of $x$ displayed two peak points one closer to or in the soma (somatic peak) and one in the axon (axonal peak). As the coupling strength increased both peaks moved closer to the AIS, where the cooperative channel gating was implemented. Additionally, the axonal peaks were higher than somatic peaks when $K J<800 \mathrm{mV}$. After this value, the somatic peak shifted to AIS and exceeded the axonal peak $\left(\Delta \cong 35 \mathrm{~ms}^{-1}\right)$. The spatial shift of the maximum $\Gamma$ towards AIS was not affected by the cooperativity percentage $p$ (Figure 9.12). On the other hand, it was linearly dependent on $K J$ (shift rate $=0.14 \mu \mathrm{m} / \mathrm{mV}$ ).

\subsection{Linear Response in MCCAIS Model}

The realistic neuron models provides researchers the opportunity to connect microscopic events (e.g. ionic channel gating) to macroscopic events (e.g. population coding). Additionally, MCCAIS model provides a valuable tool to test an alternative hypothesis for channel gating kinetics and to interpret its possible effects on population level response properties, by investigating the effect of implementing cooperative channel gating on the individual neural response.

\subsubsection{Stationary Response to Current Injection}

The frequency-current (f-I) curves for constant and noisy current injection were given in Figure 9.13. The subthreshold fluctuations due to noisy current injection is likely the explanation for the offset of this curve, as these fluctuations lead to a steadystate firing rate and they keep the membrane potential very close to the threshold to enable fast responses. Both curves represent a typical type-I neuron model, as predicted from Wang-Buzsaki model. 
142 Chapter 9: Multi-compartmental Cooperative Axon Initial Segment (MCCAIS) Model

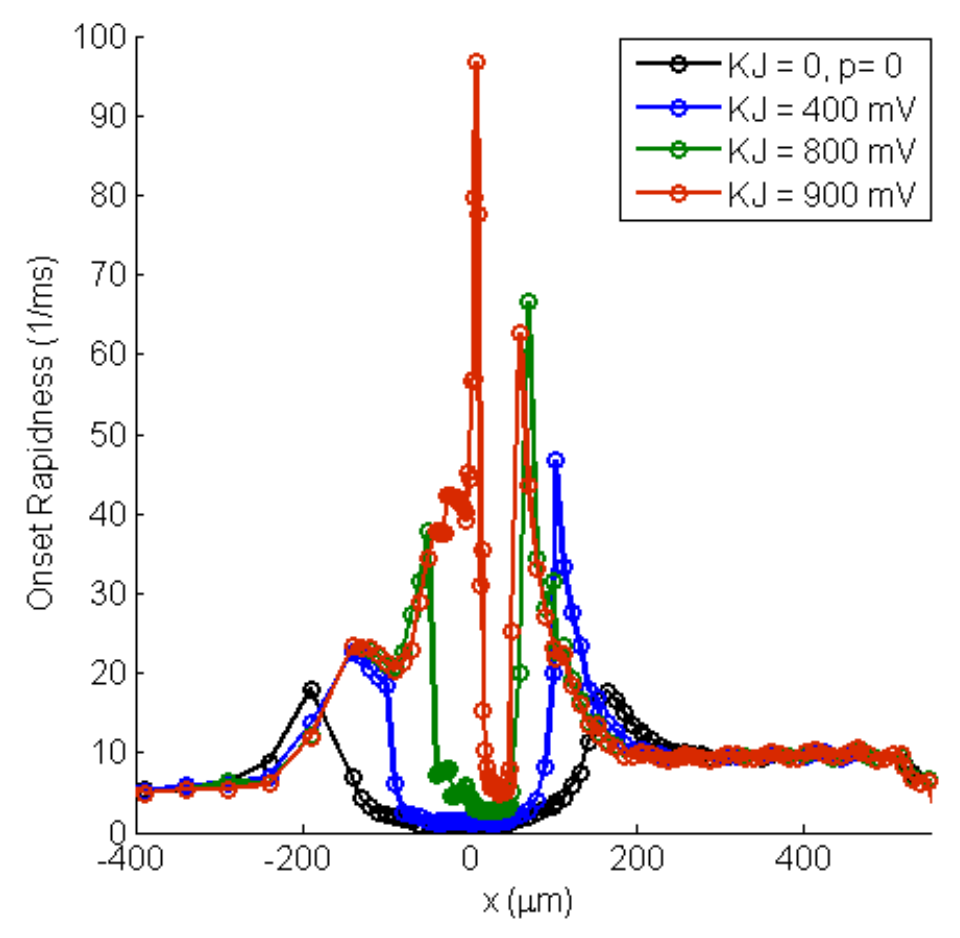

Figure 9.11: Onset Rapidness, $\Gamma$, as a function $x . g_{A I S}=g_{N R}=3^{*} g_{\text {soma }}=$ $3^{*} g_{\text {dend }}$. A noisy current with $\sigma_{I}=, \tau_{I}=5 \mathrm{~ms}$ and an automatically adjusted $\mu_{I}$ was injected to maintain $\nu_{0}=10 \mathrm{~Hz} \cdot p=10 \%$. 


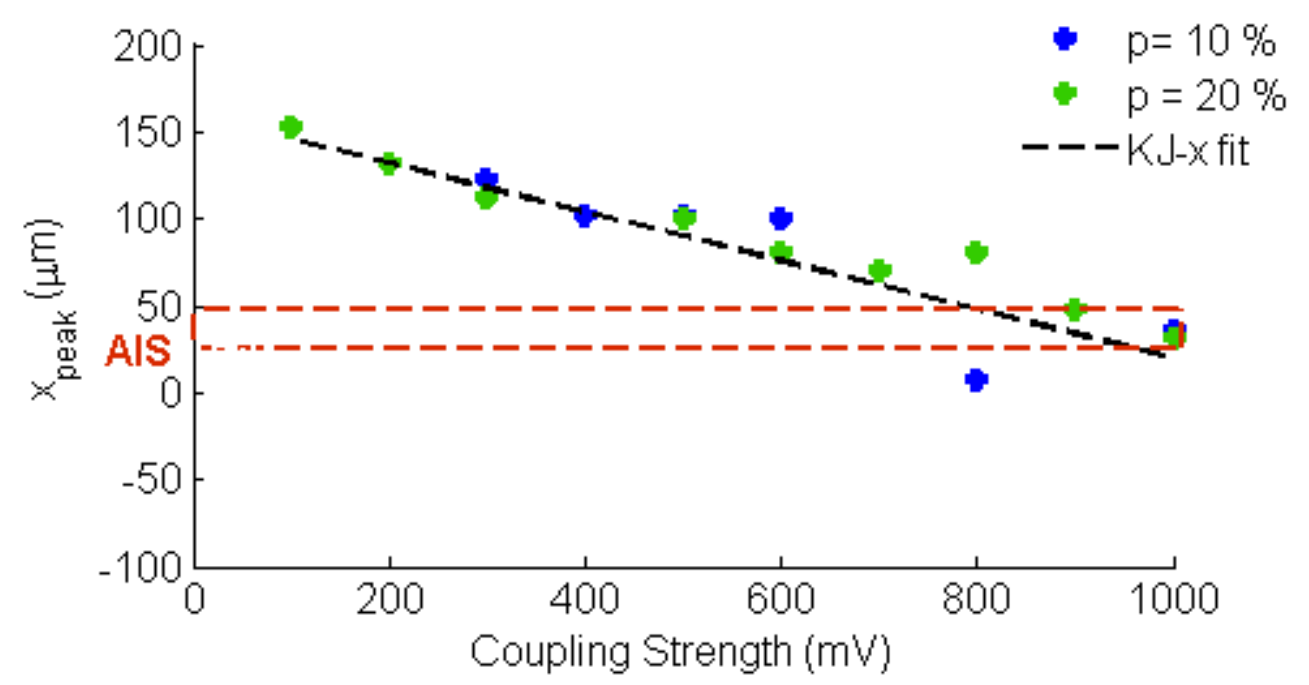

Figure 9.12: The location of maximum $\Gamma$ as a function $K J$. The red dashed lines indicate the interval that corresponds to AIS. The spatial shift of maximum $\Gamma$ is linearly dependent on $K J$ and the slope of the fit line is same for both $p=10 \%$ and $p=20 \% . g_{A I S}=g_{N R}=3^{*} g_{\text {soma }}=3^{*} g_{\text {dend }}$. A noisy current with $\sigma_{I}=, \tau_{I}=5$ ms and an automatically adjusted $\mu_{I}$ was injected to maintain $\nu_{0}=10 \mathrm{~Hz}$.

\subsection{Summary and Discussion}

The MCCAIS model was first tested for the parameters without the contribution of cooperativity, such as AP propagation and conduction velocity, AP waveform and onset rapidness and the effects of spatially varying $\mathrm{Na}^{+}$channel densities. Only after the characterization of the non-cooperative model, the contribution of cooperative channel gating was investigated and was compared to the non-cooperative case.

The findings of the MCCAIS model emphasize some intriguing points. First of all, the classical Hodgkin-Huxley type model failed to reproduce the "kinky" onset that was frequently observed in cortical neurons (Naundorf et al. [130], [132]; Volgushev et al. [182]; Bean [15]; Baranauskas et al. [14]). This result was also previously reported by Naundorf et al. [132] and in this study, the contribution of cooperative gating kinetics in AIS was suggested as a possible mechanism to reproduce the cortical AP onset and threshold variability. This suggestion is opposed by the argument that the rapid AP onset in cortical neurons can be explained well with the backpropagation of axonal APs (McCormick et al. [123]; Shu et al. [164]; Kole et al. [97]; $\mathrm{Yu}$ et al. [187]). The flaws in these arguments have been recently discussed by the Naundorf et al. [133] and Baranauskas et al. [14]. Especially, the measurements that are performed on the axonal cut ends or "blebs" are not reliable enough to exclude 


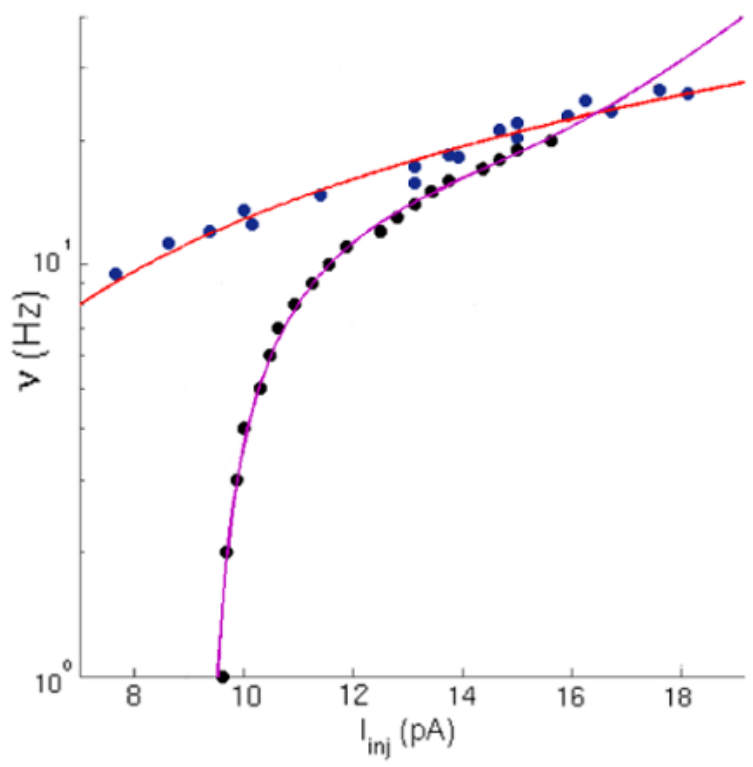

Figure 9.13: The Frequency-Current (f-I) relationship for non-cooperative MCCAIS model. The f-I curves were obtained under noisy (blue dots) and constant (black dots) current injection. Under noisy current injection, f-I curve started with an offset (see text). The model indicated a Type-I firing pattern as expected from a modified Wang-Buzsaki model. The numerical results were further fit either with a simple linear function (red solid line) or with a cubic polynomial function (purple solid line). Both predicted good at low frequency values $(p<0.05)$. 
a possibility for inter-channel coupling (McCormick et al. [123]). The arguments are discussed in more detail in section 9.7.1. The MCCAIS model simulation results indicated that the cooperative model indeed reproduces the rapid AP onsets, i.e. without the need of unrealistically high $\mathrm{Na}^{+}$channel densities or a distortion in the actual neuron geometry (Mainen et al. [117]; McCormick et al. [123]).

To sum up, the results mentioned in this chapter indicate that the replacement of classical channel gating kinetics with cooperative gating kinetics results in the generation of APs with very rapid onsets in response to noisy inputs. The fact that this propeerty is one of the critical characteristics that are also observed for cortical neurons and that can so far not be reproduced by using classical gating and realistic channel densities, supports the hypothesis that cooperative channel gating can indeed be a key element of the AP generating mechanisms located in AIS. The second critical characteristic of cortical neurons in vitro is the high cut-off frequency, which is also indirectly related to the onset rapidness as mentioned before. Therefore, the next step of the research should be the investigation of this relationship.

\subsubsection{A detailed look to the counter-arguments}

Although the cooperative $\mathrm{Na}^{+}$channel gating hypothesis lacks direct biological evidence, there is also no direct evidence to disprove the hypothesis. The most important examples of inter-channel coupling in clusters of ionic channels were mentioned in section 7.1. It is clearly an open question whether this level of interaction is possible in a neuronal segment, i.e. in the AIS. That is yet to be investigated. However, the studies claiming that there is no need to look for alternative explanations instead of canonical Hodgkin-Huxley type formulations (McCormick et al. [123]) actually provided more evidence that there is a need to look for other plausible models to explain the dynamics of cortical AP generators (Naundorf et al. [133]; McCormick et al. [123]). Furthermore, their Hodgkin-Huxley type model included unrealistically high $\mathrm{Na}^{+}$channel density, opposing to what is reported experimentally (Baranauskas and Martina [13], Colbert and Pan [39], Fleidervish et al. [60]), and yet did not completely fit with their results.

The use of blebs as the site of axonal recordings, although it has become common in the literature on AP initiation and propagation (McCormick et al. [123]; Shu et al. [164]), is not reliable to interpret the real $\mathrm{Na}^{+}$channel densities or interactions. It is well-known that, in the case of such injuries, the axonal cytoskeleton rearranges itself (Schafer et al. [157]). This is a very crucial point, since the cytoskeletal proteins, especially of the ankyrin and spectrin families, is known to be important for the recruitment and localization of the ionic channels on the neuronal membrane (Angelides et al. [7];Pan et al. [140],Grubb and Burrone [72];Boiko et al. [18];Duflocq et al. [51];Ogawa and Rasband [136];Hedstrom et al. [76];Dzhashiashvili et al. [52]). Indeed, 
it was shown that neuronal injury can cause rapid and irreversible proteolysis of the AIS cytoskeleton and loss of ion channel clusters (Schafer et al. [157]). It is, then, obvious that the studies performed on such blebs can be useful for many purposes but not for investigating the possible contribution of inter-channel coupling. Also for this reason, the probability of cooperative channel gating can not be excluded with the findings obtained by this experimental design. Another important aspect is that axonal cuts or injuries trigger cellular mechanisms that cope with infections (Schafer et al. [157]). Under these circumstances, it should not be claimed that the results are obtained under healthy physiological conditions and should not be compared to the ones that are obtained by comparably intact cellular morphology.

\subsubsection{Perspectives from MCCAIS model}

The onset rapidness of action potentials strictly depend on the $\mathrm{Na}_{V}$ channel gating kinetics. Between the cooperativity parameters, the coupling strength exhibited stronger impact on onset rapidness for small coupling percentages.

The models that were used to investigate the cooperativity hypothesis do not provide direct hints about the underlying mechanisms of inter-channel coupling. However, among other possibilites, the special cytoskeletal structure of the AIS may serve as a network that connect $\mathrm{Na}_{V}$ channels and, therefore, may create the grounds for cooperative activity. Further experimental investigation is needed to prove this hypothesis.

The results obtained so far provide an idea about how cooperative gating kinetics could contribute to individual neuron response. However, in the simulations described in this chapter, only realistic channel densities (lower than 10-fold of soma) were used. Therefore, another aspected that should be tested is the effect of extremely high channel densities. Additionally, the implementation of some other ionic channels that came to the focus in the recent years, e.g. the $\mathrm{K}_{V} 1$ channels in the AIS (Kole et al. [98]; Lorincz and Nusser [112]; Inda et al. [90]), could provide new insights. 


\section{Part IV}

\section{Summary and Perspective}



In this thesis, I investigated two unique and critical properties of action potentials of cortical neurons: the threshold variability and the rapid onset. I performed simulations on three different models for this purpose.

In chapter 6 , the focus was mainly to test an analytical method that can provide information about the changes in the somatic membrane potential at the time of threshold crossing at the axonal initiation site, independent from the chosen threshold value. For this purpose, I presented a modified conductance-based Ball-and-Stick model (Rall [144]) and a statistical framework to analyze the somatic membrane potential fluctuations with respect to the potential changes at a given axonal compartment. Furthermore, I compared my numerical results with the an analytical framework (Wei [184]). My results indicated three points: First, the somatic and axonal potentials at the action potential onset do not differ significantly if the distance from soma $x$ is less than $100 \mu \mathrm{m}$. Second, the spike-triggered average was linearly dependent on the choice of the threshold. On the other hand, spike triggered variance was independent from the threshold and was virtually invariant for compartments less than $100 \mu \mathrm{m}$ away from soma. Considering that the action potential initiation site is in the range of $30-40 \mu \mathrm{m}$ in most cortical neurons, spike-triggered variance can be a valuable indirect tool to interpret action potential generation dynamics at an axonal site using somatic voltage statistics. Secondly, my results on the partially active ball-and-stick threshold model indicated that even $35 \mu \mathrm{m}$ distance was not far enough to generate a significant difference in the dynamic response, e.g. in the cut-off frequency. A recent paper reported interesting findings about the adjustment of neuronal excitability by relocation of AIS either more proximal or more distal to soma (Grubb and Burrone [71]). In this study, it was claimed that even very small distances such as $5 \mu \mathrm{m}$ could have a significant effect on the neuronal response and the firing rate. However, a reorganization of molecular interactions in the AIS was not investigated. Therefore, this study does not provide enough proof for the hypothesis that the repositioning of the AIS is the origin of the observed effect. The results of my simulations also confirm that the repositioning alone is unlikely to be the explanation.

In chapter 8 , I further characterized a previously constructed single-compartment conductance-based model (Huang et al. [89]) with cooperative $\mathrm{Na}^{+}$channel gating kinetics, to provide grounds for comparison with a following more sophisticated multicompartmental model. This simple single-compartment model was a useful tool to understand the possible outcomes of the cooperativity hypothesis in a restricted single segment. My results independently confirmed the validity of several predictions of cooperative channel gating hypothesis by proving that the implementation of such gating kinetics instead of canonical Hodgkin-Huxley type kinetics can indeed reproduce the rapid action potential onsets as observed in cortical neurons (Huang et al. [89]; Naundorf et al. [132]). 
In chapter 9 , I presented a multi-compartmental conductance-based model, with realistic morphological features and cooperative gating kinetics at the axon initial segment. Using this model, I was able to show the contribution of cooperative gating kinetics in a more realistic morphology. The results from this model also further confirmed that cooperative channel gating might be involved in the cortical action potential generating mechanisms, that are located in axon initial segment.

The last two chapters provided information on the possible outcomes of the implementation of cooperative $\mathrm{Na}^{+}$channel gating in the action potential generating mechanisms. The results of the simulations indicated that the effective coupling strength is a significant factor for the determination of activation threshold and of the duration that a channel remains open, due to the changes in the opening and closing rates, as also mentioned in previous studies (Huang et al. [89]; Undrovinas et al. [179]).

The results mentioned in chapter 8 and 9 pointed out the strong dependence of onset rapidness on the value of coupling strength $(K J)$. On the other hand, the cooperativity percentage $(p)$ did not play a significant role as long as $p<30 \%$ and $K J$ $<400 \mathrm{mV}$. The classical Hodgkin-Huxley type model failed to reproduce the "kinky" onset that was frequently observed in cortical neurons (Naundorf et al. [130], [132]; Volgushev et al. [182]; Bean [15]; Baranauskas et al. [14]) and that was reproduced by the cooperative model. The results, therefore, implied that the cooperative gating kinetics in the AIS can be a possible mechanism to reproduce the cortical AP onset and threshold variability.

What would be the importance of cooperativity for a neuronal population? The functional relevance of the cooperative gating hypothesis for the population response stems from its possible role in the action potential generating mechanisms. As shown earlier, the individual neuronal response, i.e. the cut-off frequency, exhibits a direct relation with the action potential onset rapidness (Fourcaud-Trocme et al. [63], Naundorf et al. [130], Wei and Wolf [185]). In other terms, high cut-off frequencies are likely to be achieved with rapid onsets. The importance of cooperative gating becomes clear when taken together with the results mentioned in this thesis : if the cooperative gating is leading to rapid onsets, then it must also lead to higher cut-off frequencies. Therefore, the most urgent step following this research should be in examining this relationship with the current methods.

What would high cut-off frequencies imply? The cut-off frequency, by definition, gives the critical frequency after which the ability of neuron to respond the fast-changing time-varying input declines sharply. Therefore, high cut-off frequency implies that the neuron can detect a fast oscillation in the input and respond by locking its response phase to the phase presented in the stimulus. Cortical neurons exhibit 
a cut-off frequency at least 10 -fold higher than their average firing rate. This means that the cortical neurons are able to detect and respond the very rapid oscillations in the input they are exposed to. This property is crucial when considering population response that usually requires the detection of the signals in the micro-second scale. Therefore, if the cooperative gating kinetics can reproduce the high onset rapidness and also the high cut-off frequencies, the possible contribution of such kinetics in the action potential generating mechanisms should not be ignored.

The key questions and answers of this thesis provide various suggestions for future studies. For instance, the use of spike-triggered variance as a statistical tool to gather information from soma about the threshold crossing at an axonal site still requires further testing. One interesting and computationally cheap method would be using the already available models, such as the one mentioned in chapter 9 , to investigate the maximum distance that would still have similar potential with soma at the action potential onset. In addition to this, the cooperativity hypothesis clearly presents a new perspective on the impact of channel gating kinetics on neuronal function. Still, there are many open questions about this hypothesis. For instance, the direct biological evidence of cooperativity in axon initial segment is still missing. Also, another intriguing aspect of the subject would be to prove that it is also energetically plausible to have cooperative kinetics in the axon initial segment. In my simulations, I only investigated one type of dynamic stimulation, which was the sinusoidally oscillating current injection. Other methods are also mentioned in the previous studies (Silberberg et al. [166];Boucsein et al. [20]) and would be interesting to study these type of stimulations, especially with the multi-compartmental cooperative axon initial segment model. In summary, the project and the results present many points that can be investigated further to get one step closer to the complete understanding of the relation between the properties of action potential generating mechanisms and the neural transfer functions.

In conclusion, my opinion about the results of my thesis project is that they provide only introductory steps to new horizons towards the ultimate goal of understanding how the brain operates. 


\section{Bibliography}

[1] Ahumada, G., Bergmann, S., Carlson, E., Corr, P., and Sobel, B. (1979). Augmentation of cyclic amp content induced by lysophosphatidyl choline in rabbit hearts. Cardiovasc Res, 13(7):377-382.

[2] Alle, H., Roth, A., and Geiger, J. (2009). Energy-efficient action potentials in hippocampal mossy fibers. Science, 325(5946):1405-1408.

[3] Allen, C. and Stevens, C. (1994). An evaluation of causes for unreliability of synaptic transmission. Proc Nat Acad Sci, 91:10380-10383.

[4] Almers, W. and Stirling, C. (1984). Distribution of transport proteins over animal cell membranes. J Membr Biol, 77(3):169-186.

[5] Anderson, J., Lampl, I., Gillespie, D., and Ferster, D. (2000). The contribution of noise to contrast invariance of orientation tuning in cat visual cortex. Science, 290(5498):1968-1972.

[6] Andreasen, M. and Lambert, J. (1999). Somatic amplification of distally generated subthreshold epsps in rat hippocampal pyramidal neurones. J Physiol, 519:85-100.

[7] Angelides, K., Elmer, L., Loftus, D., and Elson, E. (1988). Distribution and lateral mobility of voltage-dependent sodium channels in neurons. J Cell Biol, 106(6):1911-1925.

[8] Angelides, K. and Nutter, T. (1984). Molecular and cellular mapping of the voltage-dependent na channel. Biophys J, 45(1):31-34.

[9] Araki, T. and Otani, T. (1955). Response of single motoneurons to direct stimulation in toad's spinal cord. J Neurophysiol, 18(5):472-485.

[10] Arsiero, M., Lüscher, H., Lundstrom, B., and Giugliano, M. (2007). The impact of input fluctuations on the frequency-current relationships of layer 5 pyramidal neurons in the rat medial prefrontal cortex. J Neurosci, 27(12):3274-3284.

[11] Astman, N., Gutnick, M., and Fleidervish, I. (2006). Persistent sodium current in layer 5 neocortical neurons is primarily generated in the proximal axon. $J$ Neurosci, 26(13):3465-3473.

[12] Attwell, D. and Laughlin, S. (2001). An energy budget for signaling in the grey matter of the brain. J Cereb Blood Flow Metab, 21:1133-1145.

[13] Baranauskas, G. and Martina, M. (2006). Sodium currents activate without a hodgkin-and-huxley-type delay in central mammalian neurons. J Neurosci, 26:671684 . 
[14] Baranauskas, G., Mukovskiy, A., Wolf, F., and Volgushev, M. (2010). The determinants of the onset dynamics of action potentials in a computational model. Neuroscience, 167(4):1070-1090.

[15] Bean, B. (2007). The action potential in mammalian central neurons. Nat Rev Neurosci, 8:451-465.

[16] Bender, K. and Trussell, L. (2009). Axon initial segment ca ${ }^{2+}$ channels influence action potential generation and timing. Neuron, 61(2):259-271.

[17] Bishop, P. (1953). Synaptic transmission; an analysis of the electrical activity of the lateral geniculate nucleus in the cat after optic nerve stimulation. Proc $R$ Soc Lond B Biol Sci, 141(904):362-392.

[18] Boiko, T., Van Wart, A., Caldwell, J., Levinson, S., Trimmer, J., and Matthews, G. (2003). Functional specialization of the axon initial segment by isoform-specific sodium channel targeting. J Neurosci, 23(6):2306-2313.

[19] Borg-Graham, L., Monier, C., and Frègnac, Y. (1998). Visual input evokes transient and strong shunting inhibition in visual cortical neurons. Nature, 393(6683):369-373.

[20] Boucsein, C., Tetzlaff, T., Meier, R., Aertsen, A., and Naundorf, B. (2009). Dynamical response properties of neocortical neuron ensembles: multiplicative versus additive noise. J Neurosci, 29(4):1006-1010.

[21] Boyden, E., Zhang, F., Bamberg, E., Nagel, G., and Deisseroth, K. (2005). Millisecond-timescale, genetically targeted optical control of neural activity. Nat Neurosci, 8(9):1263-1268.

[22] Brunel, N. (2000). Dynamics of sparsely connected networks of excitatory and inhibitory spiking neurons. J Comput Neurosci, 8(3):183-208.

[23] Brunel, N., Chance, F., Fourcaud, N., and Abbott, L. (2001). Effects of synaptic noise and filtering on the frequency response of spiking neurons. Phys Rev Lett, $86: 2186-2189$.

[24] Brunel, N., Frègnac, Y., Meunier, C., and Nadal, J. (2003). Neuroscience and computation. J Physiol Paris, 97(4-6):387-390.

[25] Brunel, N. and Hakim, V. (1999). Fast global oscillations in networks of integrateand-fire neurons with low firing rates. Neural Comput, 11(7):1621-1671.

[26] Brunel, N. and Latham, P. (2003). Firing rate of the noisy quadratic integrateand-fire neuron. Neural Comput, 15(10):2281-2306. 
[27] Brunel, N. and Sergi, S. (1998). Firing frequency of leaky intergrate-and-fire neurons with synaptic current dynamics. J Theor Biol, 195(1).

[28] Burak, Y., Lewallen, S., and Sompolinsky, H. (2009). Stimulus-dependent correlations in threshold-crossing spiking neurons. Neural Comput, 21(8):2269-2308.

[29] Buzsaki, G. and Draguhn, A. (2004). Neuronal oscillations in cortical networks. Science, 304:1926-1929.

[30] Caldwell, J., Schaller, K., Lasher, R., Peles, E., and Levinson, S. (2000). Sodium channel na(v)1.6 is localized at nodes of ranvier, dendrites, and synapses. Proc Natl Acad Sci U S A., 97(10):5616-5620.

[31] Cantiello, H., Stow, J., Prat, A., and Ausiello, D. (1991). Actin filaments regulate epithelial na ${ }^{+}$channel activity. Am J Physiol, 261(5 Pt 1):C882-C888.

[32] Cantrell, A. and Catterall, W. (2001). Neuromodulation of na ${ }^{+}$channels: an unexpected form of cellular plasticity. Nat Rev Neurosci, 2(6):397-407.

[33] Carandini, M., Mechler, F., Leonard, C., and Movshon, J. (1996). Spike train encoding by regular-spiking cells of the visual cortex. J Neurophysiol, 76(5):34253441.

[34] Carter, B. and Bean, B. (2009). Sodium entry during action potentials of mammalian neurons: incomplete inactivation and reduced metabolic efficiency in fastspiking neurons. Neuron, 64(6):898-909.

[35] Catterall, W. (1981). Localization of sodium channels in cultured neural cells. $J$ Neurosci., 1(7):777-783.

[36] Chance, F., Abbott, L., and Reyes, A. (2002). Gain modulation from background synaptic input. Neuron, 35(4):773-782.

[37] Clark, B., Goldberg, E., and Rudy, B. (2009). Electrogenic tuning of the axon initial segment. Neuroscientist, 15(6):651-68.

[38] Colbert, C. and Johnston, D. (1996). Axonal action-potential initiation and $\mathrm{na}^{+}$channel densities in the soma and axon initial segment of subicular pyramidal neurons. J Neurosci, 16(21):6676-6686.

[39] Colbert, C. and Pan, E. (2002). Ion channel properties underlying axonal action potential initiation in pyramidal neurons. Nat Neurosci, 5:533-538.

[40] Connor, J. and Stevens, C. (1971a). Inward and delayed outward membrane currents in isolated neural somata under voltage clamp. J Physiol, 213(1):1-19. 
[41] Connor, J. and Stevens, C. (1971b). Voltage clamp studies of a transient outward membrane current in gastropod neural somata. J Physiol, 213(1):21-30.

[42] Connors, B. and Gutnick, M. (1990). Intrinsic firing patterns of diverse neocortical neurons. Trends Neurosci, 13(3):99-104.

[43] Conradi, S. (1966). Ultrastructural specialization of the initial axon segment of cat lumbar motoneurons. preliminary observations. Acta Soc Med Ups, 71):281284.

[44] Coombs, J., Curtis, D., and Eccles, J. (1957a). The generation of impulses in motoneurones. J Physiol, 139(2):232-249.

[45] Coombs, J., Curtis, D., and Eccles, J. (1957b). The interpretation of spike potentials of motoneurones. J Physiol, 139(2):198-231.

[46] Crotty, P., Sangrey, T., and Levy, W. (2006). Metabolic energy cost of action potential velocity. J Neurophysiol, 96(3):1237-1246.

[47] Dayan, P. and Abbott, L. F. (2001). Theoretical neuroscience : Computational and mathematical modeling of neural systems. MIT Press, Cambridge, England.

[48] Dekker, J. and Yellen, G. (2006). Cooperative gating between single hon pacemaker channels. J Gen Physiol, 128(5):561-567.

[49] Destexhe, A. and Parè, D. (1999). Impact of network activity on the integrative properties of neocortical pyramidal neurons in vivo. J Neurophysiol, 81:1531-1547.

[50] Doyle, D., Cabral, J. M., Pfuetzner, R., Kuo, A., Gulbis, J., Cohen, S., Chait, B., and MacKinnon, R. (1998). The structure of the potassium channel: molecular basis of k+ conduction and selectivity. Science, 280(5360):69-77.

[51] Duflocq, A., Le Bras, B., Bullier, E., Couraud, F., and Davenne, M. (2008). Nav1.1 is predominantly expressed in nodes of ranvier and axon initial segments. Mol Cell Neurosci, 39(2):180-192.

[52] Dzhashiashvili, Y., Zhang, Y., Galinska, J., Lam, I., Grumet, M., and Salzer, J. (2007). Nodes of ranvier and axon initial segments are ankyrin-g-dependent domans that assemble by distinct mechanisms. J Cell Biol, 177:857-870.

[53] Eccles, J. C., Libet, B., and Young, R. R. (1958). The behavior of chromatolysed motoneurons studied by intracellular recording. J Physiol, 143:11-40.

[54] Edwards, C. and Ottoson, D. (1958). The site of impulse initiation in a nerve cell of a crustacean stretch receptor. J Physiol, 143:138-148. 
[55] Engel, D. and Jonas, P. (2005). Presynaptic action potential amplification by voltage-gated na ${ }^{+}$channels in hippocampal mossy fiber boutons. Neuron, 143:405417.

[56] Ermentrout, B., Pascal, M., and Gutkin, B. (2001). The effects of spike frequency adaptation and negative feedback on the synchronization of neural oscillators. Neural Comput, 13:1285-1310.

[57] Farinas, I. and DeFelipe, J. (1991). Patterns of synaptic input on corticocortical and corticothalamic cells in the cat visual cortex. ii. the axon initial segment. $J$ Comp Neurol, 304(1):70-77.

[58] Fatt, P. (1957). Na+ imaging reveals little difference in action potential-evoked nat influx between axon and soma. J Neurophysiol, 20:61-80.

[59] Fleidervish, I. and Gutnick, M. (1996). Kinetics of slow inactivation of persistent sodium current in layer $\mathrm{v}$ neurons of mouse neocortical slices. J Neurophysiol, 76(3):2125-2130.

[60] Fleidervish, I., Lasser-Ross, N., Gutnick, M., and Ross, W. (2010). Na+ imaging reveals little difference in action potential-evoked na ${ }^{+}$influx between axon and soma. Nat Neurosci, 13(7):852-860.

[61] Fourcaud, N. and Brunel, N. (2002). Dynamics of the firing probability of noisy integrate-and-fire neurons. Neural Comput, 14(9):2057-2110.

[62] Fourcaud-Trocme, N. and Brunel, N. (2005). Dynamics of the instantaneous firing rate in response to changes in input statistics. J Comput Neurosci, 18(3):311321.

[63] Fourcaud-Trocme, N., Hansel, D., van Vreeswijk, C., and Brunel, N. (2003). How spike generation mechanisms determine the neuronal response to fluctuating inputs. J Neurosci, 23 (37):11628-11640.

[64] Franzini-Armstrong, C. and Protasi, F. (1997). Ryanodine receptors of striated muscles: a complex channel capable of multiple interactions. Physiol Rev, $77(3): 699-729$.

[65] Gasparini, S., Migliore, M., and Magee, J. (2004). On the initiation and propagation of dendritic spikes in ca1 pyramidal neurons. J Neurosci, 24(49):11046-11056.

[66] Gerstner, W. (2000). Population dynamics of spiking neurons: fast transients, asynchronous states, and locking. Neural Comput, 12(1):43-89. 
[67] Golding, N., Kath, W., and Spruston, N. (2001). Dichotomy of action-potential backpropagation in ca1 pyramidal neuron dendrites. J Neurophysiol, 86(6):29983010 .

[68] Golding, N. and Spruston, N. (1998). Dendritic sodium spikes are variable triggers of axonal action potentials in hippocampal ca1 pyramidal neurons. Neuron, 21(5):1189-1200.

[69] Green, D. and Swets, J. (1966). Signal detection theory and psychophysics. John Wiley, Oxford, England.

[70] Greenberg, D., Houweling, A., and Kerr, J. (2008). Population imaging of ongoing neuronal activity in the visual cortex of awake rats. Nat Neurosci, 11:749-751.

[71] Grubb, M. and Burrone, J. (2010a). Activity-dependent relocation of the axon initial segment fine-tunes neuronal excitability. Nature, 465(7301):1070-1074.

[72] Grubb, M. and Burrone, J. (2010b). Building and maintaining the axon initial segment. Curr Opin Neurobiol, 20(4):481-488.

[73] Gründemann, J. and Häusser, M. (2010). Neuroscience: A plastic axonal hotspot. Nature, 465(7301):1022-1023.

[74] Gutnick, M. and Crill, W. (1995). The cortical neuron as an electrophysiological unit. In MJ, G. and I, M., editors, The cortical neuron, pages 33-51. Oxford UP, New York.

[75] Häusser, M., Stuart, G., Racca, C., and Sakmann, B. (1995). Axonal initiation and active dendritic propagation of action potentials in substantia nigra neurons. Neuron, 15(3):637-647.

[76] Hedstrom, K., Ogawa, Y., and Rasband, M. (2008). Ankyrin g is required for maintenance of the axon initial segment and neuronal polarity. J Cell Biol, 187(4):635-640.

[77] Henze, D. and Buzsaki, G. (2001). Action potential threshold of hippocampal pyramidal cells in vivo is increased by recent spiking activity. Neuroscience, 105(1):121-130.

[78] Herrmann, A. and Gerstner, W. (2002). Noise and the psth response to current transients: Ii. integrate-and-fire model with slow recovery and application to motoneuron data. J Comput Neurosci, 12 (2):83-95.

[79] Herz, A., Gollisch, T., Machens, C., and Jaeger, D. (2006). Modeling singleneuron dynamics and computations: a balance of detail and abstraction. Science, 314(5796):80-85. 
[80] Higgs, M., Slee, S., and Spain, W. (2006). Diversity of gain modulation by noise in neocortical neurons: regulation by the slow afterhyperpolarization conductance. J Neurosci, 26(34):8787-8799.

[81] Hô, N. and Destexhe, A. (2000). Synaptic background activity enhances the responsiveness of neocortical pyramidal neurons. J Neurophysiol, 84 (3):1488-1496.

[82] Hodgkin, A. (1975). The optimum density of sodium channels in an unmyelinated nerve. Philos Trans R Soc Lond B Biol Sci., 270(908):297-300.

[83] Hodgkin, A. and Huxley, A. (1952). A quantitative description of membrane current and its application to conduction and excitation in nerve. J Physiol, 117:500544.

[84] Holt, G., Softky, W., Koch, C., and Douglas, R. (1996). Comparison of discharge variability in vitro and in vivo cat visual cortex neurons. J Neurophysiol, 75(5):1806-1814, YEAR =.

[85] Hopfield, J. (2003). Pattern recognition computation using action potential. J Neurosci, 23 (37):11628-11640.

[86] Hu, W., Tian, C., Li, T., Yang, M., Hou, H., and Shu, Y. (2009). Distinct contributions of na(v)1.6 and na(v)1.2 in action potential initiation and backpropagation. Nat Neurosci, 12(8):996-1002.

[87] Huang, H. (2006). Molecular mechanism of antimicrobial peptides: the origin of cooperativity. Biochim Biophys Acta, 1758(9):1292-1302.

[88] Huang, M. (2009). Spatio-temporal dynamics of pattern formation in the cerebral cortex : Visual maps, population response and action potential generation. $\mathrm{PhD}$ thesis, IMPRS in Neurosciences, Georg-August-Universität, Göttingen.

[89] Huang, M., Volgushev, M., and Wolf, F. (2011). Sodium channel cooperativity in action potential initiation and neural encoding. submitted.

[90] Inda, M., DeFelipe, J., and A, M. (2006). Voltage-gated ion channels in the axon initial segment of human cortical pyramidal cells and their relationship with chandelier cells. Proc Natl Acad USA, 103:2920-2925.

[91] Izhikevich (2004). Which model to use for cortical spiking neurons? IEEE Trans Neural Netw, 15(5):1063-1070.

[92] Johnston, J., Griffin, S., Baker, C., Skrzypiec, A., Chernova, T., and Forsythe, I. (2008). Initial segment kv2.2 channels mediate a slow delayed rectifier and maintain high frequency action potential firing in medial nucleus of the trapezoid body neurons. J Physiol, 586(14):3493-3509. 
[93] Jung, P. (1994). Threshold devices: Fractal noise and neural talk. Phys Rev E Stat Phys Plasmas Fluids Relat Interdiscip Topics, 50(4).

[94] Khaliq, Z. and Raman, I. (2006). Relative contributions of axonal and somatic na channels to action potential initiation in cerebellar purkinje neurons. J Neurosci, 26(7):1935-1944.

[95] Knight, B. (1972a). Dynamics of encoding in a population of neurons. J Gen Physiol, 59(6):734-766.

[96] Knight, B. (1972b). The relationship between the firing rate of a single neuron and the level of activity in a population of neurons. experimental evidence for resonant enhancement in the population response. J Gen Physiol, 59(6):767-778.

[97] Kole, M., Ilschner, S., Kampa, B., Williams, S., Ruben, P., and Stuart, G. (2008). Is action potential threshold lowest in the axon? Nat Neurosci, 11:178-186.

[98] Kole, M., Letzkus, J., and Stuart, G. (2007). Unknown title. Neuron, 55(4):633647.

[99] Kole, M. and Stuart, G. (2008). Action potential generation requires a high sodium channel density in the axon initial segment. Nat Neurosci, 11(11):12531255 .

[100] Köndgen, H., Geisler, C., Fusi, S., Wang, X., Lüscher, H., and Giugliano, M. (2008). The dynamical response properties of neocortical neurons to temproally modulated noisy inputs in vitro. Cereb Cortex, 18 (9):2086-2097.

[101] Kress, G., Dowling, M., Meeks, J., and Mennerick, S. (2008). High threshold, proximal initiation, and slow conduction velocity of action potentials in dentate granule neuron mossy fibers. J Neurophysiol, 100(1):281-291.

[102] Kuba, H., Ishii, T., and Ohmori, H. (2006). Axonal site of spike initiation enhances auditory coincidence detection. Nature, 444(7122):1069-1072.

[103] Kuba, H., Oichi, Y., and Ohmori, H. (2010). Presynaptic activity regulates na ${ }^{+}$ channel distribution at the axon initial segment. Nature, 465(7301):1075-1078.

[104] La Camera, G., Rauch, A., Thurbon, D., Lscher, H., Senn, W., and Fusi, S. (2006). Multiple time scales of temporal response in pyramidal and fast spiking cortical neurons. J Neurophysiol, 96(6):3448-3464.

[105] Larkman, A. (1991a). Dendritic morphology of pyramidal neurones of the visual cortex of the rat: I. branching patterns. J Comp Neurol, 306(2):307-319. 
[106] Larkman, A. (1991b). Dendritic morphology of pyramidal neurones of the visual cortex of the rat: Ii. parameter correlations. J Comp Neurol, 306(2):320-331.

[107] Larkman, A. (1991c). Dendritic morphology of pyramidal neurones of the visual cortex of the rat: Iii. spine distributions. J Comp Neurol, 306(2):332-343.

[108] Larkum, M., Zhu, J., and Sakmann, B. (2001). Dendritic mechanisms underlying the coupling of the dendritic with the axonal action potential initiation zone of adult rat layer 5 pyramidal neurons. J Physiol, 533(Pt 2):447-466.

[109] Lindner, B., Longtin, A., and Bulsara, A. (2003). Analytic expressions for rate and $\mathrm{cv}$ of a type i neuron driven by white gaussian noise. Neural Comput, 15(8):1760-1787.

[110] Lindner, B. and Schimansky-Geier, L. (2001). Transmission of noise coded versus additive signals through a neuronal ensemble. Phys Rev Lett, 86(14):29342937.

[111] Lipowsky, R., Gillessen, T., and Alzheimer, C. (1996). Dendritic na+ channels amplify epsps in hippocampal ca1 pyramidal cells. J Neurophysiol, 76:2181-2191.

[112] Lorincz, A. and Nusser, Z. (2008). Cell-type-dependent molecular composition of the axon initial segment. J Neurosci, 28(53):14329-14340.

[113] Losonczy, A. and Magee, J. (2006). Integrative properties of radial oblique dendrites in hippocampal ca1 pyramidal neurons. Neuron, 50(2):291-307.

[114] Machens, C., Schütze, H., Franz, A., Kolesnikova, O., Stemmler, M., Ronacher, B., and Herz, A. (2003). Single auditory neurons rapidly discriminate conspecific communication signals. Nat Neurosci, 6(4):341-342.

[115] Machens, C. and Zador, A. (2003). Auditory modeling gets and edge. J Neurophysiol, 90(6):3581-3582.

[116] Magistretti, J., Ragsdale, D., and Alonso, A. (1999). High conductance sustained single-channel activity responsible for the low-threshold persistent na+ current in entorhinal cortex neurons. J Neurosci, 19:7334-7341.

[117] Mainen, Z., Joerges, J., Huguenard, J., and Sejnowski, T. (1995). A model of spike initiation in neocortical pyramidal neurons. Neuron, 15(6):1427-1439.

[118] Martina, M. and Jonas, P. (1997). Functional differences in na ${ }^{+}$channel gating between fast-spiking interneurones and principal neurones of rat hippocampus. $J$ Physiol, 505(Pt 3):593-603. 
[119] Martina, M., Vida, I., and Jonas, P. (2000). Distal initiation and active propagation of action potentials in interneuron dendrites. Science, 287(5451):295-300.

[120] Marx, S., Ondrias, K., and Marks, A. (1998). Coupled gating between individual skeletal muscle $\mathrm{ca}^{2+}$ release channels (ryanodine receptors). Science, 281(5378):811-821.

[121] Matsumoto, E. and Rosenbluth, J. (1985). Plasma membrane structure at the axon hillock, initial segment and cell body of frog dorsal root ganglion cells. $J$ Neurocytol, 14(5):731-747.

[122] McCormick, D., Connors, B., Lighthall, J., and Prince, D. (1985). Comparative electrophysiology of pyramidal and sparsely spiny stellate neurons of the neocortex. J Neurophysiol, 54(4):782-806.

[123] McCormick, D., Shu, Y., and Yu, Y. (2007). Neurophysiology: Hodgkin and huxley model - still standing? Nature, 445:1-2.

[124] McCulloch, W. S. and Pitts, W. (1943). A logical calculus of the ideas immanent in nervous activity. Bull Math Biophysics, 5:115-133.

[125] Meeks, J. and Mennerick, S. (2007). Action potential initiation and propagationin ca3 pyramidal axons. J Neurophysiol, 97:3460-3472.

[126] Mittmann, T., Linton, S., Schwindt, P., and Crill, W. (1997). Evidence for persistent $\mathrm{na}^{+}$current in apical dendrites of rat neocortical neurons from imaging of na ${ }^{+}$-sensitive dye. J Neurophysiol, 78:1188-1192.

[127] Molina, M., Barrera, F., Fernàndez, A., Poveda, J., Renart, M., Encinar, J., Riquelme, G., and Gonzàlez-Ros, J. (2011). Clustering and coupled gating modulate the activity in kcsa, a potassium channel model. J Biol Chem, 281(27):1883718848.

[128] Moreno-Bote, R. and Parga, N. (2006). Auto- and crosscorrelograms for the spike response of leaky integrate-and-fire neurons with slow synapses. Phys Rev Lett, 96(2):028101.

[129] Naundorf, B. (2005). Dynamics of population coding in the cortex. PhD thesis, Mathematical Natural Sciences, Georg-August-Universität, Göttingen.

[130] Naundorf, B., Geisel, T., and Wolf, F. (2005a). Action potential onset dynamics and the response speed of neuronal populations. J Comput Neurosci, 18:297-309.

[131] Naundorf, B., Geisel, T., and Wolf, F. (2005b). Dynamical response properties of a canonical model for type-i membranes. Neurocomputing, 20:421-428. 
[132] Naundorf, B., Wolf, F., and Volgushev, M. (2006). Unique features of action potential initiation in cortical neurons. Nature, 440(7087):1060-1063.

[133] Naundorf, B., Wolf, F., and Volgushev, M. (2007). reply to: hodgkin-huxley model - still standing? Nature, 445:E2-E3.

[134] Neumcke, B. and Stämpfli, R. (1983). Alteration of the conductance of na ${ }^{+}$ channels in the nodal membrane of frog nerve by holding potential and tetrodotoxin. Biochim Biophys Acta, 727(1):177-184.

[135] Niven, J. and Laughlin, S. (2008). Energy limitation as a selective pressure on the evolution of sensory systems. J Exp Biol., 211(Pt 11)::1792-1804.

[136] Ogawa, Y. and Rasband, M. (2008). The functional organization and assembly of the axon initial segment. Curr Opin Neurobiol., 18(3):307-301.

[137] Ogiwara, I., Miyamoto, H., Morita, N., Atapour, N., Mazaki, E., Inoue, I., Takeuchi, T., Itohara, S., Yanagawa, Y., Obata, K., Furuichi, T., Hensch, T., and Yamakawa, K. (2007). Na(v)1.1 localizes to axons of parvalbumin-positive inhibitory interneurons: a circuit basis for epileptic seizures in mice carrying an scn1a gene mutation. J Neurosci, 27(22):5903-5914.

[138] Palay, S., Sotelo, C., Peters, A., and Orkand, P. (1968). The axon hillock and the initial segment. J Cell Biol, 38:193-201.

[139] Palmer, L. and Stuart, G. (2006). Site of action potential initiation in layer 5 pyramidal neurons. J Neurosci, 26:1854-1863.

[140] Pan, Z., Kao, T., Horvath, Z., Lemos, J., Sul, J., Cranstoun, S., Bennett, V., Scherer, S., and Cooper, E. (2006). A common ankyrin-g-based mechanism retains kcnq and nav channels at electrically active domains of the axon. J Neurosci, 26(10):2599-2613.

[141] Platkiewicz, J. and Brette, R. (2010). A threshold equation for action potential initiation. PLoS Comput Biol, 6(7).

[142] Post, J., Leunissen-Bijvelt, J., Ruigrok, T., and Verkleij, A. (2011). Ultrastructural changes of sarcolemma and mitochondria in the isolated rabbit heart during ischemia and reperfusion. Biochim Biophys Acta, 845(1):119-123.

[143] Ragsdale, D. (2008). How do mutant nav1.1 sodium channels cause epilepsy? Brain Res Rev, 58(1):149-159.

[144] Rall, W. (1959). Branching dendritic trees and motoneuron membrane resistivity. Exp Neurol, 1:491-527. 
[145] Rall, W. (1964). Theoretical significance of dendritic trees for neuronal inputoutput relations. Stanford Univ Press.

[146] Rall, W. (1977). Core conductor theory and cable properties in neurons, pages 39-97. Handbook of physiology. American Physiology Society, Bethesda, MD.

[147] Rall, W. (1989). Cable theory for dendritic neurons, pages 9-62. MIT Press.

[148] Raman, I. and Bean, B. (1997). Resurgent sodium current and action potential formation in dissociated cerebellar purkinje neurons. J Neurosci, 17(12):4517-4526.

[149] Rauch, A., LaCamera, G., Lüscher, H., Senn, W., and Fusi, S. (2003). Neocortical pyramidal cells respond as integrate-and-fire neurons to in vivo-like input currents. J Neurophysiol, 90:1598-1612.

[150] Richardson, M. (2004). Effects of synaptic conductance on the voltage distribution and firing rate of spiking neurons. Phys Rev E Stat Nonlin Soft Matter Phys, 69(5 Pt 1):051918.

[151] Richardson, M., Brunel, N., and Hakim, V. (2003). From subthreshold to firingrate resonance. J Neurophysiol, 89(5):2538-2554.

[152] Rieke, F., Warland, D., de Ruyter van Steveninck, R., and Bialek, W. (1999). Spikes: Exploring the neural code. MIT Press, Cambridge,England.

[153] Rinzel, J. and Ermentrout, G. B. (1989). Analysis of neuronal excitability and oscillations. MIT Press, Cambridge, MA.

[154] Rush, A., Dib-Hajj, S., and Waxman, S. (2005). Electrophysiological properties of two axonal sodium channels, nav1.2 and nav1.6, expressed in mouse spinal sensory neurones. J Physiol, 564(Pt 3):803-815.

[155] Saito, A., Inui, M., Radermacher, M., Frank, J., and Fleischer, S. (1988). Ultrastructure of the calcium release channel of sarcoplasmic reticulum. J Cell Biol, 107(1):211-219.

[156] Sarmiere, P., Weigle, C., and Tamkun, M. (2008). The kv2.1 k ${ }^{+}$channel targets to the axon initial segment of hippocampal and cortical neurons in culture and in situ. BMC Neurosci, 9:112.

[157] Schafer, D., Jha, S., Liu, F., Akella, T., McCullogh, L., and Rasband, M. (2009). Disruption of axon initial segment cytoskeleton is a new mechanism for neuronal injury. J Neurosci, 29(42):13242-13254.

[158] Schiller, J., Schiller, Y., Stuart, G., and Sakmann, B. (1997). Calcium action potentials restricted to distal apical dendrites of rat neocortical pyramidal neurons. J Physiol, 505 ( Pt 3):605-616. 
[159] Schmidt-Hieber, C. and Bischofberger, J. (2010). Fast sodium channel gating supports localized and efficient axonal action potential initiation. J Neurosci, 30(30):10233-42.

[160] Schultz, C., Koenig, H.-G., Turco, D. D., Politi, C., Eckert, G. P., Ghebremedhin, E., Prehn, J. H., Koegel, D., and Deller, T. (2006). Coincident enrichment of phosphorylated $\mathrm{i} \kappa \beta \alpha$, activated ikk, and phosphorylated p65 in the axon initial segment of neurons. Mol Cell Neurosci, 33:68-80.

[161] Sengupta, B., Stemmler, M., Laughlin, S., and Niven, J. (2010). Action potential energy efficiency varies among neuron types in vertebrates and invertebrates. PLoS Comput Biol, 6:e1000840.

[162] Shadlen, M. and Newsome, W. (1994). Noise, neural codes and cortical organization. Curr Opin Neurobiol, 4(4):569-579.

[163] Shah, M., Migliore, M., Valencia, I., Cooper, E., and Brown, D. (2008). Functional significance of axonal kv7 channels in hippocampal pyramidal neurons. Proc Natl Acad Sci U S A, 105(22):7869-7874.

[164] Shu, Y., Duque, A., Yu, Y., Haider, B., and McCormick, D. (2007). Properties of action-potential initiation in neocortical pyramidal cells: evidence from whole cell axon recordings. J Neurophysiol, 97(1):746-760.

[165] Shu, Y., Hasenstaub, A., Duque, A., Yu, Y., and McCormick, D. (2006). Modulation of intracortical synaptic potentials by presynaptic somatic membrane potential. Nature, 441:761-765.

[166] Silberberg, G., Bethge, M., Markram, H., Pawelzik, K., and Tsodyks, M. (2004). Dynamics of population rate codes in ensembles of neocortical neurons. J Neurophysiol, 91:704-709.

[167] Silberberg, S. and Magleby, K. (1997). Voltage-induced slow activation and deactivation of mechanosensitive channels in xenopus oocytes. J Physiol, 505(Pt 3):551-569.

[168] Softky, W. and Koch, C. (1993). The highly irregular firing of cortical cells is inconsistent with temporal integration of random epsps. J Neurosci, 13(1):334-350.

[169] Srinivasan, J., Schachner, M., and Catterall, W. (1998). Interaction of voltagegated sodium channels with the extracellular matrix molecules tenascin-c and tenascin-r. Proc Natl Acad Sci USA, 95(26):15753-15757.

[170] Stemmler, M. and Koch, C. (1999). How voltage-dependent conductances can adapt to maximize the information encoded by neuronal firing rate. Nat Neurosci, $2(6): 521-527$. 
[171] Stevens, C. and Zador, A. (1998). Input synchrony and the irregular firing of cortical neurons. Nat Neurosci, 1(3):210-217.

[172] Stuart, G. and Sakmann, B. (1994). Active propagation of somatic action potential into neocortical pyramidal cell dendrites. Nature, 367:69-72.

[173] Stuart, G., Schiller, J., and Sakmann, B. (1997). Action potential initiation and propagation in rat neocortical pyramidal neurons. J Physiol, 505:617-632.

[174] Svirskis, G. (2003). Fast response to small signals with noise in neurons. Nonlinear Analysis: Modelling and Control, 8(1):77-84.

[175] Svirskis, G. and Rinzel, J. (2000). Influence of temporal correlation of synaptic input on the rate and variability of firing in neurons. Biophys $J, 79(2): 629-637$.

[176] Tchumatchenko, T., Malyshev, A., Geisel, T., Volgushev, M., and Wolf, F. (2011). Correlations and synchrony in threshold neuron models. Phys Rev Lett, 104(5):058102.

[177] Thorpe, S., Fize, D., and Marlot, C. (1996). Speed of processing in the human visual system. Nature, 381:520-522.

[178] Tuckwell, H. C. (1988). Introduction to theoretical neurobiology, volume I - II. Cambridge University Press, Cambridge,England.

[179] Undrovinas, A., Fleidervish, I., and Makielski, J. (1992). Inward sodium current at resting potentials in single cardiac myocytes induced by the ischemic metabolite lysophosphatidylcholine. Circ Res, 71(5):1231-1241.

[180] Ursell, T., Huang, K., Peterson, E., and Phillips, R. (2007). Cooperative gating and spatial organization of membrane proteins through elastic interactions. PLoS Comput Biol, 3(5):e81.

[181] Van Wart, A., Trimmer, J., and Matthews, G. (2007). Polarized distribution of ion channels within microdomains of the axon initial segment. J Comp Neurol, 500(2):339-352.

[182] Volgushev, M., Malyshev, A., Balaban, P., Chistiakova, M., Volgushev, S., and Wolf, F. (2008). Onset dynamics of action potentials in rat neocortical neurons and identified snail neurons : quantification of the difference. PLoS ONE, 3:e1962.

[183] Wang, X. and Buzsàki, G. (1996). Gamma oscillation by synaptic inhibition in a hippocampal interneuronal network model. J Neurosci, 16:6402-6413.

[184] Wei, W. (2010). Theoretical studies of dynamics of action potential initiation and its role in neuronal encoding. PhD thesis, Georg-August-Universität, Göttingen. submitted. 
[185] Wei, W. and Wolf, F. (2011). Spike onset dynamics and response speed in neuronal populations. Phys Rev Lett, 106(8):088102.

[186] Wiesenfeld, K. and Moss, F. (1995). Stochastic resonance and the benefits of noise: from ice ages to crayfish and squids. Nature, 73(6509):33-36.

[187] Yu, Y., Shu, Y., and McCormick, D. (2008). Cortical action potential backpropogation explains spike threshold variability and rapid-onset kinetics. J Neurosci, 28:7260-7272.

[188] Yue, C., Remy, S., Su, H., Beck, H., and Yaari, Y. (2005). Proximal persistent na+ channels drive spike afterdepolarizations and associated bursting in adult ca1 pyramidal cells. J Neurosci, 25:9704-9720. 
168 


\section{Part V}

\section{Appendix}





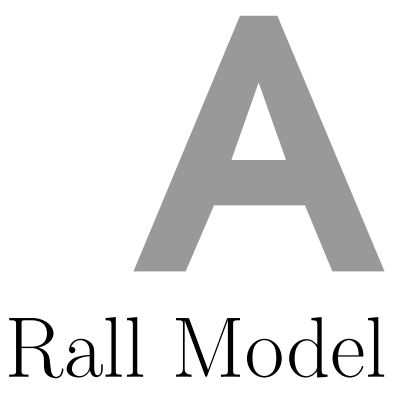

Thin extensions of neuron, such as axons and dendrites, are often idealized as cylinders. The cytoplasm and the extracellular fluid are both ionic media that conduct electric currents. Therefore, the neuronal extensions are also called as "core conductors (Rall [147]). More importantly, for distances shorter than the electrotonic length constant, the resistance across the surface is much higher than the resistance along the (both intracellular and extracellular) surface. This leads the current to flow in parallel to the cylinder axis for a considerable distance $(\approx \lambda)$ before a large fraction of the current is lost due to the leakage across the membrane.

For these reasons, the cable theory is commonly used for the mathematical representation of the neuronal extensions, i.e. when only a one-dimensional problem is confronted.

Gradient of the intracellular potential can be expressed as

$$
\begin{aligned}
\frac{\partial V_{i}}{\partial x} & =-i_{i} r_{i} \\
\frac{\partial^{2} V_{i}}{\partial x^{2}} & =-r_{i} \frac{\partial i_{i}}{\partial x}
\end{aligned}
$$

where $i_{i}$ is the intracellular current and $r_{i}$ is the intracellular resistance. One important assumption here is that $r_{i}$ does not change for a value of $x$, or in other words, the cable is uniform. Otherwise, the equation A.1 should include the term $-i_{i} \frac{\partial r_{i}}{\partial x}$ as well, which implies that the usual cable equation is not valid anymore. This assumption also points to the necessity of defining and analyzing the boundary conditions of the cable equation. These conditions should be stated to provide for the continuity of current and voltage, i.e. in the case of branching.

Until here, only $V_{i}$ was considered, but it can also be replaced with $V=V_{i}-V_{e}-E_{r}$, where $V_{e}$ is the external potential and $E_{r}$ is the membrane battery, while neither $V_{e}$ nor $E_{r}$ is dependent on $x$ or $t$. In addition to this, the right hand side of the equation is equal to the current that flows through the cable. So, equation A.1 becomes

$$
\frac{1}{r_{i}} \frac{\partial^{2} V}{\partial x^{2}}=i_{m}
$$


The passive neuronal membrane has a unit capacitance $c_{m}(\mathrm{~F} / \mathrm{cm})$, a unit conductance $g_{m}(\mathrm{~S} / \mathrm{cm})$ and a unit resistance $r_{m}(\Omega . \mathrm{cm})$. A simple equivalent circuit can represent the membrane potential dynamics for a unit distance $\Delta x$, such that

$$
\begin{aligned}
i_{m} & =c_{m} \frac{\partial V}{\partial t}+\frac{V_{i}-E_{r}-V_{e}}{r_{m}}, \\
r_{m} i_{m} & =\tau_{m} \frac{\partial V}{\partial t}+V
\end{aligned}
$$

\section{A.1 Boundary Conditions}

Some useful boundary conditions are (Rall [147])

\section{Finite cable (sealed-end) boundary condition:}

$$
\left.\frac{\partial V(x, t)}{\partial x}\right|_{x=x_{e n d}}=0
$$

where $x_{e n d}$ is one of the end points of the cable. Usually the starting point $x_{0}$ is taken as 0 .

2. Voltage-clamp boundary condition: At a certain point $x_{i}$, the voltage $V(t)$ $=V_{c}$. For instance, $V(t)=0 \Rightarrow V_{i}-V_{e}=E_{r}$ (the potential difference across the membrane is clamped to its resting value); $V(t)=-E_{r} \Rightarrow V_{i}=V_{e}$ (there is no voltage difference between extracellular and intracellular surface).

\section{Leaky end boundary condition:}

$$
\pm\left(\frac{\partial V(x, t)}{\partial x}\right) / r_{i}=V(x, t) G_{L} .
$$

4. Current injection at $x_{e n d}$ :

$$
\pm\left(\frac{\partial V(x, t)}{\partial x}\right) / r_{i}=I(t) .
$$

where $I(t)$ is the injected current. 
5. Branching at $x_{i}$ : For $n$ cables that are connecting, $n-1$ boundary conditions should be applied to provide for the continuity of $V$ and one boundary condition for conservation of $I$. These boundary conditions can be defined as above or a more complex boundary condition can be stated, e.g. an injected current at a leaky end.

6. Infinite end boundary condition: $V(t)$ remains bounded as $x \longrightarrow+\infty$ and $x \longrightarrow-\infty$.

7. Semi-infinite end boundary condition: $V(t)$ remains bounded as $x \longrightarrow$ $+\infty$ or $x \longrightarrow-\infty$.

A steady-state solution for the semi-infinite end boundary condition can be obtained by the following assumptions:

- $x_{0}=0$ and $x_{f}=+\infty$;

- Voltage-clamp at $x_{0}=0$, such that $V(t)=V_{0}$;

- Cable is uniform;

- There is no current injection.

The general solution for equation A.1 can be formulated as

$$
V(x)=A_{1} e^{\frac{x}{\lambda}}+A_{2} e^{-\frac{x}{\lambda}} .
$$

For $x \longrightarrow x_{f} \Rightarrow A_{1}=0$; and at $x_{0}=0 \Rightarrow A_{2}=V_{0}$. Therefore,

$$
V(x)=V_{0} e^{-\frac{x}{\lambda}} .
$$

This implies that the voltage decrements to $1 / e$ after a distance of $\lambda(V(x, t) / V(x)=$ $1 / e)$. When a termination point $x_{f}$ is more than four times $\lambda$ distant from an initial point $x_{0}$, there is negligible difference from semi-infinite cable. Therefore, when a neuronal extension is idealized as a cylindrical cable, it should have a length greater than $4 \lambda$. The Ball-and-Stick model that was described in chapter 6 satisfies this condition. 
174 Appendix A: Rall Model 


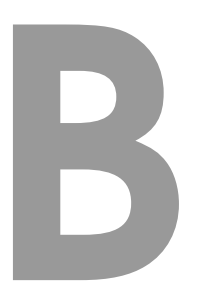

\section{Curriculum Vitae}

\section{PINAR ÖZ}

Theoretical Neurophysics Group

Max Planck Institute for Dynamics and Self-Organization

Georg-August-Universität Göttingen

Bunsenstrasse 10 Haus 2-205

37075 Gttingen

Date and place of birth: 08 August 1983

Ankara, Turkey

Citizenship: Turkish

E-mail: $\quad$ poz@nld.ds.mpg.de

Tel.: $\quad$ +49(0) 5515176421

\section{EDUCATION}

2006 Oct - 2011 Mar PhD Student in International Max Planck Research School in Neurosciences

Göttingen

Germany

2002 Sep - 2006 Jun BSc in Department of Molecular Biology and Genetics Bogazici University

Istanbul, Turkey

2001 Sep - 2002 Jun English Preparation Class in School of Foreign Languages Bogazici University

Istanbul, Turkey

\section{AWARDS}

- 2007/2008 Georg-Christoph-Lichtenberg scholarship. 


\section{RESEARCH INTERESTS}

Action potential initiation

Single neuron modeling

Ion Channel Gating

Spike Statistics

\section{LIST OF PUBLICATIONS}

\section{Poster Presentations}

- Oz P and Wolf F (2011) A model of cooperative $\mathrm{Na}^{+}$channel gating in Axon Initial Segment.

Neurizons, Goettingen

- Oz P and Wolf F (2011) Spike initiation and response dynamics of neuronal models with cooperatively gating $\mathrm{Na}^{+}$channels.

9th Goettingen Meeting of the German Neuroscience Society

- Oz P, Huang M and Wolf F. (2009) Spike initiation and linear response dynamics of neuronal models with cooperatively gating $\mathrm{Na}^{+}$channels.

GGNB Science Day, Goettingen

- Oz P, Wei W and Wolf F (2009) The effect of threshold variability on somatic spike statistics in multi-compartmental models.

Neurizons, Goettingen 